\title{
MISFITS: AN ETHNOGRAPHIC STUDY OF EXTREMELY FAT PATIENTS IN INTENSIVE CARE
}

\author{
By \\ CAROLINE HALES
}

\begin{abstract}
A thesis
submitted to the Victoria University of Wellington in fulfilments of the requirements for the degree of Doctor of Philosophy in Nursing
\end{abstract}

Victoria University of Wellington 2015 


\section{ABSTRACT}

Critically ill fat patients pose considerable healthcare delivery and resource utilisation challenges which are often exacerbated by the patients' critical condition and types of interventional therapies used in the intensive care environment. Added to these difficulties of managing care is the social stigma that is attached to being fat. Intensive care staff not only have to attend to the specific needs of the critically ill body but also navigate, both personally and professionally, the social terrain of stigma when providing care to this patient population.

The purpose of this research was to explore the culture and influences within the intensive care setting in which doctors and nurses cared for fat patients. A focused ethnographic approach was adopted to elicit the specific knowledge and 'situated' experiences of caring for critically ill fat patients from the perspectives of intensive care staff. The setting for this study was an 18 bedded tertiary intensive care unit (ICU) in New Zealand. Participant observation of care practices and interviews with intensive care staff were undertaken over a four month period. This study adopted an insider perspective throughout the research process as the study site was also my place of work. The dual tensions of the nurse and researcher position are reflexively explored through the thesis.

Key findings from this research reveal how fat patients were considered to be 'misfits' in the ICU as a result of not fitting the physical, medical, and social norms of intensive care practices. Staff managed their private perceptions of fatness during care situations through the use of emotional labour, behavioural regions, and face-work. Through the construction and presentation of the professional and private 'face', staff were able to establish positive social experiences for fat patients.

This study has brought new understandings of fatness; often percieved as the last socially accepted form of discrimination. Conceptualising fat patients as 'misfits' in the intensive care setting, reveals the performances of staff in managing the social awkwardness of fat stigma. The implications of this for healthcare is the provision of 
clinical services that are fit for purpose and a reconceptualisation of how staff use emotional labour in order to deliver non-discriminatory care to socially stigmatised fat patients.

Key words: Fatness, obesity, intensive care, nursing, medicine, emotional labour, face-work, behavioural regions, focused ethnography. 


\section{ACKNOWLEDGEMENTS}

First and foremost, I would like to acknowledge my gratitude to the staff and patients involved in this study. Without their co-operation and participation, my project would not have been possible and for that I am forever grateful.

I would like to thank my many supervisors for their guidance, encouragement and support throughout the process of conducting the study and writing up the thesis. In particular, I am deeply indebted to my current supervisors, who have kept me motivated to the very end to complete this thesis. To Dr Kay DeVries: thank you for your wealth of knowledge and expertise which you so willing shared with me, and for your unfailing support, constant encouragement, accessibility, sense of humour, and belief in me. To Professor Maureen Coombs: I can not thank you enough for stepping into the secondary supervisory role five months out from submitting the thesis. Your thoughtful guidance, generous support, and encouragement over this time has been amazing. I would also like to acknowledge the support and encouragement given to me by my friends, colleagues, and managers within my clinical and academic workplaces. Your kind words of encouragement have been greatly appreciated.

Lastly, I would like to thank my family who have all done their part in ensuring family life continued during my frequent absences to study. In particular, my husband, Kevin, for the endless weekends looking after our children, Lukas and Caitlin, who have never known life without their mummy studying... And yes Lukas, mummy's big book is now finished. 


\section{TABLE OF CONTENTS}



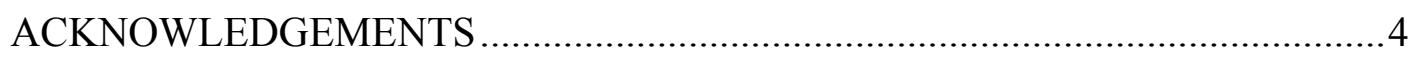

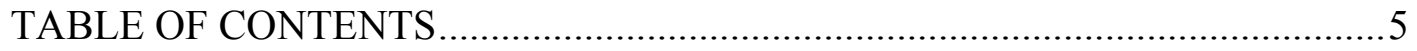



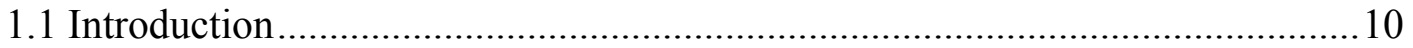







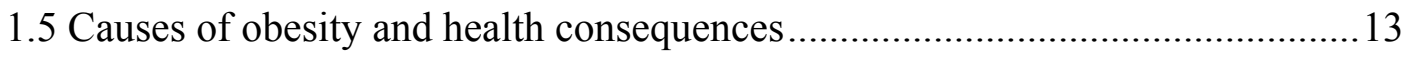

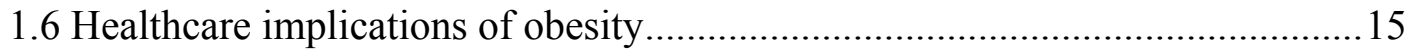

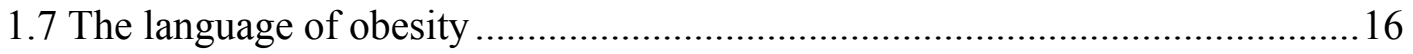

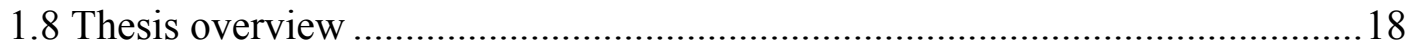

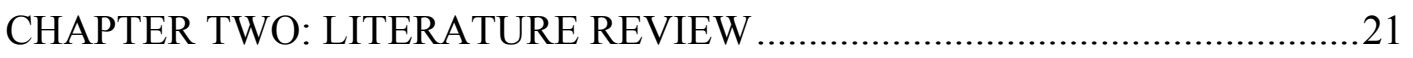

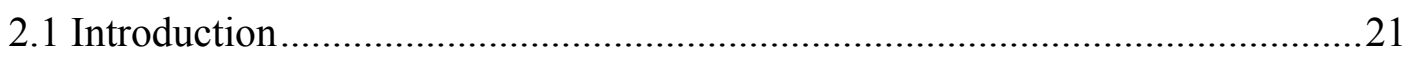

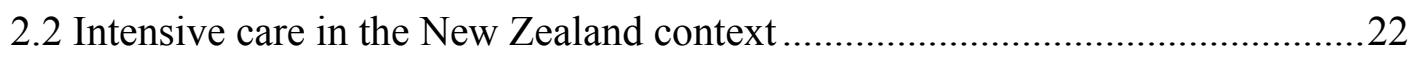

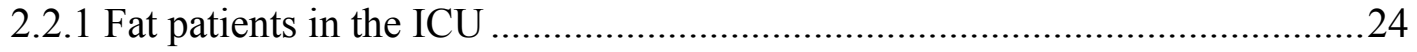

2.3 Healthcare professionals' perspectives of fatness...........................................26

2.3.1 Medical professionals' attitudes and beliefs about fatness ..........................22

2.3.2 Fat specialists' attitudes and beliefs about fatness.......................................28

2.3.3 Nurses' attitudes and beliefs about fatness .....................................................30

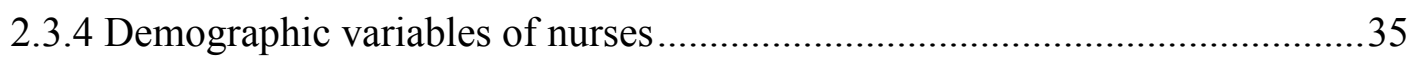

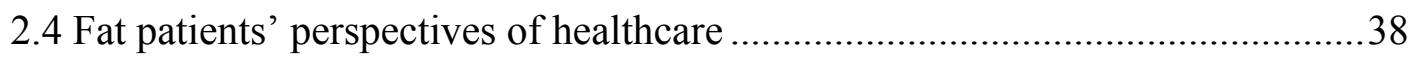

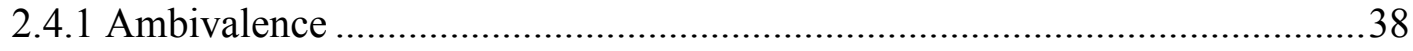

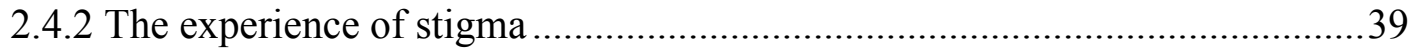

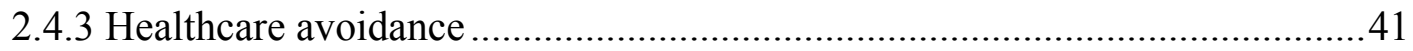

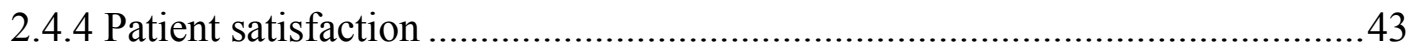

2.5 Theoretical perspectives of stigma, prejudice and discrimination....................43

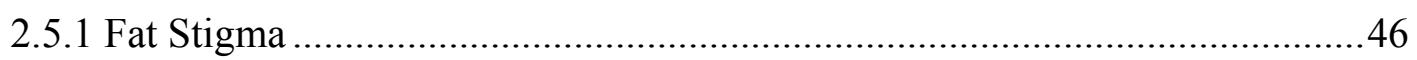

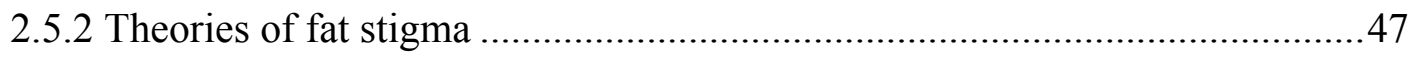

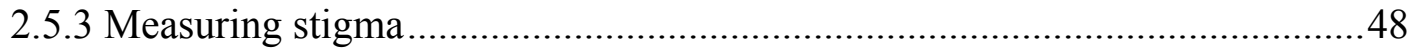

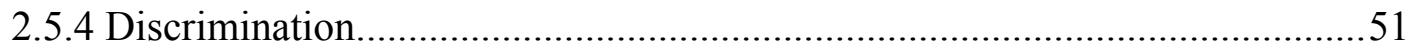


2.5.5 Stigma, prejudice and discrimination in healthcare .53

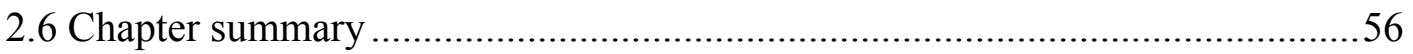

CHAPTER THREE: THEORETICAL INFLUENCES …....................................58

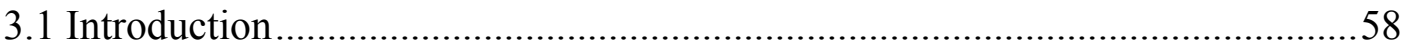

3.2 Goffman's dramaturgical perspective of self in social interaction ....................58

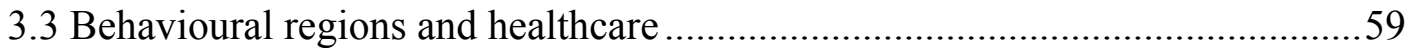

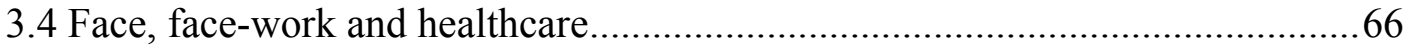

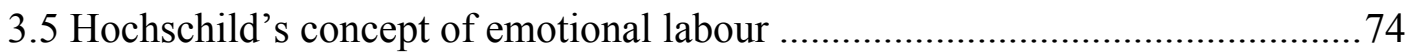

3.6 Emotional labour: An aspect of caring work .................................................. 77

3.7 Emotional labour: Interpersonal management of emotions ............................. 82

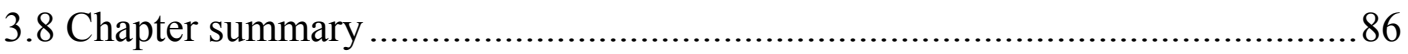

CHAPTER FOUR: METHODOLOGY AND METHODS …..............................8

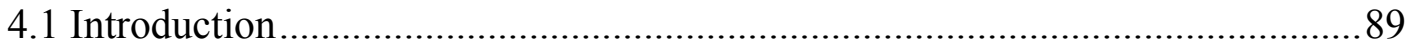

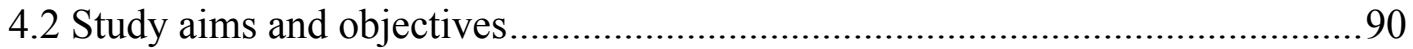

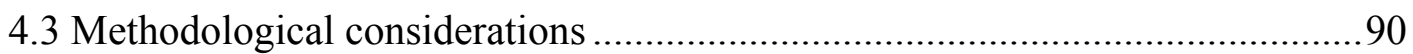

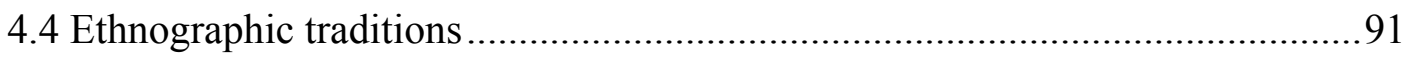



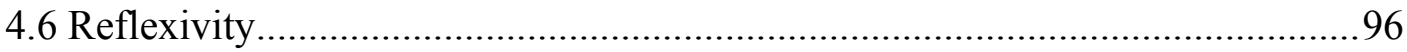

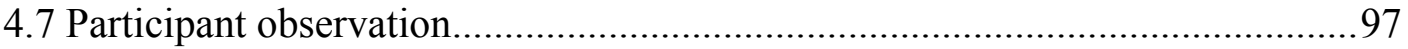

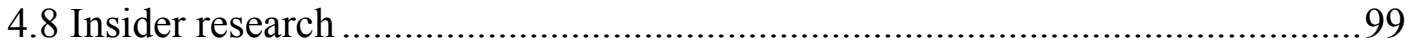

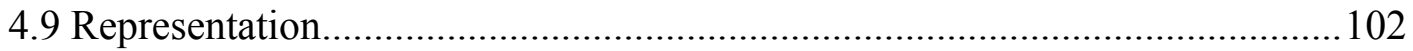

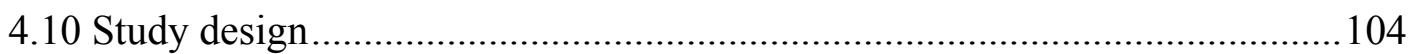

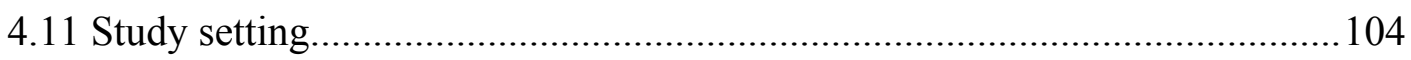

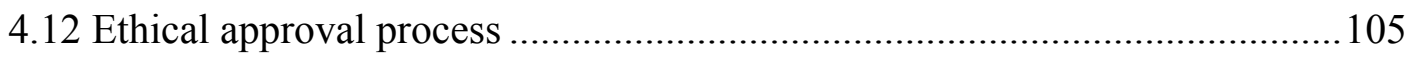

4.13 Participant selection, recruitment and sampling strategy ............................108



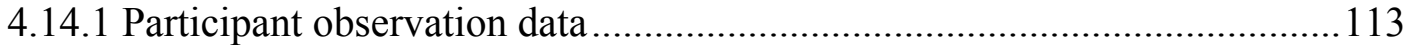

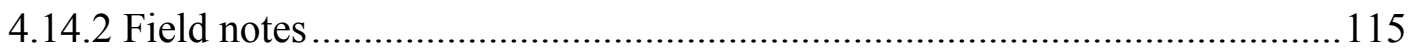

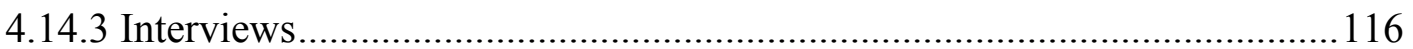

4.14.4 Cultural documents and material artefacts................................................... 119

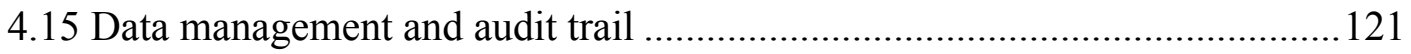

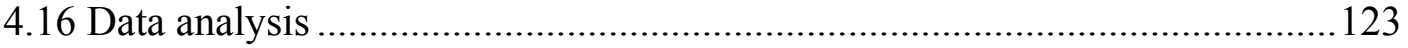

4.17 Protecting the identities of the study participants ......................................... 126 
4.18 Rigour and trustworthiness of the research process..................................... 128

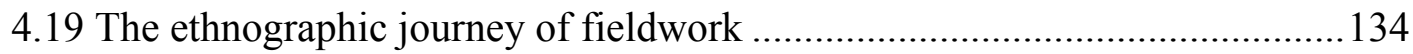

4.19.1 Entering the field and negotiating my researcher space ............................135

4.19.2 Leaving the field and re-negotiating my space as a nurse ........................139

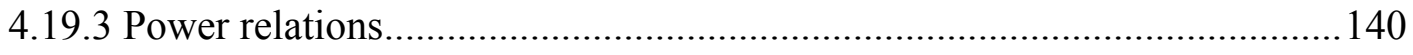

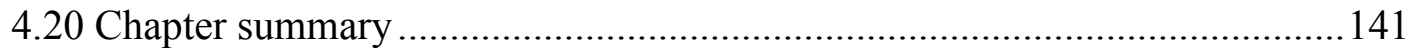

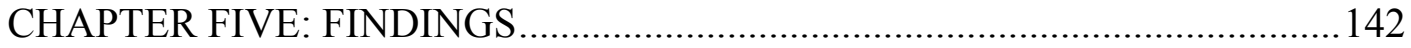

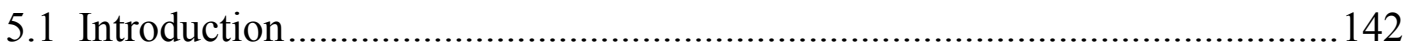

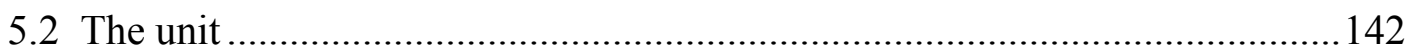

5.3 Conceptual overview of fat patients as 'Misfits' ........................................... 145

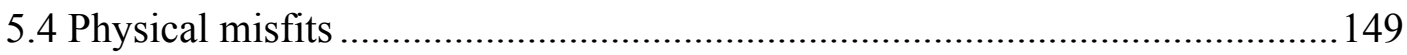

5.4.1 Consequences of being a physical misfit ................................................... 157

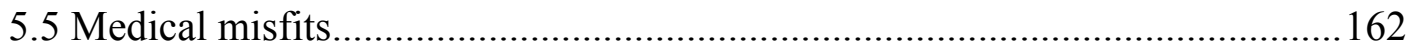

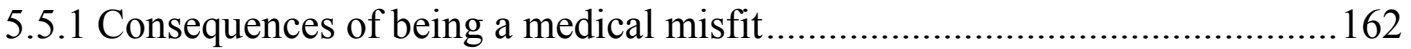



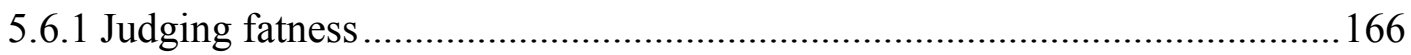

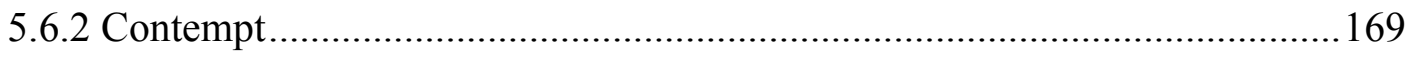

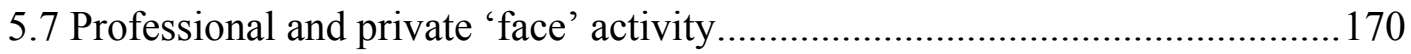

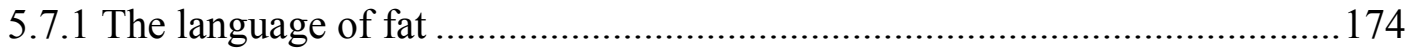

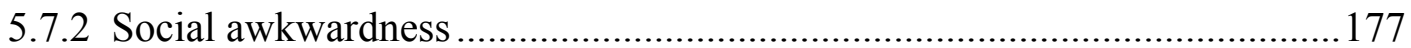

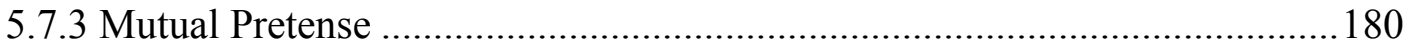

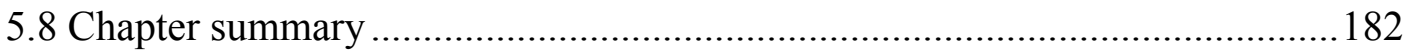

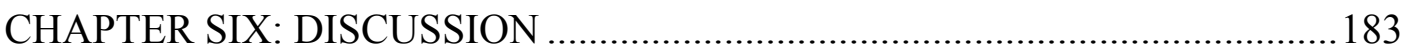

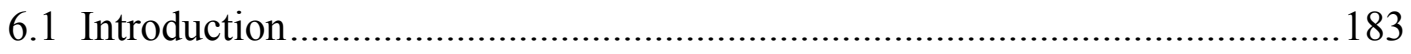

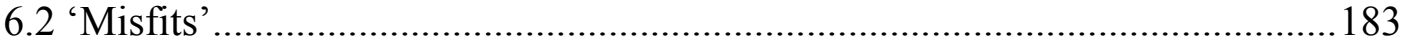

6.3 Managing 'misfits' through professional and private 'face' activity...............188

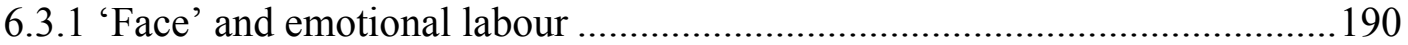

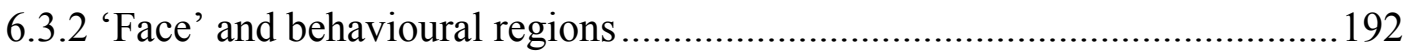

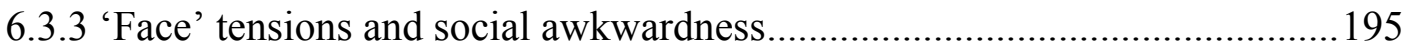

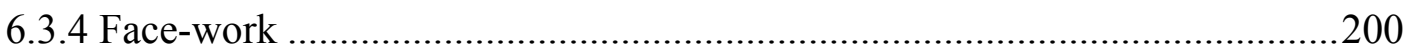



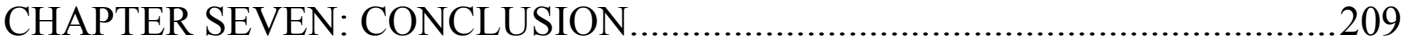

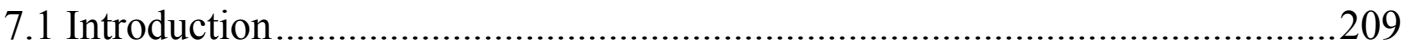






7.3 Contribution to methodology and method ..................................................212

7.4 Methodological critique of the study .........................................................213

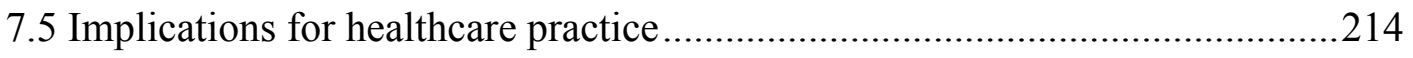

7.6 Recommendations for future research .....................................................216

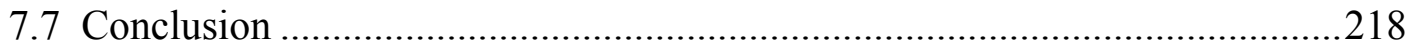

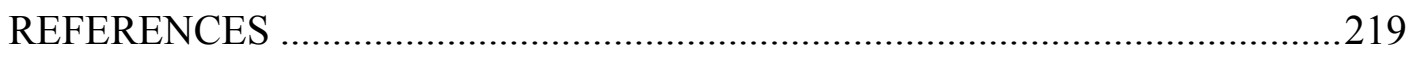

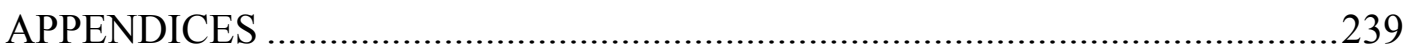

Appendix 1: Research Advisory Group for Māori consent .................................239

Appendix 2: National Ethics approval letter ....................................................241

Appendix 3: Amended National Ethics approval letter .......................................242

Appendix 4: Nurse study information sheet .......................................................243

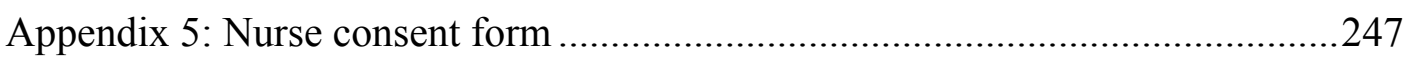

Appendix 6: Patient study information sheet....................................................249

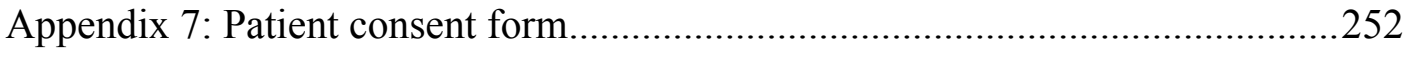



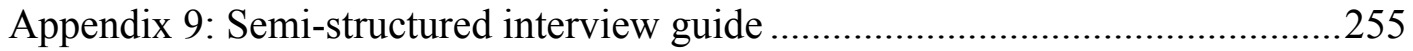

Appendix 10: Data management spreadsheet .......................................................256

Appendix 11: Data analysis: Example of operational definitions for raw data codes

Appendix 12: Data analysis: Example of raw data coding .................................259

Appendix 13: Data analysis/ conceptualisation ...............................................260 


\section{LIST OF TABLES}

Table 1. Classification of adults according to Body Mass Index (BMI).......12

Table 2. Current areas of research in the care of critically ill fat patients......24

Table 3. Data sets generated from the fieldwork study....................110

Table 4. Data labeling system within findings ...........................111

Table 5. Record of patient focused participant observation hours.............112

Table 6: Examples of annotated interview transcripts.....................114 


\section{CHAPTER ONE: INTRODUCTION}

\subsection{Introduction}

The care of critically ill morbidly obese patients within New Zealand healthcare settings pose considerable healthcare delivery and resource utilitisation challenges. Many of these challenges are excerbated by the patients' critical condition and types of interventional therapies used, that are specific to the intensive care environment. Added to these difficulties of managing care is the social stigma that is attached to being obese. Intensive care staff not only have to attend to the specific needs of the critically ill body but also navigate, both personally and professionally, the social terrain of stigma when providing care to this patient population. The impact of fat stigma on the nature and quality of social interactions between healthcare professionals and morbidly obese patients, in the context of care practices, is relatively unknown. Therefore, research into how morbidly obese patients are cared for within the specific setting of intensive care services is warranted.

\subsection{Research interest}

For many years I have had an interest in, and been clinically involved with, care issues for critically ill patients, particularly those people who are morbidly obese. This has been both as a practitioner and as an educator in the fields of critical care and bariatric $^{1}$ nursing. I have worked as a registered nurse for 15 years both in the United Kingdom and New Zealand. Most of my professional career has been working within the intensive care setting and more recently using my critical care skills to provide care for acutely deteriorating patients within the ward setting. I have worked in both public and private intensive care services within New Zealand. It was during my time working in the private setting that I became acutely aware of the differences in the quality of service provisions for morbidly obese patients. These starkly different experiences were instrumental in my selection of the research topic. In particular, my motives were influenced by the often upsetting life stories of morbidly obese patients

\footnotetext{
${ }^{1}$ The term bariatric refers to the practice of providing healthcare associated with the treatment of obesity and related conditions and can be used to refer to the actual size of the patient, the type of treatment, equipment or other resources necessary to provide care (Gallagher-Camden, 2006).
} 
who had resorted to bariatric surgery as a way of finally fitting in to the expectations that society had placed upon them to be thin.

My experiences of caring for morbidly obese patients concerned me both personally and professionally. From a personal perspective, I believe all individuals requiring healthcare should receive the same quality and standard of care at the point of accessing healthcare services; this did not appear to be the case. Instead the quality of care for each patient accessing the intensive care services within the same organisation appeared to be influenced by the availability of appropriate resources and occassionally societal attitudes. Professionally, my experiences suggested that as a nursing profession, we were not advocating effectively enough to ensure that care was fit for purpose when managing vunerable patient populations during a time of critical illness. These experiences, in conjunction with a review of the literature, led me to the assumptions that morbidly obese people were potentially marginalised and treated differently within healthcare. Hence, understanding how and why morbidly obese patients were cared for in particular ways became the focus of the research.

\subsection{Defining obesity}

Obesity is defined as abnormal or excessive body fat accumulation sufficiently large enough to adversely impair health and reduce longevity (Allison et al., 2008; Villareal, Apovian, Kushner, \& Klien, 2005; World Health Organisation, 2000). Impaired health may not always be immediately apparent in all individuals but obesity probabilistically threatens future health and life expectancy (Allison et al., 2008). The body mass index (BMI), a measure of weight adjusted for height, is the most commonly used indirect method to assess body fat (Ministry of Health, 2009; Sassi, 2010). The BMI categorises obesity into different classes and identifies the risks of co-morbidities associated with it (World Health Organisation, 2000). A BMI greater or equal to $30 \mathrm{~kg} / \mathrm{m}^{2}$ classifies someone as obese (see Table 1). 
Table 1. Classification of adults according to Body Mass Index (BMI)

\begin{tabular}{|l|l|l|l|}
\hline Classification & Class & BMI $\left(\mathrm{kg} / \mathrm{m}^{2}\right)$ & $\begin{array}{l}\text { Risk of co- } \\
\text { morbidities }\end{array}$ \\
\hline Normal weight & & $18.50-24.99$ & Average \\
\hline Overweight (pre-obese) & & $25.00-29.99$ & Increased \\
\hline Obese (mild) & I & $30.00-34.99$ & Moderate \\
Obese (moderate) & II & $35.00-39.99$ & Severe \\
Obese (Extreme, morbid, bariatric) & III & $40.00-44.99$ & Very severe \\
Obese (Super-morbid) & IV & $\geq 45.00$ & Very severe \\
& & & \\
\hline
\end{tabular}

(Adapted from WHO (2000) and Grieve, Fenwick, Yang, \& Lean (2013))

The BMI, as an indirect measure of obesity, has been highly criticised for its inability to differentiate between fat and lean mass, provide information on the distribution of body fat, or incorporate the variations in the ratio of fat to lean mass in different ethnicities (Ministry of Health, 2009; Prentice \& Jebb, 2001). For these reasons revised versions of the BMI classification parameters have been developed for individualised clinical use in Asian (World Health Organisation Expert Consultation, 2004), and Pacific Island populations (Duncan, Schofield, Duncan, Kolt, \& Rush, 2004). Similarly, waist circumstance, and waist:hip ratios are used to complement clinical data regarding fat distribution and health risk (Ministry of Health, 2009; World Health Organisation, 2000). Despite these limitations there is consistent evidence to support the association of increasing BMI with mortality and morbidity at population-level analysis (Grieve et al., 2013; Prospective Studies Collaboration, 2009).

\subsection{Obesity prevalence}

Obesity has become a common condition in many countries, including New Zealand, and a major concern for public health (Ministry of Health, 2004; Sassi, 2010). The World Health Organisation (2000) identified obesity as one of the most significant world-wide health problems of today, affecting three times more people than 20 years ago. Since the late 1980s the prevalence of New Zealand adults who are obese has increased from 10\% (Ministry of Health, 2004) to 31\% in 2013 (Ministry of Health, 2013). Obesity rates are significantly higher among New Zealanders who are 
living in socio-economically deprived areas and are of Māori ${ }^{2}$ or Pacific ethnicity (Ministry of Health, 2013). Approximately 48\% of Māori adults and 68\% of Pacific adults were obese in 2012 (Ministry of Health, 2013). Furthermore, at the same time that the prevalence of obesity has increased, so too has the extent to which people are morbidly obese.

Morbid obesity, a BMI greater or equal to $40 \mathrm{~kg} / \mathrm{m}^{2}$, is the fastest growing category of obesity in developed countries (Bromley \& Given, 2011; Grieve et al., 2013; Ministry of Health, 2004). Between 1977 and 2003, morbid obesity in New Zealand increased from $0.32 \%$ in males and $1.17 \%$ in females to $2.15 \%$ and $2.95 \%$, respectively, with the most rapid growth occurring after 1997 (Ministry of Health, 2004). This is consistent with other developed Organisation for Economic Cooperation Development (OECD) countries where morbid obesity prevalence has tripled over the last three decades and affects approximately $3 \%$ of the population (Bromley \& Given, 2011; Shields, Carroll, \& Ogden, 2011; Tjepkema, 2008).

Historically, patients with a BMI $\geq 40 \mathrm{~kg} / \mathrm{m}^{2}$ were considered to be one group, but with more frequently reported incidences of people with BMI's $\geq 65 \mathrm{~kg} / \mathrm{m}^{2}$ there has been a need to delineate this group further to evaluate health risks and care implications that may exist as BMI increases (Westerly \& Dabbagh, 2011). For this reason, classifications of obesity are regularly being expanded, and research regarding morbid and super-morbid obesity are of particular interest to healthcare.

\subsection{Causes of obesity and health consequences}

Excessive body fat occurs as a result of a positive energy imbalance caused by a chronic excess of energy intake over energy expenditure (Ministry of Health, 2004; World Health Organisation, 2000). The causative factors for this energy imbalance are multifactorial and not fully understood but have been strongly linked to genetical, environmental, societal and behavioural influences (Aronne, Nelinson, \& Lillo, 2009; Roth, Qiang, Marban, Redelt, \& Lowell, 2004; World Health Organisation, 2000). The amount and distribution of this fat within the body differs considerably between

\footnotetext{
${ }^{2}$ Indigenous population of New Zealand
} 
individuals and affects the risks associated with obesity and types of disease that result (Hirani, Zaninotto, \& Primatesta, 2008; World Health Organisation, 2000).

The health consequences of obesity are considerable and varied, ranging from premature death (Fontaine, Redden, Wang, Westfall, \& Allison, 2003) and increased mortality risks (Flegal, Kit, Orpana, \& Graubard, 2013), to debilitating disorders that have significant effects on an individual's health-related quality of life (Jia \& Lubetkin, 2005) and social participation (Forhan, Law, Vrkljan \& Taylor, 2010). The major health consequences are type 2 diabetes (Hirani et al., 2008; Kodama et al., 2014), obstructive sleep apnoea (Sánchez-de-la-torre et al., 2012; Soylu et al., 2012), hypertension (Hirani et al., 2008; Kotchen, 2010), coronary artery disease (Hubert, Feinleib, McNamara, \& Castelli, 1983; Siavash, Sadeghi, Salarifar, Amini, \& Shojaee-moradie, 2009), cardiomyopathy (Alpert, Fraley, Birchem, \& Senkottaiyan, 2005), osteoarthritis (Laberge et al., 2012; Pearson-Ceol, 2007), soft tissue infections (Huttunen \& Syrjänen, 2013; Karppelin et al., 2010), and psychosocial problems (Friedman et al., 2005; Karlsson, Taft, Sjöström, Torgerson, \& Sullivan, 2003; Wadden et al., 2006).

The mechanisms leading to psychosocial problems associated with obesity, such as depression, isolation, low self-esteem and body image distress are not primarily caused by the pathophysiological changes that occur within in the body but rather the culture-bound values in which society views fat bodies (Friedman et al., 2005; Karlsson et al., 2003; MacLean et al., 2009; World Health Organisation, 2000). For this reason, obesity is often referrred to as a 'social disease' because the psychological health consequences are related to social discrimination rather than biophysical dysfunction (Gard \& Wright, 2005). Thus, obesity has been recognised as a highly stigmatised condition in many developed countries in terms of the body's undesirable appearance and of the supposed personality traits possessed by that person (Puhl \& Brownell, 2003; World Health Organisation, 2000). 


\subsection{Healthcare implications of obesity}

The socio-economic burden of obesity is significant in terms of direct healthcare costs, loss of economic productivity ${ }^{3}$, and resource utilisation (Grieve et al., 2013; Sassi, 2010; Withrow \& Alter, 2011). Healthcare costs for treating obesity and related co-morbidities is estimated to account for between $1 \%$ and 3\% of a country's total healthcare expenditure (Sassi, 2010; Withrow \& Alter, 2011). This is with the exception of the United States where an estimated 5-10\% of total health expenses are related to obesity (Tsai, Williamson, \& Glick, 2011). Additionally, medical costs are approximately $30 \%$ higher than for non-obese individuals. In New Zealand, direct healthcare costs of treating obesity in 2004 was approximately \$460 million (Ministry of Health, 2008a) and loss of economic productivity, due to premature death associated with type 2 diabetes, ischaemic heart disease and stroke, was attributed to approximately 3,200 deaths per year between 1997 and 2001 (Ministry of Health \& University of Auckland, 2003).

In response to this socio-economic burden many governments within the OECD have attempted to address obesity through adopting initiatives aimed at promoting a culture of healthy eating and active living (Department of Health, 2011; Department of Health and Ageing, 2007; Ministry of Health, 2003; Sassi, 2010). 'Improving nutrition' and 'reducing obesity' were priority objectives in the New Zealand Health strategy between 2000 and 2009 (Ministry of Health, 2008b). Key initiatives aimed at increasing fruit and vegetable consumption and increasing the frequency of regular physical activity (Ministry of Health, 2003, 2008b). At the same time, clinical guidelines for weight management, specifically focused on improving weight outcomes for Māori, Pacific and South Asian ${ }^{4}$ populations, were developed and implemented throughout the country (Ministry of Health, 2009, 2010).

Only a small percentage of the overall New Zealand population are morbidly obese, however the significant health needs of this population group pose

\footnotetext{
${ }^{3}$ Loss of economic productivity relates to worker absenteeism, staff turnover, and reduced worker productivity as a result of obesity-related morbidity, together with lost earnings due to premature death from obesity-related disease (Grieve et al., 2013; World Health Organisation, 2000).

4 'South Asian'includes population groups originating from India (including Fijian Indians), Pakistan, Bangladesh, Sri Lanka, Nepal, Afghanistan, Bhutan, and the Maldives (Ministry of Health, 2009).
} 
considerable healthcare delivery and resource utilisation challenges (Westerly \& Dabbagh, 2011; Winkelman \& Maloney, 2005). As the BMI of the morbidly obese increases so too does the need for and duration of intensive care services (Villavicencio et al., 2007; Westerly \& Dabbagh, 2011). Morbidly obese patients admitted to the intensive care unit (ICU) are more likely to require prolonged mechanical ventilation and tracheostomy tube ${ }^{5}$ placement (Westerly \& Dabbagh, 2011; Yaegashi, Jean, Zuriqat, Noack, \& Homel, 2005), have increased length of ICU stay (Martino et al., 2011; Oliveros \& Villamor, 2008; Sakr et al., 2012; Villavicencio et al., 2007; Westerly \& Dabbagh, 2011; Winkelman \& Maloney, 2005; Yaegashi et al., 2005), increased respiratory and wound complications (Villavicencio et al., 2007; Winkelman \& Maloney, 2005; Yaegashi et al., 2005), and require significantly increased staffing support and specialist bariatric equipment (Winkelman \& Maloney, 2005). The need to manage the unique needs of morbidly and super-morbidly obese patients has driven the development of a sub-specialty of practice known as bariatric nursing (Gallagher-Camden, 2006).

Tertiary care facilitates play a key role in providing care to patients who are morbidly obese. However, there is a concern that the attitudes and beliefs of healthcare professionals regarding obesity may impact on the relationship between healthcare professionals and morbidly obese patients, and the quality of care that these patients receive (Merrill \& Grassley, 2008; Mold \& Forbes, 2013). Despite the evidence that indicates morbidly obese patients are viewed more negatively than normal weight patients by healthcare professionals (Schwartz, Chambliss, Brownell, Blair, \& Billington, 2003; Teachman \& Brownell, 2001), little is known about how doctors and nurses engage with morbidly obese patients during care provision within hospital settings.

\subsection{The language of obesity}

The terminology used to describe someone who has an excessive accumulation of body fat is a contentious issue resulting from the juxtaposed positions held by medicine/health and sociology as to whether excessive fat is a disease

\footnotetext{
${ }^{5}$ Tracheostomy tube is a breathing device inserted through the neck into the trachea to support prolonged mechanical ventilation in patients with respiratory failure (Bersten \& Soni, 2009).
} 
(Allison et al., 2008; Aronne et al., 2009; Kopelman \& Finer, 2001) or a natural way of being (Heshka \& Allison, 2001; Rothblum, 2012; Wann, 2009). These different perspectives, on the meaning and significance of body fat composition, has influenced the direction and focus of research, leading to two largely separate literatures and language. Despite these distinct languages, terminology is often used interchangeably within the layperson context.

The words 'overweight', 'obese', 'morbidly obese', and 'obesity' are predominantly used in biomedical obesity discourse to define a medical condition which is used to predict health risks and associated diseases (World Health Organisation, 2000). Research in this field focuses on understanding obesity as a disease, and developing preventative and management strategies at both individual and population-based levels (Ministry of Health, 2003, 2009). This is achieved through knowledge predominantly acquired from epidemiological, physiological and clinical studies. Thus, biomedical understandings of obesity are grounded in a language of disease, risk, prevention, and treatment.

In contrast, the word 'fat' is more commonly used in biopolitical obesity discourse within the field of fat studies. Since the late 1960s there has been a political agenda, by pro-fat acceptance movements, to reclaim the word 'fat' both as the preferred neutral adjective (Longhurst, 2005) and a preferred term of political identity (Wann, 2009). Through claiming and using the word 'fat' as a descriptor (not a discriminator) fat studies scholars deliberately position themselves apart from biomedical obesity discourse (Cooper, 2010; Wann, 2009). Fat studies scholarship critically examines societal attitudes regarding body weight and appearance from a perspective that like height, weight is a human characteristic (Rothblum, 2012). Research in this field challenges traditional understandings of obesity and uses "the language, culture and theory of civil rights, social justice and social change" (Cooper, 2010, p. 1020). Therefore, biopolitical understandings of obesity are embedded in a language of power, oppression, stigma, prejudice and discrimination.

For the purpose of this thesis I have chosen to use the word 'fat' to describe patients with excessive accumulation of body fat. The reason for this choice of 
language is a pragmatic one, primarily in recognition of, and respect for, the patient participants in this and other studies, who voiced their preference for the use of the word 'fat' over 'obese' (Wadden \& Didie, 2003). Therefore, the use of the word fat is not a decision to situate the thesis in the field of fat studies. This same approach to the terminology used to describe obesity has been adopted by other scholars working across sociology and health disciplines (Crandall, 1994; Pepper \& Ruiz, 2007; Thomas, Hyde, Karunaratne, Herbert, \& Komesaroff, 2008). Therefore, within the thesis the terms fat and fatness will be used to refer to obese, extremely obese, obesity and morbid obesity, unless it is contained within a direct quote.

\subsection{Thesis overview}

This thesis is presented in seven chapters. This first chapter presents an introduction to the thesis, and my interest and reasons for choosing to undertake this research. It has provided background to the study by presenting the significance of fatness in comtemporary society in terms of prevalence, and the socio-economic and healthcare implications of being fat. Additionally, it has drawn attention to the juxtaposed positions held by medicine/ health and sociology in regards to the medical and social context of fatness which led to the careful consideration of the language used within the thesis.

The purpose of Chapter Two is to present the current evidence and identify the gaps in knowledge of how critically ill fat patients are cared for within the intensive care setting. The chapter provides background into the intensive care setting and identifies the emerging areas of research into the care of this patient population. Theoretical perspectives that underpin stigma, prejudice and discrimination, as they apply to the healthcare setting and health literature, are examined to understand how the social stigma of fatness may influences intensive care practices.

Chapter Three introduces the works of Erving Goffman (1959, 1967, 1969) and Arlie Hochschild (1983) which have been instrumental in understanding the care practices and social interactions between staff and fat patients as they emerged from the analysis of the data. An exploration of their work in relation to social interaction 
and the management of emotions is presented followed by a critique of their application to the healthcare setting.

Chapter Four examines the philosophical and methodological positioning of the study and details the methods of data collection. The chapter begins with a critical discussion of the rationale for using a focused ethnographic approach to understanding the 'situated' experiences of ICU staff as they provide care to critically ill fat patients. The methodological approach is then presented, followed by an indepth description of the methods I used to collect and analyse my research data. I then offer a critical and reflexive discussion of how my position as a nurse researcher within my own workplace influenced the research process.

Chapter Five presents the findings of the study which conceptualises fat patients as 'misfits' in the ICU. The findings begin with a description of the ICU setting and provides an overview of the social phenomenon that existed during care situations. Following this overview each theme is presented in detail to illustrate how intensive care staff managed their private and public 'face' presentations when caring fat patients.

The discussion in Chapter Six reveals the significance of my findings in relation to the social interactions and care of 'misfits'. Throughout this chapter I provide a critical discussion of the findings in relation to current knowledge and theoretical positionings that have informed new understandings of how intensive care staff provide care to fat patients. This discussion focuses attention on how staff managed their private and professional 'face' through the use of emotional labour, behavioural regions and face-work to reduce the social awkwardness present during care.

Chapter Seven concludes this thesis where I make explicit how this research has contributed to the nursing and healthcare knowledge by providing new understandings of the specific care challenges and needs of the critically ill fat body and how staff managed their own private and professional displays of emotions when engaged in the care of socially stigmatised fat patients. An emergent dynamic- 
situational model of the insider-outsider position for nurse researchers is presented as part of the contribution to methodology. A critique of the study is provided which is followed by the implications for healthcare practice and recommendations for future research. A discussion of how the study findings contribute to the literature from the 'situated' perspectives of ICU staff in the care of fat patients concludes this chapter. 


\section{CHAPTER TWO: LITERATURE REVIEW}

\subsection{Introduction}

The care and management of extremely fat patients within healthcare is an emerging area of clinical practice and research. The unique clinical challenges that the fat body poses for the intensive care team, in conjunction with the social stigma that is attached to fatness, may have important implications for the quality of care fat patients receive during a period of critical illness. This literature review chapter has been presented as a structured narrative review where the available evidence relevant to the research topic has been synthesised to convey key messages. In this chapter I begin by providing an overview of the New Zealand intensive care context and identify the emerging research into the care and management of critically ill fat patients. Healthcare professionals' perspectives of fatness are examined and I provide a critique of the current evidence of attitudes and beliefs regarding fatness and caring for fat patients. From the perspectives of fat patients I provide a discussion of how this patient group respond to and interact with healthcare professionals when requiring healthcare provision. Theoretical perspectives that underpin stigma, prejudice and discrimination, as they apply to the healthcare setting and health literature, are examined and I draw conclusions about what is known about the influences of fat stigma on care practices.

Given the wealth and breadth of research in this field, it was important to establish the positioning of the thesis in order to determine the appropriate inclusion of current literature that would inform the research. The focus of the research was about understanding health professionals' perspectives of managing the care of fat patients and not the fat patients' perspective per se. Therefore, whilst acknowledging the very valuable and important contribution of fat studies work in regards to embodiment and gendered feminist perspectives, this body of knowledge sits firmly outside the focus of this literature review and has not been included. The clinical literature included in this review focuses only on research related to fat patients who have a BMI $\geq 40 \mathrm{~kg} / \mathrm{m}^{2}$ as the health risks, care implications, and management of this specific patient population differs to patients with BMIs $<40 \mathrm{~kg} / \mathrm{m}^{2}$. 


\subsection{Intensive care in the New Zealand context}

An intensive care unit (ICU) is defined by the College of Intensive Care Medicine of Australia and New Zealand (2011) as:

A specially staffed and equipped, separate and self-contained area of a hospital dedicated to the management of patients with life-threatening illnesses, injuries and complications, and monitoring of potentially life-threatening conditions. It provides special expertise and facilities for support of vital functions and uses the skills of medical, nursing and other personnel experienced in the management of these problems (p.1).

New Zealand intensive care units are categorised into three levels (Level I, II, III) depending on the size of the unit, the type and severity of the patient's illness, the training and expertise of staff, and support services available (College of Intensive Care Medicine of Australia and New Zealand, 2011). Level I ICU's provide immediate resuscitation and short-term cardio-respiratory support for critically ill patients and have the ability to mechanically ventilate a patient and conduct invasive cardiovascular monitoring for at least several hours. Generally patients requiring cardio-respiratory support are transferred to a level II or III unit. Predominately, level I units provide monitoring and care to 'at risk' medical and surgical patients (College of Intensive Care Medicine of Australia and New Zealand, 2011). Level II ICU's provide high standards of general intensive care for patients with complex, multisystem life support needs and have the ability to provide mechanical ventilation, renal replacement therapy and invasive cardiovascular monitoring for an unlimited period of time. Level III ICUs, are tertiary referral units for level I and II ICUs and provide comprehensive critical care services to critically ill patients by providing complex multi-system life support for an indefinite period (College of Intensive Care Medicine of Australia and New Zealand, 2011).

Currently within New Zealand there are 265 intensive beds across 29 adult ICUs and 1 paediatric ICU, which equates to approximately 6 beds per 100,0000 population (Australian and New Zealand Intensive Care Society, 2013). There are over 17,000 admissions each year to these intensive care units and of that approximately $37 \%$ are planned admissions following elective surgery with the 
remainder considered to be emergency admissions. Approximately $50 \%$ of patients will require mechanical ventilation at some point throughout their admission, which is usually between 1-3 days, and 7.7\% will die on the unit (Australian and New Zealand Intensive Care Society, 2013).

The intensive care team comprises specially trained and educated medical and nursing personnel who provide 24 hour one-to-one specialist care. Each ICU has a medical director, registered as a fellow of the College of Intensive Care Medicine, who takes overall responsibility of the unit. At all times there is at least one medical registrar exclusively rostered to the unit who has direct support from an intensivist if needed (College of Intensive Care Medicine of Australia and New Zealand, 2011). The nursing requirements of each shift is dependent on the total number of patients, and severity of patient illness. For each shift there must be a nurse-in-charge who has a post registration ICU qualification, who undertakes a supernumerary role and is responsible for the logistical management of patients, staff, service provision and resource utilisation during a shift (Morley, 2005). The staffing levels of the nursing team should be sufficiently flexible to provide 1:1 nurse-patient ratios with the ability to increase the ratio to 2:1 for very complex patients. All staff must be trained in advanced life support and at least $50 \%$ of the nursing team on any one shift should have a post registration ICU qualification (College of Intensive Care Medicine of Australia and New Zealand, 2011).

The critcal care nurse provides specialist knowledge and skills to enhance the delivery of patient-centred care within a highly technical environment (College of Intensive Care Medicine of Australia and New Zealand, 2011; Morley, 2005). The role of the intensive care nurse is to provide care to patients and their families, using specific clinical knowledge and skills that underpin the technological management of complex equipment which supports the patient's organ failure (Ä̈̈ri, Tarja, \& Helena, 2008). To do this, nurses continually monitor and assess the patient's condition, and make clinical decisions that respond to abnormal and urgent situations, in conjunction with medical and allied health staff (Ääri et al., 2008). 
The patient population in ICU is constantly changing and reflects the changing demographics of the general population. Patients cared for in the ICU are increasingly older and have more co-morbidities (Dawson, 2006). For the fat patient, typical reasons for admission to ICU include: elective or acute post-operative care, trauma ${ }^{6}$, treatment of a primary condition, complications due to co-morbid diseases and treatment of relatively minor insults to the body that lead to major health consequences (Jamadarkhana et al., 2014).

\subsubsection{Fat patients in the ICU}

Critically ill fat patients invariably have compromised dysfunction of one or more organ systems, in addition to deranged pathophysiology due to their excess fat (Bajwa, Sehgal, \& Bajwa, 2012). This fat specific pathophysiology and physical size of the patient presents many unique challenges for the entire ICU team. These challenges require many aspects of care to be modified in order to achieve good patient outcomes (Gallagher-Camden, 2006; Jamadarkhana et al., 2014; Varon \& Marik, 2001). Despite these issues that the fat body poses, clinical research addressing the care of critically ill fat patients remains scarce (Bajwa et al., 2012; Hurst, Blanco, Boyle, Douglass, \& Wikas, 2004; Varon \& Marik, 2001). Table 2 provides examples of the emerging literature regarding the care of this patient population.

Table 2: Current areas of research in the care of critically ill fat patients

\begin{tabular}{|c|c|c|}
\hline $\begin{array}{l}\text { Emerging areas of } \\
\text { knowledge }\end{array}$ & $\begin{array}{l}\text { Examples of key } \\
\text { papers }\end{array}$ & $\begin{array}{l}\text { Key messages to emerge from the } \\
\text { literature }\end{array}$ \\
\hline $\begin{array}{l}\text { Physiological changes } \\
\text { caused by fatness } \\
\text { Respiratory system } \\
\text { Altered gas exchange, lung } \\
\text { volumes, compliance and } \\
\text { resistance, oxygen } \\
\text { consumption and carbon } \\
\text { dioxide production, and } \\
\text { efficiency and work of } \\
\text { breathing. } \\
\text { Cardiovascular system } \\
\text { Altered cardiac function, } \\
\text { blood volume, and electrical } \\
\text { conduction. }\end{array}$ & $\begin{array}{l}\text { Adams and Murphy } \\
(2000) \\
\text { Bajwa et al. (2012) } \\
\text { El-Sohl (2004) } \\
\text { Davidson, Kruse, Cox, } \\
\text { and Duncan (2003) } \\
\text { Huschak, Busch, and } \\
\text { Kaisers (2013) } \\
\text { Jamadarkhana et al. } \\
\text { (2014) } \\
\text { Lewandowski and } \\
\text { Lewandowski (2011) }\end{array}$ & $\begin{array}{l}\text { The physiological changes that occur with } \\
\text { increasing fatness substantially impacts on } \\
\text { the way in which fat patients respond to } \\
\text { intensive care interventions that support } \\
\text { organ failure. } \\
\text { This is associated with increased length of } \\
\text { ICU stay, and higher rates of } \\
\text { complications, morbidity and mortality. }\end{array}$ \\
\hline
\end{tabular}

${ }^{6}$ Trauma refers to bone and soft tissue injury, or infection (Jamadarkhana, Mallick, \& Bodenham, 2014). 


\begin{tabular}{|c|c|c|}
\hline $\begin{array}{l}\text { Gatrointestinal /endocrine } \\
\text { system } \\
\text { Altered metabolism }\end{array}$ & $\begin{array}{l}\text { Varon and Marik } \\
(2001)\end{array}$ & \\
\hline $\begin{array}{l}\text { Challenges to ICU } \\
\text { Intervention } \\
\text { Respiratory system } \\
\text { Airway management } \\
\text { Mechanical ventilation (MV) }\end{array}$ & & $\begin{array}{l}\text { Anatomical changes to the airway make } \\
\text { endotracheal intubation and tracheostomy } \\
\text { placement difficult. This increases the risks } \\
\text { of accidental dislodgement and occlusion. } \\
\text { Reduced physiological reserve impairs MV } \\
\text { leading to longer MV hours. }\end{array}$ \\
\hline $\begin{array}{l}\text { Assessment, invasive access } \\
\text { and monitoring } \\
\text { Vascular access } \\
\text { Radiological imaging } \\
\text { Nutrition } \\
\text { Feeding regimes } \\
\\
\text { Drug administration } \\
\text { Pharmacokinetic drug } \\
\text { properties }\end{array}$ & $\begin{array}{l}\text { Adams and Murphy } \\
\text { (2000) } \\
\text { Bajwa et al. (2012) } \\
\text { Davidson et al. (2003) } \\
\text { El-Sohl (2004) } \\
\text { Huschak et al. (2013) } \\
\text { Jamadarkhana et al. } \\
\text { (2014) } \\
\text { Lewandowski and } \\
\text { Lewandowski (2011) } \\
\text { Varon and Marik } \\
\text { (2001) }\end{array}$ & $\begin{array}{l}\text { Fat obscures anatomical landmarks making } \\
\text { vascular access more technically } \\
\text { challenging and diagnosis from radiological } \\
\text { imaging less certain. } \\
\text { Excess fat stores and altered metabolism } \\
\text { affect the way in which enteral feeds are } \\
\text { metabolised increasing the complexity of } \\
\text { nutritional requirement calculations and } \\
\text { risk of protein malnutrition. } \\
\text { Physiological changes affect the } \\
\text { pharmacokinetic properties of drugs } \\
\text { increasing the complexity of drug dosing } \\
\text { and uncertainty of correct doses and } \\
\text { therapeutic ranges. }\end{array}$ \\
\hline $\begin{array}{l}\text { Complications of fatness } \\
\text { Airway patency } \\
\text { Tube dislodgment }\end{array}$ & $\begin{array}{l}\text { Davidson et al. (2003) } \\
\text { El-Sohl (2004) } \\
\text { El-Solh and Jaafar } \\
\text { (2007) } \\
\text { Jamadarkhana et al. } \\
\text { (2014) } \\
\text { Lewandowski and } \\
\text { Lewandowski (2011) }\end{array}$ & $\begin{array}{l}\text { Unsecured airways increase the risk of life- } \\
\text { threatening events due the difficulty of re- } \\
\text { intubation and establishing a patent airway. } \\
\text { Decreased vascularity in fat deposits, } \\
\text { increased pressure from the weight of the } \\
\text { body, and reduced mobility increases the } \\
\text { risk of skin ulceration and impaired wound } \\
\text { healing. }\end{array}$ \\
\hline $\begin{array}{l}\text { Practical implications of the } \\
\text { patient's physical size } \\
\text { Barriers to care } \\
\text { Resources } \\
\text { Resource requirement and } \\
\text { utilisation } \\
\text { Human resources } \\
\text { Specialised equipment }\end{array}$ & $\begin{array}{l}\text { Davidson et al. (2003) } \\
\text { Gallagher-Camden } \\
(2006) \\
\text { Hurst et al. (2004) } \\
\text { Jamadarkhana et al. } \\
(2014) \\
\text { Rose et al. (2008) } \\
\text { Winkelman and } \\
\text { Maloney (2005) }\end{array}$ & $\begin{array}{l}\text { Inadequate infrastructure, equipment and } \\
\text { training impact on the quality and ability to } \\
\text { provide care. } \\
\text { Increased staffing numbers, the need for } \\
\text { and use of specialised equipment to } \\
\text { conduct cares makes caring labour } \\
\text { intensive and time consuming. } \\
\text { The immobility and weight of the patient in } \\
\text { combination with the frequency of the need } \\
\text { to inspect, reposition and transfer the } \\
\text { patient increases the risk of staff injury. } \\
\text { The availability of appropriate equipment } \\
\text { further impacts on the risks to staff safety. }\end{array}$ \\
\hline
\end{tabular}




\begin{tabular}{|c|c|c|}
\hline $\begin{array}{l}\text { Improving the quality of } \\
\text { care } \\
\text { Best practice } \\
\text { Guidelines } \\
\text { Protocols } \\
\text { Manual handling }\end{array}$ & $\begin{array}{l}\text { Hurst et al. (2004) } \\
\text { McGinley and Bunke } \\
(2008)\end{array}$ & $\begin{array}{l}\text { Improved service delivery to fat patients } \\
\text { through the identification of appropriate } \\
\text { bariatric resources and equipment that } \\
\text { address both physiological and } \\
\text { psychological well-being in the intensive } \\
\text { care setting. }\end{array}$ \\
\hline
\end{tabular}

As illustrated in Table 2, much of the intensive care literature, with regards to fat patients, is medically orientated and focuses on the management of fat specific physiological changes during episodes of critical illness. There is limited but emerging evidence to support the physical and practical implications of care provision in ICU. Underpinning this emerging nursing knowledge is a considerable body of literature that addresses the care of elective bariatric surgical patients from a practical perspective (Drake, McAuliffe, \& Edge, 2012; Grindel \& Grindel, 2006; Ide, Farber, $\&$ Lautz, 2008). This literature focuses on the types of resources, training, and skills that are needed to provide safe and high quality patient care. To date, it is this nursing literature that is used to inform ICU nursing practices.

From the ICU literature it is evident that the physical size of the fat patient can complicate even the most basic intervention which can have significant consequences for both staff and patients. For nurses, lifting, turning, and repositioning totally dependent fat patients is logistically challenging, physically hard work, labour intensive, time consuming, and can predispose staff to injury (Gallagher-Camden, 2006). For patients, the difficulties encountered by staff in providing adequate patient activity and mobility can lead to additional patient care issues and complications that are exacerbated by their altered physiology (Gallagher-Camden, 2006). Despite these frequently cited concerns and consequences, the current intensive care literature provides little, if any, in-depth detail on the ways in which the size and shape of the fat body challenges everyday ICU care practice.

\subsection{Healthcare professionals' perspectives of fatness}

Attitudes and beliefs reflect our own perspectives of how we see, interpret, make sense, and respond to the world around us (Eiser, 1997). This has important implications for how healthcare professionals respond to the patients in their care, particularly those who have socially stigmatised health conditions. The following 
section explores the attitudes and beliefs of healthcare professionals in regards to fatness and how this may influence the care of fat patients.

\subsubsection{Medical professionals' attitudes and beliefs about fatness}

One of the first surveys indicating that healthcare professionals were not exempt from weight bias was undertaken by Maddox and Liederman (1969) in a study that examined the training, experience, and attitudes of physicians in the management of fat patients. The survey of 100 physicians, senior registrars and medical clerks working in a medical clinic identified that physicians exhibited negative attitudes towards fat patients. Physicians described them as unintelligent, unsuccessful, inactive, weak-willed, awkward and ugly. Physicians preferred not to treat their patients for their fatness as they did not expect the patients to comply with the management plan prescribed. Personal experience with fat patients was reported to be more influential than formal medical training on the information sourced regarding fatness and how to manage it. From these findings, Maddox and Liederman (1969) concluded that the physicians reactions to fat patients and decisions regarding management were substantially influenced by the evaluation of the fat person as morally weak and aesthetically unpleasing. They further suggested that physicianpatient interactions reflected a multifaceted combination of medical fact and sociocultural values whereby fatness, despite its medical implications was viewed “first and foremost as a social disability" (Maddox \& Liederman, 1969, p. 214).

In a later study, Klein, Najman, Kohrman, and Munro (1982) found that one third of physicians identified fatness as the fourth highest ranked condition, after drug addiction, alcoholism and mental illness, to which they responded negatively, with feelings of discomfort, reluctance and dislike. Additionally, the physicians reported an association of fatness with poor hygiene, non-compliance, dishonesty and hostility which Klein et al. (1982) suggested reflected the Protestant work ethics held by physicians of self-discipline, perserverance in times of adversity, and achievement. However, these beliefs, that fat individuals were unpleasant and dishonest, were challenged in a later study by Foster et al. (2003), where it was reported that less than $10 \%$ of physicians held these views. 
Beliefs about the causes of fatness (Foster et al., 2003), patient compliance with weight management strategies (Galuska, Will, Serdula, \& Ford, 1999; M. Hebl $\& \mathrm{Xu}, 2001$ ), and the effectiveness of weight loss programmes (Foster et al., 2003) have been identified as significantly influencing the overall attitudes and practices of doctors during consultations with fat patients. A study that examined the consultation processes of 122 primary care physicians which assessed the time spent with patients, tests and procedures ordered and general attitudes demonstrated that physicians' attitudes and treatment practices were siginficantly affected by the patient's weight (Hebl \& Xu, 2001). Physicians viewed fat patients more negatively than slim patients, felt they needed to give stricter medical advice to fatter patients, and despite requesting more medical tests that directly related to assessing co-morbid risks of fatness they reported that they would spend less time with fat patient during consultations. Physicians predicted that fatter patients would be less likely to follow their advice and therefore were a waste of their time. From their assessment of patients, physicians percieved fat patients to be unhappy and unstable and they believed that they would benefit from psychological counselling (Hebl \& Xu, 2001). These findings have been supported by other research where Foster et al. (2003) who examined 622 primary care physicians' attitudes about fatness and its treatment reported that more than $50 \%$ of physicians felt that fat patients were non-compliant with treatment. Physician's rated weight loss treatment as significantly less effective than other chronic condition therapies. However, despite this poor success rate, $54 \%$ physicians reported that they would spend more time working with patients on weight management issues if their time was appropriately reimbursed (Foster et al., 2003).

\subsubsection{Fat specialists' attitudes and beliefs about fatness}

Healthcare professionals who specialise in fat research and clinical management of fatness have demonstrated weight bias (Schwartz et al., 2003; Teachman \& Brownell, 2001). The attitudes and beliefs of 84 healthcare professionals who were current or likely prescribers of weight reduction medications attending an obesity education meeting were assessed using implicit ${ }^{7}$ and explicit ${ }^{8}$ anti-fat bias

${ }^{7}$ Implicit anti-fat bias is measured using an implicit associations test (IAT) which assesses unconscious and automatic attitudes and stereotypes primarily related to prejudice (Teachman \& Brownell, 2001). 
measures (Teachman \& Brownell, 2001). Implicit anti-fat pro-thin bias was found for both attitude and stereotype measures. From this finding, Teachman and Brownell (2001) concluded that the implicit anti-fat bias was 'strong' among healthcare professionals. However, from their study it is uncertain what was meant by the term 'strong' as clarity around this finding was not provided. For example, it was not clear if 'strong' related to the strength of the bias on the measurement scales (i.e. the more positive the calculated score the stronger the bias), or that any positive score suggested a 'strong' bias, or that 'strong' indicated that more healthcare professionals had positive scores (indicating an anti-fat pro-thin bias) than negative scores. Review of the explicit attitudes and beliefs measures indicated that there was minimum evidence to support that healthcare professionals held explicit anti-fat pro-thin bias. All the explicit scores were close to the neutral point of the measurement scale. Healthcare professionals did not endorse evaluations of fat people as being 'bad' but did support the belief that fat people were less motivated than thin people. Reasons cited for this difference in attitudes and beliefs between memory associated classification and self-reported feelings were that individuals may not be aware of their own biases, or that they wanted to appear equitable and respond in socially desirable ways (Teachman \& Brownell, 2001).

In the same study, Teachman and Brownell (2001) further compared the results of healthcare professionals to that of the general population, in this case beach goers in Connecticut, New England ${ }^{9}$, and found that bias was less in healthcare professionals. Proposed reasons for this observed difference was that healthcare professionals in the study had chosen to work with fat patients and therefore, would have more positive attitudes. Secondly, it was indicated that healthcare professionals, by the very nature of their professional values, had increased empathy towards fat patients and more comprehensive knowledge about fatness (Teachman \& Brownell, 2001).

${ }^{8}$ Explicit anti-fat bias is measured using comparison scales of feelings towards fat and thin people which assesses consciously expressed attitudes and beliefs (Teachman \& Brownell, 2001).

${ }^{9}$ The general population sample data was taken from a concurrent study being conducted by Teachman, Gapinski, and Brownell (2001) which was later published by Teachman, Gapinski, Brownell, Rawlins, and Jeyaram (2003) as part of a larger study. 
A study examining weight bias of 389 fat specialists by Schwartz et al. (2003), using the same implicit and explicit IAT instrument as the previous study, established that these health professionals exhibited weight bias consistent with findings from medical and general population studies. However, participants (31\%) who worked directly with fat patients exhibited less anti-fat bias on the implicit associations test (IAT) lazy-motivated measure than those with no direct patient interaction. Equally, participants who worked directly with fat patients in clinical practice reported significantly more positive professional and personal experiences with fat individuals, and demonstrated higher levels of understanding of the fat person's experiences of fatness than their non-clinical counterparts (Schwartz et al., 2003).

Professional and personal experiences of working with fat patients were associated with lower explicit bias but not implicit bias (Schwartz et al., 2003). These findings are important for two reasons. Firstly, positive experiences with fat patients might lower explicit attitudes or alternatively positive attitudes might lead to more positive experiences (Schwartz et al., 2003). Secondly, positive experiences appeared not to influence implicit attitudes suggesting that these implicit associations are not linked as closely to how individuals actually interact with fat people (Schwartz et al., 2003). Furthermore, implicit attitude tests may overestimate anti-fat bias (Fabricatore, Wadden, \& Foster, 2005). This work suggests that the implicit bias amongst health professionals should not be used as a primary indicator to suggest poorer treatment of fat patients (Schwartz et al., 2003).

\subsubsection{Nurses' attitudes and beliefs about fatness}

Nurses caring for fat patients have exhibited similar weight bias to that of the medical profession (Bagley, Conklin, Isherwood, Pechiulis, \& Watson, 1989; Brown, 2006; Culbertson \& Smolen, 1999; Hoppe \& Ogden, 1997; Maroney \& Golub, 1992; Mercer \& Tessier, 2001, Petrich, 2000). Nurses evaluated fat patients more negatively than normal weight patients and frequently assigned a range of negative stereotypical attributes similar to their medical colleagues such as unmotivated (Hoppe \& Ogden, 1997; Mercer \& Tessier, 2001), non-compliant (Hoppe \& Ogden, 1997), lazy (Culbertson \& Smolen, 1999; Maroney \& Golub, 1992), lacking self-control 
(Maroney \& Golub, 1992; Petrich, 2000) and being unattractive (Maroney \& Golub, 1992; Peternelj-Taylor, 1989).

Attitudes of nurses towards engaging in the care of fat patients have changed over the last 25 years. In the late 1980s Bagley et al. (1989) developed an attitudes towards obesity and obese patients (ATOOP) scale which has subsequently been used in many other studies to assess nurses' attitudes regarding fat patients (Culbertson \& Smolen, 1999; Garner \& Nicol, 1998; Maroney \& Golub, 1992). In Bagley et al.'s (1989) original study, which assessed 107 female nurses' attitudes towards fat patients, they reported that one in eight $(12.3 \%)$ nurses indicated that they would avoid touching fat patients and one in four $(24.3 \%)$ nurses were repulsed when caring for those individuals. Subsequent studies, using either the ATOOP scale or similar attitudinal survey tools, have reported less negative findings. In a study by Culbertson and Smolen (1999) which examined the attitudes of 73 nurses towards fat patients, using the ATOOP scale, they reported that only $5 \%$ of nurses indicated that they 'would rather not touch an obese adult'. Similarly, Brown, Stride, Psarou, Brewins, and Thompson (2007) examined the practices, beliefs and attitudes of 564 primary care nurses in respect to weight management and found that only $4.3 \%$ of nurses felt repulsed by fat patients. Likewise, there has been a consistent and substantial reduction over the previous 20 years in the percentage of nurses who would prefer not to care for fat patients if they had a choice, from $31.3 \%$ (Maroney \& Golub, 1992) to $17.7 \%$ (Poon \& Tarrant, 2009). Both these studies used or adapted the ATOOP scale in their survey of nurses' attitudes. An often cited reason for nurses being unwilling to care for or avoiding fat patients is that they are physically demanding and exhausting (Culbertson \& Smolen, 1999; Maroney \& Golub, 1992; Poon \& Tarrant, 2009).

In addition to the reduction in the negative attitudes towards caring for fat patients, studies have indicated that nurses' attitudes were not always purely negative but mixed and ambivalent (Mercier \& Tessier, 2001; Petrich, 2000; Wright, 1998; Zuzelo \& Seminara, 2006). More positive attitudes cited were: concern for and empathy with fat patients (Petrich, 2000; Wright, 1998; Zuzelo \& Seminara, 2006), worry about their victimisation (Petrich, 2000; Zuzelo \& Seminara, 2006), and being willing to work with fat patients (Mercer \& Tessier, 2001). These studies all adopted a 
qualitative or mixed methods approach such as semi-structured interviews or surveys and questionnaires that included open ended questions to explore perceptions. Both these approaches allowed participants to articulate in their own words their own personal perspectives on fatness and caring for this patient population.

Positive attitudes towards caring for fat patients was reported in a study by Zuzelo and Seminara (2006) of 119 registered nurses' attitudes across different healthcare settings. The study examined the attitudes of nurses using the ATOOP assessment tool and open ended questions that explored care interactions. There were only six items on the 28-item ATOOP scale where the mean score produced a negative response. These were related to the challenging physical care demands and perceived beliefs about lifestyles choices of fat adults, which were consistent with previous studies (Culbertson \& Smolen, 1999; Maroney \& Golub, 1992). Equally, there were four items on the scale where the mean score identified that nurses were not repulsed or disgusted when caring for fat patients, and did not mind touching them, suggesting that those nurses who had strong reactions to the fat body were in the minority. Again these findings are consistent with the positive shift in attitudes in regards to engaging in physical care activities identified in the studies by Brown and Thompson (2007), Culbertson and Smolen (1999), and Poon and Tarrant (2009). One possible explanation for this positive trend is that caring for fat patients is now a normal part of everyday care practices where through familiarity with fat bodies the perceived associated repulsion has diminished.

In addition to the ATOOP assessment of attitudes, participants were asked to comment on their initial thoughts and feelings when they entered the room of a fat patient for the first time (Zuzelo \& Seminara, 2006). Thematic analysis of the data identified eight themes related primarily to personal and patient safety, recognising unique and complex care needs, and ensuring care was delivered respectfully and without prejudice (Zuzelo \& Seminara, 2006). Only one theme, 'feeling astounded', indicated that staff had feelings of blame towards the fat person, with one respondent commenting: "I think, how did this person allow him/herself to get this heavy?" (Zuzelo \& Seminara, 2006, p. 71). Three of the themes specifically focused on how nurses attempted to ensure that a positive interaction or connection with the patient 
occurred on first encounters. Nurses emphasised the importance of treating fat patients no differently to other patients, being diligent about monitoring ones own facial expressions and body language so not to unintentionally insult or offend, and feeling empathetic towards the difficult situation the fat patient may face during their hospital stay. This study provides some brief insight, albeit self-reported, into how nurses managed their feelings regarding fatness and how they behaved when engaging in patient care.

Belief about the causes of fatness, consequences on health and its prevention were found to significantly inform the overall attitudes of nurses caring for fat patients. The most dominant beliefs held across studies were that fatness was a result of poor lifestyle choices (Culbertson \& Smolen, 1999; Hoppe \& Ogden, 1997; Poon \& Tarrant, 2009), was preventable and treatable (Culbertson \& Smolen, 1999; Hoppe \& Ogden, 1997). Culbertson and Smolen (1999) found that the majority of registered nurses felt that fat patients had poor food selections and could lose weight if they changed their dietary habits, and that being fat could be prevented by individuals exercising self-control over their eating. Underpinning these beliefs is the idea that fatness is largely perceived to be an imbalance of food intake and energy expediture caused by overeating and being lazy and inactive (Poon \& Tarrant, 2009).

Lifestyle factors have been deemed more important than biological factors in the reasons why people are fat (Hoppe \& Ogden, 1997; Poon \& Tarrant, 2009). Similarly, the failure of patients to lose weight has been attributed to non-compliance with management plans and lack of motivation (Hoppe \& Ogden, 1997; Mercer \& Tessier, 2001). When these attributes are assigned to fat patients, Mercer and Tessier (2001) reported that it led primary care nurses to feel ambivalent and apathetic when working with them to lose weight. This is consistent with previous medical findings reported by Maddox and Liederman (1969) in their study of primary care physicians.

A more expansive understanding of causative factors of fatness, than those cited above, was identified in a study of primary care nurses (Brown \& Thompson, 2007). Nurses considered fatness to be a multifaceted phenomenon where a complex range of wider factors were responsible for people becoming fat. Nurses demonstrated 
a greater awareness of the broader environmental, cultural and economic factors that impacted on eating and physical activity behaviours. For example, how family and personal circumstances could affect lifestyle choices that led to individual's becoming fat. In concurrence with previous studies (Culbertson \& Maloney, 1999; Hoppe \& Odgen, 1997; Poon \& Tarrant, 2009), genetical and medical conditions were not considered major causative factors of fatness.

When nurses discussed their beliefs with other people, Brown and Thompson (2007) noted that the 15 nurses interviewed in their study placed particular emphasis on conveying empathy and being non-judgemental and were cautious in assigning stereotypes and giving overly simplistic explanations. For example, in previous studies, fat patients were often identified as 'unmotivated' on various belief scales (Hoppe \& Ogden, 1997; Mercer \& Tessier, 2001), however, participants in the Brown and Thompson (2007) study acknowledged that motivation changed over time and therefore could not be used as a generalised statement. Despite this more empathetic perspective on the impact that external factors and pressures have on becoming fat, there was still the belief that patients had some degree of personal responsibility for being fat. Although where that balance lay varied between participant responses (Brown \& Thompson, 2007). This more expansive understanding of beliefs regarding fatness may reflect a change in general understandings of the causative factors of fatness in contemporary society or simply that the qualitative approach of this study allowed for nurses to be more expressive and accurate in articulating their beliefs.

Beliefs about the physical health consequences of being fat underpinned many attitudes held by nurses (Brown et al., 2007; Petrich, 2000; Wright, 1998). Ninety percent of nurses in a small qualitative study of 10 registered nurses that investigated acceptable body sizes of women felt that being overweight posed serious health problems, primarily coronary heart disease, breathing difficulties and diabetes (Wright, 1998). Psychological, emotional and social consequences of being fat was not cited as a health consequence by any of the participants. Only one nurse stated that being overweight did not relate to ill-health (Wright, 1998). This strong belief in the consequences that fatness is unhealthy has been supported in later studies (Brown 
et al., 2007; Petrich, 2000), where $58.5 \%$ of nurses believed that most health problems of a fat patient were a result of being fat (Brown et al., 2007).

\subsubsection{Demographic variables of nurses}

Gender, age, BMI, and professional experience of nurses have been shown to have some affect on the nurse's overall attitudes towards fat patients (Bagley et al.,1989; Brown \& Thompson, 2007; Culbertson \& Smolen, 1999; Garner \& Nicol, 1998; Hoppe \& Ogden, 1997; Nordholm, 1980; Peternelj-Taylor, 1989; Westbrook \& Mitchell, 1979; Wright, 1998; Young \& Powell, 1985). However, the extent to which these variables can predict weight bias is a contentious point due to the inconsistent findings throughout the nursing studies (Brown, 2006).

\section{Gender}

The gender of the nurse appeared to have some influence on the attitudes towards fat patients. In a study by Young and Powell (1985) which explored how fatness influenced clinical judgements of 120 mental health workers, male participants were found to be much less severe in their assessment of fat patients than their female counterparts. Likewise, when Garner and Nicol (1998) specifically compared 23 male and 45 female nurses' attitudes towards fat patients they identified that different aspects of care evoked different attitudes depending on the nurses' gender. For example, only female nurses (18\%) reported repulsion in bathing fat patients, whereas male nurses reported that this care activity did not evoke feelings of disgust. Despite this, the study found that there was no significant difference in the overall attitudes of male and female nurses. This finding was reflected in the perceptions of fat patients who did not perceive there to be any difference in the attitudes of male and female nurses towards their care (Garner \& Nicol, 1998).

Not only did the gender of the nurse influence attitudes so too did the gender of the fat patient. Studies in the 1970s and 1980s which examined the effects of patient weight and gender on nursing perceptions of their patients demonstrated that female healthcare professionals evaluated fat males more negatively than fat female patients (Nordholm, 1980; Peternelj-Taylor, 1989; Westbrook \& Mitchel, 1979). A 
reason offered for this finding was that female health professionals held an allegiance to other females (Peternelj-Taylor, 1989).

In contrast, a later study conducted by Wright (1998) provided considerable evidence to suggest that female patients were percieved more negatively and treated in more discriminatory ways than male patients by healthcare professionals. Nine out of the ten nurses in the study had observed instances of unfair and unkind treatment of female patients by male doctors, such as denying treatment or joking about their body size. This joking did not occur with male patients, who one nurse reported was because 'it seems to be more acceptable for a man to be big...//...there doesn't seem to be as much comment about male patients'(Wright, 1998, p. 312). From these studies, it appears that the gender of the nurse and the patient does have some influence on attitudes and beliefs about caring for fat patients. However, the varied focus of this research and methodologies used limits the conclusions that can be drawn to the specific context of each study.

\section{Age}

Although, the age of the nurse has been frequently reported as being influential on the attitudes about fatness and fat patients (Bagley et al., 1989; Culbertson \& Smolen, 1999; Young \& Powell, 1985), the evidence as to how and why this is so remains unclear (Brown, 2006). Young and Powell (1985) found that younger mental health clinicians were more severe in their judgements of fat patients than older clinicians. Similarly, Culbertson and Smolen (1999) identified that as the age of the nurse increased they tended to have less negative attitudes or stigma towards managing the care of fat patients. However, Bagley et al. (1989) reported that older nurses held less favourable attitudes than their younger counterparts. In all of the studies, the authors did not provide any explanations to support these findings.

\section{Body mass index}

Understanding the influence of nurses' body size on attitudes towards fat patients has been examined in numerous studies (Brown \& Thompson, 2007; Culbertson \& Smolen, 1999; Hoppe \& Ogden, 1997; Young \& Powell, 1985). One of the first studies to investigate this relationship reported that those with higher BMI's 
were more severe than normal weight staff in their clinical judgements of fat patients (Young \& Powell, 1985). Similarly, Brown and Thompson (2007) found that although nurses with higher BMIs reported they could empathise more strongly with fat patients and draw on their own personal experiences during weight loss discussions, there was a propensity to be more critical and judgemental of fat people (Brown \& Thompson, 2007). Likewise, both Hoppe and Ogden (1997) and Culbertson and Smolen (1999) reported that nurses with higher BMIs held similar overall attitudes towards fat patients as their non-fat colleagues. These findings, that increasing BMI did not produce more positive attitudes towards fat people, were consistent with other studies conducted within the general population which demonstrated that fat people were prejudiced against their own group (Crandall, 1994; Crandall \& Biernat, 1990; Crocker \& Major, 1989; Friedman et al., 2005). This lack of positive in-group bias ${ }^{10}$ infers that fat people do not share a positive and collective identity of themselves (Crandall, 1994; Friedman et al., 2005).

\section{Professional experience and education}

The evidence of the impact of years of nursing experience and professional education of the nurse on attitudes and beliefs regarding fatness and caring for fat patients is inconsistent in the literature (Brown, 2006). Two studies, both of North American nurses, used the same ATOOP scale to assess attitudes produced conflicting findings. The first study by Bagley et al. (1989) reported that nurses with more years of professional education showed more favourable attitudes towards fat patients. However, the specifics of these attitudes and details clarifying what is meant by 'more years of professional education' were not elaborated on. In contrast, Culbertson and Smolen (1999), who specifically focused their research on assessing if educational preparation and nursing experience affected attitudes, found that there was no significant difference between Bachelor of Nursing students and Master of Nursing students in terms of overall attitudes towards fat patients. The nursing educational level of the nurse did appear to affect the beliefs about fat patient's personalities and lifestyles (Culbertson \& Smolen, 1999). One limitation to their study was the narrow range of years of experience working as a registered nurse of 6-15 years which may in

\footnotetext{
${ }^{10}$ In-group bias refers to the tendency to favour one's own group more positively than other groups (Aberson, Healy, \& Romero, 2000).
} 
part account for no differences found between the two groups. When comparing the attitudes and beliefs of 352 undergraduate student nurses with 198 registered nurses in a study by Poon and Tarrant (2009), they found that student nurses demonstrated more positive attitudes towards fat patients, however, both groups were unlikely to attribute positive characteristics to fat people. These findings suggest that the amount of education a nurse receives and the years of nursing experience may not be the most influential factor in determining attitudes and beliefs related to fatness.

\subsection{Fat patients' perspectives of healthcare}

The effects of stigma on an individual can have profound implications for how that person interacts with others in social situations and participates in daily societal activities (see Section 2.5). This has important implications for how fat patients respond to and interact with healthcare professionals when requiring healthcare provision. A review of the literature addressing fat patients' experiences and perspectives of their healthcare has identified four main themes across multiple studies. These are patient ambivalence (Brown, et al., 2006; Thomas et al., 2008), the experience of stigma (Creel \& Tillman, 2011; Merrill \& Grassley, 2008; Puhl, MossRacusin, Schwartz, Brownell, 2008; Russel \& Carryer, 2013; Rogge, Greenwald, \& Golden, 2004), care and treatment avoidance (Adams, Smith, Wilbur, \& Grady, 1993; Amy, Aalborg, Lyons, \& Keranen, 2006; Drury \& Louis, 2002; Fontaine, Faith, Allison, \& Cheskin, 1998; Olson, Schumaker, \& Yawn, 1994; Russell \& Carryer, 2013), and patient satisfaction (Buxton \& Snethen, 2013; Fong, Bertakis, \& Franks, 2006; Hebl, Xu, \& Mason, 2003).

\subsubsection{Ambivalence}

A study that explored 28 fat patients' experiences and perceptions of support in primary care indicated that patients were generally ambivalent about their healthcare provision (Brown, Thompson, Tod, \& Jones, 2006). This sense of personal ambivalence was in response to the general ambivalence shown towards them by healthcare professionals when accessing and using primary care services. Patients felt that healthcare professionals were particularly ambivalent in addressing their weight issues, which led them to be reluctant to raise concerns when presenting with a current weight issue. The ambivalent nature of healthcare professionals' interactions 
with fat patients with regards to discussions about weight management has been identified in other studies that focused specifically on professional attitudes and care practices (Mercer \& Tessier, 2001; Wright, 1998). Healthcare professionals felt ambivalent and uneasy about assessing weight, initiating weight loss conversation (Wright, 1998), and working with fat patients (Mercer \& Tessier, 2001). Healthcare professionals' uncertainty in how patients will respond to discussions about their weight, combined with patient perceptions that staff were uninterested in supporting weight loss and discussing specific weight concerns, appears to have led to and perpetuated the apathy and ambivalence of both service providers and users of healthcare.

In contrast, more recent research exploring the lived experiences of 76 Australian people with fatness reported less obvious feelings of ambivalence towards them (Thomas et al., 2008). A third of patients recounted very positive experiences with healthcare professionals who had been strong advocates for their treatment and care. Equally, they considered their General Practitioners as their sole source of support and were appreciative of their time spent discussing overall health and wellbeing. Despite this, one half of participants described an experience of weightstigma by healthcare professionals (Thomas et al., 2008).

\subsubsection{The experience of stigma}

Patients feeling stigmatised by healthcare providers was frequently reported in studies that focused on fat patient perspectives of care (Brown et al., 2006; Creel \& Tillman, 2011; Merrill \& Grassley, 2008; Puhl et al., 2008; Russell \& Carryer, 2013; Thomas et al., 2008). A New Zealand study that explored the experiences of eight fat women when accessing primary health care services identified that the effects of social stigmatisation influenced how they engaged with and positioned themselves as healthcare consumers (Russell \& Carryer, 2013). Women reported a "heightened sensitivity to stigmatisation" (Russell \& Carryer, 2013, p.204) on entering the general practice setting because they were socially stereotyped as being lazy, gluttonous, ugly and a problem that needed fixing. This was evident during the consultation when general practitioners dimissed or belittled their presenting problem in favour of fore fronting their weight and body size as the diagnostic reason for their health concern. 
Furthermore, women reported being verbally abused, subject to inappropriate joking about their size, negative body language, and being treated in undignified ways by health care providers (Russell \& Carryer, 2013).

A study that explored the phenomenon of the stigmatisation of eight fat people with chronic illness indicated that the perceived actions of the nurse reinforced stigma responses of shame, marginalisation and anxiety in seeking healthcare by patients (Creel \& Tillman, 2011). Participants described the unintentional spiritual, emotional and psychological harm experienced when nurses spoke or gave non-verbal indications of their negative response to them being fat. Similarly, participants reported that nurses made assumptions about them based on their weight, such as assuming they had diabetes, didn't exercise and their illnesses were weight related. These assumptions were reinforced by how participants interpreted the way nurses' interacted with them. Furthermore, participants felt stigmatised when they perceived the care they were receiving was given reluctantly and that their illness was an inconvenience to the nurse. These perceptions of their care reinforced participants' stigma responses of shame, marginalisation and anxiety when requiring healthcare (Creel \& Tillman, 2011).

Shame was often experienced by patients when being cared for by healthcare professionals (Creel \& Tillman, 2011; Merrill \& Grassley, 2008; Thomas et al., 2008). Feelings of shame were expressed through the language the participants used to describe their interactions with nurses, in particular, feeling ashamed, embarrassed and humiliated. Shame and embarrassment were generally used to describe situations when the fat body was moved and exposed (Creel \& Tillman, 2011), whereas humiliation related to situations where nurses had spoken in derogative ways (Creel \& Tillman, 2011; Thomas et al., 2008).

Marginalisation, as a result of the stigma experienced, was manifested through perceptions of being seen as different (Creel \& Tillman, 2011; Merrill \& Grassley, 2008). Being different was reinforced by the constant reminder by nurses that fat patients needed 'special' equipment or gowns, or by the behaviour and attitudes expressed during care. One study of women's experiences of being fat, reported that 
being different because of body size led to 'feeling not quite human' particularly when patients' bodies were described and discussed in non-human terms (Merrill \& Grassley, 2008).

These types of stigma experiences have been associated with negative psychobehavioural responses such as maladaptive coping, low self-esteem (Friedman et al., 2005; Myers \& Rosen, 1999; Wadden et al., 2006), body-image distress (Friedman et al. 2005; Myers \& Rosen, 1999), depression (Friedman et al., 2005; Wadden et al., 2006), and general psychiatric symptoms (Friedman et al., 2005) such as anxiety (Friedman et al., 2005; Wadden et al., 2006). Psycho-behavioural responses in conjunction with past experiences of stigma within the healthcare setting have been identified as creating an environment of anxiety for patients (Creel \& Tillman, 2011).

\subsubsection{Healthcare avoidance}

An increase in BMI has been associated with increasing delays or avoidance of healthcare (Drury \& Louis, 2002; Fontaine et al., 1998; Olson et al., 1994; Russell \& Carryer, 2013). Avoidance or delays in accessing healthcare by fat patients were related to perceived provider attitudes (Adams et al., 1993; Amy et al., 2006; Russell $\&$ Carryer, 2013), weight focused care practices (Amy et al., 2006; Drury \& Louis, 2002), and perceptions of their own bodies (Olson et al., 1994; Russell \& Carryer, 2013). Over half of the fat women in a study by Olson et al. (1994) reported delaying or cancelling appointments with physicians because they knew they would be weighed. Only a small percentage of women $(2.6 \%)$ did not cancel their appointments but instead refused to be weighed during their consultations. Women of greater BMI were more likely to state that they delayed medical care because of embarrassment about their weight or because they did not want a lecture regarding their fatness. Similar reasons for delaying healthcare was identified by Drury and Louis (2002) who examined this relationship using the satisfaction with medical care scale (Packer, 1990). The most frequently cited reasons for weight-related delays in seeking healthcare were weight gain since the last visit (34.2\%), not wanting to be weighed (26.0\%), undressing for the consultation (13.7\%), and being told to lose weight (30.1\%) (Drury \& Louis, 2002). 
Preventative healthcare screening has been identified as a major area of healthcare avoidance by fat patients (Adams et., 1993; Amy et al., 2006; Fontaine et al., 1998; Russell \& Carryer, 2013). An increase in BMI has been positively correlated to increases in length of delay between screening visits (Amy et al., 2006). Women were less likely to seek cancer screening for procedures that required them to undress and have their bodies manually manipulated during the examination (Fontaine et al., 1998). For these reasons cancer screening, such as clinical breast examinations, gynaecological examinations and papanicolaou smears were the most frequently delayed procedures. Mammography screening was not associated with delayed screening and thought to be because it was less personally invasive and involves less direct contact with the healthcare professional conducting the study (Fontaine et al., 1998). In addition to anxieties about revealing their bodies, fat patients further delayed screening due to perceived attitudes of providers (Amy et al., 2006; Russell \& Carryer, 2013), and concerns that the medical equipment was too small to be functional (Amy et al., 2006).

The establishment of trusting and respectful relationships with primary care providers has been identified as being important to patients in accessing timely healthcare (Buxton \& Snethen, 2013; Merrill \& Grassley, 2008; Russel \& Carryer, 2013). In a study, by Buxton and Snethen (2013) of women's experiences of healthcare, participants reported that when they had a connection with their primary care physician they felt more comfortable, and less anxious and fearful of the encounter. In constrast, when participants did not have an established connection they reported disrespectful behaviour. Situations in which disrespectfulness occurred was when women required care from the Emergency Department staff and by primary care providers where there was no pre-existing relationship. In this study participants reported that they did not avoid healthcare but if they felt they were not receiving high quality care they sought another provider (Buxton \& Snethen, 2013). Similarly, women in the studies by Merrill and Grassley (2008) and Russell and Carryer (2013) identified that in situations where women trusted and spoke highly of their relationship with general practitioners and nurses, they felt encouraged to seek support with weight issues. 


\subsubsection{Patient satisfaction}

There is emerging evidence to suggest that not all encounters with healthcare professionals were negative and in fact patients reported positive experiences and were satisfied with their care provision (Buxton \& Snethen, 2013, Fong et al., 2006, Hebl et al., 2003). The examination of the relationship between BMI and patient satisfaction with healthcare providers was conducted by Fong et al. (2006) who reported that in the 12 months prior to the study fat patients were more satisfied with their healthcare provider than their normal weight counterparts. The patient satisfaction scale used in the study focused attention on the quality of provider interactions. The study identified that patient satisfaction within this fat patient population subgroup was higher with advancing age, where it was suggested that older participants may be more accepting of their weight (Fong et al, 2006). Another reason cited for this finding was that fat individuals, due to the societal stigma that they encounter, had low expectations and were pleasantly surprised by the support offered to them by the physician. Similarly, Buxton and Snethen (2013) found that patients reported positive experiences during care in situations where the primary care provider took the time to listen and get to know the patient, believe what they said, and focused on the concerns of the person.

From the healthcare literature presented it is evident that the perspectives of healthcare professionals and fat patients regarding fatness and patient care is entrenched in a social language of stigma, prejudice, and discrimination. This language was used in the descriptions of attitudes, beliefs and intended behaviours of healthcare professionals, and during descriptions by fat patients of their encounters with healthcare professionals. To make sense of these experiences requires an examination of the theoretical underpinnings of these concepts as they apply to the healthcare setting and health literature.

\subsection{Theoretical perspectives of stigma, prejudice and discrimination}

The concepts of stigma, prejudice, and discrimination has been used widely within healthcare as a way of describing how some patients, including those who are fat, are viewed less favourably than others and treated differently. These concepts are often used interchangeably within sociological and nursing literature, which has led to 
misunderstandings (Sayce, 1998), post hoc inferences (Carr \& Friedman, 2005), and hindered research and interventions to reduce stigma and discrimination (Mental Health Commission, 1997). If this is so, it has the potential to perpetuate the publicity regarding the discrimination of fat patients in healthcare, which may intensify the 'perceived" ${ }^{11}$ and 'internalised" ${ }^{12}$ stigma of the person thus, further limiting their participation in society.

There are fundamental differences in how stigma and prejudice have been theorised and researched which has led to largely separate literatures on the two concepts (Phelan, Link, \& Dovidio, 2008). Stigma research stems from the seminal work of sociologist Erving Goffman, who originally defined stigma as "a deeply discrediting attribute that links a person to an undesirable stereotype, leading other people to reduce the bearer from a whole and usual person to a tainted, discounted one" (Goffman, 1963b, p. 11). His work primarily focused on the target or person with the discrediting attribute. Subsequent research on stigma has generally focused on social processes derived from norm enforcement and disease avoidance, where individuals were considered diseased, had disabilities, or had deviant behaviours and identities (Stuber \& Meyer, 2008). Research in this field has mainly described the adverse effects of stigma on the stigmatised person and examined the cognitive elements of the stigmatiser, who endorsed the negative stereotype and behaved in a discriminating manner. This has resulted in many studies focusing on negative stereotypes and on public opinion surveys of those who are believed to stigmatise others (Stuber \& Meyer, 2008). This focus has led to interventions that are aimed at increasing empathy and altruism or enhancing the coping mechanisms of the stigmatised person (Parker \& Aggleton, 2003). Stigma models, therefore, have placed greater emphasis on the stigmatised person as the target, such as the stereotyping, identity and emotions involved (Phelan et al., 2008).

\footnotetext{
11 'Percieved' or 'felt' stigma refers to the fear of being discriminated against (Siyam'kela, 2003).

12 'Internalised' or 'self' stigma refers to the internalised behaviours of the stigmatised individual such as withdrawal or victim behaviour in response to percieved or enacted stigma (Van Brakel, 2006).
} 
In contrast, prejudice research originates from the seminal work of Gordon Allport who defined prejudice as "an aversive or hostile attitude towards a person who belongs to a group, simply because he belongs to that group, and is therefore presumed to have objectionable qualities ascribed to that group" (Allport, 1954, p. 7). His work primarily focused on the perpetrator of the hostile attitudes. Subsequent prejudice research has largely focused on social processes derived from exploitation and domination such as racism, sexism, and classism (Nelson, 2009). Research on prejudice has tended to examine the stress induced by discrimination during the interpersonal interactions between marginalised and non-marginalised groups (Stuber \& Meyer, 2008). This has resulted in many studies focusing on describing the psychological well-being of the marginalised person as well as examining both the implicit and explicit forms of bias held by the perpetrator (Stuber \& Meyer, 2008). Hence, prejudice models have tended to be centred on the social processes of the perpetrators as well as the discriminatory behaviours that occurred outside of the social interaction (Phelan et al., 2008).

Despite these differences, there are many similarities in the experiences of stigma and prejudice and include the exposure to negative attitudes, experiences of interpersonal and structural discrimination or unfair treatment, and hostility perpetrated against those that belong to the disadvantaged social group (Stuber \& Meyer, 2008). For this reason, there has been growing support to amalgamate stigma and prejudice research methodologies to enhance existing models that combine and conceptualise stigma and prejudice as "psychosocial stress in the lives of marginalised groups" (Stuber \& Meyer, 2008, p. 352). Importantly, this amalgamation still acknowledges the differences of stigma and prejudice methodologies and what they can offer to address social problems (Mental Health Commission, 1997; Phelan et al., 2008). Thus, the terms we use and the methodological approach taken, have the potential to lead to different understandings of where and with who the problem lies, and consequently how those problems are addressed in practice (Mental Health Commission, 1997; Sayce, 1998). Therefore, it is suggested that the term stigma should be used when referring to the broader processes that surround the social interactions of marginalised and non-marginalised groups, whereas, prejudice should be used to refer to attitudinal components of this process (Dovidio, Major, \& Crocker, 
2000; Phelan et al., 2008). Similarly, the term 'discrimination', which is embedded into stigma and prejudice conceptualisations, should be used to refer to how the actions of others have negatively impacted on the recipients of these behaviours (Mental Health Commission, 1997). This suggested usage of the terms stigma, prejudice, and discrimination have been adopted in this thesis as a way of making sense of the literature presented on fatness.

\subsubsection{Fat Stigma}

It is frequently reported that people who are fat are stigmatised in society (Brownell \& Puhl, 2003; Brownell, Puhl, Schwartz, \& Rudd, 2005; MacLean et al., 2009; Puhl \& Heuer, 2010). With new and emerging understanding and conceptualisation of stigma it is less clear if, how, and to what extent others stigmatise fat people, and the impact of that stigma on the lives of fat individuals (Link \& Phelan, 2001). Since Goffman's (1963b) work, research on stigma has been prolific, leading to new conceptualisations (Link \& Phelan, 2001), and accounts of its negative impact on those who are stigmatised (Creel \& Tillman, 2011; Friedman et al., 2005; Merrill \& Grassley, 2008). Stigma has been applied to many different illnesses and circumstances such, as HIV/AID sufferers (Froman \& Owen, 2001; Siyam'kela, 2003; UNAIDS, 2000), leprosy (Cross, 2006; Heijnders \& Van Der Meij, 2006; Parker \& Aggleton, 2003), mental health patients (Abbey et al., 2011; Link, Castille, \& Stuber, 2008; Link, Yang, Phelan, \& Collins, 2004), gay families (Goldberg \& Smith, 2011; Robitaille \& Saint-Jacques, 2009), welfare recipients (Stuber \& Schlesinger, 2006), smokers (Stuber, Galea, \& Link, 2008), and fat people (Brownell \& Puhl, 2003, Brownell et al., 2005; Puhl \& Heuer, 2009, 2010; Stoneman, 2012).

The concept of stigma has been found to be ill-defined by researchers, defined in different ways by different researchers, and its application to the field of study is significantly varied (Link \& Phelan, 2001). This lack of consensus, and varied applications using different theoretical orientations, has led to multiple meanings within different disciplines and society itself making stigma research and its understanding of fat stigma within health challenging. A new and widely accepted conceptualisation of stigma has been developed which incorporates the major themes and interpretations currently used and applied in stigma research (Link \& Phelan, 
2001, 2006). It is suggested that stigma exists when labelling, stereotyping, separation, discrimination causing status loss, occur within a social, economic and political power structure. The importance of power in this conceptualisation is that it allows for "the identification of differentness, the construction of stereotypes, the separation of labelled persons into distinct categories, and the full execution of disapproval, rejection, exclusion, and discrimination" (Link \& Phelan, 2001, p. 367). This added component of discrimination and power has been used in research on HIV/AIDS stigma (Parker \& Aggleton, 2003) and mental health stigma (Abbey et al., 2011).

\subsubsection{Theories of fat stigma}

Attribution theory is the primary theoretical model used to explain fat stigma (Puhl, Heuer, \& Brownell, 2010). Attribution theory suggests that individuals attempt to understand other people's behaviour by searching for information that will provide a reasonable explanation or cause (Puhl \& Brownell, 2003). When encountering a stigmatised condition, such as fatness, individuals attempt to search for its cause and form reactions to that person, using causal information. The idea that people get what they deserve in life is central to attributional explanations of fat stigma (Crandall, 1994). The perceptions of controllability and personal responsibility form the basis for which blame is attributed to fat people (Puhl et al., 2010). The social ideologies and moral stances of individualism, Protestant work ethic, and 'just world bias' are the most commonly cited explanations for the blame attributed to fat stigma.

Individualism has been the most theoretically developed and empirically sound basis for rationalising why fat is particularly stigmatised in western societies (Crandall \& Biernat, 1990; Crandall, 1994; Crandall \& Martinez, 1996). Individualism emphasises the notion that people are responsible for the situations in which they live and therefore get what they deserve according to their individual efforts. The common perception that weight gain and loss is under the control of the individual is an important concept in understanding fat stigma. In this context, it is believed that the individual is personally responsible for their weight and therefore fatness is associated with negative personal traits of laziness, lack of self-discipline and passivity (Puhl \& Brownell, 2001). 
Both the Protestant work ethic and 'just world bias' resemble and share similar moral positionings as individualism, which all focus on personal efforts (Puhl \& Brownell, 2003). The Protestant work ethic represents beliefs that hard work, determination and perserverence leads to achievements in life. Therefore, a high value is placed on self control and blaming victims for not succeeding (Crandall, 1994). Similarly, the 'just world bias' depicts the beliefs that a person's actions always bring morally fair and fitting consequences for that person, therefore personal efforts are rewarded (Dion \& Dion, 1987; Lerner, 1980). This belief is associated with positive bias towards successful individuals and blame for those who do not succeed (Lerner, 1980). Individuals who hold this belief have a propensity to associate positive qualities with physical attractiveness, whereby they have increased respect for physically attractive people and decreased liking for others (Dion \& Dion, 1987).

\subsubsection{Measuring stigma}

Measuring stigma has been identified as both a difficult (Lewis, Cash, Jacobi, \& Bubb-Lewis, 1997) and contentious issue, particularly in sociology, due to the varied perspectives held on the notion of 'measurement' (Scambler, 2009). However, Link et al. (2004) have argued that when considering health-related stigma (in their case mental illness) there is a need to accurately observe and measure it in order to advance scientific understandings. The purpose of stigma assessment is to develop a greater understanding of the determinants and dynamics of stigma, be able to measure the extent and severity of that stigma on a specific group, and be able to monitor change over time (Van Brakel, 2006). Within health, the impact of policies, initiatives and funding into stigma reduction strategies and impact on the well-being of the stigmatised person can be evaluated through the use of robust measurements tools. The sound measurement of anti-fat attitudes is paramount to better understanding fat prejudice and discrimination in society (Lewis et al., 1997).

Five categories have been identified for assessing health-related stigma which address different components of the stigma process (Van Brackel, 2006). These are: 
attitudes ${ }^{13}$ towards the affected person; 'perceived' or 'felt' stigma; 'self' or 'internalised' stigma; discriminatory or stigmatising practices by health services, legislation, media and educational materials; and experience by the affected person of actual discrimination and/or participation restrictions ${ }^{14}$. The assessment of health professionals' attitudes towards fat people and patients has been one of the most researched component of the stigma process in which many anti-fat attitude scales have been developed. These measures have been developed from diverse theoretical perspectives and designed to assess global attitudes relevant to the self ${ }^{15}$ (Crandall, 1994; Robinson, Bacon, \& O'Reilly, 1993) or the other person ${ }^{16}$ (Crandall, 1994; Greenwald, McGhee, \& Schwartz, 1998; Lewis et al., 1997), and to target specific populations and circumstances such as within healthcare settings ${ }^{17}$ (Bagley et al., 1989; Watson, Oberle, \& Deutscher, 2008). Due to the complexity of fatness, studies often incorporate more than one measurement scale into the research design or amalgamate subscales from multiple measurements. By doing so, it allows for the assessment of self, other, and practice (or intended behaviour) to be assessed simultaneously within one study.

Commonly used psychometric measurements specifically related to the assessment of weight bias attitudes include: Fat phobia scale (FPS) (Robinson et al., 1993), Implicit associations test-fat/thin (IAT) (Greenwald et al., 1998), Anti-fat attitudes questionnaire (AFA) (Crandall, 1994), Anti-fat attitudes test (AFAT) (Lewis et al., 1997), Attitudes towards obesity and obese patients (ATOOP) (Bagley et al., 1989), and Nurses attitudes towards obesity and obese patients (NATOOP) (Watson et al., 2008). The first four measurement scales are non-specific and can be applied to generic situations whereas the latter two measurements specifically target healthcare professional attitudes when engaging in patient care. It is important to note that this approach of reporting attitudes and practices alone does not necessarily accurately

13 Attitudes are "a set of beliefs, feelings, and assumptions that influence behaviour" (Zuzelo \& Seminaro, 2006, p.66).

${ }^{14}$ Participation restriction refers to the restriction of social participation in a wide range of every day experiences caused by actual discrimination or percieved stigma (Van Brakel, 2006)

${ }^{15}$ Measurements that assess the personal fear of becoming fat.

${ }^{16}$ Measurements that assess the attitudes towards other people who are fat.

17 Measurements that specifically assess attitudes of healthcare professionals towards providing care to fat patients. 
reflect the real situations encountered and requires a more expansive approach using qualitative methods to complement research in this area (Van Brakel, 2006).

There have been many assessment tools developed that assess the psychological impact of 'perceived' and 'enacted stigma' on individuals with chronic health conditions, although this area remains under researched. These tools generally assess levels of self-esteem, depression and similar constructs that affect a person's social participation. These type of tools are considered highly relevant to the assessment and monitoring of stigma reduction interventions, which not only assess and evaluate an individuals' social participation but also the effectiveness of programmes that promote social inclusion (Van Brakel, 2006). Currently, there have been no specific tools identified that measure social participation restrictions for fat individuals.

One limitation to this type of assessment is that restrictions to social participation can be greatly exaggerated by the person's disability, impaired activity or environmental factors which may not be easily differentiated from the effects of being stigmatised (Van Brakel et al., 2006). For example, fat people can have varying degrees of impaired mobility, may have significant disability, and be restricted in society and healthcare by the spacial arrangements and structural sizes of everyday furniture such as public transport seating (Forhan, Vrkljan \& MacDermid, 2010). This can significantly impact on their social participation, however all these factors may not necessarily be related to the construct of stigma.

There are very few instruments that assess 'enacted' stigma and actual discrimination; none of these are specific to measuring fat discrimination. More research on the assessment of actual discrimination is vital and two fold, given that many interventions target discrimination (Van Brakel, 2006). Firstly, this information would allow for the differentiation between stigma that is 'perceived' or based on 'actual' experiences which can lead to different approaches in how problems are tackled (Mental Health Commission, 1997). Secondly, it would aid in the evaluation of the impact that media campaigns, policy, and other forms of anti-discriminatory interventions may have on reducing stigma discrimination (Van Brakel, 2006). 


\subsubsection{Discrimination}

In its true sense the term 'to discriminate' means to distinguish between two options and therefore implies that a rational and deliberate choice has been made (Mental Health Commission, 1997). Thus, when exercising discrimination the individual or organisation has an interest in the outcome desired and is either for or against one of the options. If the effect of the chosen option is favourable then it is considered positive discrimination. For example, in 2008 the Ministry of Health made the decision to focus on improving nutrition, increasing physical activity and reducing fatness as one of its health $\operatorname{targets}^{18}$ (Ministry of Health, 2008b), in doing so, this rational and deliberate decision was favourable to those working in and requiring the particular services to improve general health and lifestyle. On the contrary, if the effect of the chosen option is restrictive in some form then it is considered to be negative discrimination (Mental Health Commission, 1997). For example, the decision by PHARMAC ${ }^{19}$ not to publicly fund certain cancer therapies (Parahi, 2014) may potentially lead to unfavourable outcomes for some cancer patients.

In today's society, the usage of the word 'discrimination' more commonly refers to the negative effects of the choices made and implies that a choice is made on an unfair basis to the detriment of the affected individual. It is this negative conceptualisation of the word which is used predominantly in sociological and health literature where the emphasis on unfair or negative behaviour is closely associated with the development of human rights legislation (Ministry of Justice, 1993). The Human Rights Act (1993) makes it illegal to negatively discriminate by unreasonably restricting or denying a person's ability to participate in society based on certain personal attributes, such as age, gender and race (Ministry of Justice, 1993). Discrimation in health research specifically focuses on certain stigmatised illnesses such as mental health illness (Mental Health Commission, 1997), HIV/AIDS (Siyam'kela, 2003), and fatness (Puhl \& Heuer, 2009).

${ }^{18}$ Health targets are a set of national performance measures specifically designed to improve the delivery of healthcare services and overall health of the population in identified priority health and disability areas (Ministry of Health, 2014).

${ }^{19}$ The Pharmaceutical Management Agency (PHARMAC) is an agency of the New Zealand Government that decides, on behalf of District Health Boards, which medicines and related products are subsidised within the healthcare system (Pharmaceutical Management Agency (PHARMAC), 2013). 
Within health research, discrimination is often said to have occurred "when stigmatisation is acted on by concrete behaviours such as exclusion, rejection, or devaluation" (Abbey et al., 2011, p. 2), and "when labelling and stereotyping leads to a loss of status that causes unequal outcomes" (Link \& Phelan, 2001). This can take place on a personal level or be enacted through societal and structural inequalities (Abbey et al., 2011; Link \& Phelan, 2001). There are three main types of discrimination with varying degrees of severity that lead to inequalities, which are direct, structural, and indirect discrimination (Link \& Phelan, 2006). Direct discrimination occurs when a person overtly rejects or excludes another person because of a personal attribute. For example, a physician who refuses the registration of a potential client to their general practice because they are fat is exercising direct discrimination. Structural discrimination is much more subtle and refers to social structures that are embedded in institutional practices, norms and behaviours that deny the rights and opportunuties of minority groups by preventing them from achieving the same opportunities as majority groups (Human Rights Commission, 2011). Structural discrimination can be both intentional and unintentional. An example of structural discrimination within healthcare would be the lack of access to medical and rehabilitation services, and inequalities in the quality of care that fat patients recieved. Lastly, indirect discrimination results from the process of 'internalised' stigma where stigmatised individuals are aware of the negative labels attached to them and respond in ways that exclude themselves from social participation (Link \& Phelan, 2006). An example of indirect discrimination would be the reluctance of fat patients to access healthcare services for fear of 'percieved' stigma.

Emerging theoretical work suggests that prejudicial attitudes alone are not sufficient evidence that a personal attribute such as fatness is stigmatising (Phelan \& Link, 2001). Moreover, stigma is evident when both the actions of the individuals and social institutions that degrade and exclude, occur in unison with the reactions of the person in the devalued social category (Link \& Phelan, 2001). If this holds true, this should be evidenced by the person in the devalued group being directly disadvantaged as a result of the behaviours of an individual or social institution. When applied to healthcare, the fat person would need to be directly disadvantaged through the care 
provided by healthcare professionals or by the organisational policies that govern the care provision of the healthcare facility. In this more expansive conceptualisation, it is uncertain whether fat people experience discrimination as a critical component of the stigma process (Carr \& Friedman, 2005; Link \& Phelan, 2001).

Additionally, the assumption that weight bias attitudes will be manifested into discriminatory behaviours is debatable, as there has been shown to be a weaker association between attitudes and actual behaviour than situational influences on actual behaviour (Eagley \& Chaiken, 1993). This disconnect between attitudes and behaviours has been suggested in other studies where implicit attitudes remained unchanged, despite healthcare professionals reporting positive professional and personal experiences working with fat patients (Schwartz et al., 2003). Despite prejudicial attitudes towards fat individuals being well documented (Brownell et al., 2005; Crandall, 1994; Lewis et al., 1997; Schwartz, Vartanian, Nosek, \& Brownell, 2006; Teachman et al., 2003), there is emerging evidence to suggest that these attitudes may not necessarily transpire into discriminatory behaviour (Eagly \& Chaiken, 1993). Few studies directly measure whether stigmatised groups are disadvantaged in life due to discrimination. Rather 'discrimination' is cited as a post hoc explanation when socially devalued groups demonstrate a shortfall in a life domain (Carr \& Friedman, 2005).

\subsubsection{Stigma, prejudice and discrimination in healthcare}

There have been numerous reviews of the empirical literature to determine if stigma, prejudice and discrimination of fat people exist in healthcare (Brown, 2006; Forhan \& Sala, 2013; Mold \& Forbes, 2013; Puhl \& Brownell, 2001; Puhl \& Heuer, 2009). Consistent findings across a number of studies have provided substantial evidence to confirm the presence of weight bias in healthcare. More specifically, that healthcare professionals endorse stereotypes and hold negative attitudes towards fat patients (Puhl \& Brownell, 2001; Puhl \& Heuer, 2009). However, the existence of this weight bias appears to be over represented in the literature due to the manner in which these minority findings, which approximate to $20-25 \%$ of attitudes, are used. Most noticeably these minority findings are used to formulate sociological arguments, perspectives and opinions in regards to the pervasiveness of weight bias in society as 
frequently reported by researchers such as Puhl and Brownell (2001, 2003), and Teachman and Brownell (2001). Researchers often neglect to provide comprehensive reports on the positive attitudinal findings of their research and what beliefs impact on these positive attitudes. Reasons for this biased reporting may be due to the limitations of methodologies used, measures used in assessing attitudes and beliefs, and the intention of the research to identify if bias, stigma, and discrimination exists in healthcare. Thus, there are significant issues in the way that others have drawn on the empirical data on stigma, prejudice and discrimination by favouring and reporting minority findings.

Additionally, the quantitative methods of examining weight bias may not accurately reflect individual attitudes (Fabricatore et al., 2005; Schwartz et al., 2003; Zuzelo \& Seminara, 2006). Quantitative study designs and the attitudinal measures currently used may be too limited in the diversity and scaling of attitudes and beliefs assessed, such as those that focus primarily on common stereotypes. Furthermore, these dated measures may no longer accurately capture todays common attitudes and beliefs thereby, forcing participants to respond to attitudes and beliefs thought to be prevalent 20-25 years ago. Therefore, it could be conceivable that these common stereotypes continue to be present and reported on because that is all that is being assessed. Establishing what are the new attitudes, beliefs and thinking about fatness requires these measures to be revised and revalidated in today's context particularly as the trend within the evidence suggests that nurses are less inclined to strongly agree with these common stereotypes (Culbertson \& Smolen, 1999), that were used in earlier studies involving healthcare professionals (Bagley et al., 1989, Maddox \& Liederman, 1969). What the qualitative studies presented in this chapter demonstrate is that these survey tools have limited a developing understanding of the context in which nurses engage in care, and that negative attitudes and beliefs do not appear to be at the forefront of influencing practices (Zuzelo \& Seminara, 2006). Instead, as with other patients, the physical care issues and safety of themselves and the patient appear as the most important issues.

There is adequate evidence to suggest that fat patients 'perceived' that there was bias in their healthcare treatment (Brown et al., 2006; Creel \& Tillman, 2011; 
Thomas et al., 2008), however additional research is needed to strengthen these findings (Puhl \& Heuer, 2009). Similarly, there is limited but emerging evidence to suggest that weight bias negatively impacted on healthcare utilisation or affected the weight management practices of providers (Drury \& Louis, 2002; Fontaine et al., 1998; Olson et al., 1994). However, no clear conclusions can be made from this emerging area of research (Puhl \& Heuer, 2009).

Based on the weak methodological approaches taken within the current evidence, discrimination, being that the fat patient had been disadvantaged in healthcare, could not be determined. No conclusions could be made as to whether fat patients were discriminated against, as inferences in the literature relied on selfreported perceptions of discrimination which were not directly measured in the studies (Puhl \& Brownell, 2001; Puhl \& Heuer, 2009). Therefore, the degree to which these negative attitudes and beliefs actually affected behaviours and practice could not be determined.

There is a general consensus within the literature that in order to advance research and knowledge of healthcare stigma, prejudice and discrimination there needs to be more diverse research methodologies (Brown, 2006; Puhl \& Brownell, 2001; Puhl \& Heuer, 2009). The use of experimental designs is required to assess the attitudes held by healthcare professionals towards fat patients, and identify how negative attitudes of healthcare professionals influence care practices (Puhl \& Brownell, 2001). Similarly, there needs to be more rigourous qualitative research that aims to improve understandings of attitudes and beliefs beyond common stereotypes with specific attention on how health concerns or physical demands of care affect different contexts, and how positive attitudes such as concern and empathy may ameliorate negative attitudes (Brown, 2006). Furthermore, there is a necessity to move beyond reports of attitudes to examining actual healthcare practices (Puhl \& Brownell, 2001) that focus on assessing care practices, behavioural expressions of weight bias, and physical settings of care delivery (Puhl \& Heuer, 2009). This review of the literature clearly identifies that more research is needed on actual behaviours of healthcare professionals that influence health outcomes and quality of care for fat patients. 


\subsection{Chapter summary}

This chapter has identified that the clinical research addressing the care of critically ill fat patients is scarce and medically orientated to focus on the management of fat specific physiological changes during periods of critical illness. Despite frequently cited concerns and consequences of caring for fat patients, the current intensive care literature provides little, if any, in-depth detail on the ways in which the size and shape of the fat body challenges everyday ICU care practice.

Although there is a large volume of literature on the social stigma of caring for fat patients, gaps have been identifed regarding the actual behaviours of healthcare professionals towards fat patients during care situations. The existing research is limited on the topic of weight bias and discrimination in healthcare, with evidence to suggest that these few studies have been used frequently to generalise that healthcare professionals have negative attitudes about fatness. What is known from the empirical data is that only a minority of nurses and healthcare professionals have negative attitudes and beliefs towards fatness and fat patients. Furthermore, there is evidence to suggest these attitudes and beliefs have changed over time, but what these shifts in ideologies and behaviours are have not been fully researched and are currently limited by the methodologies used. Additionally, the overall attitudes of nurses towards fat patients over the last 25 years have become substantially more positive. What is less known is how weight bias impacts on care practices, the quality of care delivered, and the utilisation of healthcare provision by fat patients, or how the attitudes and beliefs of the minority of healthcare professionals influence the behaviours of the dominant health workforce.

What is not known is whether discrimination exists in healthcare, as to date there have been no studies that directly measure discrimination by providing evidence that fat patients have been directly disadvantaged in healthcare as a result of the behaviour of an individual or social institution. Equally, it is not known whether healthcare professionals actually behave in a discriminating manner when caring for fat patients as observations of professional-patient interactions have not been undertaken in the context of weight bias, discrimination research. However, studies 
using self-reported, personal accounts of fat people's experiences of care within hospital would suggest that nurses do behave 'differently' towards fat patients; whether this leads to the patient being disadvantaged in healthcare has not been researched. Given the complexity of managing fat patients and the finite resources to appropriately care for this patient population, it could be debated that the situational influences of the healthcare setting plays a more significant role in the behaviour of healthcare professionals. As such the complexity of fatness and its management could potentially imitate itself as weight bias discrimination.

The nature of social interactions between healthcare staff and fat patients, beyond that which are self-reported, are not described in the literature. In healthcare, research mainly focuses on identifying the existence of stigma, prejudice, and discrimination via the assessment of self reported attitudes, leaving the nature and quality of interactions during actual care practices largely unexplored. In the next chapter, I examine the theoretical works of Erving Goffman $(1959,1967,1969)$ and Arlie Hochschild (1983) who have been influential in developing knowledge of social interaction and the management of emotions within the context of healthcare practices. 


\section{CHAPTER THREE: THEORETICAL INFLUENCES}

\subsection{Introduction}

The works of Erving Goffman $(1959,1967,1969)$ on the dramaturgical perspective of self in social interaction, and Arlie Hochschild's (1983) sociological theory of emotional labour have been instrumental in understanding how individuals interact with each other as part of everyday life. Although, these theories were developed outside of the healthcare setting, their theoretical conceptualisation of social interaction have considerable significance to healthcare practice and to the nature and quality of health professional-patient interactions during care delivery. These theoretical works have been used within this study to inform the conceptualisation of the care of fat patients as themes emerged from the interpretation of the data. In this chapter I examine their work in relation to social interaction and the management of emotions and provide a critique of its current application to the healthcare setting.

\subsection{Goffman's dramaturgical perspective of self in social interaction}

Goffman's seminal works on self, social interaction and social order have been instrumental in understanding 'everyday social life' (Branaman, 1997). This work has allowed for closer examination of how the self as a social product is presented to and received by others during social interactions. For Goffman (1959) interaction was considered to be "the reciprocal influence of individuals upon one another's actions when in one another's immediate physical presence" (p.26). Through the use of theatrical metaphors, Goffman viewed 'social life' as 'drama', 'ritual' and 'game' where individuals were considered to be performers, similar to actors on stage (Goffman, 1959, 1961a, 1967, 1969). Each metaphor intricately depicted a different element of social interaction. The dramaturgical perspective described by Goffman drew attention not only to the private and public dichotomy contained within social interaction and social behaviour but also the manipulative and moral aspects of social life (Branaman, 1997).

Goffman stressed that as social beings, individuals were concerned with living up to the many moral standards of society. However, as performers, individuals were 
"concerned not with the moral issue of realising these standards, but with the amoral issue of engineering a convincing impression that these standards are being realised" (Goffman, 1959, p. 243). This incongruent self, Fine and Manning (2000) termed as Goffman's "two selves thesis" (p.469). One self was the public performer giving carefully managed impressions while the second self was a cynical manipulator hidden behind the public performance.

Fundamental to Goffman's social theory was the idea that the self was a social product (Goffman, 1952, 1959, 1967). He described it as a product of the performances that the individual gave in all social situations where the sense of self arose from the public validation of these performances. Furthermore, the performances given by the individual were socially constrained presentations of self that were consistent with and supported by others within the social context in which it was performed. In other words, the self as a social product was dependent on validation that was awarded or withheld in accordance with the norms of that culture. It is assumed therefore, that in Goffman's concept of self an individual is always concerned about maintaining their own self-image based on the judgements by others of their performances. As such, individuals have many motives for trying to control the impression that others make of them in any situation (Goffman, 1959).

The concept of Goffman's dramaturgical perspective of drama, ritual and game have been used within healthcare to describe how nurses performed and segregated their work (Coombs, 2004; Lawton, 1998), behaved differently within the different spaces of their work (Cain, 2012; Tanner \& Timmons, 2000), and presented a professional self to others (Bolton, 2001; Cain, 2012). The following discussion is an exploration of how behavioural regions and face-work have been percieved, utilised, and critiqued within healthcare practice.

\subsection{Behavioural regions and healthcare}

Central to Goffman's (1959) dramaturgical metaphor of 'drama' in interaction was the concept of 'behavioural regions'. This concept was developed from the fieldwork conducted by Goffman in 1955 for 'The presentation of self in everyday life' where he observed hotel life in the Shetland Islands. He noticed that in the dining 
room kitchen staff and waiters behaved differently in front of the guests than they did when in the kitchen amongst colleagues. He described the dining room as the 'frontstage' where certain rules and roles must be acted out: "when one's activity occurs in the presence of other persons, some aspects of the activity are expressively accentuated and other aspects, which might discredit the fostered impression, are suppressed" (Goffman, 1959, p. 114). Front regions, such as the hotel dining room, were defined by the maintenance of proper appearances and adherence to rules of politeness and decorum ${ }^{20}$. The front contained the setting (furniture, props, physical layout) and the characteristics (age, gender, social status, ethnicity) of the individual performer. Additionally, the front existed separately from the person who occupied the role and therefore, when a person entered into the already established front they simply had to conform to the expectations of the social situation which assisted to reproduce the 'collective representation' of reality (Goffman, 1959). The need to conform to the rules, standards and expectations of the social situation meant that the front was highly scripted and rarely allowed for individuals to express their personal thoughts, feelings and desires. In this front region individuals were known to exaggerate their emotions for the audience in an effort to evoke particular feelings in others; a concept that Hochschild (1983) later termed emotional labour (see Section $3.5)$.

In contrast, the kitchen or 'backstage' region was observed to be a place of contradictory rules where suppressed facts made an appearance. The backstage was seen as "a place, relative to the given performance, where the impression fostered by the performance is knowingly contradicted as a matter of course" (Goffman, 1959, p. 114). The back regions were places that were out of sight from the public, but usually next to the place where performances occurred, and contained personal items of the performers that were not needed or out of character for the staged front show. The backstage was a place where performers could momentarily step out of character and relax in the knowledge that no member of the audience would intrude. Backstage behaviour and language was frequently informal, unstructured and often considered

20 'Politeness' referred specifically to the standards the perfomer must maintain while engaged in conversation. Whereas 'decorum' referred to standards that must be maintained in situations where the performer was in visual or aural range of the audience but not necessarily enaged in talk with them (Goffman, 1959). 
potentially offensive if it were to be performed on the frontstage. Backstage language consisted of:

Reciprocal name-calling, co-operative decision-making, profanity, open sexual remarks, elaborate griping, smoking, rough informal dress, 'sloppy' sitting and standing posture, use of dialect or sub-standard speech, mumbling and shouting, playful aggressivity and 'kidding', inconsiderateness for the other in minor but potentially symbolic acts, minor physical self-involvement such as humming, chewing, nibbling, belching and flatulence (Goffman, 1959, p. 129).

In contrast, frontstage behaviour was considered to be the absence or the opposite of backstage behaviour where offensive language was disallowed. Performers moved between these two regions and adjusted their language and behaviour accordingly. Goffman (1959) claimed the back region behaviour had to exist in order to make the front behaviour possible. Backstage allowed performers time and space to prepare for their roles, practice appropriate ways of presenting themselves, release any tension or frustration, and take a break from the emotionally tiring roles. As a result the behaviour of the backstage kept the frontstage in order.

Regulation of access between the different regions was vital to maintaining the performance. Each area of the stage was clearly demarcated so individuals knew where they were allowed to be. Performers, like the hotel staff, appeared in both the front and back regions whereas the audience, the hotel guests, were only permitted to be in the front region. Outsiders, who were neither staff or paying guests, were not permitted at all in any region. A glimpse of the back region by the audience or outsider could destroy the whole performance: "The audience must not acquire destructive information about the situation that is being defined for them...a team must be able to keep its secrets and have its secrets kept" (Goffman, 1959, p. 141). Information control between the front and back regions was at the centre of managing performances and convincing others of the fostered impression of reality.

Behavioural regions has been used to describe and emphasise the power and hierarchical structures that exist in healthcare (Coombs, 2004; Tanner \& Timmons, 2000), conceal the realities of death and dying from others (Lawton, 1998, 2000; Prior, 
1987), and construct professional identities that supported the emotional aspects of nursing work (Cain, 2012). The nature of clinical decision-making in the intensive care setting was explored using Goffman's theatrical metaphor as an analytical framework (Coombs, 2004). Decision-making, Coombs' (2004) proposed, was both formal and informal, staged in official and unofficial regions of the unit/hospital, and was full of dramatic licence. Formal decision-making occurred within public and formalised environments such as ward rounds and service (operational and business) meetings, where roles were rehearsed and well scripted. These forums occurred in the frontstage regions where different social roles were acted and performed in front of their audience of peers, team members, patients and families. The frontstage was a "high profile setting" (Coombs, 2004, p.106) where decision-making was most visible and undertaken by the most senior and authoritative roles who presented carefully scripted performances. This high profile region was "centre stage" (Coombs, 2004, p. 106) for the most public and dramatic forms of decision-making that involved life and death decisions for the patient.

In accordance with Goffman's backstage, Coombs (2004) regarded the backstage region as a place where doctors and nurses, hidden from public viewing, could rehearse and prepare for their frontstage requirements of patient care. It was here that staff shared information about patient management and identified current problems that would inform frontstage decisions. Thus, backstage informal one-onone discussions, and nursing and medical handovers provided the script that would be carefully delivered on the frontstage. The work that occurred in the backstage was deemed vital to ensuring the frontstage ran efficiently.

In Coombs' (2004) work she proposed that there were unofficial behavioural regions of the stage that ran parrallel to and supported the official front and backstage clinical decision-making performances. Often these unofficial regions were unrecognised and therefore not acknowledged within the decision-making process. The unofficial frontstage was a place where decision-making activities occurred in public by individuals "not normally considered to have this particular script or role" (Coombs, 2004, p. 106). These decisions went unnoticed by others because of the lack of an official decision-making forum but nevertheless were a crucial aspect of the 
overall clinical decision-making for the patient's management. Although, Coombs (2004) identified that there was an unofficial backstage, which would have consisted of private coffee room discussions, and an offstage region of lay world public meetings, these were not explored in her study.

Through the use of the official and unofficial stages Coombs (2004) was able to draw attention to the ways in which roles and knowledge produced power and conflict in interdependent decision-making. The official frontstage was dominated by biomedical knowledge and financial frameworks for decision-making, the recognised authority of medicine to make decisions, and the surveillance work performed by medicine and nursing. Thus, the frontstage of decision-making was dominated by and belonged to medicine. On the official frontstage, nurses did not have a high profile and their contribution to decision-making was invisible and not recognised by their medical colleagues, managers or indeed nursing colleagues.

In contrast, nurses operated predominantly on the official backstage and unofficial frontstage. The official backstage was dominated by knowledge and management of the environment and resources by nurses, where the role adopted was that of "acting as the organisational glue for the system" (Coombs, 2004, p. 109). By this, Coombs suggested the knowledge, skills and co-ordination of resources and services were often invisible work in the decision-making process but nonetheless an essential component. Similarly, nurses dominated the unofficial frontstage where their experience of the environment, and knowledge, from continuity, was used to influence decision-making. This was a place where the nursing role focused on working flexibly with the decisions made to ensure the whole system would "function efficiently and not become overloaded with demands for decision and action" (Coombs, 2004, p. 109). Thus, the main contribution nursing made to decisionmaking "was not located in the powerful, public frontstage" (Coombs, 2004, pp.109110).

The examination of operating theatres as a backstage region of the hospital has been used by Tanner and Timmons (2000) to provide new insights into the behaviours, blurring of professional roles, and power structures of theatre staff. Whilst Tanner and 
Timmons (2000) acknowledged that the operating theatre, for a brief time, was a frontstage region (while the patient was waiting to be anaesthetised), it was predominantly considered a backstage region; the latter being the focus of their research. In the 'private' environment of the operating theatre Tanner and Timmons (2000) noticed that there were marked behaviour changes, unprecedented familiarity and platonic physical closeness in the interactions between different groups of staff, that was not evident elsewhere in the hospital. The boundaries of professional roles were blurred as surgeons assisted nurses with moving theatre equipment, and transferring patients from beds to operating tables, and finding the correct type of suturing material for the nurses. Similarly, nurses took more medical and surgical assistant roles which involved direct involvement with the surgical procedure.

Despite this more informal relaxed social interaction between medicine and nursing, traditional hierarchical structures of the doctor-nurse relationship existed. Nurses were conscious of the professional role hierarchies and acted in ways that acknowledged this structure by always stepping out of the way for the surgeon and seeking approval from the surgeon despite having already made a decision. Conversations between different groups reflected the hierarchial status of doctors and nurses. Doctors were observed to have frequent serious and intellectual discussions with each other, whereas conversations between doctors and nurses were often trivial and amusing. Similarly, once outside of the operating theatre the relationship between doctors and nurses reverted back to that which reflected the rest of the hospital where surgeons would walk past theatre nurses and not speak to them. In Tanner and Timmon's (2000) work nurses appeared to have a false sense of their social position as they mistakenly took "the surgeon's backstage behaviour to signify changes in the balance of power and hierarchy in theatre" (p.978).

Concealing the realities of death and dying from others has been explored by examining the different 'stages' on which death is managed. Lawton's (1998) work on 'dirty dying' has provided insight into how particular ways of dying were confined to certain stages. 'Dirty dying', Lawton $(1998,2000)$ suggested, was a backroom activity due to the need to conceal the disintegrating, leaking and decaying body from others. This she described as the "unbounded" (Lawton, 2000, p.134) body which 
needed sequestrating from other 'bounded' dying patients due to the percieved malodorous, ugliness, and dirtiness of dying. Furthermore, 'dirty dying' was contained within the confined space of the hospice separated from the outside world, where access was restricted to close family and friends. The importance of this backroom activity meant that 'bounded' patients and the outside world could be protected from the realities of the dying process. In doing so, the frontstage illusion of peaceful, painfree deaths could be maintained.

Managing the impression of nice and peaceful deaths was explored in Prior's (1987) work on the preparation of bodies in the post-mortem room. The activities performed on the dead body by undertakers were confined to the privacy of the 'backstage' post-mortem room. This place was highly restricted so as not to reveal the secrets of this backstage activity that would disrupt the "illusion that the dead one is really in a deep and transquil sleep" (Goffman, 1959, p. 116). The mortuary room was considered the place where bodies were prepared for their final performance on the 'frontstage' chapel of rest.

Worker identities were examined by Cain (2012) to understand how the decrepanies in front and backstage behaviours of hospice nurses created a hospice identity. She challenged the assumptions that backstage performances were more authentic than frontstage by demontrating how hospice workers integrated both regions into a professional sense of self. She proposed that the two regions had a "symbiotic relationship" (Cain, 2012, p. 669) whereby backstage activities allowed for appropriate frontstage behaviour while frontstage activities provided material for backstage conversations. In this symbiotic relationship hospice workers presented themselves in the front region as "caring, compassionate and serious professionals" (Cain, 2012, p. 669), while in the back region workers' behaviour consisted of dark humour, strategising to change patient and caregiver conduct, and exhibiting detachment from death. These juxtaposed behaviours were considered both to be authentic expressions of self necessary to maintain professional identity, and as a means for managing the stresses of emotional labour (see Section 3.5). 
For Cain (2012) the frontstage professional identity was considered to be one that supported the hospice philosophy that encouraged workers to make real connections with patients and their family. Equally, the ability to feel real authentic emotions that did not detract from the anguish of the families was an important component of this professional identity. The backstage professional identity was considered to be one of "enlightenment about death" (Cain, 2012, p. 686) where hospice workers understood the inevitability of death. Their behaviours reflected this acceptance of this impending death as exhibited by distancing themselves, making light of the situation, and preparing dialogues to educate patients and caregivers about managing the terminal processes of dying. Cain (2012) proposed that because hospice workers were able to integrate both sets of behaviours and professional identities into their work life and sense of self, they were better able to perform their work and continue to provide care, especially under stress.

\subsection{Face, face-work and healthcare}

Central to Goffman's (1967) dramaturgical metaphor of 'ritual' interaction is the concept of 'face' and 'face-work'. Goffman defined 'face' as 'the positive social value a person effectively claims for himself by the line others assume he has taken during a particular contact" (Goffman, 1967, p. 5). During every encounter a person must act out what Goffman referred to as 'a line', which was a pattern of verbal and non-verbal behaviours that expressed his view of the situation through his appraisal of both himself and other (Goffman, 1967). He suggested that a person's face "is not lodged in or on his body but rather something that is diffusely located in the flow of events in an encounter" (Goffman, 1967, p.7). This social face could only be made meaningful by particular contexts and the sequences of interactions in which it occurred. For example, how a smile was received and interpreted had different meanings in different contexts and between different people.

A person's social face was considered to be his "most personal possession and the centre of his security and pleasure" (Goffman, 1967, p. 10). However, because of the nature of social interactions the social face was only ever loaned to him by society and could be withdrawn from him if he did not conduct himself in a manner worthy of 
it (Goffman, 1967). Goffman's social construction of face was therefore, both socially and psychologically vunerable during encounters with others.

Goffman (1967) proposed that the social face was directly connected to a person's emotional well-being as it could be lost, maintained or enhanced during social interactions. A person that was "in face" (Goffman, 1967, p. 6) felt emotionally secure and confident and could present himself to others in the knowledge that the line he was taking was supported by others. In contrast, when a person was "in wrong face" or "out of face" (Goffman, 1967, p. 8) he felt emotionally inferior, ashamed and embarrassed because of how his reputation would be affected by the encounter which threatened his image of self. Individuals managed this social vunerability during social interaction through face-work.

Goffman's (1967) face-work describes a theory of ritual interaction in humanto-human encounters whereby individuals interpret and act accordingly to maintain the face of self and other. During the interaction an individual presents to the other person a particular portrayal of how the self is to be evaluated. Primarily, the purpose of face-work is to manage the impressions, or face, of both self and other. To do this, a person must simultaneously engage in defensive and protective strategies aimed at saving one's own face whilst preventing the other person from losing face (Goffman, 1967).

People engage in different types of face-work on a regular basis in order to protect their own face and that of others. How individuals perform face-saving acts often becomes habitual and standardised like traditional dancing steps. As such, each person, subculture and society has its very own repertoire of face-saving practices which likens the interactions to that of what a person or culture is really like (Goffman, 1967). Types of face-work include riutals, corrective process, avoidance process and poise. Rituals are routine behaviours expected to be enacted by individuals in every encounter such as ritual greetings and closings of encounters. Politeness is an expectation of this ritual. Corrective processes are needed by a person who causes another to be viewed negatively or feel insulted by their direct or indirect actions. Often these events are considered to be 'incidents' that cannot be overlooked 
and demand immediate attention. The offending person must engage in a corrective process of repair which usually involves an offer of apology. For the corrective process to be successful and the face of both individuals reinstated to one of positive social value, the defended person must accept the apology. The avoidance process is a defensive strategy used by individuals to either avoid contact in which threats to face will occur or minimise threats once engaged in an encounter. In the latter, the person deliberately avoids topics of conversation that would expose information that is inconsistent with 'the line' he is maintaining or redirects the conversation to another topic at the earliest opportunity (Goffman, 1967).

When a person's face has been threatened, one is expected to control the embarrassment experienced by the incident, by exhibiting poise. The term poise within this context of social interaction refers "to the capacity to suppress and conceal any tendency to become shamefaced during encounters with other" (Goffman, 1967, p. 9). It is expected in this situtation that others are to ignore the embarrassing incident or dismiss it as unimportant thus, supporting the other person to save face. Sometimes the only way to prevent a threat to face is to overlook an event through engaging in 'tactful blindness.' This is where a person acts as if the event that contained the threat did not occur at all. These type of threats are specific in nature, in that, the event could not be considered anything other than face threatening. Examples of face threatening acts that require another to tactfully ignore the behaviour or event include primarily those that involve involuntary loss or movements of the body such as flatulence, facial twitches, and stutterings (Goffman, 1967). In nursing, tactful blindness is commonly employed during the care of unwell patients who have become dependent of nursing staff to assist with hygiene and toileting needs.

Goffman's (1967) notion of face and face-work, has been extensively developed and incorporated into theoretical models of embarrassment (Cupach, Metts, \& Hazleton, 1986), and politeness (Brown \& Levinson, 1987; Lim \& Bowers, 1991), applied to understanding nurse-patient interactions (Meerabeau, 1999; Shattell, 2002, 2004; Spiers, 1998), changes in social relationships following disgnosis and treatment of disease (Little, Jordens, Paul, Sayers, \& Sriskandarajah, 2000), and contradictory practices and demands of nursing work (Bolton, 2001; Shattell, 2002). 
Embarrassment models have extended Goffman's (1967) face-work beyond his expectation that individuals must exhibit poise, by recognising that there were times when further loss of face could occur during embarrassing situations when poise was lost (Cupach et al., 1986). The model developed by Cupach et al. (1986) specifically addressed strategies that restored social interaction produced by "loss of poise" (p.183) and "improper identity" (p.184) ${ }^{21}$. Loss of poise was considered to reflect a person's ineptitude within a social interaction whereas improper identity had greater negative implications for other persons who were present (Cupach et al., 1986). Impression management strategies used to restore the person's face following an embarrassing situation were apologising, accounting, and avoiding. Restoring face caused by loss of poise employed avoidance strategies that attempted to deny or minimise attention. Whereas, restoring one's public image as a result of an improper identity utilised strategies of apology and accounting for the unanticipated embarrassment by either justifying the behaviour or making excuses (Cupach et al., 1986).

The construction of Brown and Levinson's (1987) politeness theory ${ }^{22}$ has expanded on and reconceptualised Goffman's face and face-work. Most notably, their notion of 'face' departed radically from that of Goffman's as, at the centre of their model, a dualistic notion of face existed with matching positive and negative politeness behaviours (Bargiela-Chiappini, 2003). The 'positive face' was aimed at securing that one is liked and respected and associated with the basic human needs for esteem and need for control. Whereas, a 'negative face' desired to be free from constraint and imposition and was associated with automony, territorality, and independence in thought and action (Brown \& Levinson, 1987). Threats to "positive face' occurred through expressions of strong negative emotions, criticism, disapproval, discussing taboo topics, and blatant non-cooperation in activities. Threats to 'negative face' occurred through orders, requests, advice, reminders, threats, or warnings that limited automony (Brown \& Levinson, 1987).

\footnotetext{
21 'Improper identity' refers to an identity that does not fit the social position held. For example, a restaurant customer who has misplaced his wallet cannot assume the correct social identity of a 'paying customer.' This improper identity therefore causes embarrasment (Cupach et al., 1986).

${ }^{22}$ Brown and Levinson (1987) develop a linguistic politeness theory to explain how language use in social interactions maintained 'polite friendliness' and 'polite formality.'
} 
Similarly, Lim and Bowers (1991) expanded further on both Goffman's (1967) and Brown and Levinson's (1987) concepts of 'face' and politeness theory by describing three forms of 'face' and face-work activities. Face-work activities of solidarity, approbation and tact were used to support and maintain the 'fellowship face', 'competence face' and 'autonomy face' during polite interaction. Solidarity referred to strategies orientated towards fulfilling the 'fellowship face' (or Brown's and Levinson's (1987) 'positive face') needs of fellowship and belonging. Approbation addressed 'competence face' needs where appreciation of the other person's abilities was expressed by minimising blame and maximising praise and compliments. Tact referred to avoidance strategies to support the 'automony face' by demonstrating respect for the other person's liberty and autonomy by enabling choice and limiting overly directive requests. The 'autonomy face' was akin to Brown and Levinson's (1987) 'negative face'. Lim and Bowers (1991) identified that relational intimacy was the strongest predictor that face-work would occur in an interaction. Furthermore, face-work was found less in situations where a person had power and the endorsed right to act in certain ways (Lim \& Bowers, 1991).

Face and face-work has been used to describe how communication is negotiated and mutually constructed in nurse-patient interactions (Shattell, 2004; Spiers, 1998). Spiers (1998) proposed that the application of face-work theory provided new ways of understanding the context of the nurse-patient interaction, and the actual patterns of speech based on the perceptions of 'face' presentations, and the inherent structures of power, culture, and social distance. This depth of understanding of how communication was directed by cultural and basic human needs, ${ }^{23}$ Spiers (1998) argued, was lacking in nursing communication frameworks. These frameworks primarily focused on strategies "presented as a list of skills that ...facilitate[d] the development of a therapeutic relationship" (Spiers, 1998, p. 27).

Spiers (1998) applied face-work models of politeness (Brown \& Levinson, 1987; Lim and Bowers, 1991), forms of address (Wood \& Kroger, 1991), and

\footnotetext{
${ }^{23}$ Basic human needs in this context of social interaction refer to autonomy, competency, selfesteem, and belongingness (Spiers, 1998).
} 
embarrassment (Cupach et al., 1986), to understanding nurse-patient communication. Spiers (1998) identified that nurse-patient communications were mutually negotiated, responsive to the demands of the situations, and attended to the interpersonal needs of both the nurse and patient. The incorporation of face-work theory into nursing communication Spiers (1998) proposed, would greatly enhance understandings of how nurses and patients react when their autonomy, dignity and poise are threatened, and how situations of intrusion, loss of poise and embarrasment are mutually managed.

Goffman's face-work theory has been applied to the existing literature on nurse-patient interactions (Shattell, 2004). The review of the literature specifically focused on four aspects of the nurse-patient interaction: nurse communication, the actual interaction, patient perceptions, and patient care-seeking communication. Shattell (2004) identified that the quality of nurse-patient interactions were significantly effected by issues of power, social and cultural contexts, and the interpersonal competence of the nurse and patient. Threats to face appeared greater in situations of patient vunerability, such as being asked about intimate personal matters, or when illness resulted in dependency on nurses for fundamental care needs. In these nurse-patient interactions, the potential for the patient to lose face (autonomy and selfesteem) was considered to be extremely high.

The examination of illness narratives of patients with colorectal cancer provided insight into how patients used face-work to manage the loss of 'face' and recreate new 'faces' that conveyed a new sense of identity (Little et al., 2000). From these illness narratives Little et al. (2000) proposed a model of face whereby the 'face' was integral to honour and dignity. Honour, Little et al. (2000) proposed, was derived from a person's social positioning in relation to the systems in which s/he moved, whereas dignity expressed the personal attributes and acquisitions of the person. Both honour and dignity, to differing extents, were simultaneously affected by the loss of 'face'.

A patient's sense of personal honour and dignity prior to their diagnosis and treatment was identified as being central to the loss of 'face' they encountered 
throughout their illness and then how well it could be successfully compensated for (Little et al., 2000). Patients' who identified that their sense of identity, 'face' and honour were significantly attached to their social status, power and reputation, reported loss of 'face' through feelings of dishonourment when they experienced the levelling effects of the healthcare system. The person's ability to manage this threat to 'face' directly exposed his expressions of dignity. Likewise, patients' who identified that their sense of identity, 'face' and dignity were related to their physical attributes of looks, physique and health reported loss of 'face' through feeling undignified when coping with interactions that exposed the physical aspects of their illness, such as managing their stoma bags. ${ }^{24}$ The way individuals responded to threats and actual loss of 'face' was identified to be dependent on their "genetic attributes, the acquisitions that elaborate and express those attributes, and the capabilities that individuals express within the social system" (Little et al., 2000, p. 241). In other words, how a person was able to reconstruct their 'face' and sense of identity was very much dependent on the varying proportions of honour and dignity lost and reclaimed within their illness experience.

The many 'faces' of nurses and how they were used to manage the emotional demands made of them was identified in the work of Bolton (2001). In this work Bolton (2001) used Goffman's (1959) 'presentation of self' to understand the emotion work $^{25}$ of nurses working in a National Health Service Trust hospital. Bolton (2001) noticed that nurses managed the contradictions between their professional values and those of managerialism and economic markets through the presentation of different 'faces' during everyday working practices. These faces were 'professional', 'smiley' and 'humourous'.

The 'professional face' was considered to have contradictory elements in that nurses presented themselves as "caring whilst remaining distant" (Bolton, 2001, p.90). Nurses engaged in strategies of detachment and aloofness as ways of protecting themselves from revealing personal feelings that could make them lose 'face'. The loss of 'face' was considered to alter the power relationship between them and their

\footnotetext{
${ }^{24}$ Stomas are surgically created opening in the intestines which allows for feaces to bypass the rectum and be collected in a bag attached to the outside of the abdomen (Colwell, 2004).

${ }^{25}$ Emotion work (see Section 3.5).
} 
patients and suggested to others that they were no longer in control of their nursing work. When patients demonstrated total disregard for the rules of the ward, nurses often used their professional 'face' to mask their feelings of anger, which if displayed could make them lose 'face' for not acting in accordance with the professional values of nursing.

Nurses in Bolton's (2001) work used the terms 'face', 'mask', and 'act' when describing their professional role signifying their awareness of how they worked through their emotions in order to present the expected 'face' of a professional nurse. However, this 'professional face' the nurses reported was not always possible as often there were days that they felt emotionally exhausted. During these times nurses presented 'detached' or 'hard' faced performances which they recognised were unsatisfactory but all they could offer. Bolton (2001) observed that nurses were at times unsuccessful in altering their demeanor when presenting their 'detached' face as they could not conceal their feelings of exhaustion, demoralisation, sorrow or anger. These outward expressions of 'face' observers described as "hard-faced, aggressive or sulky" (Bolton, 2001, p.92).

The 'smiley face' was supported by completely different motives than those of the 'professional face' as it did not contain any elements of caring. The 'smiley face' was considered the customer service 'face' of healthcare that was required of nurses since the reinvention of patients as 'customers' and consumers of healthcare. Nurse were expected to present a 'smiley face' as part of their nursing work "in order to produce the marketable product of consumer satisfaction" (Bolton, 2001, p.93). The 'smiley face' focused on "keeping up appearance" (p.93), delivering high quality services, and acting in ways that would avoid consumer complaints. This customer service focus of managing the ward, nurses felt detracted from the real work of caring which at times meant being assertive with patients and dealing with situations more firmly for the health benefits of the patient. However, with customer satisfaction high on the health service agenda nurses revealed that they had to work harder on managing their emotions to avoid future complaints. Bolton's (2001) customer service focused 'smiley face' shares similarities with Hochchild's (1983) work on 
observations of flight attendants where the 'smiley face' that was presented to passengers represented the corporate interests of the organisation.

The 'humourous face' was considered not to be controlled by the rules of the healthcare organisation or nursing profession. The 'humourous face' presented itself when nurses were able to find spaces where they did not have to maintain the 'professional' or 'smiley face'. The 'humourous face' was used to maintain collegial connections, relieve frustration and anxiety, or to acknowledge resistance to demands made by management. 'Humourous faces' were often brief where a "shared smile, an exaggerated sigh, or a sideways glance” (p. 95) spoke volumes about the way a nurse was feeling about the situation they were involved in. Thus, 'humourous face' was not confined to the backstage but was present amidst the "organisationally prescribed performance” (p. 95). Bolton (2001) likened these instances to Goffman's “double stance" whereby actors were able to simultaneously adhere to the "official definition of the situation" while showing that he had "not agreed to having himself defined by what [wa]s officially in progress" (Goffman, 1961b, p. 133). Both Bolton's (2001) and Goffman's (1961b) interpretation and use of 'face' suggests that more than one face can be presented at the same time. Through the use of many 'faces' nurses were considered to be emotional jugglers with a capacity to present a variety of 'faces' and calibrate their performances according to the situations confronting them.

\subsection{Hochschild's concept of emotional labour}

Hochschild's (1983) seminal work on the concept of emotional labour examined the work undertaken by flight attendants. She demonstrated that in conjunction with the physical aspects of work such as working in confined spaces, serving refreshments, and acting quickly and safely in emergency situations, a substantial part of the job was attending to the emotions of passengers. During interactions with passengers flight attendants were required to produce positive emotional responses in their customers by always appearing to be 'nice' and 'enthusiastic'. These emotions displayed by flight attendants were subject to the control of their employer and were managed primarily to serve the financial interests of the airline company. Workers were expected to pay 'special attention' to those who brought "in the most money" (p.138) even if they did not "feel like being nice" to 
them (p.138). Hochschild (1983) identified that the passenger's ticket represented "the right to unsuppressed anger at irritations, having purchased that tacit right" (p.110). Thus, being nice to obnoxious passengers and hiding feelings of anger and frustration, was a mandatory requirement of the job. Every act of service, such as a smile, or pleasantry was viewed as an advertisement for the company. Passengers judged the quality of the service by the emotional style in which that service was given. Feelings were therefore considered to be a commodity that had an exchange value which attracted economic rewards.

From these observations of flight attendants work, Hochschild (1983) conceptualised emotional labour to be the trained and paid management of feelings that required the worker "to induce or suppress feelings in order to sustain the outward countenance that produces the proper state of mind in others" (p.7). In other words, emotional labour was the commercialised exploitation of feelings to create a sense in others of being cared for in a convivial safe place. The three fundamental characteristics of emotional labour were defined as face-to-face or voice-to-voice contact with the public, a requirement that the worker produce an emotional state in another, and through the training and supervision the employer was able to regulate a degree of control over the worker's expressed emotions (Hochschild, 1983; Smith, 1992).

Drawing on the work of Goffman's dramaturgical perspective (1959) and face-work (1967), Hochschild proposed that flight attendants worked to separate their private feelings from their commercially expected public displays of emotion. To do this, worker's had to engage in 'emotion work' which Hochschild (1979) defined as the "act of trying to change in degree or quality an emotion or feeling... [and] refers to the effort... and not to the outcome, which may or may not be successful" (p.561).

There were fundamental distinctions made by Hochschild (1979, 1983) between emotional labour and emotion work (emotion management). Primarily, emotional labour was a waged and therefore, commercialised commodity that had exchange value thus creating economic gains. Whereas, emotion work was private, unwaged and restricted to familial interactions and held a use-value. When emotion 
work was not confined to and used in private but entered into the marketplace, Hochschild (1983) claimed it transformed into an exchange value and was sold for a wage in the form of emotional labour. Thus, both emotion work and emotional labour undergo the same processes to achieve the desired emotional responses and displays in another person but the value pertained within that interaction served different sociological and economical purposes.

To achieve the desired emotional displays expected by their employers when how they naturally felt did not fit with the company's public image, employees engaged in method acting (Ashforth \& Humphrey, 1993; Diefendorff \& Gosserand, 2003; Hochschild, 1979, 1983). 'Surface acting' was one method where workers deceived others about what they really felt by suppressing their true feelings and pretending to feel what they did not in order to create a positive emotional response in the other person. In doing so, individuals faked unfelt emotions whilst suppressing naturally felt emotions. In contrast, 'deep-acting', as a means to display the expected appropriate emotions, required the individual to work on changing feelings within themselves by "trying to feel what we sense we ought to feel" (Hochschild, 1983, p. 42). This type of method acting allowed individuals to believe in the emotion being expressed giving it a more authentic exchange. In this instance, individuals modified felt emotions so that genuine displays of emotion can occur: "the actor does not try to seem happy or sad but rather expresses spontaneously...a real feeling that has been self-induced" (Hochschild, 1983, p. 35).

The emotional responses that could be displayed within certain interactions and situations were governed by 'feeling rules' which were defined as "guidelines for the assessment of fits and misfits between feeling and situation" (Hochschild, 1979, p. 566). For flight attendants, feeling rules were a set of commercially developed rules which would maintain a positive experience for the customer. These rules meant that non-conforming emotional responses of the flight attendants, such as frustration and annoyance, were suppressed and replaced with appropriate commercial emotions of niceness and enthusiasm, using surface and deep acting techniques. 
The demands of emotional labour involved in the work of flight attendants came at a cost to its workers. Hochschild (1983) found that suppressing feelings and putting on a 'commercial show' led to job dissatisfaction, emotional numbness and burnout. The perceived non-reciprocal nature of the work by flight attendants led to Hochschild identifying emotional labour as both unrewarding and a burden.

The concept of emotional labour has been used widely within healthcare as a way of describing how feelings and emotional responses are managed by nurses in their work. Despite the clear distinctions made in Hochschild's work between emotional labour and emotion work, these concepts are often used interchangeably within sociological and nursing literature (Gray \& Smith, 2009; Zapf, 2002). Consequently, McClure and Murphy (2007) have argued that this interchangeability has led to misunderstandings and misuse of emotional labour and emotion work in the nursing literature, thus, hindering its theoretical and empirical use in professional nursing theory, practice and research. The following discussion is an exploration of how emotional labour has been perceived, utilised, and critiqued within nursing practice.

\subsection{Emotional labour: An aspect of caring work}

A review of the nursing literature revealed that emotional labour has been frequently used to describe and highlight the undervalued tacit caring work of nurses, which was both gendered and invisible in nature (Gray, 2009; James, 1989, 1992; Smith 1991, 1992; Staden, 1998). Furthermore, it has created a means for nursing scholars to "grapple with the conceptual complexity of defining care, especially in relation to its emotional components and demands" (Smith, 1992, p. 9). For this reason emotional labour in nursing often takes on a meaning different to that intended by Hochschild (1983), which predominately focused on managing the emotions (often negative in nature) that did not fit the company's public image and would potentially jeopardise commercial revenue.

The invisibility of nursing work and its direct links to gender were identified in the work of James (1989) who used Hochchild's (1983) conceptualisation of emotional labour to understand the work of nursing the terminally ill. James (1989) 
saw the similarities in the work undertaken by nurses to that of unpaid domestic labour. She equated the invisibility of emotional labour carried out by hospice staff to the emotion work done by females within the family home. James (1989) argued that emotional labour relied heavily on the women's innate caring role in the private sphere of home by demonstrating how women's emotion management skills were brought from the private domestic domain into the public sphere of nursing practice. Thus, James' (1989) work challenged the dichotomous nature of emotion work and emotional labour by demonstrating that the clear division between the private and professional spheres of the nurse could not be so easily delineated as suggested in Hochchild's (1983) original work.

The dichotomy of emotional labour and emotion work has been substantially criticised in the nursing literature for its overly simplistic perspective that emotion work was purely private acts contained to the family home (Bolton, 2000; Callahan \& McCollum, 2002; Staden, 1998). Bolton (2000) argued that emotional labour, as defined by Hochschild (1983), was not enough to sufficiently explain the emotionally complex demands made of professional nurses. From her work observing gynaecology nurses involved in supporting women through miscarriage and foetal terminations, Bolton (2000) claimed that emotional labour was in fact emotion work which nurses offered to their patients as a gift. She observed that whilst nurses subscribed to professional feelings rules (i.e emotional labour), they also, at their own discretion, chose to freely add something extra to the nurse-patient relationship through emotion work.

Similar to James' (1989) gendered positioning of emotional labour in nursing, Smith and Gray (2001b) identified that caring relationships in the public domain tended to be percieved as part and parcel of a stereotype of women's private role in the domestic domain. For this reason, emotional labour was seen to be an essential nursing skill that did not require development or professional training because those who chose to nurse were intrinsically caring and nuturing (Smith, 1992). The assumption that emotional labour was an innate skill was questioned by Smith through her application of the concept to nurse education and ward environments (Smith, 1991, 1992; Smith \& Gray, 2001a, 2001b). She identified that emotional 
labour needed to be taught and had been previously embedded into the role of the ward sisters who were in charge of providing clinical knowledge and developing the interpersonal skills of the student nurse in learning how to care for patients. Following the change from hospital to tertiary provider nurse training, Smith focused her later research on exploring emotional labour in the context of developing new ways of learning to care for patients (Smith \& Gray, 2001a, 2001b). Learning to care, Smith and Gray (2001a) proposed, involved learning clinical and emotional skills which they referred to as "craft knowledge" (p.48).

The research conducted by Smith $(1991,1992)$ focused on analysing student nurses' accounts of their experiences of caring for patients when working within the ward team. Smith (1992) suggested that the descriptions of care by student nurses which included learning how to manage the emotions of patients, and caring for each other in the team were synonymous with emotional labour. For example, the activities of presenting a smiling face, listening and talking to patients, showing empathy and understanding and attending to the 'little things' that provided extra comfort and security was considered to be paid emotional labour (Smith, 1992). These 'little things' Smith (1992) contended, which were often taken-for-granted and not formally reported, were as important as intravenous infusions and managing pain in postoperative patients. These unreported components of emotion work ${ }^{26}$, Bone (2002) suggested only became evident and visible within the organisation when it was not carried out by the nurse and represented implicit unstated work which nurses could be judged for not doing.

Despite the need for emotion training, Smith (1991) noted that during the selection process nurses were chosen for their percieved suitability to be emotional labourers. This was based on the ideas set out in the "job prospectus which promoted the image of a caring young woman who wanted to be of service to others" (p. 77). Thus, nurse applicants, like the flight attendants in Hochschild (1983) work, were "introduced to the rules of the game of emotional labour" (Smith, 1991, p. 77) by the manner in which recruitment campaigns protrayed the nursing profession. Again, the

\footnotetext{
${ }^{26}$ Bone (2002) referred to the work that nurses undertook as emotion work not emotional labour.
} 
similarities in the demographics of successful candidates to become flight attendants and nurses were identified to be primarily white middle-class women thus, reinforcing the gendered nature of emotional labour (Hochschild, 1983; Smith, 1991, 1992).

Another aspect of emotional labour pertinent to nursing was the processes of 'connection' and 'involvement' which were considered fundamental to creating a trusting and intimate therapeutic nurse-patient relationship (Smith \& Gray, 2001a, 2001b). In contrast to the flight attendants work, Smith identified that nurses often had prolonged contact and emotional involvement with their patients where they were required to attend to the psychological and social aspects of caring by developing connections with patients. Nurses were expected to put extra effort, in addition to their normal nursing, into developing friendships, intimacy and trust with patients and "making patients feel at home" (Smith \& Gray, 2001a, p. 44). To do this, nurses were required to show their patients "a little bit of love" (Smith \& Gray, 2001a, p. 44) and be able to "relieve pain and suffering not by medical means but by compassion" (Smith, 1991, p. 77). This mandatory demonstration of compassion and friendship relied heavily on surface and deep acting techniques. However, Smith did acknowledge that due to the nature of the prolonged contact and emotional involvement with patients, nurses did engage in other strategies to manage their emotions and those of their patients, although this was not elaborated on in her work (Smith, 1991).

Over-involvement, as a result of giving 'a little extra' in conjunction with the nature of the continuous contact was a specific concern raised by Smith (1992). She observed that the continuous contact with patients increased the nurses workloads and intensified the emotional burden experienced. This left staff feeling undervalued. Furthermore, the stress involved in these close relationships that were formed, occassionally intruded into the private life of nurses, who were unable to emotionally distance themselves from their patients once at home. From these observations, Smith (1992) concluded that the cost of emotional labour was high for nurses and brought little value to nurses as emotional labourers. Moreover, she claimed that the intentions of emotional labour was made invisible by the economic and political priorities of healthcare that priviledged the most visible parts of nursing work which were 
considered to be physical care using technical skills. This disregard for the emotion work required, to establish 'connection' through 'involvement', Smith (1992) claimed added to the nurses' sense of being devalued.

The examination of emotional labour in the context of death and dying in ICU has provided new insights not only into the sources of stress experienced by staff but additionally the ways in which 'feeling rules' informed practice (Sorensen \& Iedema, 2009). Attitudes to death and dying, combined with the capacity to engage with the human needs of patients, influenced how emotional labour was experienced by ICU staff. In particular, emotional labour was considered to be a source of stress for staff who had not developed a positive attitude to death and were uncomfortable in their interactions with dying patients. The effect of caring for dying patients, not only affected the well being of staff themselves, but also the quality of care that patients received (Sorensen \& Iedema, 2009).

Furthermore, Sorensen and Iedema (2009) contended that feeling rules were not clear cut and the expression of emotions to dying patients and their families was a contentious issue within the ICU team. This was because not everyone agreed with the prescribed feelings rules expected in managing and responding to death and dying patients. The expression of emotions ranged from, denial of feelings and suppression of emotions, to open displays of feelings. Staff who believed that crying was an appropriate response to death feared the judgements of others and suppressed these emotions. In these situations there was a sense of injustice imposed on their feelings as staff were expected to unreasonably constrain the strong emotions that death evoked. Sorensen and Iedema (2009) concluded that all emotional labour was stressful but that the fear and stress that occurred in caring for dying patients was indicative of an individuals' unresolved fears of dying.

Emotional labour, as a reciprocal relationship, was identified in Li's (2002, 2004) work on symbiotic 'niceness'. Li (2002) demonstrated that dying patients were actively engaged in emotional labour of doing 'niceness' work, whereby the "niceness of nurses simultaneously require[d], fed on and $\operatorname{gr}[\mathrm{e}] \mathrm{w}$ from the niceness... of patients" (Li, 2004, p. 2577). By this, Li (2004) suggested that the effort patients themselves 
put into being nice to their nurses would be subsequently reciprocated and with greater intensity during psychosocial aspects of care. Similarly, Theodosius (2008) contended that emotional labour in nursing was an exchange based on interactions, trust and relationships formed between the nurse and the patient. This was because both the nurse and the patient were vulnerable to the actions of the other. The hospitalised patient was vulnerable to the amount and type of attention the nurse choose to give, whilst the nurse working in the consumer-orientated NHS was vulnerable to patient complaints (Theodosius, 2008).

This notion, that emotional labour was a reciprocal relationship, is at odds with Hochschild's (1983) work. Hochschild (1983) did not consider a reciprocating relationship as necessary in meeting the commercial aim of ensuring a positive experience for passengers. Thus, emotional labour was represented as being a nonreciprocal relationship where the passengers did all the 'taking' as a result of the power awarded to them by their ticket. This perspective overlooked the possibility that passengers and customers had a common interest in emotional labour. Customers who engaged in emotional labour and presented themselves as being nice people who were socially skilled in interacting with others had the potential to benefit from their efforts, such as being offered an upgraded seat or meal, or more regular refreshments and snacks during flight.

\subsection{Emotional labour: Interpersonal management of emotions}

In additional to the contribution emotional labour has made to understanding the gendered and caring role inherent to professional nursing practice, it has frequently been used to explain the contextual interpersonal level of managing the emotions of staff and their patients with specific diseases and illnesses (Frogatt, 1998; James, 1989; Li 2004, 2005; Smith, 1991, 1992; Smith \& Gray, 2001a), personal social qualities (Kelly \& May, 1982; Li, 2004, 2005; Staden, 1998) and sociodemographics (Kelly \& May, 1982). When applied to a variety of healthcare settings, how nurses engage in emotional labour and utilise its varied techniques, appear to be dependent on many factors, such as the patient population, the clinical setting, the culture and specific organisational rules of the workplace, and the attitudes of nurses. Most noticeably, emotional labour has been used as a protective strategy to maintain 
the nurses own emotional wellbeing when engaged in distressing care situations (Froggatt, 1998; Smith, 1992) or used to hide negative emotions towards 'undeserving' and 'bad' patients (Li, 2004, 2005; Staden, 1998).

Protective strategies have been mostly observed in the emotional labour and emotion work of caring for terminally ill patients in palliative care and hospice settings. In these situations nurses manage their emotions in a way to hide their feelings of distress, despair and failure as they become overly involved or too close to the dying patient (Smith \& Gray, 2001a). Various acting techniques and strategies have been identified as ways nurses manage their own emotions and those of their patients and families. In particular, these strategies have focussed on managing and maintaining a professional trusting and intimate relationship whilst preventing the consequences of the emotion work being done.

The examination of metaphors used in nurses' conversations by Froggatt (1998) has provided much insight into how nurses manage the emotions of themselves and dying patients in hospice settings. Froggatt (1995) demonstrated how emotional labour was part of nursing work through the metaphoric language of nurses' accounts of care. In particular, she observed that nurses perceived their bodies to be a 'container' for emotions whereby the mind (or upper container) acted as a lid to prevent the emotional content of the body (lower container) from leaving. Thus, nurses spoke in ways that described keeping a lid on or containing ones emotions: 'hide my feelings', 'hold it' and 'push it down' and also allowing feelings inside the body to be acknowledged: 'stirred up' or 'brings up feelings'. Froggatt (1995) further identified that stored in emotions needed to be released through the use of metaphoric phrases such as 'get it off chests' and 'got to come out.' This implied that the body as a container for emotions must not become too full or consequences such as feeling of being overburdened or emotionally drained may occur.

In Froggatt's work, nurses adopted distancing strategies to deal with the emotional work encountered when caring for dying patients to try and prevent becoming overburdened or emotionally drained. These strategies were 'switching on and off', 'hardening up' and 'standing back' which allowed individuals to 
"metaphorically and mentally distance themselves from the emotional threats engendered by their work" (Froggatt, 1998, p. 335). 'Switiching on and off' was a strategy nurses used to remove direct access to the emotions contained within their bodies. Nurses would turn on certain emotions when at work and shut them off again when going home thus, separating out their private and public life. This strategy implied that the mind was in control of the emotions and that nurses had the choice about whether or not to respond to their feelings.

'Hardening up' required nurses to remain present in the emotions of others, whilst a self-imposed impermeable barrier prevented any emotional effect on the nurse. Metaphors, such as, 'you have to keep a bit of a barrier up' and 'you have to draw the line', suggested that nurses created a barrier that prevented emotions from entering or leaving their body. This strategy required nurses to engage in emotion work while at the same time distancing themselves by "becoming impervious to the emotional angst around them" (Froggatt, 1998, p. 336). Again, it was implied that nurses had some choice as to whether they would deliberately not engage in the emotions arising from those around them.

The final strategy of 'standing back' required nurses to distance themselves mentally from the emotion work undertaken so that there was no effect on the nurse. This was achieved by metaphorically stepping back from a relationship or situation and at times positioning themselves physically in a different place to create distance. However, Froggatt (1998) noted that the frequent references to words and phrases such as, 'try' and 'most of the time', indicated that distancing strategies were not always possible to maintain. These distancing strategies served to protect nurses from becoming overly involved by creating a mental and physical space, thus reducing the risk of losing control of their emotions. In these situations nurses suppressed/ buried their emotions within the lower container of their bodies so true emotions were hidden from their patients and their families.

The use of distancing strategies raises two important points for consideration. Firstly, there is an assumption that the nurses cared about the patient and that they had formed a connection with them and their families. Thus, these strategies serve to 
protect them emotionally from emotional harm, stress and burnout. Secondly, the use of surface and deep acting techniques to achieve these distancing strategies were not elaborated on to understand what the consequences were to the quality and intimacy of the nurse-patient relationship.

More in fitting with Hochschild's (1983) conceptualisation, emotional labour has been used to demonstrate how nurses hide their negative emotions towards 'bad', 'undeserving', 'unpopular' and 'undesirable' patients, who nurses find unpleasant and take a dislike too (Kelly \& May, 1982; Li, 2002, 2004; Smith, 1991). A review of the literature by Kelly and May (1982) proposed that the reasons for disliking patients were varied in nature and included: negative attitudes towards certain clinical conditions such as incontinence; mental disturbance; mutilation; percieved deviant or rule breaking behaviour such as being drunk or not adhering to clinical regimes; percieved inappropriateness of patient responses to the care provision such as being unco-operative, unappreciative, demanding, attention seeking, immature, or rude ( $\mathrm{Li}$, 2002; Smith, 1991); and overacting emotionally to their current situation (Kelly \& May, 1982).

Appearing caring towards patients who nurses disliked as a component of emotional labour was examined in the work of Staden (1998) who explored the lived experiences of female nurses as emotional labourers. She identified that appearing caring was at the core of nursing work, where nurses were expected to suppress their feelings upon putting on their uniforms, as this symbolised the notion that they were "dress[ed] to care" (p.153). Despite this public display of appearing to care, nurses felt that it was not necessary to maintain this façade in the presence of their colleagues. Caring for 'bad' patients, Staden (1998) proposed, required a higher degree of emotional labour as nurses had to engage in deep-acting techniques, similar to those described in Hochschild's (1983) work where they were expected to change feelings within themselves. One technique used by a participant involved reflecting themselves onto the person they were caring for. By imagining the person in front of them was themselves, the nurse attended to the patient's needs in a way that was more fitting with the professional expectations of care. 
The 'undeserving' patient and the withholding of emotional labour was explored in Li's (2002) work on symbiotic niceness. She examined how the concept of niceness, as a component of emotional labour, was produced in the narratives of palliative care nurses. She identified that nurses talked about patients as being deserving or undeserving of the niceness that nurses incorporated into their practice. The undeserving patients were considered to be "unpleasant and unlikeable people" who were "neither charming, worthy, nor deserving" of niceness (Li, 2004, p. 2576). This was despite the nurses recognising that these dying patients had "troubled minds and bodies" (Li, 2004, p. 2576). During care nurses engaged in a type of niceness Li (2004) termed 'undeserved niceness', where nurses percieved that because of the patient's unpleasantness towards them they were only deserving of "obligatory care but minus nurses' niceness" ( $\mathrm{Li}, 2004$, p. 2577) Thus, in these instances nurses performed a perfunctionary role of care withholding the emotional labour of niceness.

The desire to withhold emotional labour was observed in Smith's (1991) work when nurses felt patients were not nice or were being rude to them: "I'll never say I particularly like all patients. You're told you have to be nice to them but I don't think you have to be if they're not being nice to you" (Smith, 1991, p. 77). This feeling that nurses had towards some of their patients led Smith (1991) to suggest that emotional labour was in fact withheld in certain situations.

In both Li's (2002) and Smith's (1991) work, nurses identified that their attitudes and behaviour towards patients were unprofessional when they failed to suppress their negative emotions and allowed them to surface inappropriately. Types of inappropriate behaviour that were reported included being impatient, stroppy, and abrupt during patient interactions ( $\mathrm{Li}, 2002$; Smith, 1991). In these situations, instead of responding to the patient professionally, nurses chose to avoid the patient, or not invest time in developing intimate relationships or offering compassion, and instead only provided essential perfunctionary care.

\subsection{Chapter summary}

Goffman's work acknowledged the complexity of social life across different cultures and settings. From the nursing and health literature it is evident that 
Goffman's work has been used substantively to construct new understandings of the social aspects of patient care and how health professionals manage and negotiate their private and professional self. The use of behavioural regions has provided insights into how care activities and behavioural expressions are organised and segregated both within the care environment and between professional and domestic domains. Similarly, the use of face and face-work has provided new ways of understanding how healthcare professionals present themselves to others during patient interactions and care situations and through the examination of emotions assists to make visible the emotional labour aspects of care. Despite this, much of this health literature tends to draw on only parts of Goffman's $(1959,1967)$ work and focuses on specific areas of practice or social encounters which has led to fragmented understandings of social interaction within different contexts of healthcare practice.

Hochschild's (1979, 1983) work has significantly contributed to the understanding of emotion, emotion work and emotional labour within the workplace. Hochschild's (1983) work primarily focused on exploring the relationship between emotions that are really felt and those that are acted out for the benefit of other people within particular social situations. From the nursing and health literature it is evident that Hoschchild's $(1979,1983)$ work has been used extensively to construct new understandings of the psychosocial aspects of care and the interpersonal management of emotions. The use of emotional labour has provided new insights into how healthcare professionals managed their emotions to maintain their own emotional wellbeing or to hide their negative emotions during care situations. Equally important has been its ability to make visible the undervalued tacit work of caring. Despite this, emotional labour in nursing is fundamentally different to that of Hochchild's (1983) flight attendants in several ways: the waged and unwaged dichotomy of private and public management of emotions can not be easily delineated; emotional labour is a reciprocal activity involving an emotional exchange that can be mutually rewarding; and emotional labour is based on trusting relationships.

Introduced in this chapter were the theoretical influences of Goffman (1959, 1967, 1969) and Hochschild (1983) where I described how their theories of social interaction and management of emotions have informed the understanding of 
interpersonal interactions during caring work. I have drawn attention to the specific ways in which emotional labour, behavioural regions, and face-work have been used to provided new insights into how healthcare professionals manage their own emotions during interactions involving care. In the next chapter, I provide a discussion of the methodological approach of focused ethnography, and methods of data collection and analysis that allowed for the understanding of how the attitudes and beliefs of healthcare staff specifically affected the care and social interactions between staff and fat patients. 


\section{CHAPTER FOUR: METHODOLOGY AND METHODS}

\subsection{Introduction}

The suitability of the methodological approach to any study is dependent on the research question. Previous studies outlined in chapter two have addressed the question of whether healthcare professionals were prejudiced towards fat patients. However, what is less understood is whether these self-reported weight bias attitudes influenced the actual behaviour of staff during bedside patient care. What existing literature has not explained was whether or how the attitudes of healthcare professionals specifically affected the care and interactions between staff and fat patients who were admitted to hospital with critical illness. Achieving this insight requires an in-depth description of the relationships and interactions between the healthcare professional and patient during care, and the thoughts, judgements and decisions made by staff during care situations. This type of understanding required a qualitative research approach that allowed for observation of behaviours and interactions.

As ethnography is both a methodology and a method I have chosen to present the philosophical underpinnings of the methodological approach taken and details of the methods of data collection and analysis within the same chapter. The detailed description of the process of conducting the study will be addressed separately towards the latter part of the chapter. In this chapter, I identify the study aims and objectives and provide a critical discussion of the methodological approaches considered during the research design and provide a rationale for selecting a qualitative focused ethnographic approach. Throughout this chapter, I will make apparent how my position as both a nurse and researcher within my own workplace influenced the study's methodological positioning and the research process of fieldwork. To do this I will draw on the works of other nurse researchers who have utilised ethnographic approaches within nursing research. 


\subsection{Study aims and objectives}

The aim of this study was to describe and explore the culture and influences within the intensive care setting in which nurses and doctors care for fat patients. The objectives were to identify the beliefs and perspectives of healthcare professionals that influenced the care of fat patients and describe how nurses and doctors care for fat patients in the intensive care setting.

\subsection{Methodological considerations}

Many forms of knowledge are situated and are constructed from certain people, for certain purposes, within a particular period of time. The situatedness of this knowledge reflects the social influences, such as stereotypes and prejudices, of that historical time (Gobo, 2008). This study recognises that ICU staff have different views of the world and of the people within it and that views and perspectives held can change over time. For this reason, this study has broadly adopted a social constructivist paradigm within the qualitative research tradition (Lincoln, Lynham, \& Guba, 2011). This philosophical position acknowledges that social reality is constructed by and between individuals, who generate their knowledge and meaning from their experiences and ideas (Lincoln et al., 2011; Koro-Ljungberg \& Greckhamer, 2005). This approach firmly supports the research question of understanding how ICU staff construct their realities of caring for fat patients from their own experiences and world views.

The source of knowledge is an important methodological consideration as each qualitative research approach provides a different world view of the area of research interest. Already identified in the literature was the gap in knowledge between self-reported attitudes/behaviours and actual behaviours of ICU staff when caring for fat patients. This gap in the knowledge has been identified in other studies which demonstrated that what we say and what we say we do, is in fact different to what we actually do (Deutscher, 1973; Gilbert \& Mulkay, 1983; La Piere, 1934). La Piere's (1934) pioneering work, which focused on the relationship and congruence of people's attitudes and behaviours, demonstrated that there was no relation between the two, as often people were inconsistent, unconscious and irrational in their actions. 
Thus, the importance of observation as a way to acquire knowledge of behaviours and actions cannot be underestimated. Equally, accounts made by social actors during interviews should not be treated "as an appropriate substitute for the observation of actual behaviour" (Heritage, 1984, p. 236). Therefore, a methodology, such as phenomenology, that relies on conscious experiences as experienced by the subjective or first-person point of view (van Manen, 2014) would not appropriately address the knowledge gap between self-reported attitudes/behaviours and actual behaviours. Instead adopting an ethnographic methodological approach that is premised on the cognitive mode of 'observation' as its primary source of information would most aptly address the knowledge gap (Gobo, 2008).

An ethnographic methodology was chosen for this study because its principle purpose is to provide an in-depth "thick description" (Geertz, 1973, p. 10) of a group culture from the perspective of its members through systematic observation of a group's activities, language and customs (Hammersley \& Atkinson, 2007). Culture, which is the broadest ethnographic concept, focuses on cultural behaviour and/or cultural knowledge of a social group (Fetterman, 2010). The type of research question often determines the focus of the study in favour of a behavioural (materialist) or knowledge (idealational) approach to observing and understanding the culture (Fetterman, 2010). In fitting with the research question the ethnographic approach of this study focuses primarily on the interpretation of the behaviours, and more specifically the interactions between staff and fat patients, that make up the culture. Thus culture, in this study can be defined as "the sum of a social group's observable patterns of behaviour, customs, and way of life" (Fetterman, 2010, p. 16).

\subsection{Ethnographic traditions}

Ethnographic research methodology was founded in the field of anthropology where traditionally researchers entered remote places as 'professional strangers' to study the unknown culture of indigenous people (Tuhiwai Smith, 2001; Van Maanen, 1995). Traditional ethnographic research was premised on the assumption that prolonged close contact with a social group, for months to years, in the form of field work would enable the researcher to develop a deep understanding of how the group 
lived and thought (Tedlock, 2000; Wolcott, 1995). During fieldwork, cultural data was collected from multiple sources which allowed cultural behaviours to be observed, cultural artefacts to be viewed in the context of daily interactions, and language to be heard and understood (Hammersley \& Atkinson, 2007). Ethnography provided the opportunity to better understand the beliefs, motivations, and behaviours of cultural groups through the new perspectives that could be found (Tedlock, 2000).

The application of ethnography to other disciplines, such as sociology and healthcare, has questioned some of the more traditional principles and methods of ethnographic research (Knoblauch, 2005; Savage, 2006). In particular, many applied disciplines, such as nursing, focus their attention on understanding the meanings within their own society and exploring specific social problems (Knoblauch, 2005). This type of ethnographic enquiry is fundamentally different in two ways. Firstly, the culture being observed is not foreign and unfamiliar, and challenges the idea that through participant observation "one comes to understand something by seeing it as an outsider" (Sanday, 1979, p. 528). Secondly, instead of understanding the "totality of all learned social behaviour of any given group" (Thomas, 1993, p.12), specific identifiable activities, social problems, or an individual within the larger social group becomes the focus of the research (Fetterman, 2010). These applied ways of using traditional ethnography has led to new ethnographic styles of researching specific social problems and how it is articulated, researched, written about and used to inform practice (Fetterman, 2010). Critical, institutional and focused ethnography are examples of applied ethnographic methodologies that support particular ways of researching a particular social group. However, the knowledge acquired from these different approaches provide diverse insights into the culture observed and serve different sociological, political and economical purposes.

Critical ethnography is an approach that is overtly political and specifically attempts to expose social, political and material inequalities in an effort to elicit change (O'Reilly, 2009; Madison, 2005; Thomas, 1993). The intention of the research is the emancipation of cultural members from ideologies that are not to their benefit and not of their creation (Thomas, 1993). The ethnographer focuses attention on exposing hidden agendas, challenging oppressive assumptions, describing power 
relations and critiquing taken-for-granted behaviours of the cultural group. In this way, critical ethnography facilitates the ability to disrupt the status quo, and unsettle both neutrality and taken-for-granted assumptions by examining the power relations and influences that affect the experiences of the group (Street, 1992).

Institutional ethnography is an approach concerned with the political and economic contexts of organisations and specifically explores the social relations that structure people's everyday lives (Smith, 2005). It is used to examine the macro and micro connections amongst local settings of everyday life, organisations and translocal processes of adminstration. The intention is to reveal how social systems and institutional relations shape individual experiences and culture (Gobo, 2008). The ethnographer focuses attention on ordinary daily activities of the organisation by mapping the translocal relations that co-ordinate people's activities within the institution. By doing so, the macro-level political and organisational knowledge is revealed to understand how this influences the micro practices observed in daily life (Smith, 2005).

Focused ethnography is an approach that explores a distinct issue or shared experience within a culture and in specific settings, rather than throughout entire communities (Cruz \& Higginbottom, 2013; Fetterman, 2010; Higginbotton, Pillay, \& Boadu, 2013). It examines specific cultural perspectives held by a subgroup of people within a context-specific and problem-focused framework (Higginbottom et al., 2013). The intention is to enhance and understand specific knowledge of practices that occur within distinct professional cultures or subcultures. The ethnographer focuses attention on the specific activities and shared features of individuals in the subgroup when engaged in practices related to the distinct issue (Cruz \& Higginbottom, 2013). In this way, focused ethnography facilitates the ability to acquire specific knowledge about an identified problem which can inform future practices.

Focused ethnography is a widely accepted and used methodological approach to understanding the social phenomenon of nursing and healthcare practice (Cruz \& Higginbottom, 2013; Higginbottom, 2011; Higginbottom et al., 2013; Scott \& Pollock, 2008; Smallwood, 2009). It has been used in nursing to explore: specialised teams 
within healthcare services (Smallwood, 2009); different aspects of nursing practice (Scott \& Pollock, 2008); the provision of care to particular patient populations within specialised services (Tzeng, Yang, \& Tzeng, 2010); nurse-patient relationships during certain modes of therapy (Spier \& Wood, 2010); and nursing workforce experiences (Higginbottom, 2011). In each study the purpose was to explore key components of a subculture that informed larger nursing phenomena.

Much consideration must be given to the ethnographic approach adopted within a study as the knowledge acquired from these different approaches provide different ways of understanding the culture observed and serve different sociological and health purposes. For this reason, the most appropriate approach must always be guided by the intentions of the research. Given the nature of the research question, the obvious choice for this study was to adopt a targeted approach using focused ethnography. Key characteristics of focused ethnography that support the methodological approach taken include: the type of research question; the nature of the participant observation; having established background knowledge; and specific knowledge held by participants (Cruz \& Higginbotton, 2013; Knoblauch, 2005).

The study research question aimed to explore the distinct issue of how fat patients were cared for by staff within the specific setting of the intensive care unit. Thus, the purpose was not to explore how all patients with critical illness were cared for by intensive care staff, instead a subculture within the wider social group was of interest. The nature of participant observation differed from traditional ethnography in that it was characterised by relatively short term intermittent field visits (Knoblauch, 2005). As participant observation would be dependent on the admission of fat patients the method of data collection would be one of episodic periods of intense participant observation (up to 16 hours a day) where large amounts of rich data would be accumulated. Knowing where to focus the attention of these observations to explore the specific issues required background knowledge of the research field (Higginbottom et al., 2013). Based on my nursing experience of fat patients, intensive care practices, and the research site, I was very familiar with many aspects of the culture and it was precisely this knowledge that led me to develop the research question. Equally, this background knowledge meant that I was aware that intensive 
care staff held specific knowledge of how they cared for fat patients which was not evident in the existing literature. For these reasons, a more targeted approach using focused ethnography was more applicable than more traditional forms of ethnography. This chosen approach enabled the specific issues and challenges of fatness that affect different aspects of ICU practice to be explored using intensive intermittent methods of data collection to elicit specific participant knowledge (Cruz \& Higginbotton, 2013).

\subsection{Theoretical influences}

There is a continuing debate regarding the role that theory has to play in ethnographic research, with particular tension surrounding the perspective of whether ethnographic approaches should be theory driven or theory generating (Anderson, 2002; Wacquant, 2002; Wilson \& Chaddha, 2009). Theory driven, deductive, ethnographies focus their fieldwork in a 'context of validation' where data is used to test, advance or explain empirical assumptions derived from theoretical positions (Wilson \& Chaddha, 2009). Critical ethnography would be an example of this approach, where critical social theory is applied to the data in a way that examines the power structures inherent in the social context under study. A criticism of this approach is that the rigid commitment to a theory forces the subordination of the cultural complexities in the field to that theory where data is forced to either fit or be dismissed (Anderson, 2002; Duneier, 2002). Within critical ethnography, the overemphasis on the marginalised group can potentially lead to dismissing or ignoring important data of how other groups who are not considered to be marginalised view these groups (Thupayagale-Tshweneagae, 2008).

Alternatively, a theory generating, inductive approach, as used in this study design, requires the ethnographer to be familiar with the influential theories that support the field of research. These theoretical concepts are not taken and applied in their entirety to the research process or during data analysis but are used by ethnographers to enhance their explanatory and interpretative powers (Anderson, 2002). Thus, inductive ethnographers focus their fieldwork in a 'context of discovery', where an attempt is made to uncover the relationships that have not been explicitly supported or explained by theory (Wilson \& Chaddha, 2009). The ethnographer 
therefore, enters the field "with a certain sociological sophistication, even a theoretical perspective that, as the fieldwork proceeds, helps to formulate questions concerning the social organisation of the subjects and their settings" (Anderson, 2002, p. 1536). Hence, theoretical insights are used to inform the interpretation of the data generated in the 'context of discovery' by integrating the findings of the study with theoretical arguments from the researcher's own theoretical knowledge to make sense of the data (Wilson \& Chaddha, 2009). The prominent theories that have emerged during the analysis of this study were Goffman's $(1959,1967,1969)$ dramaturgical perspective of self in social interaction, and Hochschild's (1983) emotional labour (see Chapter Three).

\subsection{Reflexivity}

Reflexivity recognises that the researcher is inescapably part of the social world being researched (Gobo, 2008). It is a fundamental component of the ethnographic research process and requires the researcher to critically reflect on the self as an instrument (Lincoln \& Guba, 2000). Reflexive practices focus on critical self analysis of the nature of the relationship between the researcher and the participants (Gobo, 2008; O'Reilly, 2009), the ability to make explicit the role assumed during the study (O'Reilly, 2009), and the way in which the researcher's subjectivities influence the research process and written product (Allen, 2004; Bradbury-Jones, 2007). Equally, reflexivity involves asking questions of the data which would expose how these biases have affected the reporting of the social phenomena observed (Hertz, 1997; Reinharz, 1997).

Many aspects of the research process are scrutinised in this way, in particular: the concern with how the field of study is perceived through the assumptions, theory, and interpretive lens of the researcher; consideration of the individual's history and biography, and theoretical perspectives (Allen, 2004); consideration of the extent to which active participation in the field effects the phenomena being researched (Hammersley \& Atkinson, 2007); and an acknowledgement that the research field will have an effect on the researcher (Coffey, 1999). Such considerations and transparency of the research process are regarded as an integral component to the ethnographic rigour and validity of the study findings (Allen, 2004; Seale, 1999). The use of 
reflexivity, has had a pivotal role in articulating how my position as both a nurse and researcher, within my own workplace, influenced the philosophical and methodological positionings of the study and the research process of fieldwork. These considerations have been addressed throughout the subsequent parts of the chapter in order to provide transparency of process, findings and written representations.

\subsection{Participant observation}

Ethnographic methodology gives priority to observation as its primary source of information and comprises of two research strategies: non-participant observation and participant observation (Gobo, 2008). Non-participant researchers observe from a distance, do not participant in any activities, and avoid any interactions that may influence the behaviour of interest (Gobo, 2008; Gold, 1958; Sarantakos, 1998). In contrast, participant observers directly interact with the study participants and engage in all the group's activities, ceremonies and rituals in order to understand how people interpret various situations (Bowling, 1997; Gobo, 2008). Despite these clear methodological positions of generating data through observation, the reality of the practice setting and the researcher's own identity often means that they merge together (Turnock \& Gibson, 2001). This has led to much debate about what separates participatory and non-participatory roles, generating multiple definitions and categories of this continuum of observation (Gobo, 2008; O’Reilly, 2009; Sarantakos, 1998).

What has been agreed by ethnographic researchers is the importance of describing, through reflexive practices, the actual role that the researcher undertook within the field (Turnock \& Gibson, 2001), by detailing the selective participatory involvement in the group's normal activities (Pretzik, 1994). In careful consideration of the two identities that I took into the study site, and the context of the research question, I choose to adopt an 'observer as participant' role (Gold, 1958), whereby observation was favoured over participation (Hammersley \& Atkinson, 2007). This position of 'observer as participant', where observation dominates the participatory role, has been adopted in other nursing ethnographies (Arber, 2006; Asselin, 2003; Coombs, 2004; Page, 2006). 
My intention, as an 'observer as participant', was to primarily observe the clinical practices of ICU staff when caring for fat patients at the bedside. At the same time I intended to participate in all non-clinical activities as if I was still a staff member working at the study site. This would allow me to immerse and expose myself to as many different observational opportunities as possible. This chosen level of participation has been supported in the recent research conducted by Evans, Pereira, and Parker (2013) who examined how nurse participants reacted to the nurse researcher who did not 'help out'. This non-participatory position confused the nurse participants and made them feel anxious in their practice. Furthermore, nurses tried to resolve the issue by either attempting to include the researcher into the group or pushing the researcher away (Evans et al., 2013). This dissonance between the dual identity is often reduced by offering a positive contribution to the workplace, instead of being merely "an exploitive interloper” (Gerrish, 1997, p. 27).

While participation concerns the researcher's involvement in the field, observation concerns the research participants' perceptions and knowledge of the researcher and the research being conducted (Gobo, 2008; Turnock \& Gibson, 2001). How the observation is conducted broadly fits into three distinguishable types: covert; semi-covert/overt; and overt. Covert observations occur in situations where the participants are unaware of the researcher's identity, the purpose of the research, or in fact that they are the subjects of a research study (Bowling, 1997; Gobo, 2008). Semicovert/overt observations occur in situations where not all participants are aware of the research (Gobo, 2008). Overt observations occur in situations where the identity of the researcher and purpose of the study is known (Sarantakos, 1998), and participants have formally consented to participating in the study (Gobo, 2008). There are many advantages, disadvantages and ethical considerations to the type of observation used within any ethnographic study which have been widely debated in the literature (Gobo, 2008; Lathlean, 1996; O’Reilly, 2009; Patton, 1990).

Given my social positioning within the group, overt or open observation was the natural choice of observation, whereby the participants were fully aware that they were being observed (Couchman \& Dawson, 1995), the identity of myself as a researcher was known (De Laine, 1997), and the ICU staff, fat patients and external 
healthcare professionals, such as surgeons, physiotherapists and social workers, knew the purpose of the study (Sarantakos, 1998). Thus, my role as the observer and the purpose of the observations were made explicitly clear to the participants of the study.

\subsection{Insider research}

Through the role of participant observation the ethnographer attempts to understand the cultural group by becoming an "insider while consciously and objectively describing and analysing the events as an outsider" (Roper \& Shapira, 2000, p. 116). This concept of 'insider-outsider' status is fraught with methodological concern, both in terms of the process and written representations of the cultural group (Bonner \& Tolhurst, 2002; Labaree, 2002; O'Reilly, 2009). Furthermore, it is compounded by the disagreement amongst ethnographers of what this actually means (Labaree, 2002).

Traditionally, researchers were foreign and 'outsiders' to the culture under study and through prolonged engagement would become socialised into the cultural group and gain insider knowledge and understanding (De Laine, 1997). However, a recent increase in the number of studies in which the researcher is already an 'insider' and becomes the instrument of the research process has focused much interest into the comparative merits of 'insider' and 'outsider' research (Allen, 2004; Bonner \& Tolhurst, 2002; O'Reilly, 2009). This has generated a debate about whether familiarity or strangeness allows for a more objective reality to be observed (Burgess, 1984). This argument is based on the assumption of an existence of an objective reality which can be scientifically observed (Allen, 2004). Researchers who favour the 'outsider' position base their arguments on the idea that the research, because of the absence of any affiliation with the cultural group, will be bias-free (Allen, 2004), the researcher as an impartial observer is more likely to question what others see as ordinary (O'Reilly, 2009), and seeks further clarity instead of assuming and interpreting the observation using their own knowledge (Gerrish, 1997). The major criticism of outsider research is the time and effort necessary to form relationships and gain the trust of the participations, and to achieve enough understanding of the culture and jargon of the setting to proceed with meaningful observations (Gerrish, 1997). 
Opposing this view, advocates for 'insider' research argue that researchers who are immersed in their own field of study can authenticate their observations and accounts (Allen, 2004; Griffith, 1998), have a privileged fundamental understanding about what is happening (Asselin, 2003), and a prior knowledge of 'rich points' of observational interest (Bonner \& Tolhurst, 2002). This familiarity allows the insider to have linguistic competence in which to ask more subtly posed questions on more complex issues (O'Reilly, 2009), be able to assess the impact of their presence on the culture being observed and subsequent changes to normal practice (Bonner \& Tolhurst, 2002), and be able to "get beyond the ideal to the real, daily, lived, and back-stage experiences" (O'Reilly, 2009, p. 114). The major criticism of insider researcher is that the insider knows too much and simultaneously not enough about their culture, as an insider, to objectify its meaning (Eipper, 1998). In other words, insiders are too close, too involved and lack detachment with the setting for the "unfamiliar and exotic to arouse curiosity" (O'Reilly, 2009, p. 112).

The dichotomy and complexities of the insider and outsider positions held by nurse researchers undertaking healthcare ethnographies have been explored in the nursing literature (Arber, 2006; Bonner \& Tolhurst, 2002; Coombs, 2004; Hoare, Buetow, Mills, \& Francis, 2013). Despite the many inconsistencies in how nurse researchers position their studies, there does appear to be a tendency to broadly adopt one of three positions depending on the familiarity with the setting and specialty, and the identified role and nature of the research. These positions are the outsider, insider/outsider, or insider. Nurses tend to adopt an outsider position when the area of research interest is unfamiliar to their normal area of work or specialty practice (Bonner \& Tolhurst, 2002). In this instance, other than being a nurse, they have no prior familiarity or knowledge of the practices of that specialty or of the research participants. Therefore, they consider themselves more of an outsider in the research process.

Nurses who adopt a dual insider/outsider perspective often research a nursing area of familiarity but consider their researcher status as equally influential on the research process (Coombs, 2004). In this situation, nurse researchers consider 
themselves to have insider knowledge of general practices of their specialty which they use to engage with the nursing team, whilst concurrently focusing on their researcher role by seeing the social world as an outsider. Therefore, because they have insider knowledge but are often unknown to the nurse participants prior to the study, the boundaries of insiderness become situational and defined by the perceptions of the research participants (Labaree, 2002). Hence, insider/outsider researchers often "live simultaneously in two worlds" (Coombs, 2004, p. 46), and consider themselves equally an insider but also an outsider as they have entered the setting as a researcher.

The insider position is usually adopted by nurse researchers who are not only researching their own specialty practice areas of nursing but also their own workplace and colleagues (Asselin, 2003; Cudmore \& Sondermeyer, 2007; Griffiths, 2008; Simmons, 2007). In these instances, the nurse researchers are extremely familiar with the setting and specialty knowledge of the daily routines of the place, and of the research participants prior to engaging in the study. This intimate knowledge and preexisting close relationships are used strategically to inform their fieldwork. Although, they have taken on a new role of researcher they consider themselves more of an insider in the research process due to the close relationships and expectations of their colleagues that exist during the fieldwork process.

Despite these difference perspectives, what has been agreed is the importance of using reflexivity to understand the tensions that existed within the study. This is both in terms of familiarity and distance, and the dual nurse-researcher identity (Allen, 2004; Cudmore \& Sondermeyer, 2007). In doing so, the researcher's positionality of insiderness and outsiderness, as a process of the research, is articulated rather than simply assigning a title to the researcher (Labaree, 2002). This is achieved by making explicit the roles undertaken in the field and how this may have impacted on the research process and findings (Allen, 2004; Labaree, 2002).

This study has adopted an insider perspective as the research site was my current place of work, and had been for the previous seven years. During this time I had developed significant amounts of familiarity with the clinical setting, and the activities, routines and rituals that formed daily life working as a senior intensive care 
nurse. Although, I have positioned myself and this study firmly within an insider position I was still consciously aware of my outsider role as a researcher within this setting and how that came with new ways of observing and scrutinising practice. Secondly, I was cognisant that being an insider to my culture did not necessarily mean that I had intimate knowledge of the participants 'situated' experiences (Kanuha, 2000). In fact it was this 'situated' experience of my colleagues that was of interest to me in understanding how fat patients were cared for in ICU.

The insider, once situated in the field, attempts to treat the familiar world of its 'members' as anthropologically strange, to expose its social and cultural construction (Walsh, 1998, p.218). This can be particularly difficult when a researcher is studying the group in which they hold membership and owe allegiance, but nevertheless represents an ideal that must be strived for (Walsh, 1998; Wolcott, 1995). This raises a pertinent methodological question about whether an insider can do effective research in an area in which they hold membership and owe allegiance. Wolcott (1995) suggests that the answer to this question lies in recognising what an insider's perspective can offer and whether only an insider can get an inside view. In response to this, Griffith (1998) suggests that an insider brings "authenticity to the research that is impossible to reach from the outside" (p.375).

In acknowledging the benefits of insider ethnography as outlined above, and the idea that an insider's perspective is culturally accustomed to that of its group, this study is positioned from the viewpoint of the insider researcher. Moreover, an insider who is studying their own cultural group to which they belong and participated in immediately prior to commencing the fieldwork. Thus, the tensions between both familiarity and distance, and dual nurse-researcher identity, have been accordingly considered and are addressed through reflexivity in the latter part of this chapter.

\subsection{Representation}

Writing is central to the process of ethnographic research but how the ethnographer reveals the participants and their culture, presents the relationship formed with the participants, and the way in which the researcher appears within the text requires substantial consideration (Reed-Danahay, 2002; Van Maanen, 2011). In 
doing so, the ethnographer positions the written account from an 'emic' or 'etic' perspective. The terms 'emic' and 'etic' refer to the modes of understanding (Barrett, 1991), and the alleged nature of the knowledge retrieved, as opposed to the knowledge source of how it was obtained (Willis \& Elmer, 2007). These modes of understanding significantly inform the perspectives to which the ethnographic accounts are written. In this way, the written accounts must align with methodological positions and perspectives adopted throughout the research process.

The emic mode, referred to as the actor-orientated (De Laine, 1997) or insider perspective, aims to provide descriptive accounts that represent the culture in ways that are meaningful to the participant's own culture, are culture specific (SeymourSmith, 1986), and typically embody what is common sense within the culture under observation (De Laine, 1997; Willis \& Elmer, 2007). In alignment with the philosophical and methodological underpinnings of this research, the study has adopted an 'emic', insider perspective to understanding and representing the culture within the intensive care setting.

During fieldwork, ethnographers listen to the different voices they encounter, which sometimes can present conflicting perspectives of the same observed phenomena or varied versions of the percieved realities (Clifford, 1983; Davis, 2000). Consequently, the ethnographer is confronted with the difficult task of dispersing the author's authority by providing a selection of diverse perspectives, subcultural values, and voices in the final text (Davis, 2000). In the creation of representation “ethnographic writings provide a glimpse into the author's privileged access to the character's thoughts, feelings and motives, as well as their overt speech and action" (Emerson, Fretz \& Shaw, 1995, p.223).

During the construction of the accounts the author must decide whose voices will be present and heard, thus determining the reality of that cultural group (Davis, 2000). Conventionally, ethnographers made a clear distinction in the written text between the author and the cultural members by representing the participants as the "object of the ethnographer's gaze" (De Laine, 1997, p.111). As such, the voices of the cultural members were muted by the single authorship of the privileged 
ethnographer (Wing-Chung, 2008) through artificially synthesising univocal texts (Clifford, 1983). However, the approach taken in this study identifies the story teller and questions the authority, knowledge, presence, voice and values of the author through reflexive practices (De Laine, 1997). This allows for a 'decentred' representation of the culture which limits the power of one voice and encourages the plurality of voices (Marcus \& Fischer, 1986). Different voices dominate the text within different places and contexts of the written accounts of this research. For example, the findings of this thesis are dominated by the multiple voices of the participants as their 'situated' experiences of caring for fat patients are presented. In contrast, my authorial presence dominates the methodological positioning of the thesis and reflexive discussions where I discuss the relationships and experiences which generated the data in the field.

\subsection{Study design}

The design of this study was focused ethnography and involved engaging in the experiences of nurses and doctors as they cared for critically ill fat patients in the intensive care setting. Ethnographic methodological principles and methods guided data collection, analysis and the written representation of social group researched. The following sections of this chapter describe in detail how this study was conducted thereby providing rigour and transparency to the process, findings and written representation of the research.

\subsection{Study setting}

The setting for this study was an 18 bedded tertiary level III $^{27}$ ICU in New Zealand. This unit was capable of providing comprehensive intensive care for indefinite periods of time where complex multi-system life support could be administered (College of Intensive Care Medicine of Australia and New Zealand, 2011). All patients admitted to the unit were under the care of the intensive care specialists regardless of their presenting illness, and on discharge from the unit were transferrred to the care of the specialty primary team. The ICU served a population of approximately one million people over a $300 \mathrm{~km}$ radius and operated an intensive care

${ }^{27}$ See Section 2.2 . 
flight retrieval service. At the time of the study there were 139 staff members, consisting of 110 registered nurses, 4 healthcare assistants, 20 doctors and 5 adminstration support staff.

\subsection{Ethical approval process}

Following conceptualisation of the research study as ethnography, I consulted with key stakeholder's within my local District Health Board (DHB) about undertaking the study within my own ICU. Initially, this involved individual meetings with the Director of Nursing, and the medical and senior nursing teams at this chosen site. These conversations focused on affirming the need for the study and then gaining support for undertaking it within my own workplace. It was at this early stage of developing the research and confirming the study site that the limitations and disadvantages of researching my own colleagues' practice were discussed with the staff on the unit.

Staff perceptions, expectations and level of trust in the researcher have been cited as important considerations when choosing to research colleagues in practice (Asselin, 2003). Staff perceptions and expectations of the researcher can affect how participants interact and the quality and quantity of information they are prepared to share (Asselin, 2003). In keeping with Asselin (2003), I felt that if staff in the study sites believed that observations and accounts of their practice would be evaluated, critiqued and reported back to senior doctors and nurses, staff may not be as willing to participate. Likewise, if staff perceived hidden agendas in the study, they might not be as supportive and open to sharing thoughts, feelings, and frustrations for concern of being reprimanded. Thus, the depth and breadth of data needed to achieve an accurate representation of the culture within the ICU when caring for fat patients might not be obtained. It was my belief that, resultant of my senior nursing profile, the integrity that I have always sought to demonstrate on the unit, plus my rapport held with staff, meant staff were fully supportive of my request to observe them in practice.

All key stakeholders were fully supportive of the project, despite voicing some apprehensions about what the study might reveal. Letters of support for the study 
from these stakeholders, in addition to the locality assessment ${ }^{28}$, were sent to the Health and Disability ethics committee. The Research Advisory Group for Māori (RAG-M), the Whānau Care Services ${ }^{29}$ and the Pacific Strategic Advisory Group (PSAG) were fully consulted in the development of the study and ethics approval processes. The RAG-M endorsement process involved meeting with the hospital Māori clinical leader to discuss the study and subsequently submitting an application to the RAG-M committee for acceptance. As part of this endorsement the Whānau Care Services were consulted and involved in the study as outlined in the endorsement letter (see Appendix 1). The PSAG consultation was not compulsory for ethical approval; however, in acknowledging that Pacific Island patients were likely to fit the inclusion criteria in disproportionately high numbers, I chose to follow similar consultation and endorsement processes as outlined above. I presented my proposed study at the PSAG regional committee meeting and subsequently received a letter of endorsement. All support letters and endorsements were submitted to the ethics committee as part of the application process. I received ethical approval in mid 2009 from the Central Regional Ethics Committee (see Appendix 2).

An amendment was made to the original ethical approval application once data collection commenced. This was because the initial consent process was for nursing staff and patients only; however, it soon became apparent through participant observation that doctors and other healthcare professionals were influential in shaping ICU culture. Therefore, I applied for ethical approval to be able to interview other healthcare professionals. This approval was granted in early 2010 (see Appendix 3).

The key principles of ethical conduct, such as beneficience, non-maleficence, justice, and respect were addressed within the ethical approval documentation. A condition included in the ethical approval application was that I would take unpaid leave from work during the data collection period. There were two reasons for including this condition in the application process. Firstly, and most importantly, I

${ }^{28}$ A locality assessment is a unique requirement of the New Zealand research ethics process whereby the study site completes an assessment of its ability to fulfil the research requirements of the proposed study (Health and Disability Ethics Committees, 2005).

${ }^{29}$ Whānau Care Services are a Māori led service which provides support to Māori patients and their family/whānau during hospital admissions. 
would be relieved of any employment responsibilities as a senior nurse to report to management or personally deal with any nursing concerns that occurred during the study period that would normally fall under my employee role description. Additionally, this would further allow me to identify myself as a researcher. Furthermore, at the time of preparing the ethical application other District Health Board's in the region were unwilling to complete locality assessments and consent to research being conducted by their paid nursing staff where direct observation of practice would occur; possibly for the reasons discussed above. Therefore, I preempted and addressed this issue at the time of the application to the ethics committee.

Although my employment responsibilities were waived for the duration of the study, I still had to abide by my professional responsibilities to maintain public safety in accordance with the Nursing Council of New Zealand (2012) codes of conduct. This dual identity of the nurse researcher creates tensions and conflict between the roles and ethical responsibilities of the investigator (Casey, 2004; Houghton, Casey, Shaw, \& Murphy, 2010). On the one hand, nurses have a duty of care to patients, while on the other, as a researcher they are committed to ensuring the integrity of the scientific methods of the research process (Holloway \& Wheeler, 1995; Houghton et al., 2010). This conflict of dual identity draws attention to the divided loyalties of the nurse researcher, whereby decisions have to be made as to whether greater allegiance is given to the participants or the research (Jones \& Jack, 1999).

In practice, researchers in this dual role have identified that they would only intervene in care if the patient or client was in danger (Davies, Ellis, \& Laker, 2000) or it was an emergency situation (Elliott \& Wright, 1999), supporting the idea that nurse researchers should always revoke their researcher role when the patient's safety is threatened (Holloway \& Wheeler, 1995). However, what is often not made explicit is what constitutes danger or emergency situations, highlighting the importance of developing research-specific ethical protocols to guide when the researcher will intervene during the research process (Cerinus, 2001). Most commonly, ethical protocols that guide researcher intervention address situations where a suitably qualified staff member is not present and a patient is experiencing a life-threatening 
event, or their safety is at risk from a fall, maltreatment or inappropriate treatment by a staff member (Houghton et al., 2010).

In considering my dual identity decisions had to be made prior to the beginning of fieldwork about when, to what extent, and for what purpose I would intervene during observations of care (Casey, 2004; Houghton et al., 2010). All stakeholders were in agreement that I would only intervene, as a nurse, if I observed a patient in 'imminent danger' of being harmed. Clarity around this concept of 'imminent danger' was important as I had to feel there was a way to observe my colleagues as a researcher yet maintain my professional responsibilities. I described 'imminent danger' to stakeholders and staff participants via the following examples: if I observed potassium chloride (a commonly administered but lethal drug used in intensive care) being administered in a way which would induce a cardiac arrest then I would intervene. The patient in this situation is clearly in danger. This example was often met with amusement, as ICU nurses are all too aware of the dire consequences of this drug error. As a counter-example I informed nurses that if I observed a staff member swearing in front of a patient then I would not intervene. My argument here is that although the nurse was acting unprofessionally and potentially causing offense to the patient, the patient in this event was not in any danger. Therefore, throughout the fieldwork my decision to intervene would be guided by situations in which the patient's safety was imminently at risk.

\subsection{Participant selection, recruitment and sampling strategy}

Participants included in this study were fat patients and the ICU staff who cared for them. However, the primary participants were ICU doctors and nurses. There were two important factors in the decision to focus my attention on the ICU staff. I was cognisant of the research question that focused on the 'situated' experiences of ICU staff in caring for fat patients. Due to the anticipated nature of the patient's condition (sedated and ventilated) on and during their admission it was percieved unlikely that they would be able to actively participate in the research process. For this reason I choose not to recruit patients for interviews. However, many of the patients were, in fact, conscious for the majority of their admission and their conversations in the field became part of informal interviews during the fieldwork. In 
this respect fat patients were instrumental in locating the fieldwork observations to be made and were therefore part of the field.

All staff within the ICU were involved in the study unless they chose not to consent to participate. Staff and patients were consented into the study through different processes. Staff were consented prior to the data collection period whilst patients were recruited and consented to be observed at the time of data collection. My aim was to consent at least $80 \%$ (approximately 100) of the staff ahead of the observation period so that when a patient consented I could begin immediate participant observation. Written consent for observation and interviewing was arranged on an individual basis to allow nurses to talk openly and confidentially about any concerns they might have with the study. These sessions took approximately 30 minutes each as I outlined key conditions of the ethics approval, what that meant for participants, and discussed the content of the information sheets before obtaining written consent (see Appendices 4 and 5).

An important part of the discussion was how I would gather data from observations and represent these in my findings. Given that I would be observing social interactions between staff and patients it was important to clarify where and what data would be collected. This was explained to the participants in the following way: If staff and patients were aware that I was present and observing them then conversations that formed part of the care/social interaction would be considered data. However, if participants did not know I was present, such as standing in a corridor out of view then this would be considered 'overhearing', as the conversation being heard was without the speakers knowledge or intention and would therefore not be used as data. This was explained in the consent documentation as 'at no point during the study will conversations that are overheard during periods of data observation be recorded and used as part of the data collection' (see Appendix 4).

Additionally, during the consent process I talked openly about the collaborative approach that I would take to establish the 'situated' experiences as viewed by the staff, especially if undesirable behaviours were observed. Additionally, I stressed that the behaviour of one did not make a culture and that I was focusing on 
the collective culture. Therefore, an individual's behaviour would not be sensationalised and misrepresented as the unit's culture for the purpose of exposing nursing practice and academic gain. Only one nurse initially refused to be in the study but once the participant observations began and she could see how the observations of practice were being conducted, the nurse approached me to discuss being in the study. Although, staff were happy to be observed in practice, some nurses did not want to be interviewed, primarily because they didn't want to hear their own voices on audiotape. None of the medical team refused to be a part of the study.

Patients who were admitted to the study site who had a BMI $\geq 40 \mathrm{~kg} / \mathrm{m}^{2}$, were not undergoing weight loss (bariatric) surgery and expected to remain in the unit for more than 12 hours were eligible for the study. Patients who were undergoing weight loss surgery were excluded at selection for two reasons. Firstly, their primary focus for being in hospital was surgical intervention for weight loss which was managed within a strict protocolised regime that was independent of ICU practices. Secondly, bariatric surgical patients are mobile, well, and have been physiologically optimised prior to their elective surgery. This was an important consideration in determining the patient population, as at the time of the study a contract to undertake bariatric surgery was being negotiated with the proposal to use ICU for extended high dependency post-operative monitoring for 12-24hours.

Identification and subsequent consent of patient participants occurred through two processes. Patients were either identified from their pre-admission cardiac surgery assessment forms and consented for the study the night before surgery and admission to the unit or, identified on admission to the unit and consented following confirmation of the inclusion criteria. There were eight patients identified as eligible for the study, of which seven consented. The other eligible patient was unconscious on admission, and during conversations with the family I became concerned about whether he would want to participate if he had the choice. This was further complicated by the severity of his head injury and the potential for him to never be in a position to formally consent. Therefore, I chose not to proceed any further with the enrolment process. 
At the time of consent patient participants were reassured that their care would not be affected if they chose not to participate in the study or if they chose to withdraw. During this consent process I discussed the information contained within the patient information sheet (see Appendix 6) and answered any queries patients had, before completing the written consent form (see Appendix 7). I had some trepidation about approaching the patient participants as the criteria for enrolment might have caused offence to people who were sensitive about their weight. However, patient participants were extremely open in discussing their weight at the time of consent.

Although not necessary, I gained medical consent for each patient to be enrolled in the study and approached family/whānau members to ensure that as a family/whānau they were happy with my presence in the bed space during this often distressing time. Family appeared to be comforted by the thought of having a friendly face in the bed space and often commented, once they knew I was an ICU nurse from the unit, that I would be an extra pair of eyes looking out for their family member. These perceptions, that I was 'overseeing care', were addressed with the family/ whānau where I reiterated my role as a researcher within the bed space.

The sample strategy used in this study was purposive sampling which relies on the researcher using knowledge of the population to carefully select participants who are typical of the population of interest (Gillis \& Jackson, 2002). Although, I had no influence on which staff cared for fat patients I was mindful of how my selection of staff to be interviewed could potentially misrepresent the group. During the interview selection process I was mindful to include staff who were male, female, fat, thin, young, old, and held junior and senior roles and that this needed to be proportional to the demographics of the group. Thus, the majority of the nursing interviews involved female junior nursing staff, whereas the doctor interviews involved mainly male doctors.

\subsection{Data collection}

Over a period of four months, between 2009 and 2010, commonly accepted fieldwork techniques were employed to collect cultural data from numerous sources. These methods of data collection were participant observation, ethnographic 
interviews and review of cultural documents and artefacts. There were 67 nurses, 13 doctors and seven patients who participated in the study. Of the nursing staff, 25 nurses were observed in practice and interviewed, 28 nurses were observed in practice only, and 14 nurses were interviewed only. Of the medical team, all 13 doctors were observed in practice in which five of them were subsequently interviewed.

I completed 21 days of patient observation during which I observed 53 nurses and 13 doctors providing direct patient care to seven patient participants. I interviewed 39 nurses and five doctors using a semi-structured interview format amounting to over 30 hours of audiotape (see Appendix 8). I obtained 12 cultural documents and recorded field notes on the use of material artefacts (see Table 3). The data collected provided information on behaviours, interpersonal relationships, verbalised thoughts and feelings, power structures and written accounts of care provision.

Table 3. Datasets generated from the fieldwork study

\begin{tabular}{|l|l|}
\hline Datasets & Details \\
\hline Interviews (44) & 5 doctors \\
& 39 nurses \\
\hline Field notes (9) & $\begin{array}{l}\text { Participant observation files (7) } \\
\text { General observations (1) } \\
\text { Reflexive journaling (1) }\end{array}$ \\
\hline Cultural documents (12) & $\begin{array}{l}\text { Patient participant files (7) } \\
\bullet \quad \text { Medical notes }\end{array}$ \\
& $\begin{array}{l}\text { - Intensive care daily observation charts } \\
\\
\end{array}$ \\
& Policies (4) \\
& Family information (1) \\
\hline
\end{tabular}

I used a labeling system to identify the different types of datasets so that clarity of the source could be determined when presenting the data in the findings chapter. Participants were identified by a pseudonyms and role throughout the study. When individual data results were presented, the following format was used: name and role, data source, and location within source. Table 4 provides some examples of how this labeling system appears within the thesis. 
Table 4. Data labeling system within findings

\begin{tabular}{|l|l|}
\hline Sophie-nurse, interview, p.2 & $\begin{array}{l}\text { Pseudonym name and role, data source, } \\
\text { location within source }\end{array}$ \\
\hline Fetu-patient, field notes, p.8 & $\begin{array}{l}\text { Pseudonym name and role, data source, } \\
\text { location within source }\end{array}$ \\
\hline $\begin{array}{l}\text { Guideline for the care of the } \\
\text { bariatric patient, material artefact, } \\
\text { p. } 17\end{array}$ & $\begin{array}{l}\text { Name of document, data source, location } \\
\text { within source }\end{array}$ \\
\hline $\begin{array}{l}\text { General observation, field notes, } \\
\text { p.3 }\end{array}$ & Data source, location within source \\
\hline
\end{tabular}

The number of participants within qualitative research is usually small as the depth of information and variation in experiences is of most interest, therefore large numbers are not practical or beneficial (Hennink, Hutter, \& Bailey, 2011). What guides the process of determining if sufficient numbers of participants have been recruited, and specifically, in this case, the amount of observational data collected, is the qualitative principle of 'saturation' (Gillis \& Jackson, 2002). Saturation refers to the point in data collection where participants' descriptions become repetitive and confirm what others before them have said (Gillis \& Jackson, 2002). Towards the end of the fieldwork both the staff participants' accounts of care during interviews and observations of care became repetitive and I used this later stage of data collection to confirm my thinking about the culture. Thus, by the end of the four months I was confident that I had captured the phenomenon under study.

\subsubsection{Participant observation data}

As already identified, I adopted an 'observer as participant' role (Gold, 1958), which allowed me to participate in the functioning of the group under investigation while recording information within the contexts, structures, and symbols that were relevant to group members (Gobo, 2008). Observations of staff occurred in all clinically designated areas of the unit, and the staff room and seminar room where nursing and medical handovers were conducted. Observational periods lasted approximately 12-16 hours per day, whilst a patient was enrolled in the study, and amounted to a total of 167 hours. Day shift observations started at $06.30 \mathrm{hrs}$ and usually finished at $22.30 \mathrm{hrs}$ following the evening medical rounds. For night shift observation. I started at $18.30 \mathrm{hrs}$ and continued until $07.30 \mathrm{hrs}$ the following morning (see Table 5). Although observations were made on weekdays and weekends, both 
day and night, the majority of the observations occurred during weekday daytime hours when more hospital services and medical teams were working (see Table 5). However, the combination of observational shifts allowed for a comprehensive view of patient care across a 24 hour, seven day timeframe in order to capture a rich perspective of the culture of care of fat patients. To maintain my concentration during these intensive and long observational sessions I took frequent 10-15 minute 'comfort breaks' (McCrea, Wright, \& Murphy-Black, 1998). The timing of these breaks were guided by my insider knowledge of the daily routines of the unit.

Table 5. Record of patient focused participant observation hours

\begin{tabular}{|c|c|c|}
\hline Participant & Observations periods & $\begin{array}{l}\text { Total hours of } \\
\text { observation }\end{array}$ \\
\hline Agnes & $\begin{array}{l}\text { Day } 10645-1300 \mathrm{hrs} \& 1830-2230 \mathrm{hrs} \\
\text { Day } 20630-1300 \mathrm{hrs} \& 1830-2200 \mathrm{hrs} \\
\text { Day } 31830-2230 \mathrm{hrs} \\
\text { Day } 4 \text { Nil observation } \\
\text { Day } 51530-2230 \mathrm{hrs} \\
\text { Day } 60630-1200 \mathrm{hrs} \text { (discharged) }\end{array}$ & 37 hours \\
\hline Rawiri & $\begin{array}{l}\text { Day } 11200-2200 \mathrm{hrs} \\
\text { Day } 20630-1530 \mathrm{hrs} \\
\text { Day } 30630-1530 \mathrm{hrs} \text { (discharged) }\end{array}$ & 28 hours \\
\hline Chris & 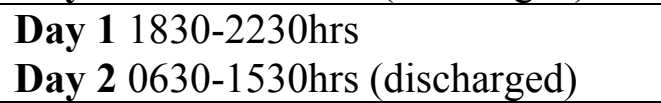 & 13 hours \\
\hline Don & $\begin{array}{l}\text { Day } 11330-2200 \mathrm{hrs} \\
\text { Day } 20630-1045 \mathrm{hrs} \text { (discharged) }\end{array}$ & $12 \frac{1}{4}$ hours \\
\hline Emiri & $\begin{array}{l}\text { Day } 11830-2230 \mathrm{hrs} \\
\text { Day } 20630-2130 \mathrm{hrs} \\
\text { Day } 30630-1030 \mathrm{hrs} \text { (discharged) }\end{array}$ & 23 hours \\
\hline Fetu & $\begin{array}{l}\text { Day } 11830-0930 \mathrm{hrs} \\
\text { Day } 2 \text { (Discharged) }\end{array}$ & 15 hours \\
\hline Gary & $\begin{array}{l}\text { Day } 11200-1645 \mathrm{hrs} \& 2100-2230 \mathrm{hrs} \\
\text { Day } 20630-1800 \mathrm{hrs} \\
\text { Day } 30630-1930 \mathrm{hrs} \\
\text { Day } 40630-1200 \mathrm{hrs} \\
\text { Day } 50630-1230 \mathrm{hrs} \text { (discharged) }\end{array}$ & $383 / 4$ hours \\
\hline
\end{tabular}

The primary focus of my observations was the human processes that directly related to the care of fat patients. However, to capture the wider influence of society on the culture of the unit, observations were not limited solely to the interactions between the patient and nurse or doctor. Instead, observations of all aspects of intensive care nursing were observed to truly grasp the social processes of this group. 
To achieve a real sense of how staff acted and behaved in front of patients and in the more relaxed non-clinical environments of the offices and staff room spaces of the unit I observed staff during meal breaks and in non-clinical areas of the unit. The staff room provided a rich source of information on the behaviours of nursing staff and the other healthcare professionals when engaging in how the external influences (such as the media), in regards to fatness affected staff behaviour. I engaged and immersed myself in the social rituals of the staff whilst taking every available opportunity to observe and record the group.

\subsubsection{Field notes}

I used field note journaling extensively to record my observations and thoughts about the study setting and participants during data collection. Field notes are the processes of 'raw' writing that provide the ethnographer with an accumulating written record of the observations and experiences of the culture (Condell, 2008; Emerson, Fretz, Shaw, 1995) . Writing up field notes as soon as possible is considered one of the key responsibilities of fieldwork, as memories fade fast and unwritten observations become superceded by subsequent events (Emerson et al., 1995; Fetterman, 2010). Equally, too long a delay in writing field notes affects the richness of concurrent notes (Fetterman, 2010). Field notes were completed at the time of observation and at the end of each observation shift reviewed, using reflexive practices. There were times, due to the length of observational days, that the tidy up of field notes and reflexive journaling did not occur, however, I made a conscious effort to always complete field notes before collecting more observational data.

Initially, brief field notes, or 'jottings', in the form of memory triggers were written during actual observation and expanded upon at the end of each shift. Jottings contained phrases, or key sections of conversation, timings of interventions, types of behaviours or activities, numbers of staff present, and anything that would aid my memory when expanding on the field notes later. As I became more skilled and familiar with undertaking observations and the detail in which they must be recorded, my field notes became extensive, only requiring reflection and minor additions of information after the observation period. These handwritten journals were then manually written up as electronic documents in preparation for data analysis. The 
detail contained within these records have been identified as paramount in discerning what participants take for granted as routine and thus less likely to raise in discussions when questioned (Silverman, 2013). I used field notes to describe the events, people, conversations and my feelings regarding the care provided to study patients. The notes detailed all aspects of contact with the ICU from arriving to leaving at the end of observation. Additionally, brief field notes were made immediately following interviews about my thoughts in regards to the interview discussions and non-verbal behaviours of the participants. These were later added to the interview transcripts, in the form of annotations, to provide contextualisation to enrich the data (see Table 6).

Table 6: Examples of annotated interview transcripts

\begin{tabular}{|c|c|}
\hline Interview transcripts & Annotations \\
\hline $\begin{array}{l}\text { "I would be concerned if someone I knew was } \\
\text { really big you know and for example I know that } \\
\text { this is confidential. Someone like [name omitted] } \\
\text { I would love [name omitted] to lose some weight } \\
\text { and people have tried because they're concerned } \\
\text { that she's going to fall over, we're going to have } \\
\text { to do CPR or something like that" (George-nurse, } \\
\text { interview, p.12) }\end{array}$ & $\begin{array}{l}\text { George lowers his voice, glances around the } \\
\text { empty interview room, and leans in close to the } \\
\text { microphone so that no one can hear what he is } \\
\text { saying. }\end{array}$ \\
\hline "I've seen several fat large people die" & $\begin{array}{l}\text { On saying the word 'fat' David immediately looks } \\
\text { up to catch my eye to see if I had heard him, and } \\
\text { quickly changes his vocabulary to 'large' people. } \\
\text { A tiny smile creeps across his face almost in } \\
\text { embarrassment that he had let the word slip out. }\end{array}$ \\
\hline
\end{tabular}

\subsubsection{Interviews}

Whilst ethnography focuses on observation as its main method, interviews do provide an important contribution to understanding the participants' perspectives of their lived experience (Fetterman, 2010). Fundamental to this research was understanding the staff perspectives of their experiences of caring for critically ill fat patients. Thus, interviews were used to capture feelings, thoughts and intentions of staff when interacting with fat patients, which would not have been possible from observation alone (Patton, 1990). The process of listening, Forsey (2010) suggests, is at least as significant as observation, as "ethnographers are more participant listener than observer" (p. 561). If conducted with "ethnographic imaginary", engaged listening during interviews can be equally effective in capturing the cultural context of the group's lived experiences (Forsey, 2010, p. 567). Interview data collected during the study was considered to be an important data source to that obtained by participant 
observation, as the interviews enriched the understandings of the situations and behaviours observed in practice (Gobo, 2008). However, during data collection, participant observation was always prioritised over interviews due to the more limited opportunities available to observe patient care practices, thereby, preserving a focus on observation, as the primary data collection method of ethnography.

Focused ethnographic interviews are usually highly structured around the specific issues of the research problem (Higginbottom et al., 2013). For this reason, I chose to develop a semi-structured interview format (see Appendix 9) to conduct the interviews, which were developed using both the Patton (1990) and Spradley (1979) frameworks for ethnographic questioning. Both frameworks are frequently cited resources for preparing ethnographic interviews in comtemporary ethnographies (Coombs, 2004; Madison, 2005). These frameworks were used to structure the language used in interviews to elicit credible ethnographic data, although the original research question and observations in the field guided the interview content. The initial interview questions were informed by the literature and my own experiences of working as an ICU nurse caring for fat patients.

The initial interviews focused on three areas: nurses' and doctors' experiences of caring for fat patients in the study unit and in prior employment; personal thoughts and beliefs about fatness as influenced by society; and resources and education opportunities for nurses and doctors in the care and management of fat patients (see Appendix 9). Information was elicited using example questions, explanation questions, contrast questions, opinion or value questions, feeling questions, quotation questions and descriptive grand tour questions (see Appendix 9). Descriptive grand tour questions are a type of ethnographic questioning that explores a scene which may have many and varied elements of the culture identified, such as that which occur when asked to describe a working day (Spradley, 1979). I used a funneling technique in the interview which involved using broad generalised open ended questions to explore topics and then 'funneling down' to more specific and closed questions (Kvale \& Brinkman, 2008). 
As the study progressed, and my skills and confidence at interviewing improved, I was able to incorporate specific questions in regards to the rich points of social interaction observed in the field. Through addressing the gaps in my own comprehension of the phenomenon observed in the interview, I was able to develop new understandings of the study group. Thus, the latter interviews were specifically used as a means to clarify certain practices, beliefs and values that would support my interpretations of the observations of care.

Formal interviews were conducted during staff participant work hours in one of two interview rooms on the unit. An interview appointment was made with each participant willing to be interviewed and occurred following the evening medical ward round, when the unit was usually at its quietest. Occasionally interviews were rescheduled to a different day if the unit was particularly busy. All interviews were audio taped and transcribed in their entirety by either myself or through a confidential transcription service.

In addition to the formal semi-structured interview, which had a specific preplanned agenda, many informal interviews, triggered by participant conversations and observation of practice were conducted. These informal interviews were primarily ad hoc, brief, casual conversations, and came about in two ways. The first were instigated by myself when I wanted to gain a deeper insight and clarity into the observations of practice of the participants. The second were instigated by the participants who either wanted to inform me of something which they thought was important for me to know or emerged from comments occurring in casual conversation that generated further questions to be explored. On both occasions, and due to the unstructured nature of the interviews, it was impossible to audio tape these interviews. Primarily, this was because I did not want to lose the spontaneity and genuineness of the information by disrupting the flow of conversation by reaching for the audio recorder. Therefore I made the decision to record the key points of the conversation in my fieldwork journal and expand on these notes later in the day or after the observation period had finished for that shift. In undertaking such ad hoc informal conversations I was mindful of whether participants were making a conscious decision to disclose information about themselves, similar to the formal 
interview setting, or just oblivious to the fact that I was delving for further insight into the social group. Therefore, in order to maintain a trusting relationship with my participants it was important that it became transparent that the informal interview would be used as data, after a short period of time in the conversation.

\subsubsection{Cultural documents and material artefacts}

Social reality is constructed not only in spoken language but equally in written texts. Cultural documents and material artefacts are an integral part of the daily activities of the social group and are a valuable source of ethnographic data (Fetterman, 2010). They can provide rich information about the context of the study setting and the wider organisation within which the social group exists (Hammersley \& Atkinson, 2007). Intensive care services produce many specialty specific documents, such as policies, guidelines, and manuals that govern and inform clinical practice. These written text do not just describe the organisations that produce them but play an important role in their construction (Silverman, 2013). These documentary sources construct facts, records, decisions and rules that are instrumental in the social routine and functioning of the group. Within healthcare, these documents construct a 'documentary reality' and are regarded as being objective, factual statements of reality (Hammersley \& Atkinson, 2007). Examination of these documents was an important process in understanding how written text influenced the clinical practices of staff being observed. Therefore I reviewed a range of cultural documents, including formal organisational and unit documents such as policies, guidelines, ICU orientation manuals, staff communications, medical and nursing charts, and patient medical notes.

The document data-set informed the interpretation of the data as a whole and its contribution to understanding how ICU staff cared for fat patients has been embedded within the narrative of the findings chapter. For example, the bariatric policy provided evidenced-based information on the physiological changes caused by fat and provided staff with instructions on specific manual handling and positioning practices, and types of resources available. These documents played an important role in the construction of the social routines of moving and positioning fat patients during care and are therefore reported on during the observation of staff as they engaged in the manual handling practices. Likewise, how staff responded to written words within 
cultural documents, such as medical and nursing notes, were recorded and presented as part of reporting on the findings.

Material artefacts, such as the specialised medical and nursing equipment used in the care of fat patients, often provide the focus for enactment of routines and rituals where manipulation of objects are central to the observations of practice being made (Hammersley \& Atkinson, 2007). Therefore I recorded data about the use of material artefacts in my field work journal, detailing descriptions of their use, purpose and social meaning. An example of this is the use of extra large sliding sheets for all fat patients when repositioning the patient in bed, to reduce manual handling risks. This demonstrated the social importance afforded to maintaining safe clinical practices for patients and staff when engaging in care.

Additionally, the environment and physical space in which the social group functions can have significant influence on the shaping of cultural interactions, rituals and routines observed in ICU. The bedside nursing desk provides the focal point for many interactions between participants, in particular the ward round, where decisions are made about patient care and documentary records produced. The examination of the physical space allows for an understanding of how the environment influences activity, how a sense of place, for example the hospital bed space, is reflected in individual and collective identities, and how places are used by the social group to construct realities (Hammersley \& Atkinson, 2007). Typically, the ICU physical layout is spacially arranged to: contain patients and visitors within certain areas of the unit; provide maximum surveillance of patients by staff; segregate clinical work from non-clinical work; and increase the efficiency of nursing activity. Activities are therefore constrained to certain ways of functioning within specific areas of the unit, which in turn construct the ICU culture. The positioning of the patient's bed in relation to the nursing desk used for recording and documenting patient care provides an example of how the physical layout constrains social activity. I recorded all of these considerations in my field notes which provided the context for the narratives created about the social group. 
During the process of data collection and write up of the thesis, I conferred frequently with participants about their level of comfort with the data collected and sought on-going permission to use this data in the thesis. Participants were involved at all stages of data collection and staff were involved during the interpretation and write up stages of the findings. At the beginning of every period of participant observation, I reaffirmed that staff and patients gave permission for me to observe and record care. At the end of each observation period, I checked with participants whether there was anything that had occurred during the observations that they wanted excluding from the field note data. None of the participants requested that periods of observation be excluded from the findings.

Likewise, participants were given numerous opportunities to be involved in decisions regarding interview data that would be used in the findings. At the end of the interview, participants were asked if any aspects of the interview had made them feel uncomfortable and if so, whether they would like that part of the interview removed from the data. Additionally, participants were offered a copy of their interview transcripts for review and editing. None of the participants asked for sections of their interview to be removed. The general response by staff was that they had been honest in the interviews about their feelings and experiences so did not mind it being used in the thesis.

During data analysis, I conferred with staff who featured significantly in the findings to reaffirm permission for use of data in the thesis. During these conversations I discussed how this data would be contextualised within the study findings. These conversations were mutually beneficial as it allowed staff the opportunity to be fully involved in the write up and increase their involvement in the study and have ownership of the culture represented in the findings by way of 'member checking' (see Section 4.18).

\subsection{Data management and audit trail}

Audit trails are used in qualitative research to review and examine the research process by documenting all the raw data generated, as well as the methods and sources of data generation and analysis decisions (Gillis \& Jackson, 2002). It 
provides evidence to support the rigour and trustworthiness of the study (Shenton, 2004). Due to the immersive nature of the study method, providing robust audit trail information was paramount. Particularly, because I was immersed in the data while still generating data, where perceptions were being continually formed, which could have influenced the future foci of the data collection and subsequent direction of the study. Therefore I created three audit trail documents as part of the data management and analysis strategies used to establish the trustworthiness of the interpretations made of the research data. These documents were a data management spreadsheet, a research journal, and a coding log. Each document provided evidence for supporting the claims made and how the findings were generated from the data (Richards, 2009).

The data management spreadsheet was created to systematically record the handling of each individual piece of data from the moment it was collected through every stage of analysis. The primary function of this spreadsheet was to record the dates and key processes that occurred during the management of the data, such as transcription accuracy checks, the creation of participant narrative profiles and annotations, and raw data and thematic coding. The dates of each of these stages in data management and analysis were recorded within this spreadsheet which acted as a checklist for systematic processing of data (see Appendix 10).

The research journal was created at the inception of the research study and was used to capture initial ideas, thoughts, assumptions and preconceived ideas and directions of the study including methodological possibilities and ethical concerns. However, thoughts and ideas about the study direction during fieldwork were made in my fieldwork journal and was specifically identified and recorded as fieldwork data. This was because the ideas generated at that time were in direct response to participant observations which guided the fieldwork focus and influenced the nature of data collected. Thus, fieldwork ideas were considered to have been generated from within the data and treated as data (Richards, 2009). In contrast, ideas and thoughts occurring during data analysis were considered to have been generated about the data. Therefore, following data collection, any thought or decisions made about the data and study were subsequently recorded in the research journal and became audit trail information. 
Coding logs were created during the coding phase of data analysis to provide a chronological record of the generation of the codes and detail information on the operational definition of data codes. The coding log documented the date a new code was created or modified, the name of the code, and a description of the meaning of that code (see Appendix 11). This process was an important part of the analysis and audit trail process as they provided clarity about what the code meant when it was initially created. Additionally, it provided instructions about what data could or could not be included within this code at later stages of the analysis. For example, the code 'nursing challenges to care' provided instruction when coding that only words and phrases that identified or described challenges encountered by nurses when caring for fat patients, could be reposited in this code (see Appendix 11). This log was recorded in my research journal in addition to the automated coding log that could be generated by the data management software (NVivo 9) which was used as the repository for all the research data. NVivo 9 computer software was used as a tool to facilitate the management of data during analysis and provide an audit trail of coding decisions.

\subsection{Data analysis}

For ethnographers, data analysis begins from the initiation of the research study to the final words written in the ethnographic narratives. Throughout this process the ethnographer must make choices between the logical and alluring routes of discovery, between the convincing and unfounded yet fascinating data, and between genuine patterns of behaviours and a series of apparently similar but distinct reactions to social interaction (Fetterman, 2010). All of these decisions are both datagenerating and analytical as they come from within the data and are made about the data, respectively. In making such choices, the ethnographer must be attentive to both detail and the larger context, as decisions made early in the field can influence the claims made about the social group under study. Therefore, "analysis in ethnography is as much a test of the ethnographer as it is a test of the data" (Fetterman, 2010, p. 92). When confronted with a rich collection of data the ethnographer's ability to think and to process information meaningfully and usefully is tested. 
Preliminary analysis began with the preparation of data and included accuracy checks of the interviews against audiotapes and adding annotations to the transcripts that captured vocal intonations and gestures, and transcribing my field note journal into electronic documents. During this time I wrote simple key words and thoughts about the data in my research journal as they were occurring during this tidying and organising process. Annotations of the raw data had two purposes; the first to record vocal intonations, and gestures/ body language observed at the time the data was collected which would add contextualisation to the data during analysis; and to ask questions of the data for further follow up later in the analysis (Richards, 2009).

The analysis of the data was conducted in three stages in which different types of codes were generated and used to inform the conceptualisations of the culture studied. The first stage of analysis was 'deconstruction' where the data was systematically broken down into distinct concepts (Strauss \& Corbin, 1990). This stage of data analysis is often referred to as "first order" (Van Maanen, 1979, p. 39) or “open coding" (Gobo, 2008, p. 227), as it is an organisation of the 'facts' of the data from the participants' perspectives. The main objective of this stage is to deconstruct the events and actions into a series of concepts which allows for new ways of making sense of the data (Gobo, 2008).

Deconstruction of the data was undertaken using NVivo 9 and occurred in two phases. Firstly, I created topic codes ${ }^{30}$ of raw data to store large amounts of information on a particular topic. These codes were useful in allowing the contextualisation of the information to be retained and for various ideas in relation to the topic to be viewed across the social group. For example, "nursing challenges to care' was one topic code which allowed for collation of significant volumes of data whilst at the same time differentiating data from that which referred to 'medical challenges to care'; thus maintaining data contextualisation. The second phase was the deconstruction of events and actions contained within each topic code, where the raw data was further coded, identifying key words and phrases repeatedly used. For example, the following codes were created under 'nursing challenges to care':

\footnotetext{
${ }^{30}$ NVivo uses the term 'nodes' instead of 'codes' to refer to the place where information is stored regarding the code (Richards, 2009).
} 
'equipment'; 'positioning'; 'mobilisation'; and 'patient size'. Words and phrases that identified or described how the patient's size was problematic in nurses conducting care was coded in 'patient size' (see Appendix 12). All data, such as interviews, chart reviews, and observations, were coded in this way, before cross-referencing of information occurred about a participant across the data sources at later stages of analysis.

The second stage of analysis was 'construction', where the concepts previously developed were reassembled into a new pattern of thought to explain the facts of the data (Strauss \& Corbin, 1990). These codes are often referred to as "second-order" concepts (Van Maanen, 1979, p.39) or "axial coding" (Gobo, 2008, p. 234), as they are themes and conceptualisations produced by the researcher to explain patterns observed in the first-order concepts. These codes expressed new ways of seeing the data and conceptualising the findings of the research (see Appendix 13).

Construction of the data into themes and conceptualisations occurred in two phases. The first phase was initially undertaken in NVivo 9 where I examined the first-order concepts looking for similarities which could be combined as one theme which I then renamed. For example, the first order concepts of 'improvisation' and 'forcing patients to fit' were merged together and renamed as 'making patients fit' (see Apendix 13). This process of analysis was continued with the rest of the data codes. The second phase of construction was a repeated exercise of the first phase, only this time focusing on the new themes created in phase one, which enabled a progressively narrower focus to two main conceptualisations of the data (see Appendix 13). At this point in the analysis I found using NVivo 9 was too limiting in its ability to allow me to move and play with ideas to construct a story of the social phenomenon observed. Therefore I resorted back to writing in my research journal and constructing ideas on large pieces of paper. Throughout this, and the subsequent stage of data analysis, I used a constant comparison technique to compare incidents applicable to each theme that emerged from the data (Glaser \& Strauss, 1967).

The third stage of analysis was 'confirmation' where the conceptualisation of the central phenomenon was constructed into a short story or descriptive narrative 
using the second-order concepts (Strauss \& Corbin, 1990). This narrative has been presented within the findings chapter as an overview of the conceptualisation of fat patients as 'misfits' (see Section 5.3). This stage is often referred to as "selective coding" (Gobo, 2008, p. 235) as the focus is on confirming or refuting the 'conceptualised story' by reanalysing the data for factual confirmation. It was during this stage that the works of Goffman $(1959,1963)$ and Hochschild $(1983)$ became apparent in understanding the social phenomenon of how fat patients were percieved and cared for in ICU.

In the process of analysing the data and conceptualising the social phenomenon, participant profile narratives were created. These documents recorded all my thoughts and feelings about each individual participant and was used to build a profile of their attributes, personalities and opinions about the care of fat patients, and as a means of comparing data sources about that individual. For example, many participants were interviewed and observed in practice. These interviews and field notes were coded separately, prior to comparisons being made for consistency across sources. This cross-referencing of information was recorded in the participant's profile narratives and were of particular value during the confirmation stage of analysis.

\subsection{Protecting the identities of the study participants}

New Zealand has a small population and maintaining anonymity is often a significant issue for researchers. This is especially true when researching small communities, such as intensive care, and when using detailed descriptions of the people and settings to illustrate and report findings. Confidentiality and anonymity issues were addressed, in relation to the study site and the individual participants, at the outset of any research (Houghton et al., 2010). Addressing site and participant confidentiality was a constant consideration throughout the study process to ensure that whilst removing all identifying features of the participants and site, the research findings presented still accurately reflected the social phenomenon under study.

Maintaining the anonymity of the study site has been of continued concern, given my connection and chosen research design. It was not conceivable to ignore my 
relationship to the study site or participants as a way to manage this issue due to the methodological underpinnings of the study, which required explicit articulation of my relationships with the field. Although the study site would never be formally identified or confirmed, my position within the ICU community would not make it difficult for others to ascertain. This particular issue was raised with the unit staff and was considered not to be a matter of concern for them. Lathlean (1996) suggests, that in such situations, we should acknowledge that real anonymity is a myth and be open about the setting. Although I have never confirmed the study site, many of the staff participants not only speak openly about the research site but also confirm their participation to others. This suggests that participants do not always want to remain anonymous and see no reason why that should be the case (Lathlean, 1996). In acknowledging that the study site may be revealed, protecting the identities of individual participants was of upmost importance to me.

The main concerns about participant confidentiality were identifying participants to other staff members on the unit, and to the wider ICU community by the very nature of their position held. Steps were taken throughout the entire project to minimise these risks, especially during data collection and analysis, and will continue during the dissemination of findings. During data collection I was the only person to have access to the raw data. My field note journal was kept on my person at all times and no real names were used in the journal. Participants were immediately assigned an alphanumeric code which was subsequently used for writing all field notes. Upon completion of data collection these alphanumeric codes were changed to pseudonyms. The participants' real identity and alphanumeric codes were stored in separate documents to the participants' alphanumeric code and pseudonym files. Both documents were needed in order to identify participants. These electronic documents were password protected and stored in separate electronic folders. The coding of staff to raw data was kept in separate places within my home and contained on different electronic folders. Only interviews were processed by a transcriber, frequently used by the university, who had signed a confidentiality agreement. My supervisors reviewed samples of my data once pseudonyms were inserted as part of supervising the data analysis process. All participants were informed of this during the consent process. 
Within the findings, as a way to protect prominent staff members, I chose to only identify nurses as having either senior nurse or staff nurse positions. The senior nurse was someone who has been employed within the description of a senior role position within the organisation and could be one of the following: Charge Nurse Manager, Associate Charge Nurse Manager, Clinical Nurse Specialist, and Clinical Nurse Educator, all of whom were formally part of the senior nurse structure within the unit culture. The staff nurse was someone who was employed on the unit to undertake the role of direct patient care. Due to the small medical team, I chose only to identify the medical team as doctors. Ongoing informed consent was sought from participants where I was concerned that others may identify the person in the research. In this instance, I met with that participant to reaffirm their level of comfort with the information being used. I am confident that this due consideration to the anonymity of the participants has produced sufficient anonymity without distorting the findings or misrepresenting the culture of the study group.

The time lapse from the data collection to publication helps to protect the anonymity of participants or at the very least make identification more difficult (Wolcott, 1973). Over time participants forget what others have said or indeed what they themselves have said. Issues that were critical to the unit and participants during the study have lost their urgency and identification over time. The continued turnover of staff, characteristic of hospitals, considerably contributes to protecting the anonymity by merging together the personalities of new patients and staff with old ones. The only constant connection to the study site is myself.

\subsection{Rigour and trustworthiness of the research process}

A criticism of ethnography, as a qualitative research approach, is the issue of validity and reliability of the data (Hammersley \& Atkinson, 2007; Seale \& Silverman, 1997). One of the main criticisms is that ethnographic data is derived from the observations of complex social interactions that become the researcher's subjective interpretations and perceptions of a culture, thus rendering it 'unscientific' (Hammersley, 1998). Equally, the issue of replicability is often debated when critiquing qualitative research approaches, where it is implied that if the research is 
not replicable then it is invalid and unreliable (Atkinson \& Silverman, 1997). These challenges of establishing the trustworthiness of qualitative research have been debated for many years resulting in the development of numerous frameworks and strategies to support this process (Shenton, 2004). Ethnographic researchers must demonstrate that their constructed portrayals of the culture the emic perspectives are true by making evident how those portrayals were constructed. The framework developed by Guba (1981), for assessing the rigour of qualitative research, has been used to consider the trustworthiness and rigour of this study. This framework addresses the following areas of trustworthiness: credibility, transferability, dependability, and confirmability.

\section{Credibility}

Credibility, the most important factor in establishing trustworthiness, refers to how accurate the phenomenon under scrutiny has been recorded and presented (Lincoln \& Guba, 1985; Shenton, 2004). This study has aimed to attend to the issue of credibility in many ways. The first was by adopting a method and sample that would capture the culture and behaviours of the social group, which were informed by and derived from previous comparable studies (Shenton, 2004; Thomson, 2011). The process requires an ongoing review of the methods and sample being used, to ensure that the culture under investigation is being correctly captured. At the outset of data collection, the initial study sample was fat patients and nurses, however it soon became apparent that a change to the sample population was needed to include all healthcare professionals, in order to truly record the culture of the unit.

Credibility can be further strengthened by the familiarisation with the culture of the participants before the data collection commences, thereby increasing the 'prolonged engagement' with the participants (Lincoln \& Guba, 1985; Shenton, 2004). Although I had seven years previous engagement with the unit, prior to data collection, it was important to recognise the distinction between being a nurse and then a researcher. As a nurse I had familiarity with the unit functioning and already established trusting relationships, however, I had not observed the practices of ICU with the critical eye of a researcher and therefore, I would be viewing the same familiar environment from a different perspective. However, familiarisation as a result 
of my previous nursing role had the potential to effect my professional judgements and ways of seeing aspects of the culture (Lincoln \& Guba, 1985; Shenton, 2004; Silverman, 2013). The concerns of familiarisation as a nurse have been addressed within this study through the process of reflexivity, a process that further strengthens the credibility of the research (Shenton, 2004), and is discussed later in this chapter.

Triangulation provides a means of enhancing credibility by cross-checking different data sources to determine if they substantiate one another (Walsh, 1998). By using different methods, each compensate for their individual limitations whilst exploiting their respective strengths (Guba, 1981). Different data collection methods, informants and sites often form the basis of triangulation (Shenton, 2004; Silverman, 2013). This study used triangulation of data collection methods and informants. By using multiple methods, it has been suggested that an increased understanding of the phenomenon occurs leading to a more comprehensive representation (Fetterman, 2010).

Equally, triangulation during data analysis allows for one source to be tested against another, which can lead to a true understanding of the situation (Fetterman, 2010). For example, triangulation was used in the analysis of the data to compare the individual participant interview data with field notes of their care provision. Primarily this was to determine if what a participant said during their interview was reflected in the observations of their practice. These comparisons not only assisted in crosschecking the data sources but supported the creation of participant profile narratives described earlier. Similarly, informants were triangulated against each other so that viewpoints and experiences could be verified against others and the attitudes, behaviours and perspectives of the group under scrutiny would be representative of a wide range of people (Shenton, 2004). By using a comparative method to examine these multiple sources of data and informants, provisional ideas could be tested (Silverman, 2013).

The continued relationship with the participants following data collection meant that 'member checks' (also known as 'informant feedback' or 'respondent validation') could be easily undertaken with participants and has been acknowledged 
as an important way to reinforce a study's credibility (Lincoln \& Guba, 1985; Richards, 2009; Walsh, 1998). There are many different ways that member checking can be used to support the credibility of the study. In this study, I used different member checking strategies during data collection, analysis and the write up of the thesis. During the data collection participants were regularly consulted on the accuracy of the data through informal interviews, where clarity of observations and meaning was sought. Additionally, my ongoing relationship meant that provisional ideas were at times discussed with participants during the analysis phase of the study so that they could offer reasons for particular behaviours observed during the fieldwork, correct any misinterpretations, and provide an opportunity for additional imformation to be volunteered. For example, I consulted with a participant on why particular decisions where made when blood pressure recording equipment did not fit the patient's arm. This strategy has been employed and recommended by many qualitative researchers (Lincoln \& Guba, 1985; Walsh, 1998).

This study collected data from a range of methods and informants, and engaged in a process of 'member checking' which allowed for differences and similarities to be examined during the coding and analysis phase. These methods provide more confidence that the findings are a credible representation of how nurses and doctors care for fat patients in ICU (Roper \& Shapira, 2000). However, they cannot claim to be representative of care within all ICU's in New Zealand, given that it was a single centre sample who may have differing ways of delivering care.

Like participant 'member checking' peer review of the study was regularly undertaken during $\mathrm{PhD}$ schools and when presenting at research seminars and conferences. Shenton (2004) suggests that the reviewers fresh perspectives provide valuable contributions to refining research methods, developing greater explanations of study designs and strengthening arguments based on their comments. For example, the feedback received from presenting at an ethnography conference on the the shifting boundaries of engagement' was an invaluable process in understanding how my nursing role within the unit may have influenced the study, as percieved by the audience (Hales, 2012). 
The final method by which credibility was attended too was the way 'thick description' of the phenomenon was observed, recorded and used (Shenton, 2004). Presenting detailed description of the actual situation and the context that surrounds them allows for the reader to determine if the overall findings reflect the phenomenon portrayed. Therefore, I used densely textured descriptions within the findings to support broad assertions about the culture in a way that allowed others to judge the rigour of the analytical process.

\section{Transferability}

The transferability of the research refers to the degree to which the findings could be applied to practices and experiences in other places (Merriam, 1998). The research findings of this study do not and cannot claim to be representative of nurses' and doctors' care of fat patients in other intensive care facilities. Since the findings of this study are based on one site it further limits the degree to which the findings can be generalised to other settings, due to the specific influences of the context (Silverman, 2013).

Identifying the value of the research findings to the wider audience is an important issue raised in qualitative research. In this type of research, transferability requires the 'sending context' to be presented by the researcher which is subsequently applied within the 'receiving context' of the reader (Shenton, 2004). Thus, researchers do not attempt to convince others of the inferences they can make to their own clinical situations. Instead researchers have the responsibility to ensure that there is enough contextual information regarding the fieldwork site and study methodology to enable the reader to make accurate inferences to their own situation (Firestone, 1993; Lincoln \& Guba, 1985). Thus, determining transferability to the wider research and clinical communities resides with the reader. The reader, having considered the contextual descriptions of the study, must determine how far they can confidently transfer the results and conclusions of the research to other situations (Shenton, 2004). This study has therefore aimed to provide sufficient contextual information and transparency of the methodology to support readers in determining its transferability. 


\section{Dependability}

Dependability addresses the issue of reliability in quantitative studies (Shenton, 2004). Reliability refers to the degree to which the same results would be obtained if the study was repeated within the same context, using the same methods and the same participants (Shenton, 2004). As culture is evolving over time it is unlikely that the findings of a qualitative study would produce the same results if replicated (Richards, 2009). Therefore, the aim is to provide enough detail regarding the study design to enable others to repeat the study, without necessarily focusing on gaining the same results (Shenton, 2004). Thus, the purpose of extensively documenting the research design was to enable others to apply a similar systematic approach to the gradual accumulation of knowledge about the culture of care of fat patients within ICU.

\section{Confirmability}

Confirmability refers to the extent to which the research has been influenced by the researcher (Guba, 1981). In qualitative research this is addressed through identifying the researcher's bias and by confirming the findings are the results of the experiences and practices of the participants and not the preferences of the researcher (Shenton, 2004). Thus, researchers have the important task of representing the participants perspectives in a credible and dependable way whilst acknowledging the influence of their presence in that representation. Researcher influence/ bias has been addressed in the study in four ways: triangulation, audit trail, admission of the researcher's beliefs and assumption, and reflexivity. Triangulation is advocated as a means of reducing researcher bias (Shenton, 2004). Audit trails assist to expose the researcher's influences throughout the research process by making evident the procedures and decisions made. A 'data orientated' approach was taken when documenting the audit trail which demonstrated how the data was gathered and processed in order to reach the conclusions of the study (Shenton, 2004).

The admission of the researcher's beliefs and assumptions are often addressed in the reflexive process of ethnographic research. Reflexivity facilitates a critical examination of how the researchers beliefs, assumptions and interactions during the course of the fieldwork influence the data and interpretation. Addressing the influences of my background, beliefs, and connection to the study site was an 
important aspect of the study. Given my work history and position within the study site it was inevitable that my preconceptions of the unit and prior relationships with the participants would influence the study. Therefore it was paramount that I was reflexive and transparent about these influences.

\subsection{The ethnographic journey of fieldwork}

Undertaking ethnography within one's own workplace poses many ethical, methodological and professional challenges to the study design and methods adopted within the study (Asselin, 2003; Simmons, 2007). These challenges included my ethical responsibility to the participants, the methodological concerns of my involvement within the research, the professional obligations and concerns of observing my colleagues in practice, and the willingness of my colleagues to participate in research that involved observation of their practice by me. Many of these challenges were explored through adopting a reflexive approach to the study. This permited the exploration of the ways in which I played a part in the study and embedded myself into the text. In this way the data was not collected by myself but made collaboratively from engaging with the participants (Richards, 2009). Thus, reflexive field note journaling, which I used extensively throughout the research process, provided data that recorded collaborative constructs, between myself and the participants of the study (Richards, 2009).

How I entered the field, conducted the fieldwork and left the field, as a researcher, to return as a nurse and colleague, was a strong focus of the reflexive discussion. In particular, I remained engaged with the unit and staff by staying deeply connected, but not central, to the group's activities. Additionally, the power, role change, and the new space that I occupied within the group was carefully considered to elicit how and to what extent my prior relationships, as a member of the social group being observed, affected the data collection. Through acknowledging my existence within the social group being studied I have made no attempt to remove the effects of my role in the research process. Instead like many ethnographers my intention has been to understand it (Hammersley \& Atkinson, 2007). 


\subsubsection{Entering the field and negotiating my researcher space}

Entering the field is always a time of great apprehension, as first impressions and actions of the researcher can significantly affect the acceptance into the group (Wolcott, 1995). An important advantage for me, in terms of acceptance by the ICU team and patient participants, was that I was an intensive care nurse from the unit. The nursing team in the study knew that my clinical skills were current, and having worked alongside and with them in practice this helped them to accept me as a nurse researcher. Equally, I felt that staff were comfortable and less anxious in my presence because they knew, like them, I was not perfect and they saw me as someone who knew how things 'really were'. Additionally, I had participated in the situations in which I was now about to observe, therefore I would not be judging them because I was one of them.

My relationship with the staff, when beginning the observation period, appeared unchanged, despite the frequent conversations about my new role on the unit. This seemed to strengthen the study, as nurses were relaxed about my presence but at the same time challenging for me to establish my position as a researcher. Nevertheless, it was important for me to always be strengthening the rapport with my colleagues, whilst at the same time moving myself towards the outer edges of the cultural group in which I had membership. In doing so, I never became an outsider looking in but remained always an insider exiled to the boundaries of my social group (Tuhiwai Smith, 2010). This inbetween space that the nurse researcher occupies, Walker (1997) described as the "the borderlands", where the nurse researcher "inhabits the 'slash' rather than the territory on either side" (p. 4). This metaphor aptly decribes the constant tensions that nurse researchers face when they hold dual identities in nursing ethnographies. For me, the space that I occupied appeared to be determined by the professional, ethical, and research situations of the field at the time of observation and the varied extent of my participation with the staff in my role as 'observer as participant'.

Finding and defining that space which I would occupy was difficult both for the participants and myself. At the beginning of the fieldwork this space was continually moving, expanding and decreasing as I negotiated my way through the 
role transition of a nurse becoming a researcher and with the engagement of different study participants. Likewise, the unit staff had to undergo a transition to accept our new and temporary working relationship. Like Cudmore's experiences (Cudmore \& Sondermeyer, 2007), I was never conducting the study from a static perspective, and therefore my aim became to always remain at a 'critical distance' from my colleagues.

In reality, this 'critical distance' was always being threatened by the potential role confusion that occurred with the adoption of the 'observer as participant' position. The boundaries of being a nurse and researcher were therefore much more blurry and indistinct, and often the space that I occupied became situational and defined by the perceptions of the study participants. The possibility for role confusion during data collection was considerable (Asselin, 2003; Simmons; 2007). For the ICU nurses it was difficult for them not to ask for assistance and advice as they still saw me as one of the team but just undertaking some non-clinical work, which is not uncommon for the unit. Nurses, in particular those new to the intensive care environment, would ask for advice about patient management or set up of equipment and how the nurse on the previous shift had done something so that they could be consistent. Patient participants often looked for my confirmation that the nursing care was of a high standard or asked me to repeat what the medical team had told them on the medical rounds.

During medical rounds, the consultants would ask me questions regarding the care in the previous 12 hours and in particular what another specialist from another department had decided. Some staff, who understood the process of research and more specifically ethnography, would say 'are you allowed to tell us?' This led to an ethical dilemma and tensions between withholding important information regarding a patient's care that no one else present could answer, and the research methods of not influencing practice. At times, it was difficult to refrain from commenting during medical rounds when nurses didn't relay information, and I found myself assessing the importance of the information before deciding to comment. The previous decision to act only in situations of 'immiment danger' seemed somewhat extreme and unreasonable when I was faced with these types of practice dilemmas. I resolved these issues by considering the impact my omissions would have on the patient and the 
research process. If I had been researching clinical decision-making then the impact of involving myself in the ward rounds would have been detrimental to the research process, as this was not my focus, I was guided by my duty of care. Additionally, I didn't want to appear obstructive and unhelpful as this would be potentially reciprocated within the field or on my return to work. These types of dilemmas illustrated that even for me the space that I occupied was a contentious one to which I was always re-evaluating.

Establishing reciprocal relationships with my colleagues as a researcher was an important part of the research process (Hammersley \& Atkinson, 2007). I was aware of all the time my colleagues had given to being part of the study and I wanted to reciprocate whenever possible, however I was mindful that this needed to be in ways that did not influence the field. In this respect, I needed to be a part of the team and yet excluded from all responsibility. I needed to be seen as a team player but without playing. There were times when I had to assist staff to validate the tacit reciprocal agreement that they were helping me and I needed to help them in return. I used my insider emic knowledge of the social group to support participants in their daily work, these were small actions that signaled a greater sense of partnership and collegiality. Examples of such support included assisting a nurse with a turn when it was busy, making the tea trolley in an evening, getting a piece of equipment from the store cupboard or a wound dressing in preparation for the dressing change. All this I considered to be an acceptable part of being a team player whilst excluded from all responsibility. This type of reciprocity has been identifed as an important part of establising trusting and mutually beneficial relationships within the field, as they can significantly effect the willingness of participant self-disclosure and involvement in the study (O'Reilly, 2009; Simmons, 2007).

Despite my intentions to remain in my researcher role for the duration of the fieldwork, there were situations when I was required, both professionally and ethically, to step in and support the care of patients and transition back into the role of a senior nurse. In doing so, I moved myself back into the central functioning of the group and took up my nurse identity. The excerpt below illustrates an example of such a shift: 
It was a very busy morning shift and the unit was understaffed due to early morning sickness calls. I was observing the care of Agnes (patient) when the unit emergency bells rang indicating that a patient in a nearby bed space required immediate medical and nursing support. The nurses around me stopped their tasks and either went to the bed space where assistance was required or took over the care of the adjacent patients. I was asked by the associate charge nurse manager (ACMN) to take over the care of the sickest patient on the unit whilst the emergency was being dealt with. Her priority was ensuring the safety of all patients during the emergency not my research. My initial response to the situation had been the same as the ACMN and I found myself assessing the safety of the other patients so that nurses could attend the emergency (Agnes-patient, field notes, p.2).

This situation raised an important question regarding my position on the unit and the knowledge the staff participants had about myself as a nurse. Staff used their own insider knowledge of myself to influence the care and management of their patients. If I had been at another hospital, where my clinical skills were unknown, I would not have been asked or expected to intervene in emergency situations or participate in care when the unit was short staffed. The decision to participate in these situations would have been instigated by myself. However, the staff knew me as well as I knew them and this at times influenced the space that I occupied as a nurse researcher.

The relationship that I developed with the staff in my new role as researcher and my presence in that role was an important area for reflexivity in terms of how I influenced the field (Asseslin, 2003). The effects of the researcher's presence during the observations of practice have been debated in the literature (Asselin, 2003; Hammersley \& Atkinson, 2007). Although, strategies can be adopted to reduce the researcher's influence they can only be partially eliminated (Gobo, 2008). I was always mindful of how I responded to the situations I was observing through the feedback in my body language and conversations. I was careful not to covertly signal to the participants my intentions, my inner thoughts, and beliefs, which in turn could have jeopardised the acceptance to continue observing the group. To be accepted in 
my new role as a researcher it was important not to display any signs of personal and professional approval or disapproval regarding unfolding events. My role as the ethnographer was to observe and record not judge or influence participants and thus remain peripheral to the social activities of the group (Wolcott, 1973).

Ethnography as a means of inquiry acknowledges that the very act of observing or focusing attention on a particular aspect of behaviour can sensitise and alter that behaviour. As the behaviour under scrutiny becomes a self-conscious act by the participant, it often becomes a cause of concern, apology, defense or self-ridicule (Wolcott, 1973). Despite my awareness of this phenomenon and my attempt to remain aloof about my observation interests whilst conducting the study, there were instances that induced participants to be more self conscious of their behaviour. This was particularly evident when certain aspects of their practice received more critical attention than others. The use of language to describe fat patients was one example of how the subconscious behaviours of staff became sensitive ones when questioned during the interviews on this topic. At times, this increased the personal drive by some of the staff to become self-aware and conscious about their behaviours or change their practice. Therefore, this behaviour change observed during the study may in part be both natural and as a result of focused observation where many participants entered into their own reflexive process.

\subsubsection{Leaving the field and re-negotiating my space as a nurse}

Leaving the field has received much less attention in the literature than entering the field and negotiating social relations (Gobo, 2008). In particular, there is a lack of focus on insider disengagement, in part because this process is seen more as an introspective transition from researcher back into the role of social member, rather than a calculated and rigourously negotiated methodological process (Labaree, 2002). This idea that the insider disengagement is only an introspective act has been challenged, since the knowledge acquired and new understanding of the culture during participant observation functions to transform the social reality of the researcher (Labaree, 2002). The insider researcher leaves the field with a broader sense of the social group's hidden complexities which may influence their practices when transitioning back to the social group. 
Returning to work as a nurse after the research was an exciting prospect for me, because I had learnt as much about myself as a nurse as I had about my participants during the observation period. My intention had always been to return to work in my previous senior nurse position. However, upon completion of the data collection, I took up a teaching position at the University and continued my clinical shifts as a staff nurse on the unit. This meant that I would still be undertaking the same amount of clinical work on the unit but no longer as a senior nurse, instead I was working as a staff nurse alongside my colleagues. Again, after some negotiation, transition to this role was relatively smooth. The rapport that I had built with my colleagues as a researcher strengthened my relationship upon my return to work as a nurse in my new role of staff nurse.

Given the sensitive nature of the study there was always the possibility that the staff involved would feel misrepresented or challenge the interpretations or conclusions of the research making my return to work a potentially unpleasant experience (Labaree, 2002). These risks were carefully negotiated with the staff participants, myself and my supervisors prior to the study commencing and throughout the research process to minimise such an event occurring.

\subsubsection{Power relations}

The power based relations between the researcher and the researched must not only be acknowledged but negotiated within the research process (Merriam et al., 2001). Each stage of the research process, from recruitment to publication of the study, influences the balance of power within the relationships (Karnieli-Miller, Strier, \& Pessach, 2009). In addition to the power relations that occur as part of the research process, I was cognisant that my senior nurse position, within the researcherresearched relationship, could influence the study. Of specific concern for me was how this position of seniority within the unit would impact on the recruitment of staff, the willingness to self-disclose personal and professional thoughts and feelings during interviews, and how I would represent the participants in the final written text. The role which I held prior to the study has been carefully considered to understand the impact that my relationships with the study participants had on the data collection. 


\subsection{Chapter summary}

Described in this chapter were the philosophical and methodological positionings of the study and detailed descriptions of the methods of data collection and analysis. Throughout the chapter, the ethical tensions between the dual identity of the nurse researcher was examined where I provided a critical and reflexive discussion of how my duality and nursing position within my own workplace may have influenced the research process. The nature of the insider-outsider dichotomy was explored where I identified that the space that I occupied within the field was not static but dynamic and determined by the situational needs of the field. I have explored the potential strengths and weaknesses of the research design, and my association with the study site and detailed the measures taken to ensure rigour and trustworthiness within the study. The following chapter presents the findings of the study where fat patients are conceptualised as 'misfits'. 


\section{CHAPTER FIVE: FINDINGS}

\subsection{Introduction}

The findings in this chapter present the 'situated' experiences of caring for critically ill fat patients from the perspectives of ICU staff. From this perspective fat patients were conceptualised as 'misfits' within the ICU environment. I begin with a description of the ICU setting and provide an overview of the social phenomenon that existed during care situations. Following this overview I present each theme in detail to illustrate how intensive care staff managed their private and public 'face' presentations when caring for fat patients. The findings highlight the complexity and social awkwardness that existed within interactions between staff and socially stigmatised fat patients.

\subsection{The unit}

The unit was an 18 bedded intensive care which was divided into three notably different regions. These were the visitors waiting room, the clinical practice area, and the non-clinical and adminstration area. Each designated area was sectioned off by staff operated security doors which allowed for strict monitoring of access to the unit by staff members. The main visitors waiting room connected to the furthest end of the unit. The physical space of the waiting room was no different to other waiting rooms with rows of chairs lining the outside walls, coffee tables strewn with magazines, coffee cups and food items, and a small kitchenette with a microwave and drinkmaking facilities. High above the fridge in the kitchenette there was a wall-mounted television. Above the access doors to the clinical area there was a camera which monitored the visitors' arrival and relayed the images to the staff inside. Attached to this main visitor room was one of the interview rooms used for ICU family meetings. This small room also doubled up as a rest area for families to sleep overnight when their family member had been a new admission or was dying. At times, when the unit was busy, visitors could be found spilling out into the main corridor of the hospital where they took up residence on the atrium sofas, often spending the night wrapped up in hospital blankets. The visitors' waiting room was always busy in the day due to the many visitation restrictions during nursing and medical handovers and ward rounds. Families were excluded from the unit between 0700-1030hrs, 1600-1700hrs, 
1900-2000hrs and 2100-2200hrs, and discouraged from visiting overnight if the patient was stable.

The physical layout of the clinical area was segregated into a clinical care zone which contained the bed spaces, and a staff designated support area. The individual bed spaces lined the four outside walls of the rectangular shaped unit. Each bed space was identical in layout which meant that staff could work in any of the 18 bed spaces and be familiar with that space. Being familiar with the environment, equipment and other staff were important to the delivery of safe and efficient care. Above every bed there had been a ceiling hoist installed which was capable of lifting up to $200 \mathrm{~kg}$. The ICU beds were fully motorised and could be manoeuvred into semichair positions, and the bedframe itself could be tilted to a 45 degree angle. The maximum weight capacity of these beds was $267 \mathrm{~kg}$. The bed itself, was positioned in the middle of the bed space allowing for walking room all round and easy access to the patient. On either side of the bed were ceiling mounted equipment pendulums which had the ability to be moved into different positions. On these pendulums were electrical sockets to power the equipment, oxygen, medical air and suctioning ports, hooks and bars to attach intravenous infusions bags and infusion pumps, and resuscitation equipment. A large patient monitoring screen hung down from the ceiling and was easily visible for staff. Along the outside edges of each bed space was a work space trolley containing essential monitoring, and intervention equipment, a nursing administration trolley, laundry skip, and a wall mounted computer. Each bed space was partially walled off from the next one to reduce noise and increase the privacy for the patients. Patients were therefore, unable to see other patients in the adjacent bed spaces. The patient's only view was the walls of the staff designed central area and the foot traffic of staff and visitors walking past.

Along one of the outside walls were four isolation rooms. These rooms differed from the other bed spaces in that they were fully enclosed rooms with a glass sliding door facing out into the corridor between the clinical area and staff support zone. These rooms could be accessed directly via the sliding glass door or if the patient required isolation precautions via a separate small ante-room which allowed for isolation precautions to be maintained. One of these rooms was also the designated 
bariatric room. This room had been structurally engineered to support a ceiling hoist that was capable of lifting up to $550 \mathrm{~kg}$. The room had been purpose-built to manage any medical emergency or critical illness in which a tertiary level III ICU may be required to treat. Therefore the room was equipped with additional fittings that supported life-saving therapies. This room was normally set up as a standard bed space using the standard ICU equipment. The specific bariatric equipment, such as the bed, commode, bariatric hoist slings, extra wide sliding sheets, and larger gowns, were stored in a central place in the hospital as they were used for all patient admissions and therefore, retrieved when needed. Like the standard ICU beds, the bariatric bed was fully motorised and could be manoeuvred into semi-chair positions, and the bedframe itself could be tilted to a 45 degree angle. The only difference was that it was significantly wider and had a maximum weight capacity of $500 \mathrm{~kg}$. As the majority of patients weighed under $200 \mathrm{~kg}$, most patients were managed out on the main three sides of the unit as this allowed for better organisation of staff resources.

The central area of the unit was a staff designated zone with clearly marked signs restricting visitor access. A permanent blue-grey line on the floor separated out the official patient bed space from this central area. At each end of this staffdesignated zone there was a nursing station with more monitors, and surveillance camera footage of the entrances to the unit. Emergency resuscitation trolleys and defibrillators were prominently positioned and sign-posted at each of these stations. Between the nursing stations was a row of head-height partitioning walls and glass rooms full of equipment, drugs, and computer/administration spaces for accessing medical records, viewing investigations and co-ordinating medical care.

The non-clinical and adminstration area was positioned off the main clinical area of the unit at the opposite end to the visitors room. This space contained multiple offices for adminstration, medical, and senior nursing staff, changing rooms, the staff room, and the seminar room. The large seminar room was adjacent to the staff room and it was in these two rooms that nursing and medical handovers occurred. All nursing handovers occurred in the seminar room due to the large numbers of staff on each shift, whereas medical handovers were conducted in the seminar room in the morning, and the staff room in the afternoon and at weekends. The staff room had a 
well equipped kitchenette with microwaves, toasters, coffee machine, dishwasher, drink-making facilities and two large fridge freezers for storing staff meals. There was a wall mounted television, tables and chairs for more formal eating, and sofas with coffee tables for relaxing. There was enough seating for up to 14 people at any one time which made the staff room a central place of ongoing activity. Along the window sill was a display of gratitude cards from families and student nurses, and stuck to the window notices regarding upcoming social activities. The staff room was the place that most people started and ended their shift.

\subsection{Conceptual overview of fat patients as 'Misfits'}

Fat patients were 'misfits' in the space of ICU. The term misfits is used here to describe how fat patients were the wrong shape and size and were unable to easily adjust to the environment or circumstances they were in, and were considered to be disturbingly different from others. These definitions aptly defined the position in which fat people were viewed by staff and often how they viewed themselves. Being a misfit meant that fat patients fitted badly or failed to fit at all and therefore 'did not belong'.

In keeping with the definition of a misfit, fat patients didn't fit physically, medically or socially. Fat patients were conceptualised as 'physical misfits' because their body size and shape was too large to fit much of the equipment used in ICU in a range of different ways and depending on the distribution of their body fat. The beds were too narrow, chairs and commodes too small, hoist slings too tight and stretchers too spindly to hold their body. Equally, equipment was often inappropriate, ill fitting, and even occasionally failed. This 'misfit' of size and design between the patient and the physical environment put limitations on the types of care available to the patient.

Fat patients were conceptualised as 'medical misfits' as their anatomical body changes (fatness) challenged conventional understandings and ways of practising medicine, which is framed predominantly around the 'normal' body. Their altered anatomy and physiology increased clinical risk for the patient. Large fat deposits obscured and distorted underlying anatomical structures making it difficult to locate key anatomical landmarks. Additionally, the altered physiology meant that fat 
patients had poor physiologic reserve to cope with acute illness and had multiple comorbidities, adding to the severity of illness and complexity of care. These factors affected the doctors' ability to assess, diagnose and treat the diseased or injured body using conventional medical practices. This potentially led to missed diagnoses, increased technicality of invasive procedures and increased risk of fat related complications.

'Social misfits' conceptualises how fat patients didn't socially fit within the ICU. Their behaviours and attitudes were deemed unsuitable and juxtaposed to the social ethos of health as they were seen as lazy, unmotivated people who led unhealthy lifestyles by eating too much and not exercising. There was a misfit of 'behaviours' between the fat person's 'percieved' and 'expected' social behaviours in regards to lifestyle choices. Fat patients were discussed by staff in ways that revealed social difference, disapproval and moral judgement. Social difference was demonstrated through comparative dialogues that referred to 'them' the fat patient/person and 'us' the normal weight person. Similarly, language was used that objectified 'them' to establish further social distance between the two groups. Furthermore, their fat physical body was socially differentiated from other bodies as it was seen as being imperfect, ugly and grotesque, and something undesirable to others. Fat patients were acutely aware of these differences and were embarrassed and apologetic about their bodies particularly when requesting assistance from staff.

'Judging fatness' underpinned the space that the fat body occupied and reinforced all decisions about how the fat body, as a physical and social being, was constructed and cared for within the ICU. The judgements of hospital staff, visitors, and patients about fat patients infiltrated all aspects of the culture of the ICU. Fat patients were judged both morally and clinically. Moral judgements were premised on perceptions that fatness was a result of personal failings and thus were voiced regularly in disapproving ways. In contrast clinical judgements were often pragmatic decisions about how to manage the physical size of the unwieldy fat body. However, many clinical judgements were often infused with moral discourse through the use of sarcasm and irony. Judgements were passed on regularly between staff during medical referrals between hospitals, and during medical and nursing handovers of the patient. 
'Contempt' was a way that staff expressed their moral judgement and disapproval. It involved the judgement that because of a moral or personal failing the fat person had compromised their social position with the other person based on a value or standard important to that person. In this case, the fat person was held in comtempt by the staff member for not being socially responsible for their health and wellbeing, implying that they themselves were taking this responsibility seriously. Contempt for the fat person involved a way of negatively and comparatively viewing or attending to that person, who had not met social standards deemed important to society. Staff were observed to be contemptuous towards fat patients. This appeared to occur as a result of the culture within the unit where social differences were reinforced through making negative comparisons, and criticising and condemning fat patients from a position of assumed moral superiority. Staff most commonly expressed contempt through emotions and feelings of anger, frustration, resentment, blame, and repulsion.

'Professional and private 'face' activity' ${ }^{31}$ refers to how staff concealed, modified and regulated their feelings during patient interactions. The private or comtemptous 'face' that was presented occurred primarily, but not exclusively in the staff designated zones of the unit, and was a display of feelings, emotions, and behaviours in relation to fatness that exposed their real feelings. In contrast, the professional or caring 'face' was a display of feelings, emotions, and behaviours 'performed' to comply with the healthcare professionals' expected presentations of nursing and medical behaviours. These professional presentations were used to conceal what staff were really thinking about fat patients during interactions. The professional 'face' was most visible during bedside patient interactions.

An important activity of managing 'face' was the conscious regulation of 'the language of fat' that was influenced by the societal subtexts of prejudice. Staff used different language to describe fatness depending on their location within the unit and if the words had an association with prejudicial inferences. The proximity of staff to

\footnotetext{
${ }^{31}$ This concept of professional and private face activity draws on the work of Goffman's (1967) face-work, and Hochschild's (1983) emotional labour (see Section 3.4 and 3.5, respectively).
} 
the patient influenced the language and types of conversations that staff engaged in with the patient and each other. Certain words and phrases were deemed appropriate for different areas of the unit and who was involved in the conversation. At the bedside cryptic communication and euphemisms were used as a polite way of identifying and discussing fat patients. Whereas, in the staff room, fatness was spoken about in derogative ways where the patient's physical appearance was freely and explicitly described and joked about.

'Social awkwardness' refers to the intense discomfort that appeared to be experienced by staff when engaging with fat patients and fat staff in the context of healthcare. There was uncertainty and uneasiness about how fatness could be spoken about and acknowledged in the presence of patients and other fat staff. To alleviate these feelings of discomfort and prevent any unnecessary embarrassment and distress for the patient, staff avoided disclosing information related to the patient's fatness to each other during bedside handovers. When it was necessary to discuss the patient's fatness in their presence euphemisms, secret codes and gestures were used. However, this change in behaviour only served to accentuate the social awkwardness that was present.

'Mutual pretense ${ }^{, 32}$ was conceptualised as an awareness of the fact that everyone knew the patient was fat, including the patient, but direct communication about their fatness was avoided. Consequently, everyone acted to maintain the illusion that the patient was not fat and that they fitted comfortably within the space of the ICU. This mutual pretense meant that staff could avoid conversations about fatness, which might expose staff prejudice or the patients' own embarrassment and insecurities about their bodies. During this mutual pretense staff pretended not to notice the patient was fat, that they didn't fit into the equipment, or were difficult to move around in the bed or into a chair. If the patient did mention their weight or size, staff responded by pretending not to hear the comment, minimalised their weight issue by suggesting they weren't that big, or re-directed conversations to other safer topic.

\footnotetext{
32 This concept of mutual pretense draws on the work of Glaser and Strauss (1965) where it was first described in the care of terminally ill patients.
} 


\subsection{Physical misfits}

Fat patients did not physically fit into the space of ICU despite meeting weight limitations. This had a significant impact on patient care and presented unique challenges for ICU staff. Not physically fitting was related to and determined by the individual's physical body shape, size, weight and reasons for admission. This was illustrated in the care of Agnes who weighed 137kg (BMI $50.3 \mathrm{~kg} / \mathrm{m}^{2}$ ) which was significantly within the normal weight limits of standard care ${ }^{33}$ provision in ICU. Yet the shape of her $137 \mathrm{~kg}$ body (often referred to as peripheral or 'pear shaped" ${ }^{34}$ ) did not fit the ICU environment. When lying in bed concealed by the bed sheets, Agnes did not appear particularly large. However, under the bed sheets Agnes' limbs were extremely large and oedematous, and a large soft apron of stomach rested along her thighs. Her excess skin and fat layers created folds and crevices across her abdomen and upper thighs. When she was moved, this excess skin and fat layers pulled her over and downwards squashing her into places or against safety rails. Parts of her body bulged through any available gap in the physical environment that attempted to contain her. This disproportionate distribution of body fat to her lower extremities meant that folds of skin overhung the chairs, commodes and hoist slings which caused discomfort, sometimes pain, and marking of her skin. Despite Agnes' weight being within standard care limits, her fat distribution posed many care issues, in particular how she didn't always fit into the spaces or devices designed for patient care:

Often with the positioning when you got her on her side she'd end up on the bedrails and stuff and [I] just had to be extra careful with pillows and stuff to avoid pressure areas. I struggled getting the blood pressure cuff to blow up, it would just pop off because it didn't fit particularly well. I couldn't attempt to get her in the shower. I assumed that it wouldn't be very safe because it [shower trolley] doesn't have good sides and she is quite wide... you'd be a bit worried that she'd topple right over (Maggie-nurse, interview, p.4-5).

This narrative demonstrated several factors that impinged on the care delivery for Agnes, in particular problems when she was repositioned in bed, an inability to

${ }^{33}$ The weight limit of standard nursing care in ICU at the time of the study was $150 \mathrm{~kg}$. Beyond this weight specialised equipment, such as the commode and shower trolley, had to be resourced from a central equipment store or hired from an equipment company.

${ }^{34}$ Pear-shaped or peripheral obesity refers to body fat distribution, which is predominantly centralised around the thighs and buttocks (Ashwell \& Gibson, 2009). 
record her health status relevant to blood pressure, and concerns regarding the structure of the shower trolley. The risk to the patient, staff and equipment will be discussed in more detail later in this chapter.

'Correct' positioning and the nurses' ability to move patients in the bed was affected by "the width of the bed versus the patient" (Max-nurse, interview, p.6). Often the beds were "too small in width to be able to safely move someone to the side and move them efficiently" (Vicki-nurse, interview, p.1). Patients, such as Gary, who was as wide as the bed, had arms and stomach folds that over-hung the mattresses, leaving no space to be turned onto their sides. Staff improvised in different ways to accommodate fat patients using every day commonly found hospital items. For example, to create more bed space that could support the overhanging body "bedside tables at the height of the bed with pillows on were used as a ledge on the side of the bed" (Rose-nurse, interview, pp. 2-3). This option of using tables as ledges was preferred over using the wider 'bariatric beds ${ }^{35}$, which posed problems and care management difficulties: "There was a bed [bariatric design] that was wider to nurse these sorts of patients on...//...it used to be really hard to actually reach over the patient in the bed to actually move them" (Ella-nurse, interview, p.5 \& 8).

The ergonomic bends of the bed, that aided patients to sit in an upright position, were also problematic: "You can't position them in the bed because if you sit them up their stomach presses up on their lungs" (Yvonne-nurse, interview, p.5) and "they find it quite uncomfortable to breathe" (Rita-nurse, interview, p.3). When sitting upright in bed breathing appeared more deliberate and laboured as if the person was forcing the air into their chests. Positioning patients in a more reclined position appeared to relieve discomfort and lessened the strained sounds of breathing.

This straining to breathe was most noticeable with Chris. His body had a solid appearance with a large, firm, round stomach that sat like a giant football on his

\footnotetext{
${ }^{35}$ Bariatric beds are specialised beds that are wider to accommodate the physically larger patient and have a higher weight limit of $500 \mathrm{~kg}$ compared to the standard ICU bed weight limit of $267 \mathrm{~kg}$.
} 
oedematous cellulitic ${ }^{36}$ legs. His lower legs were bandaged to soak up the oozing fluid that seeped from his infected legs. The pressure of his stomach left an imprint on his fluid filled thighs. When lying flat on his back the weight of his stomach, which was splayed out by the effects of gravity, pushed his abdominal content into his ribcage. Each breath in was a struggled attempt to force his displaced stomach back out of his ribcage. As the muscles relaxed during exhalation his stomach jolted back down again rapidly expelling the air from his lungs. Chris appeared tired and distressed by the efforts of breathing against the weight of his stomach and he became fidgety and restless the longer he lay on his back. When Chris was sat upright in bed, his stomach became scrunched up and squashed upwards under his ribcage. His chest appeared to lift upwards and over the bulge of his stomach that pressed into his ribcage creating a heaving pattern of breathing. This type of experience was observed in other fat patients when breathing was obstructed by their large stomachs. To reduce the pressure on patients' stomachs and lungs they were often only sat up to a 45 degree angle while in bed or if possible the whole angle of the bed tilted downwards to release some of the pressure of the abdomen from the chest. This tilting motion of the bed appeared to provide some relief to Chris' laboured breathing as the heaving pattern of breathing became less noticeable (Chris-patient, field notes, p.3).

Patient positioning in the bed was hindered by the frequent failure of the motorised components of the bed. The beds were not able to support the unequal weight distribution of the patient. This was seen in the case of Rawiri whose fat was centrally distributed around his abdomen. He had a firm solid stomach that protruded out in front of him and rested on his upper thighs. His arms were relatively slim and he had thin bony legs deplete of any noticeable muscle mass from being wheelchair bound for more than 20 years. On many occasions the motorised bed failed to lift the backrest up to position Rawiri into a seated position. This failure appeared to be caused by the bulk of his $132 \mathrm{kgs}$ resting on the motorised section of the bed as opposed to being equally distributed over the full bed surface. When this failure occurred the nurses manually pulled up the backrest to the point that the motorised section of the bed could take his weight. Usually this was once the backrest was raised

\footnotetext{
${ }^{36}$ Cellulitis is an infection of the skin caused by bacteria leading to red swollen painful areas of skin (Gunderson, 2011).
} 
above 30 degrees. The nurses were accustomed to dealing with this bed failure and became a part of normal practice. Rawiri appeared to be unaware that his weight was the cause of the failure or that the failure of the bed mechanics had occurred at all as he continued to chat away with the nurses (Rawiri-patient, field notes, p.4).

The 'type' of fat body and how the patient occupied the bed determined the ease to which the patient could be correctly positioned and moved in bed. Soft, flabby, fat bodies with many rolls of sagging skin that filled the width of the bed posed particular challenges: They were "difficult to balance on their side...difficult to stop the momentum once you start moving them" (Rebecca-nurse, interview, p.5) and "you don't actually get them off the[ir] pressure area[s]" (Phillippe-nurse, interview, p. 6). These examples highlight how the pendulous, loose, overflowing nature of this type of fat body impinged on the person's normal centre of gravity when lying in bed on their side and their ability to relieve pressure contact with the bed.

Pressure care challenges were observed in the care of Agnes as "whichever position she was in there was another pressure area, like under her breasts or under a [skin] fold and in her bottom" (Sue-nurse, interview, p.6). She had a small persistent reddened area on her buttock, an ulcerated area on her left ankle and excoriated skin in the folds of her breasts and thighs. When turning her in bed, nurses pulled randomly at her excessive loose fat rolls not knowing "if it was a skin fold or breast they were holding" (Robyn-nurse, interview, p.1) in an attempt to secure a firmer grip on her body to initiate movement. This 'looseness' of Agnes' weight gave her an "extra jolt at the end of turning" (Rose-nurse, interview, p.3) where the momentum of her body propelled her to the extent that she was at risk of rolling off the other side of the bed. It required the presence of extra nurses on both sides of the bed to prevent this from happening.

In contrast, firmer, bulky, solid, more muscular appearing fat bodies were less challenging to position and move about the bed: "quite tight bodies you can turn them quite easily ... you can get a better turn because you're actually moving their whole body (Phillippe-nurse, interview, p. 5-6). This difference in fat distribution was observed by staff in the cases of Rawiri and Agnes: "He was solid so when you 
moved him it was easy to roll him whereas Agnes was sort of flaccid" (Stella-nurse, interview, p.4). The firmness of Rawiri's body and his solid shape meant that he occupied less width of the bed, had less skin contact with the bed and seemed to fit better than Agnes.

Additionally, the height of the fat person contributed to the ease to which the nurses could care for the fat patient in bed: "It's much easier to turn and position a taller obese patient than a shorter one" (Sandy-nurse, interview, p.4). This was because taller fat patients had "more length to get access to" as opposed to nurses being "shoulder to shoulder packed in [to] access their body" (Sandy-nurse, interview, p.4). Rawiri's tall stature allowed for ample space for the three nurses, positioned on the same side, to turn Rawiri and not be impeded by their colleagues presence. In contrast, Agnes' short stature had meant that three nurses were squashed up together as they positioned themselves to take hold of the Agnes' shoulders, waist, and hips to initiate the turn.

As with fitting into beds, there were difficulties regarding 'fit' with other equipment used in the ICU to monitor, record and support patient care. The selection and availability of the correctly sized cuff $^{37}$ used to record non-invasive blood pressure was problematic. They were either too small "to go around [the patients arm]" (Phillippe-nurse, interview, p.3), or the velcro was observed to rip apart as the overlap on the cuff was not enough to withstand the inflation pressures. In both instances the capability to record blood pressure was impaired by the inability to secure the monitoring device. In these circumstances nurses used various techniques to make the 'large adult' sized cuff fit securely around the patient's arm. To secure Gary's cuff nurses would wrap their hands tightly around the cuff during inflation to prevent the velcro from pulling apart or attempt to wrap the cuff as tightly around the arm as physically possible before initiating cuff inflation.

${ }^{37}$ Blood pressure cuff size refers to the inflatable bladder component. The correct size covers $80 \%$ of the circumference of the mid upper arm and $2 / 3^{\text {rd }}$ of the distance between the shoulder and elbow (Pickering et al., 2005). 
The 'thigh sized ${ }^{38}$, cuff recommended for fat patients whose arms are too large for the 'large adult' cuff (Bariatric transport policy, material artefact, p. 3), was never observed to be used in practice, instead nurses struggled to make the 'large adult' cuff fit the patients' arms. One reason for not using the thigh cuff was that the name of the cuff acted as a deterrent: "I wouldn't consider using the thigh cuff on the arm it doesn't seem right to use something on the arm that's meant for the thigh" (Phillippe, interview, p. 13). When the cuff did successfully inflate concerns were raised about the accuracy of results when inappropriately sized cuffs and techniques for securing the cuff were used: "then to trust whether that blood pressure was measuring right" (Phillippe-nurse, interview, p.3). The implication was that such ill-fitting monitoring devices may have produced false records ${ }^{39}$ of a person's blood pressure potentially changing the course of action taken by the nurse.

In addition to the problems of securing the blood pressure cuff around the arm of a fat patient, the cuff left very clear observable skin markings. Often a fat person's arm appeared streaked with vertical white coloured pinch marks and bruising where the cuff had squeezed tightly around the excess fat and skin folds. These marks remained for the duration of the admission and were compounded by the hourly repetition of the procedure. The markings were most noticeably seen on Agnes, Emiri, and Gary due to their large amounts of arm fat and excess skin. This simple monitoring process also caused discomfort. Emiri appeared particularly troubled by the discomfort caused by the cuff. She screwed up her face and looked towards her partner for recognition of the pain and acknowledgement of the bruising the cuff was causing to her arm. Nurses were also affected by the procedure demonstrated in their reactions to the marks that they left. On removing the cuff they would occasionally rub the patients' marked arm while apologising for the damage and discomfort caused (Emiri-patient, field notes, p.20).

Further issues of discomfort arose from the structure and design of the standard and bariatric equipment which didn't always accommodate the different fat

\footnotetext{
38 'Thigh sized' cuff is the name assigned the cuff originally designed for use on adult thighs and is the next sized cuff after 'large adult'. The increase in the arm circumference of fat patients means that their measurements roughly fit thigh cuff parameters (Pickering et al., 2005).

${ }^{39}$ Too small a blood pressure cuff produces falsely high readings (Araghi, Bander, \& Guzman, 2006; Pickering et al., 2005).
} 
body shapes: “They don't necessarily find our bed or our chairs particularly comfortable" (Glenda-nurse, interview, p.2). Discomfort appeared differently for patients depending on their body fat distribution: "If people are quite pear shaped with very soft excess skin it's quite difficult to get them comfortable in the [hoist] slings. Make sure that there's no pinching. To get them in a comfortable position" (Ritanurse, interview, p.2-3).

Different body shapes affected the comfort levels experienced by patients when using specific equipment such as the bed, hoist and chairs. Agnes had preferred to stay in bed each day as the process of hoisting her into the bariatric chair had been painful and the chair itself uncomfortable. As the hoist sling took the full weight of Agnes overhanging body she grimaced and called out in pain. Her large oedematous thighs and bottom bulged out around the relatively thin thigh straps which were digging into and pinching her skin folds. The excess skin and fat of her lower extremities was pushed through the large gap in the bottom of the sling by the weight of her body bearing down on the sling straps. On one occasion, Agnes' catheter became trapped between her skin folds and the sling which ruptured a section of the catheter that secures it into her bladder causing the catheter to fall out on the floor. A persistent reddened imprint of the sling was left on her thighs and buttocks from the short duration (less than two minutes) that the sling had fully supported Agnes body weight as she was moved from the bed to the bariatric chair (Agnes-patient, field notes, p.11).

The bariatric chair, used for Agnes, was specifically designed to accommodate patients up to $200 \mathrm{kgs}$. It had motorised back and leg rests to assist with patient positioning and could be reclined almost to a bed position. The seat dimensions were wider and deeper than standard hospital recliner chairs and the arm rests higher to support the larger patient's body. However, due to Agnes' body shape her bottom and legs didn't fit into the seat. Her stiff oedematous legs were too short for the depth of the seat and her thighs and rolls of stomach overhung the armrests. To overcome these problems the leg rest was raised to support her legs, and pillows were placed under her bottom to raise the height of the seat level with that of the arm rests creating a 
wider seat. This modification was somewhat successful in relieving the discomfort of having arm rests digging into her thighs and used as a temporary measure.

Squeezing patients into equipment when they were clearly not going to fit comfortably was often the only option staff had in conducting cares. This practice of 'forcing' patients to fit occurred regularly when patients had no choice but to use illfitting equipment: "I know what you're thinking, she won't fit. Ah watch this" and Hilary lifted up the arm rests to the commode to create a little bit more space and proceeded to squeeze Emiri into the commode seat (Emiri-patient, field notes, p.1). It seemed that Emiri did just about fit on the commode seat although, her bottom and thighs were bulging out over the sides and through the toileting hole and almost touching the plastic collection pan underneath. When Emiri stood up the commode seat was momentarily stuck to her from the wedged in buttock skin and a reddened imprint of the back of the commode and semi-raised arm rests were left on her thighs, hips and sides of her body. Every time Emiri used the commode she complained about how uncomfortable it was to use and grimaced when attempting to extract herself from the seat when done.

Even in death, the fat body did not fit: "It [body] just barely fit in the mortuary trolley" (Maggie-nurse, interview, p.12). The mortuary trolley was often too narrow: "To fit her in we had [to] just kind of hold her sides and push things in a little bit" (Caroline-nurse, interview, p.2), or not deep enough to accommodate patients with large stomachs: "The lid on the morgue trolley didn't fit on properly, it was just hitting the top of her belly" (Caroline-nurse, interview, p.2). When the patient was removed from the trolley for viewing by the family the body was left visibly marked by the pressure of being squeezed in too tightly: "Her abdomen and her thighs [were] mark[ed] from where she's wider than the trolley" (Caroline-nurse, interview, p.3). Once in the viewing room, the table was too small which meant the deceased body overhung the sides: "Limbs [were] hanging down the sides and things, it was really hard to keep her on the trolley [table] (Maggie-nurse, interview, p.12).

In these instances staff discussed their embarrassment in relation to how undignified and disrespectful it appeared to family members to be squeezing and 
pushing the body into a space that was clearly too small: "The family were there...watching and she clearly doesn't fit properly. I found that quite embarrassing" (Caroline-nurse, interview, p.2). Likewise, embarrassment was voiced by staff when discussing how they felt about transporting the deceased body to the mortuary: "I felt quite embarrassed because we were about to go through all the corridors of the hospital to get to the morgue" (Caroline-nurse, interview, p.2).

Not only did staff describe how fat patients failed to fit into ICU but they also discussed how they didn't fit into other clinical environments such as radiology departments and during inter-hospital transfers via aeroplane and ambulance. Again, in these situations it was often not the actual weight of the patient but their physical shape and size that caused them to not fit: "It wasn't a weight limitation. It was a physical [one] ....physically we couldn't gather him together enough to fit him inside the scanner" (John-doctor, interview, p.1). Similarly, fat patients couldn't be squeezed into the ICU transport stretcher which had an equipment bridge that swung out over the patient's central abdominal area:

Where the bridge comes over, the ventilator and the monitor was pushing into the top of her thigh and pushing her stomach...I was having to prop her physically onto the stretcher with my knees because I was worried she was going to fall off (Shirley-nurse, interview, p.2).

In this example the implications of not physically fitting created risk as the patient was at risk of injuring themselves on the equipment or if they fell off.

\subsubsection{Consequences of being a physical misfit}

The risk of phyiscal injury was a significant consequence of not physically fitting for both the patient and staff and evoked much anxiety. Nurses were specifically concerned and voiced feelings of fear with how the fat body 'not fitting' could get physically injured or cause injury to staff trying to assist with care: "It's the fear of them falling" (Mary Anne-nurse, interview, p.2),... "I get worried I'm going to drop them" (Sophie-nurse, interview, p.1), and..."there's that fear of being pushed over by them" (Roma-nurse, interview, p.1). This increased risk of harm was from falling or being dragged out of bed by the momentum of their large pendulous 
stomachs on turning, or by being dropped by the staff unable to support their unbalanced weight distribution as they stood on their unsteady feet. These concerns of physical injury featured prominently when nurses discussed using equipment that aided patient transfer: "I get worried that they're going to fall out of the sling or the sling's going to break" (Sophie-nurse, interview, p.1). During Agnes' stay in hospital the ward hoist she had been in broke midway through transferring her from the bed to the chair: "Fortunately she was hovering over the bed, so she just fell back on the bed" (Maggie-nurse, interview, p. 4).

Likewise, turning patients in bed evoked anxiety about the risk of physical injury: 'sometimes people are so large that their tummy will actually drag them out of the bed and push the nurses away that are pulling them over" (Roma-nurse, interview, p.3). To prevent patients from rolling out onto the floor bed rails were often left up during the turn particularly if the patient filled the width of the bed or had a large stomach. This practice was regularly observed in the care of Agnes, Emiri and Gary.

Nurses had the added burden of managing a damaged body or protecting the body from further damage. Fat bodies usually (over and above the actual medical reason for the admission) came to the ICU physically damaged and the nurses had to manage that damage whilst at the same time protecting the body against further damage occurring: "Often people will come in with pre-existing skin damage underneath folds and you're trying to look after that and heal anything that's already existing or prevent problems" (Caroline-nurse, interview, p.4). Preventing further damage was a concern for nurses when caring for Gary. When turned in bed care was taken to ensure Gary's 'open abdomen' ${ }^{40}$ was never squeezed up against the bed rails or any unnecessary pressure placed on his wound site. This often meant that pressure areas were never fully relieved despite the use of a pressure relieving mattress. Gary's fear of moving meant that he was reluctant to change positions putting him at "high risk of pressure ulcers" (Roma-nurse, interview, p.2). Despite this risk he preferred to

\footnotetext{
40 'Open abdomen' is a complication of abdominal surgery where the closure of the abdomen would cause significant pressure within the abdominal cavity leading to abdominal compartment syndrome and possible multi-organ failure (Regner, Kobayashi, \& Coimbra, 2012). Therefore, the abdominal muscles and skin are not fully sutured together leaving the abdominal content visible under the transparent dressing.
} 
continue to remain squeezed into a chair that was obviously causing discomfort and pain.

This fear and appreciation of the clinical risk the 'open abdomen' posed was reflected in Gary's interactions with staff. Gary liked to make jokes, tease the nurses and retell stories to entertain staff however, when the conversation about changing positions in the bed or getting out into the chair occurred his demeanour changed. His behaviour indicated that he was fearful and anxious about the additional risk posed by his fat 'open abdomen'. He became serious and short-tempered with nurses who continued on with the light-hearted conversations. Often nurses didn't recognise his behaviour change as being related to his fear and anxiety and the more his anxiety went unrecognised the angrier and discontented Gary became with his nursing care. To manage his fears and anxiety whenever possible, Gary refused to be moved or negotiated with staff a slight position change in bed instead of getting out into the chair. Similarly, Gary would ask for assistance instead of bending over his stomach: "I can't reach my legs to wash them, my stomach will fall out" (Gary-patient, field notes, p.10).

Most noticeably, Gary's demeanour changed when staff were organising the equipment and other nurses to assist with mobilisation into the chair. During this time, Gary frequently asked if the nurses knew what they were doing and when Gary was assisted to a standing position he held his stomach, closed his eyes and would ask: "Is my stomach falling out?" (Gary-patient, field notes, p.2). On one occasion Jade, a nurse, reassured Gary that she had "seen many 'open abdomens' and only once seen one 'fall out' so the chances of it happening were very slim" (Gary-patient, field note, p.10). Once sat in the chair Gary would always ask the nurse to check his wound site. On confirmation that his abdomen was still intact, he immediately began laughing and joking again with the staff and the rigidness of his body relaxed back into the chair. This erratic behaviour of being jovial then angry and questioning of the nurses' abilities, to being relaxed and jovial again irritated staff. Towards the end of Gary's admission, during conversations between staff in the staff room, Gary became known as the "grumpy miserable guy... who makes it difficult to be cared for" (Gary-patient, field notes, p.24). 
The worry of physical injury extended beyond the patient to the nurse as many of the nursing cares such as washing, repositioning, and mobilising involved the whole body which increased their personal risk of injury: "you know there's always that high risk of hurting yourself if someone's particularly big" (George-nurse, interview, p.2). In particular, this concern was in relation to sustaining a muscular injury to the neck, shoulders or back: "Well the solid heavy ones normally I walk away with a sore back" (Yvonne-nurse, interview, p.1)..."doing a dressing that's really bad for your back because the angle that you have to be at for a prolonged period of time" (Joanne-nurse, interview, p.18). These fears about injury were real for the nurses as recently one nurse had sustained a minor back injury from redressing a perineal ${ }^{41}$ wound which had required the leg to be elevated for up to 30 minutes at a time. As a means to prevent similar injuries the incident had resulted in the purchasing of disposable leg hoist slings.

Risk taking and accepting the increased risk of harm occurring appeared to be a recognised consequence of not physically fitting: "There was a consultant anaesthetist and myself who went down there [radiology] and physically tried to fit him in because we believed that the benefits of the scan outweighed the risks" (Johndoctor, interview, pp.1-2),..."There's going to be significant risks with the transfer" (James-doctor, interview, p.10). Equally, fat was considered to be an additional risk factor to the safety and wellbeing of the patient when judgements were made to accept the potential harm that could occur as staff improvised to accommodate the fat body. One example of accepting this risk was during aeromedical transfers of fat patients who exceeded 'safe carrying capacities' of equipment. In these instances decisions were made to transfer these patients on 'mattresses on the floor' and without adequate safety restraints. Patients were often uncomfortable and in pain during the transfers: "they're often spilling out over the stretcher so their limbs are touching the frame of the aircraft which means they get a lot of vibration which increases their pain" (Rebecca-nurse, interview, p.11).

\footnotetext{
${ }^{41}$ Perineum refers to the anus and genital areas of the body.
} 
Nurses were observed to take risks when pushing fat patients in their chairs from one bed space to another or when taking them off the unit as had occurred with Emiri and Agnes. Emiri needed to be moved from her bed space to the bariatric room for renal dialysis. She was too weak to walk the short 30 metre distance to the room so had to be pushed in her bedside chair. It required two nurses and two family members to push, pull and steer the chair around the unit. Navigating the chair around the bends of the unit and into the correct place in the new bed space appeared cumbersome and difficult and resulted in many collisions with equipment and other patient beds. The nurses had to push hard to start the chair moving by pushing their back or side of their body against the back of the chair. Changing direction required a nurse to physically block the way and twist the chair to face the new direction of travel. In doing so, the nurses were awkwardly twisting their bodies to initiate movement, realign the chair, or stop the chair's momentum (Emiri-patient, field notes, p.16).

Added to this worry of physical injury was the concern of not having professional indemnity: "You need to ensure that you don't exceed what you're supposed to lift because you may not be covered if you do something stupid" (Bobnurse, interview, p.2-3). This meant that it was not uncommon for the quality of patient care to be compromised in order to protect the nurse from harm:

The most important thing you have to do is protect yourself first and keep the patient safe... if it means a patient doesn't get turned, I know that isn't acceptable in some ways but if you hurt your back and end your career, then that's certainly not acceptable (George-nurse, interview, p.6).

Nurses often reported feelings of conflict between doing the best for their patients and protecting themselves from harm: "It's just not even worth trying to do things on your own you'll just hurt yourself. But then I get frustrated that I'm not giving good care to people if I can't turn them regularly" (Ella-nurse, interview, p.3). This increased personal risk in combination with "harder nursing work compared to patients that aren't obese" (Caroline-nurse, interview, p.1) meant fat patients were a less desirable choice during shift allocation: "I wouldn't choose an obese patient to look after just from my point of view of protecting myself, my career" (Roma-nurse, interview, p.4), and created some reluctance to assist others: "getting people [staff] to 
help can be a challenge because if people have had previous back injuries and back problems they won't be very keen" (Shirley-nurse, interview, p.2).

\subsection{Medical misfits}

Fat patients had distorted bodies with altered physiology. This meant that they did not fit into medical norms of understanding the human body and accepted ways of practising medicine. This created technical difficulties for doctors in practice which ranged from being "unable to place a chest drain because fingers were not long enough $^{42}$ to actually make it through to the pleural space" (Alexis-doctor, interview, p.3), to not being able to actually "get central lines, arterial lines, [and] IV access into obese patients" (John-doctor, interview, p.1) because the excess fat concealed the normal anatomical positioning of arteries and veins. Often this difficulty meant that it could take "an hour or an hour and a half just to put a drip [intravenous line] in" (David-doctor, interview, p.2) compared to less than ten minutes in a normal weight patient.

Nurses discussed similar clinical concerns as doctors in regards to anatomical, physiological and technical issues, such as during the insertion of a urinary catheter: "Catheterisation was quite difficult...Trying to get to her urethra with lots of rolls around her legs. I needed extra staff to help support the legs apart and support the vagina" (Kate-nurse, interview, pp.1-2), or during a cardiac arrest: "I wouldn't be able to compress enough on their chest wall to make the compressions effective" (Sophienurse, interview, p.2). These clinical risks increased the likelihood of an adverse event occurring or of a longer hospital stay: “They're probably going to have more complications in the ICU so those complications might delay their recovery" (Jamesdoctor, interview, p.5).

\subsubsection{Consequences of being a medical misfit}

The presence of excess fat increased the risk of harm occurring to the patient and was a significant consequence of not medically fitting. In particular, medical risk

\footnotetext{
${ }^{42}$ Doctors use their fingers to create a path between the ribs into the pleural space for the chest drain to be inserted (Angaramo, 2010). The excess chest fat means that the doctors' fingers are often too short to reach the pleural space where the tip of the chest drain must be positioned.
} 
occurred when the distribution of excess fat tissue hindered medical interventions and was a concern that underpinned clinical decisions: "[I'd] be more inclined to talk to my boss early because an overweight person is probably more likely to decompensate ${ }^{43}$ quickly" (James-doctor, interview, p.5), and assessment and management strategies: "Listening to the chest or feeling the tummy you're probably a lot more likely to miss things just because of the large fat layer" (James-doctor, interview, p.4).

Doctors were specifically concerned with how the presence of fat altered the physiology of the body, increased the risk of complications, and impacted on the highly technically invasive procedures that are often the mainstay of ICU treatments: “There's a fear, physiologically there's a fear. Anaesthetists are scared of big people because of their airways and their [lung] capacity and I'm concerned with big people because of the technical aspects" (David-doctor, interview, p.2). In this example, risk for patients arose from both physiological differences and technical challenges. Technical difficulties appeared to evoke the most fear for ICU doctors as this directly related to their own clinical skills and abilities. The emphasis on risk in these situations was about being unable to master the increased technicality that fatness imposed on effectively and efficiently conducting procedures.

To minimise the risk that the fat body posed, doctors often opted to proactively insert lines that may be needed in an emergency. This anticipatory management extended to intubating patients, a procedure not without its own risks, as a precautionary measure: "Things could go bad quickly on a plane we had to intubate them and sedate them for the journey just because they were obese" (James-doctor, interview, p.3). This pre-emptive culture of risk management illustrated how fatness was seen "as a complication of management" (David-doctor, interview, p.1) of the patient's primary reason for admission: "It [fat] does hinder the ability to investigate and manage a patient" (John-doctor, interview, p.1).

${ }^{43}$ Fat patients have low/poor physiological reserves to accommodate increasing physiological needs. Therefore, their condition/ health can rapidly deteriorate because they are unable to compensate for these changes that are occurring at a cellular level (Bahammam \& Al-Jawder, 2012). The term 'decompensate' in this context refers to the physiological inability to cope with the current disease/ illness leading to significant deterioration of the patient's condition. 
'Fat' complicating the medical management of care was most noticeably seen in the care of Gary. On admission to hospital, it had proved challenging to make a definitive diagnosis of Gary's presenting symptoms. Undertaking a physical examination of his painful abdomen was difficult and the clarity of the abdominal investigations was impaired by the excessive fat surrounding his abdominal organs. His management was particularly complicated by him not being able to 'fit back together again' following abdominal surgery despite having 10 kilograms of fat removed from his abdominal wall. During surgery his abdominal content had become extremely swollen from the effects of the hernia and being physically manipulated in theatre. As a result, Gary was admitted to the ICU with an 'open abdomen' where the skin and muscle were only partially closed around his swollen abdominal organs.

The fat 'open abdomen' posed a significant clinical risk for respiratory and abdominal complications. To optimise lung function and prevent a chest infection it was important that Gary sat upright in a chair. However, this upright position increased the pressure on the sutures holding Gary's wound loosely together. This pressure increased the risk of the sutures ripping through the stretched skin leaving the abdominal content exposed. This complication and risk was the focus of every ward round discussion where doctors debated how best to manage the abdomen in its current state and what would be the safest and most appropriate management plan for closing the abdomen.

\subsection{Social misfits}

Outside of the clinical context, staff spoke about how fat bodies didn't fit within wider physical and social spaces such as on buses and trains. These experiences influenced their responses and attitudes to fat patients in ICU. Staff provided many stories of experiences where their own personal and social spaces had been disrupted by the physical presence of a fat person:

I sat next to someone who was obese and I got a tiny amount of seat and they took up the whole seat. I had a terribly uncomfortable ride...because I was squished in next to them. I sort of sat on one side pretty much pushed against the glass for two hours (Vicki-nurse, interview, p. 10). 
The dominating physical presence of the fat body within social spaces affected how staff socially perceived the fat patient within the ICU. Fat patients were often discussed in ways that revealed social difference, disapproval and judgement:

We see them as not helping themselves. They're generally not working because they can't because they're obese. They're not eating healthy they're not exercising like we all who aren't obese. And not generally seen as maybe giving to society as much as what we do. The people that you see who are obese are sitting round eating. We're eating healthy and we're exercising and making the effort... we judge them on the fact that they're not (Jenny-nurse, interview, pp. 7-8).

This narrative typified many of the views expressed by ICU staff that fat patients were seen as social misfits, by demonstrating they were socially different to normal weight individuals through their judgements and disapproval about the ways that they chose to live.

Staff spoke in ways that grouped fat people as being "different to us" (Phillippe-nurse, interview, p. 8) through dialogues that referred to 'them' the fat patient/person and 'us' the normal weight person: "They have a problem with putting food in their mouths that they can't stop...[I've] always been fit and eaten well" (Vicki-nurse, interview, p.8-9). Comparisons of lifestyle practices were used frequently to assist in amplifying the differences between the groups: "[They're] just not taking very good care of themselves. I make a fairly good effort to eat healthy and have regular exercise" (Maggie-nurse, interview, p.11). Fat people were viewed as lazy, unmotivated, and having no willpower and self-control to make the right choices to be healthy: "I think of overweight people as being a bit lazy and not [having] particularly good self-control" (Maggie-nurse, interview, p.11), and were considered to be "not quite as good as you are" (Phillippe-nurse, interview, p. 8). Likewise, the fat physical body was socially differentiated as being imperfect and grotesque: "It's not attractive. Seeing rolls of flesh it's not appealing, it looks messy. It doesn't look right" (Sandy-nurse, interview, p.11).

Fat patients were further segregated through the use of language that objectified them: "Oh you can't put 'that' in an ambulance or you can't put that 'load' 
in an aircraft" (David-doctor, interview, p.11), .. "We are thinking about who [which nurse] would be okay taking 'it'...I wouldn't want to be giving 'it' to someone that's had a recent back injury" (Florence-nurse, interview, p.5), ... "They were calling her it" (Trudy-nurse, interview, p.9),... and "it's so bloody fat" (George- nurse, interview, p.10).

The patients themselves were acutely aware of their differences: "They're embarrassed and they know that they're a challenge to move and turn" (Rose-nurse, interview, p.2). Patients frequently apologised about their size and for the difficulties that their body posed to staff. Gary had been particularly embarrassed and apologetic to the nurses for his appearance when he required assistance to wash his lower body, legs and feet which he found difficult to reach due to his large protruding stomach. His fungal infected toe nails were overgrown and his skin around his heels was dry, cracked and ingrained with dirt. When he had his feet washed to remove the dirt and foot odour he repeatedly said "I'm sorry it must be horrible for you? I'm sorry..." (Gary-patient, field notes, p.11). Similarly, Emiri kept apologising every time she needed assistance with mobilising or getting into a more comfortable position. She often started any request for assistance by saying "sorry ladies I don't mean to be a nuisance again but..." (Emiri-patient, field notes, p17).

Fat patients were considered to be different but 'the same' as other social groups with stigmatising diseases such as alcoholism and nicotine addiction where socially constructed deviant behaviour was to blame: "It's self-inflicted, a bit like alcoholism or a bit like somebody who doesn't take their asthma inhaler" (Daviddoctor, interview, p.2). Blaming patients "because it's of their own doing" (Vickinurse, interview, p. 10) for not taking responsibility for their own health formed a large component of the discourse in morally judging fat patients.

\subsubsection{Judging fatness}

Fat patients were judged both morally and clinically as a consequences of being misfits within the ICU. Moral judgements were based on belief that fatness is the result of personal failings: "they had the choice but they didn't make the choice to control their weight" (Joanne-nurse, interview, p.10). Clinical judgements were often pragmatic in nature and focused around the physical size of the fat body: "Will they 
fit the bed? Can we get a mattress quick enough to get them admitted?" (Florencenurse, interview, p.14), or identification of fat related co-morbidities: "someone who is obese having difficulty breathing I think they’ve got undiagnosed sleep apnoea" (Alexis-doctor, interview, pp.1-2),...."Got to be a bit more vigilant to look out for diabetes that hasn't been diagnosed" (James-doctor, interview, p.2), and specific management strategies such as those which avoid general anaesthetics: "[The] general surgical team to come back today to close wound by local anaesthetic. Not for OT [operating theatre]" (Gary-patient, medical notes, p. 32). These examples demonstrate how knowledge of the 'medicalised' fat body affected the clinical judgements made. These types of clinical judgements were often infused with moral discourse/dialogue:

If I take a referral from another hospital there's a judgement passed on. Often both doctors will laugh in a very dark way about somebody being obese... We're very happy to say oh you know the 'big unit' and make a sort of judgement on it (David-doctor, interview, p.5).

Moral judgements were often implied during clinical conversations through sarcasm and irony: "they'll be wearing extra oversized clothing I can tell you from here...this man was not size small" (Robyn-nurse, interview, p.7) and "maybe they should pick the salad option at McDonald's next time" (Julian-doctor, interview, p.9). This type of discourse implied that there was an intention to impart a judgement about the fat person's choices in life. Moral judgement of the fat body infiltrated all aspects of the culture of the ICU and was embedded in conversations when clinically discussing fat patients. It was commonplace practice during informal handovers to "hypothesise on how they got like that" (Stella-nurse, interview, p.11): "Does she just sit there and eat KFC all day?" (Stella-nurse, interview, p.11) or to imagine the effects of fatness on the body: "she had to be specially filtered to get rid of this stuff [soluble fat from her blood] and we were all just like what the hell did her blood look like" (Stella-nurse, interview, p.11). Often these types of conversations contained derogative physical descriptions of patients: "she is as wide as she is tall...the size of a boulder" (Julian-doctor, interview, p. 9) and "this person's a tub of lard" (Phillippenurse, interview, p.7), or suggested a flaw in the person's physical ability and health: "made for comfort not for speed" (Kate-nurse, interview, p.11) and "they're a sitter 
for MI [myocardial infarction ${ }^{44}$ ]" (James-doctor, interview, p.11). In each of these examples the implication was that the fat body is distorted and blemished because of the person's behaviours, such as poor diet and sedentary lifestyle, that it was their own fault, and that they were 'personally' responsible for their present condition.

Judging fatness during clinical conversations appeared to influence clinical decisions to admit fat patients:

There are times where you actually look at the patient and go 'oh they are big'... and you just think oh should we actually be doing this [admitting the patient]. You step back a lot more to get a bigger picture just to see what you're actually doing (Julian-doctor, interview, p.2).

Nurses often interpreted medical discussions about the presence of fatness as being the most significant defining factor for some decisions that were made:

You know the fact that they're 180 kilos is somehow influencing the decision about whether to treat or not treat or to admit or not ... in the long list of things [co-morbidities] that you're considering, it's kind of like the elephant in the room in a way that you don't, you would never name as the defining factor (Rebecca-nurse, interview, p.12).

This type of narrative suggested that "medical decisions are actually biased" (Julian-doctor, interview, p.2) and that the co-morbid effects that fatness had on the patient's ability to recover from illness were used as a means to justify admission decisions:

I certainly would never use obesity as a reason for not admitting someone to intensive care. Now having said that there are obviously a lot of illnesses associated with obesity and those may be factors as to whether I shouldn't admit them to ICU but the obesity itself is not the determining factor (Johndoctor, interview, p.3).

This suggested that mentioning fatness in medical conversations changed the medical interpretation of the information presented about the patient, and tainted the decisions and perspectives of the medical team. In particular, the idea that fatness is detrimental to patient recovery thus, influencing decision-making processes:

${ }^{44}$ Heart attack 
If you send me a slim 69 year old man with advanced COPD [chronic obstructive pulmonary disease] and heart failure and some venous leg ulcers and diabetes and high alcohol intake. And then you send me the same patient but at 160 kilos I have a totally different impression (David-doctor, interview, p.10).

When judging fatness, moral viewpoints on fatness were justified using clinical rationale for the effects on the person's health:

What it [fatness] does physiologically to the person's development, to their bone structure, to their nervous system, to their cardiovascular system...they're just waiting to come into hospital to have heart surgery or their hip replaced...something needs to be done. We can't have the ambulance at the bottom of the cliff, that's just going to ruin the health system (Lee-nurse, interview, p.13).

This type of clinical and moral judging was present throughout the data and led to staff expressing feelings of contempt towards fat patients.

\subsubsection{Contempt}

Contempt was frequently demonstrated in the behaviours and expressed emotions and feelings of staff when caring for fat patients: "Because you feel they haven't taken responsibility and it's their fault that they're there...You sound somewhat unsympathetic or harsher. Your sentences and the way you treat them come out differently whether you were conscious of it or not" (Joanne-nurse, interview, p.11). This cool disregard for fat patients occurred as staff criticised and condemned fat patients from a position of assumed supremacy: "You do naturally make a judgement that they're not quite as good as you are, you get a feeling of superiority you'd look at somebody and you'd go I would never get like that" (Phillippe-nurse, interview, p.11).

Staff most commonly expressed contempt through emotions and feelings of anger, frustration, resentment, blame, and repulsion. These feelings were most apparent during discussions about intimate personal cares: "I've had gloves that went up to my armpits to get under skin folds I find that it makes me just feel sick" (Vicki- 
nurse, interview, p.7), the fat body's presence: "The smell was unbearable, she had lice, she had in-ground dirt in places I can't even go...it was disgusting" (Shirleynurse, interview, pp10-11), physically moving patients: "I do have an inner anger towards it [them] because it's my back" (Yvonne-nurse, interview, p.7), patient motivation: "I've got very frustrated because they've been unwilling to help themselves and I've felt myself physically hurting" (Glenda-nurse, interview, p.11), and patient responsibility: "I feel a little bit cross with the patient for getting themselves into that state where I[' $\mathrm{m}$ ] having to look after them" (Ella-nurse, interview, p.11). However, these emotions were often hidden from the fat patient and usually only visible during interactions with other staff within staff designated zones of the unit or when away from the patient's bedside.

\subsection{Professional and private 'face' activity}

The management of professional and private 'face' activity was evident both in the observations of practice and in what staff reported during interviews. Therefore, the observational and interview data have been merged together to create a cohesive description of the ICU culture. The proximity of staff to the patient influenced what 'face' was presented to others and what emotions and feelings were expressed. Staff maintained an acceptable professional demeanour when engaging in bedside patient care through modifying and regulating their emotions and feelings to present a professional 'face': "If you don't like someone you kind of over compensate a bit with a patient, you'll be really, really nice to them" (Stella-nurse, interview, pp.12-13).

At the bedside staff demonstrated empathy and kindness: "I try to be empathetic towards all my patients especially when I see someone trying to move and struggle to get by" (Bob-nurse, interview, p.11). Gary had been criticising his physical appearance while being assisted with a wash. His nurse, Jade, had responded positively with encouraging statements about his weight and his relationship with food. During this conversation she never once reaffirmed what Gary thought about himself. While soaking his feet in the bowl of hot soapy water he had commented that this was the best care...//...that he didn't feel the nurses were discriminating against him (Gary-patient, field notes, p.11 \& 8). Likewise, Emiri had spoken highly of the care she received from staff: "I felt cared for by the staff...Ella somehow humanised 
the care she gave...Elaine was very caring, she touched my hand or stroked my brow. I liked that" (Emiri-patient, field notes, p.27). Emiri, talked about her initial response to meeting nurses for the first time, how she could tell within a couple of minutes whether she was going to get along with them and whether they judged her by her size. She did this by watching how staff reacted to seeing her for the first time, how they approached her in the bed and the ease at which they spoke to her (Emiri-patient, field notes, p.25). Unbeknown to Emiri and Gary, many of the nurses who cared for them, including Ella, expressed prejudice towards fat people and caring for fat patients.

Staff regularly concealed their private emotions and feelings when engaged in bedside patient care: "She didn't ever say that to the patient because you just wouldn't. I can see how easily it would be to think that you might want to say something when you get frustrated" (Glenda-nurse, interview, p.8). Staff had concealed their emotions when caring for Emiri. Her pain and discomfort had been challenging for the nursing staff to manage due to her chronic back pain and ill-fitting chair and had required many assisted position changes in bed or from bed to chair and vice versa each hour. She had become more and more irritable about being uncomfortable despite the immediate response by the nurses to her requests for assistance. Towards the end of the shift Emiri had complained to her partner that she wasn't happy with the nursing staff; that they hadn't done enough to get her comfortable (Emiri-patient, fieldnotes, p.17). Hayley had overheard these comments but nevertheless continued to respond to Emiri's requests with the same kindness and friendly demeanour that she had done all shift. Once in the staff room Hayley had vented her real feelings to a colleague: "she said she felt like standing in front of her and saying look what you've done to yourself. You can't even help to reposition yourself in the chair and you expect the nurses to be able to do it" (Glenda-nurse, interview, p.8).

Staff concealed their thoughts and feelings from other staff as well as patients. This had been most apparent when interviewing close colleagues and friends. How staff interacted with patients and other staff was not always reflected in their feelings and opinions about fatness. Glenda, was an exemplary role model on the unit, and well respected by all staff and patients in her care however, during the interview she had revealed strong negative feelings about caring for fat patients: 
I look at them [fat patients/ people] really negatively. My husband's told me that. He's like 'god you're hard on fat people you shouldn't draw judgement'...//... I know that I can feel myself thinking negative thoughts.... I do think I'm able to hide that and put it aside to get on with what I need to do (Glenda-nurse, interview, p. $11 \& 13$ ).

This example demonstrates how in the activity of managing 'faces' staff adopted a professional demeanour that represented the health professionals' expected presentations of nursing and medical behaviours whilst at the same time suppressing their real feelings and emotions.

The staff room often provided the location for staff to present their private 'face' and discuss their real feelings with other colleagues. The further away from the patient the more explicit staff were about how they felt: "People feel that they are in a safe place [staff room] where they can really say what they think" (Shirley-nurse, interview, p.10). It was in these staff designated zones that staff spoke most freely about their feelings of anger, frustration, blame, resentment, and repulsion of caring for fat patients. Thus, the actions and feelings expressed during patient interactions did not match the behaviours and opinions of staff in the staff room or during interviews where real private feelings were expressed.

At times staff did discuss fat patients in derogative and demeaning ways within the clinical patient area. This had occurred during the bedside handover of Don who had just been transferred from the operating theatre to the unit. At the bedside the anaesthetist had referred to Don, who was sedated and ventilated, as a 'slob' when discussing his diabetes regime (Don-patient, field notes, p.1). Bedside conversations were more likely to happen amongst staff if there was a novelty factor surrounding an expected admission such as one in which the patient weighed $330 \mathrm{~kg}$ : "On the way to the coffee room...there was a lot of derogatory talking" (Trudy-nurse, interview, pp.9-10),..."To begin with it was a joke" (Vicki-nurse, interview, p.7). The novelty and fascination about these patients led to idle gossip: "On the phone all we got was that she was extremely large, that her apron they removed was large and she needed the fire service to help her mobilise... all unnecessary in the medical handover" (David-doctor, interview, p.8), sharing of rumours: "I've heard whether it's true or not 
the story about... the fire crew apparently had to come and help" (Trudy-nurse, interview, pp. 9-10), and speculation: "if they put them on a ventilator we will never get them off" (Vicki-nurse, interview, p.6).

On occasion staff did reveal their feelings of frustration to their patients during care situations: "I'd made a hell of an effort getting her there [to the shower]...she wouldn't participate. I did get really frustrated and ended up telling her that I was going to leave her for a few minutes to shower herself" (Glenda-nurse, interview, p.11). Staff deemed it acceptable to express their frustration in situations where patients were perceived to be uncooperative: "They'd certainly know in my tone if I was frustrated if I felt that the attitude was unhelpful and if I felt they could be helping and weren't' (Sophie-nurse, interview, pp.10-11).

The use of humour was one way staff managed their feelings: "we [nurses] do like to laugh at people. I think that's part of coping...to get through your shift, if you're struggling to hold a patient because they're so big and heavy you're going to make a joke about it" (Shirley-nurse, interview, p.4). Humour was used to impart a judgement: "oh my dear you need to be introduced to Jenny Craig or gastric banding because this isn't working for you I can tell you from here" (Robyn-nurse, interview, p.8), to describe a body part: "muffin racks" (Julian-doctor, interview, p. 10), or to describe a challenging care situation: "By the end of the nightshift we were referring to freeing Willy [the patient] in room 14. Every time we turned this person we'd have to strap them in with pillows and sheets to prevent them from falling out of the bed" (Robyn-nurse, interview, p.7).

Humour was often contained to staff designated zones such as the staff room and in the staff seminar room where shift handovers took place: "It's not done to the patient's face or within earshot" (Shirley-nurse, interview,p.4),..."but there is still the tea room banter" (Beryl-interview, p.5). Occasionally, this humour seeped into the clinical environment: “sometimes we forget the patients aren't all sedated and actually they can hear everything you're saying even though it's all meant in jest" (Shirleynurse, interview, pp.4-5). In these situations staff were often aware that their private 
'face' presentations were deemed inappropriate at the bedside as demonstrated in the modified behaviour of staff:

That's why you're saying things in hushed tones and that's why you're not being blatantly critical about the person, lots of innuendos, lots of suggestions and silences that you fill the word in yourself. That all implies that you know you shouldn't be, but it's funny so you will (Shirley-nurse, interview, p.10).

\subsubsection{The language of fat}

The use of language to discuss fatness revealed a tension that existed between the presentation of professional and private 'faces'. This was most evident in the usage of the words 'fat' and 'obese' which were the most frequently used descriptors of the fat patient in clinical practice, both at the bedside and in staff designated zones. Staff were divisive in whether they would or wouldn't use the terms 'obese' and 'fat' in practice: "Within earshot of the patient I talk about obesity and I don't generally tend to use the word fat" (George-nurse, interview, p.10), “...I'd use the word fat rather than obese" (Helen-nurse, interview, p.6).

Reasons for why staff favoured 'fat' or 'obese' over the other were related to using correct medical terminology: "I would state that they're obese. It's important to recognise that term because that's what the patient is" (Bob-nurse, interview, 9), own personal feelings: "I'd be mortified [being] described as morbidly obese in the bed, it would be awful so that's why I wouldn't use that" (Vicki-nurse, interview, p.6), own personal interpretations of the terms: "It's actually a lot worse to call someone obese than just fat...obese is more of a dramatic term of saying you're beyond fat and morbidly obese, it's like hammering the nail in the coffin" (Lee-nurse, interview, p.6), the environment: "In a clinical setting I would use the word obese because that's appropriate to the context but if we were talking about other issues I would probably say fat" (John-doctor, interview, p.13), not wanting to appear judgemental: "I don't think I'd use the term fat. I think that is extremely judgemental" (Bob-nurse, interview, 9)... "Obese has a big subjective element to it. It's not just passing on information, it's actually passing on judgement" (David-doctor, interview, p.4-5), and being conscious of society's usage of the terms: "We are generally uncomfortable of the societal associations of the word obese [and] the associations that people have 
with obese people. Once you use that label then the patient may find it offensive" (John-doctor, interview, p.6). Patients, themselves, disliked the word 'obese': “A friend of mine had called a patient obese in her nursing notes and the patient read the notes and was massively offended by it" (Florence-nurse, interview, p.8). In conversations with Gary he stated that he didn't mind being called 'the big fellow' or 'fat guy' but really hated and was offended by the word 'obese' (Gary-patient, field notes, p.13).

Despite the disagreement in the use of the words 'obese' and 'fat' staff maintained acceptable clinical language through modifying and regulating their conversations. The proximity of staff to the patient influenced the language and types of observed conversations that occurred between staff. Certain words and phrases were deemed appropriate for bedside conversations, staff designated zones such as the staff room and shift handovers, and outside of the workplace.

At the bedside in the presence of fat patients the terms obese, high or increased BMI, bariatric, fat and overweight were used between staff. The choice of these commonly used clinical bedside terms were further sanctioned depending on whether the patient was conscious or sedated and ventilated. When the patient was awake and within hearing distance staff appeared to take even more caution with using language that included 'fat' and 'obese':

When they're awake people [nurses] are embarrassed to say this person is morbidly obese. You don't want to turn round and say 'oh this woman's morbidly obese or they're a little bit fat'. You don't want to ruin your relationship that you've built by saying that (George-nurse, interview, p.8).

Cryptic communication practices were also used: "she's quite round underneath the sheets... or quite big or quite a bit of extra padding there" (Carolinenurse, interview, p.9) or euphemisms such as referring to the need for additional man power were used: "You tell them [staff] how many nurses it takes to manage doing turns and things like that. It's a way of skirting round the issue but making the point" (Molly-nurse, interview, p.4). When nurses were in direct conversation with patients staff avoided making reference to fatness and focused on the challenges of body size: 
"I would not say the reason we can't move you is because you're obese. I would say because of your body size or because you're unable to do it yourself or because you're really sick" (Bob-nurse, interview, p.10).

At times, staff used the patient's own language of fatness to frame their conversations: "If the patient says it first [fat] then I guess it's probably okay to use it" (John-doctor, interview, p.12). This was observed in the care of Gary who was very explicit to staff regarding his preference for using 'fat' in conversations when referring to his size. However, staff remained cautious in their use of language: "It might be okay for them to use it [fat] but not okay for you to use it when talking about them" (John-doctor, interview, p.12). To mitigate the uncertainty of the patient's reaction to the use of the terms 'obese' and 'fat' staff regularly replaced these words with euphemisms.

Euphemisms were used regularly in the presence of conscious patients and became a polite way of identifying that the patient was fat during bedside handovers and medical ward rounds. Frequently used euphemisms for 'obese' and 'fat' were big people, a bit chubby, very large, quite big, pretty heavy, pretty big, heavy, huge, quite heavy to move, tubby and cuddly. Additionally, more descriptive euphemisms such as 'adipose tissue to spare', 'well endowed', and 'excess soft tissue' were used to emphasise the severity of the patient's fatness.

The nature of the language was more derogative when out of hearing of patients. In staff designated zones participants were more at ease discussing and describing the patient: "[I'm] much more comfortable with using words like 'obese' or 'fat' or even something like 'it's just gross that they've let themselves get that big' and talk about fat rolls and aprons" (Glenda-nurse, interview, pp.8-9). Staff were freer in their elaboration of the appearance of fat bodies: "certainly we are a little bit more explicit in the words we use in the staffroom" (Shirley-nurse, interview, p.11). In particular, this was most noticeable when staff described the physical appearance of the patient's fat body where terms such as 'chicken wings', 'big apron', 'no neck', 'fatdupadubs', 'muffin racks', 'shelves', and 'big tummies' were commonplace words. Similarly, special terms and phrases such as 'muffinectomies' and 'apronectomies' 
(Julian-doctor, interview, p.10) were created by staff to describe the procedures of removing excess fat tissue.

\subsubsection{Social awkwardness}

Social awkwardness was discussed by staff and observed in their interactions with fat patients. Staff were conscious of this awkwardness between them and the fat patient: "We do tread on eggshells about everything" (Phillippe-nurse, interview, p.4), "society has made us treat them [fat people/ patients] differently" (Milly-nurse, interview, p.4). Staff felt uncomfortable mentioning or acknowledging the patient was fat in their presence and identified feelings of discomfort, uneasiness and apprehension when engaging with fat patients: "I don't think I would really be comfortable talking to a patient about their weight... I would really struggle to have that conversation with a patient" (Cathryn-nurse, interview, p.4)..."we battle with our own feelings about the fact that we're not comfortable" (Ruth-nurse, interview, p.4). This awkwardness resulted from the awareness of the social tensions surrounding fatness where individuals had concerns of personal prejudices being exposed: "I don't want them to think that I've got some prejudice against them that's going to influence my care" (Glenda-nurse, interview, p.8), being associated with mainstream societal views: "We're part of society. We're not above it" (John-doctor, interview, p.8),..."[the] hospital's a reflection of society" (Bob-nurse, interview, p.6), and acknowledging the patient's own sensitivities: "people assume that the patient will have a degree of sensitivity about the word [fat] or about themselves being overweight" (Rebecca-nurse, interview, p.8)..."They're very embarrassed of their size so you don't really want to accentuate that" (Helen-nurse, interview, p.5). For these reasons staff often struggled to have honest conversations with family about the impact fat had on the patient's condition:

I had a meeting about withdrawing care...it wasn't the small operation she'd had, it was the advanced heart failure because of her weight. The family were saying well what's wrong with the heart?... I couldn't bring myself to say that this is all because she was fat...I didn't want to be seen to be judging her (David-doctor, interview, p.3). 
To avoid verbalising the patient's fatness, staff spoke about and were observed using secret codes or gestures in their clinical practice. During clinical bedside handovers many nurses consciously chose not to directly disclose information related to the patient's fatness: "If you're at the bed space and can see the patient it's pretty self-explanatory" (Jane-nurse, interview, p.6), or they engaged in a culture of muttering: "We sort of might whisper and go 'they're rather large'...[I] don't want to be saying things out loud even if they're sedated" (Jackie-nurse, interview, p.4), secret codes: "We have all those codes [euphemisms] don't we for obesity or high BMI or bariatric" (David-doctor, interview, p.3), and non-verbal gestures: "With a wake patient I'd get the ICU admission note where they list the past medical history... I'd be pointing at it [increased BMI] without saying anything" (Ella-nurse, interview, p.6). This practice of pointing and circling the words that indicated the patient was fat was the most common way of discreetly drawing the nurse's attention to the information pertaining to fatness. During the bedside hand over of Emiri, Hayley had read off the ICU admission notes. As she read the report Hayley paused when she got to the list of co-morbidities and carefully selected some to verbally mention. At the same time she tapped the words 'increased BMI' with her index finger. Ella nodded in acknowledgement and Hayley moved on with the rest of the handover (Emiri-patient, field notes, p.22). This type of behaviour of subtly drawing attention to the patient's fatness was observed in the care of other fat patients.

This practice was not shared by all staff. Some nurses did not avoid discussing fatness with patients and families, and felt that this was part of normal healthcare practices: "I'm more than happy to discuss their size with them... and point them in the right direction" (Lee-nurse, interview, p.5). Staff were more likely to discuss issues of fatness if they felt the reason for the admission was secondary to their fatness: "If they've had a heart attack or they've come in for surgery, you know weight related problems then I'd definitely talk to family about lifestyle changes. If the person's awake I'll definitely talk to them about it as well" (Vicki-nurse, interview, p.11). Equally, staff that were comfortable discussing fat issues with patients did not engage in the unit culture of non-verbal gesturing and recoding of the word 'obese': "Okay [others would say] suffers from hypertension, cholesterol, morbid obesity and 
will turn the other way so that they can't hear... they need to hear it" (Lee-nurse, interview, p.6).

These different approaches to addressing fatness amongst the staff in the presence of the patient only accentuated the social awkwardness present at the bedside. This awkwardness had occurred during the bedside handover of Gary, when Rose had commented that Gary was "morbidly obese which you can see for yourself" in a voice intended for the patient to hear. Helen appeared embarrassed and uncomfortable as she glanced up to establish if Gary had heard (Gary-patient, field notes, p.26).

Social awkwardness was present between staff of different sizes. This was most prominent in the staff room where many staff presented their private 'face' feelings about fatness: 'I'm sure they do [take offence], I don't think I've been there when someone's been offended outwardly. If they have been offended then they've not said anything" (Shirley-nurse, interview, p.5). Staff responded in different ways to the presence of a fat staff member when joking about fatness or a particular patient: "Sometimes people look at me and they will be quite quiet. Other times people would just look at me and expect me to join in. It's very uncomfortable" (Rita-nurse, interview, p.8). One way fat staff often managed the conversations to minimise social awkwardness was through directly addressing the issue of their fatness by making jokes about themselves: "I have to be careful of my rolls, it's taken me years to perfect these" (Rose-nurse, interview, p.11),..."I'm actually bigger than them so what does that make me?" (Shirley-interview, p.5). This approach was observed on numerous occasions and appeared to readdress the interpersonal tensions present and restored social unity. Other fat staff choose not to take any notice of the conversations: "I think that, they've probably got some issues as well and I tend just to step back and just ignore it" (Julian-doctor, interview, p.11).

Staff were aware of the awkwardness of discussing the fat patient in the presence of fat staff and changed their behaviours during direct patient care: "When we are turning a big person, and there's other nurses helping me who are really big that's when I really feel uncomfortable and you know really want to watch what I'm saying" (Stella-nurse, interview, p.16), and when discussing fat patients in the staff 
room: "I'm aware that several of the staff are actually very sensitive about their weight and I'm trying not to use these phrases [derogative words] in their earshot" (David-doctor, interview, p.11). These changes in behaviours when engaging with fat patients and fat staff only served to accentuate the social awkwardness that was present.

\subsubsection{Mutual Pretense}

Mutual pretense was used by staff and fat patients as a way of managing the social awkwardness. There was an awareness that everyone knew the patient was fat including the patient: "They know that you know that they are overweight" (Sophienurse, interview, p.5). Yet conversations about their fatness was avoided at the bedside: "No one wants to talk about the elephant in the room. There's something huge happening but no one wants to talk about it" (Vicki-nurse, interview, p.4),..."You're all faced with an obese patient but no one wants to say but this patient's obese" (John-doctor, interview, p.6). By not acknowleding the patient's fatness staff entered into a pretense that the patient's fatness did not exist. This allowed for the issues of fatness to be ignored as staff worked to manage the social awkwardness of discussing fatness. Thus, actions and conversations that would openly expose the patients' fatness were avoided: "It's not a topic that I'll bring up with patients. I don't know whether they'd want to talk about it or not" (Maggie-nurse, interview, p.10). Types of conversations avoided with patients were those that directly addressed their weight: "We're not proactive with talking about obesity with patients" (Cathryn-nurse, interview, p.4), or identified the patient as being fat: "I don't actually mention they're obese [in bedside handover]...I wouldn't necessarily mention it at all" (Mary-Anne, interview, p.11).

During this pretense staff pretended not to notice the patient was fat: "I don't acknowledge to the patient that they're obese" (Stella-nurse, interview, p.10), or that they were difficult to move around the bed or into a chair: "I would not say the reason we can't move you is because you're obese I would say because you're really sick we need more of us to move you" (Bob-nurse, interview, p.10). On the many occassions when fat patients did not fit into the equipment properly neither staff or patient acknowledged this fact and both continued on as if nothing was amiss. 
The rules of the pretense changed if the patient mentioned their weight or size. In these instances staff primarily attempted to block the patient's effort at openness by denying the patient had a weight issue: "if someone goes 'oh I'm fat', you go 'oh no you're not'...you don't want to say that they're fat" (Kate-nurse, interview, p.6)...'I work harder to act like it's all normal" (Stella-nurse, interview, p.12), or minimised and directed conversations away from fatness: "I would probably try and minimise it [the conversation]. I don't want to deal with it" (Stella-nurse, interview, p.10). By engaging in these strategies staff attempted to re-establish the pretense.

However, some patients, in particular Gary, made their fatness a focus of many conversation with staff. He was always joking about fatness, making comments about other fat staff that he saw walking past his bed space and questioned staff about their own perspectives. This open acknowledgement about the willingness to discuss fatness appeared to lessen the awkwardness and tension in the observed body language when fatness was mentioned in front of him. However, staff appeared to where possible maintain the pretense by not initiating conversations, only responding to patient directed conversations and continuing to engage in indirect communication with colleagues during shift handover.

The delicate balancing act of pretense between the staff and patient was always being threatened by the presentation of the private 'face'. When under intense physical strain or pressure during care this pretense failed and private feelings and behaviours seeped into the clinical spaces of the unit where retaliations to patients about being fat, and inappropriate comments and jokes about their fatness occurred. Mostly this seepage of private feelings occurred in the clinical areas that were not involved in direct patient care such as the nursing station, drug room, and computer spaces. Incidents involved joking on the telephone or making a joke about a patient who was at the other end of unit, or discussing the prospect of a new patient being admitted. In these instances these expressions of private feelings were not directed at the fat patient. However, other staff, and at times other patients and family may have observed these private 'face' judgements. 


\subsection{Chapter summary}

Through the 'situated' experiences of ICU staff this chapter has described the specific ways in which fat patients were considered to be 'misfits' in the ICU. It revealed how the critically ill fat body did not fit physically, medically or socially into the constructs of everyday normal ICU practices. It has revealed how staff managed their personal perceptions of fatness during care situations through professional and private 'face' activity. The findings reveal how the social awkwardness of stigma infiltrated care situations which required staff to engage in specific types of care practices. In the following chapter, I examine the significance of my findings in relation to the professional and private 'face' activity that occurred during the social interactions and care of fat patients. This discussion focuses attention on how staff managed their private and professional 'face' through the use of emotional labour, behavioural regions and face-work to reduce the social awkwardness present during care situations. 


\section{CHAPTER SIX: DISCUSSION}

\subsection{Introduction}

This study aimed to explore the culture and influences within the intensive care setting in which nurses and doctors provided care to extremely fat patients. A focused ethnographic methodology was used to study one intensive care unit in New Zealand. Data was generated during four months of fieldwork via participant observation, interviews and review of cultural documents and artefacts. Data were managed using Nvivo 9 software and analysed using conventional qualitative techniques. In this chapter I present the key findings of the study and provide a critical discussion of how these findings support previous research, theoretical understanding, and add new insights to the body of knowledge on how fat patients are perceived and cared for within the intensive care setting. The first key finding was that fat patients were 'misfits' within the ICU setting. The concept of 'misfits' referred to how the fat patient did not fit the physical, medical and social norms of intensive care practices. The second key finding was how staff managed their private perceptions of fatness during care situations through the use of emotional labour, behavioural regions, and face-work. This included showing how the tensions between private and professional 'face' performances of staff created social awkwardness for both staff and patients during care practices.

\section{2 'Misfits'}

Within the ICU there were care challenges relating to the 'fit' between the patient and the medical environment. In particular, there was often an issue between the size of the patient and the size of the equipment used during care; this occurred when the patient was too large and the equipment too small. Similarly, the inconsistency of how fat was distributed in the bodies of fat patients in ICU meant that patients did not always fit with the specifically designed equipment: this created a physical 'misfit' of design between the fat patient and the equipment in ICU. Factors that impacted on the fat patient's inability to fit into their physical surroundings led them to also becoming a social misfit. 
This study has demonstrated that within ICU there was a 'misfit' between the fat body and normal medical practices due to the changes in the fat patient's anatomy and physiology. These changes were irrespective of the underlying pathology (e.g. cardiac disease, sepsis, respiratory failure) and the reason for admission. To date, the concept of 'misfit' or issues of 'fit' has received little attention in the medical literature on the management of fat patients. Whilst there is clear exploration in the medical literature about management of specific issues such as difficult and prolonged ventilation and the difficulties in gaining vascular access in fat patients, this is solely discussed through the theoretical lens of altered anatomy and physiology (El-Sohl, 2004; El-Sohl \& Jaafer, 2007; Gross, Cohen, Andersen, \& Wax, 2002).

Only one study by Gross et al. (2002) has specifically identified the fat person's anatomy as creating a issue of 'fit'. Gross et al. (2002) hypothesized that the standard-sized tracheostomy tube fitted poorly due to increased neck fat where the tracheostomy tube was too short and too curved for the fat patient's neck; this increased risk of tube displacement. Two techniques were identified to overcome this problem: modify the tracheostomy tube or surgically removing the neck fat to fit the standard-sized tube. The restrospective review of 23 patients focused on assessing the safety and morbidity of the 'defatting' tracheostomy technique to which Gross et al. (2002) they concluded was the preferred option in making the patient 'fit' the standard-sized equipment. Conceptualising fat patients as medical 'misfits' provides a new focus of learning in understanding the medical management issues of fat patients whilst at the same time reinforcing current knowledge in this area of medical practice.

Physical fit was a challenge for fat patients in ICU because of the two distinct but interrelated issues of size and design between the patient and the care environment. Issues of size related to the misfit of size between the patient and the standard and bariatric equipment used to manage patient care needs. To date, the subject of the physical fit of fat patients in the hospital setting has not been well explored in the literature. One exception is the study by Merrill and Grassley (2008) who identified that the physical environment of clinics and examination rooms created a misfit of 'size' for fat women seeking healthcare. In their study, fat women did not fit into normal healthcare spaces because of their size and weight. As in the ICU setting, 
standard size and bariatric sized chairs, examination gowns, and other equipment e.g. blood pressure cuffs were found to be too small or ill-fitting.

This new and emerging health need means that there is very little robust empirical work in this area. There are published case studies (Davidson, Kruse, Cox, \& Duncan, 2003; Kells, 2005; Muir, Heese, McLean, Bodnar, \& Rock, 2007), and many opinion articles (Camden-Gallagher, 2009; Trimble, 2007) which have led to non-evidence based best practice guidelines (Hurst et al., 2004; McGinley \& Bunke, 2008). Therefore, this original study of fat patients in ICU significantly contributes to exploring the concept of fat patients and the practical challenges of care in hospital settings.

The second significant issue in ICU, in terms of not physically fitting, occurred as a result of the design of the equipment used. Often the principle of scaling up the equipment was adopted, whereby the chair was made proportionally larger and not specifically designed to attend to the patient's physical needs. For example, the scaling up of the bariatric chair used in ICU meant that in order to accommodate the width of the patient the depth of the chair seat became too deep. Similar design issues were also raised in Forhan et al.'s study (2010) where access to lifts, narrow staircases and stairs, without sufficient depth to every step, were particularly problematic for fat patients. To date, there is limited empirical work that addresses the design of the equipment where fat patients are the participants of the study.

There has been some work conducted outside of the hospital setting by researchers in the field of occupational therapy and rehabilitation, which highlighted issues of 'fit' that reduced a person's ability to participate in activities of daily living (Forhan et al., 2010). Specific issues raised included the size of seating in cinemas, restaurants and on public transport. Forhan et al.'s (2010) work emphasised that the issues of 'fit' were related to the physical size and design of the generic world. 
The concept of physical and social 'misfits' has been explored in GarlandThomson's (2011) work where she explored how fat bodies ${ }^{45}$, were a variation of the normal body (Garland-Thomson, 2005, 2011). In this work it was suggested that there were issues of ' $\mathrm{fit}$ ' between the fat person and the world in which they lived. 'Misfitting' was described as an incongruent relationship between 'two things' and the awkward attempt to make them fit together. Garland-Thomson (2011) argued that the 'generic' ${ }^{46}$ world had been conceptualised, designed and built to meet the specifications of the uniform, standard, body. Therefore having a normal, in this case, slim body, allowed individuals to navigate the world without drawing attention to themselves and therefore were able to fit in (Garland-Thomson, 2011). Fat patients were noticeable in the ICU setting and did not blend into the ICU environment; this caused a social scene ${ }^{47}$. Therefore, fat patients stood out physically and socially thereby emphasising the issue that they did not fit.

This concept of physical and social misfitting is further explored in GarlandThomson's (2011) work where she identified that there were spatial (physical) and temporal (social) contexts in the generic world in which the body did not 'fit'. The spatial context related to the issues of 'fit' within the physical environment, such as problems encountered in accessing public buildings. Whereas, the temporal context related to issues of 'fit' that occurred as a result of the social attitudes and social exclusion by others (Garland-Thomson, 2011). In this respect, both spatial and temporal contexts of 'misfitting' share similarities with the concepts of physical and social misfits within this ICU work.

Fat patients as social 'misfits' was a particular area identified in the ICU setting that affirmed the current literature describing the perceptions of healthcare professionals for why people are fat (Culbertson \& Smolen, 1999; Hoppe \& Ogden, 1997; Poon \& Tarrant, 2009), and why they do not meet social expectations (Hoppe \&

45 Garland-Thomson's $(2005,2011)$ work explored the broad concept of disability where she identified fatness to be a physical disability. It is these broad concepts of her work that have been drawn on in this discussion.

46 Garland-Thomson (2011) referred to the 'generic' world as a place which had been designed to accommodate the dominant majority body, i.e healthy normal weight bodies

${ }^{47}$ Social scene in this context refers to the obviousness that the person did not fit their surroundings 
Ogden, 1997; Mercer \& Tessier, 2001). ICU staff percieved patients to be fat because they ate too much and did not exercise, and did not meet social expectations of taking responsibility for their own health. A misfit of 'behaviours' in terms of 'expected' and 'percieved' behaviours has been highlighted in a study by Creel and Tillman (2011) who explored the stigmatisation of fat patients by nurses. Fat patients reported that nurse made assumptions that they did not exercise, were unclean, unhealthy and that their illnesses were related to their fatness. ICU staff perceptions of fat patients supported Creel and Tillman's (2011) assertions of fat patients' 'percieved behaviours' which influenced their judgements of fat patients.

There is also evidence of fat patients perceiving themselves as 'misfits' within the social world and healthcare system (Creel \& Tillman, 2011; Forhan et al., 2010; Merrill \& Grassley, 2008). In this body of work, it is clear that the perceived attitudes and behaviours of others towards fat people affected the fat person's sense of fitting in. This concept of 'Battling to fit in' was a theme identified in Merrill and Grassley's (2008) study of fat womens' experiences of healthcare. In this work, fat women reported the constant battle they faced to be accepted as being 'worthy' by healthcare professionals. During social interaction, fat women struggled with being dimissed, disrespected, not being believed, not being quite human, and inferior to thin women, because they did not 'fit' cultural expectations of slimness. Likewise, in Forhan et al.'s (2010) study, fat people perceived that in professional and social situations that their social value and credibility, was negatively affected by being fat. The perception of fat people not being 'worthy' or 'credible' individuals in these studies may somehow reflect the judgemental and disapproving attitudes disclosed in private amongst ICU staff.

The findings that fat patients were 'misfits' in the ICU supports the physical and social understandings of not fitting within the existing literature. Furthermore, it provides insights into the possibilities of additional dimensions of 'misfit', such as medical 'misfits', in which the fat body does not fit within the current healthcare system. Given the demographic changes outlined in Chapter One, it is suggested that the areas raised here are important and need serious empirical consideration. 


\subsection{Managing 'misfits' through professional and private 'face' activity}

ICU staff concealed, modified and regulated their feelings and emotions regarding fatness through the presentation of two different 'faces': the professional and private 'face', where the social 'face' presented was dependent on the 'copresence' of individuals (Goffman, 1959, 1963a, 1967). The condition of 'copresence', which was necessary for all interactions, Goffman (1963a) proposed occurred whenever a person sensed that they were in close enough proximity to be percieved in their activities by others, and likewise when they could sense the activities of others. Within ICU, the 'face' presented was specifically dependent on the co-presence of different groups of people such as other staff, fat patients, families and visitors. The professional 'face' was observed to be the 'caring face', where staff displayed pleasantness, empathy, and kindness towards fat patients during care. This 'face' presented a display of feelings, emotions, and behaviours performed in accordance with the health professionals' expected presentations of nursing and medical behaviours. ICU staff used professional 'face' presentations as a way of concealing what they really thought about fat patients during interactions. Underneath this caring façade staff were socially distanced from their fat patients, and provided care that was obligatory and perfunctionary.

The 'face' presentations of ICU staff have been observed in the works of Hochschild (1983), Bolton (2001) and Cain (2012). The professional 'face' of the staff in ICU reflected Hochschild's (1983) descriptions of the public presentation of emotions by flight attendents, as ICU staff presented a nice, pleasant, and caring demeanor that created a sense of being cared for in a safe place. Although, Hochschild (1983) did not define the 'face' presented by flight attendants per se, her distinction made between emotional labour (public commercialised work) and emotion work (private work), are aligned with the professional and private 'face' activities of ICU staff. In both ICU and Hochschild's (1983) work private negative feelings of anger and frustration were separated from expected public displays of emotions, such as niceness.

The professional 'face' of ICU staff resembled both the professional and smiley 'faces' identified in Bolton's (2001) study of nurses working in a NHS Trust 
hospital. Bolton (2001) differentiated 'faces' based on motives that informed the behaviour. Bolton's (2001) professional 'face' was synonymous with nursing values and informed the delivery of care, whereas the smiley 'face' represented customer service values and informed non-clinical housekeeping activities. In contrast, staff in ICU differentiated 'faces' in accordance with Goffman's (1959) behavioural regions where behaviours and activities of staff were determined by the 'co-presence' of different groups of people, such as staff, fat staff, fat patients, families and visitors. Thus, while there are similarities in the 'faces' described by Bolton (2001) and those used by ICU staff, they have been constructed from different conceptual positions; motives and behavioural regions, respectively.

Similarities can be seen in Bolton's (2001) 'smiley face' and Hochschild's (1983) public display of emotions, as the motives of both nurses and flight attendants were driven by the same commercial values of customer service. Both Bolton's (2001) customer service 'smiley face' and Hochchild's (1983) public display of emotions were observed in the professional 'face' of ICU staff in how they engaged with patients to create a positive ICU experience. In this respect, a positive ICU experience equated to customer satisfaction with the quality of care delivered.

The professional 'face' presentations of ICU staff shared similarities with Cain's (2012) frontstage professional 'identity' of hospice workers, where nurses presented themselves during patient care situations as caring, compassionate and serious. Cain (2012) referred to different 'identities' to describe the various 'faces' or presentations of self within social interactions, just as the staff did in ICU in relation to fat patients. Both the professional 'face' and the professional 'identity' was reserved for frontstage encounters where it was important to preserve the professional image of caring work.

The private 'face' of ICU staff was observed to be the 'contemptuous face', where staff displayed judgement, disapproval and humour regarding fatness and the fat patient during interactions with other normal weight staff. This 'face' presentation involved a display of feelings, emotions, and behaviours in relation to fatness and caring for fat patients that exposed their real feelings. The private 'face' of ICU staff 
reflected Hochchild's (1983) descriptions of the private presentation of emotions by flight attendents, as ICU staff expressed real unguarded negative feelings about their fat patients. The humour expressed by ICU staff, as part of their private 'face' presentation, reflected Bolton's (2001) 'humourous face', where shared jokes eased the stress of care, and Cain's (2012) backstage 'professional identity', where staff exhibited humour and detachment from patients. Private 'face' humour, backstage 'professional identity' humour (Cain, 2012), and 'humourous face' (Bolton, 2001), all shared the same common purpose of managing feelings, maintaining team cohesion and acknowledging challenging care situations amongst other colleagues.

\subsection{1 'Face' and emotional labour}

Staff in ICU engaged in emotional labour as a means to construct professional 'face' performances. Emotional labour was used specifically by ICU staff to hide negative emotions of anger, frustration, resentment, blame, and repulsion so that situations of offense and embarrassment did not occur between staff and fat patients. Emotional labour was seen as an integral tacit process that occurred in order to create and sustain the fat patient's sense of being cared for in a safe convivial place.

The way in which ICU staff concealed, modified and suppressed their feelings of fatness and caring for fat patients aligns with Hochschild's (1983) original conceptualisation of emotional labour. ICU staff, like flight attendants, suppressed their own private negative feelings towards others by presenting an acceptable demeanour in accordance with the employers commerically developed 'feeling rules'. Commercial is likened to professional in this respect. For the nurses, these were the codes of conduct that required nurses to treat patients with respect, dignity, kindness, consideration, and without prejudice (Nursing Council of New Zealand, 2012). Likwise, for the doctors their behaviour was governed by a similar set of expected standards to treat patients with respect, dignity, honesty, and never discriminate unfairly or "delay or refuse treatment because you believe a patient's actions have contributed to their condition" (Medical Council of New Zealand, 2013, p. 13). ICU staff used these 'professional feeling rules' to guide their interactions with fat patients. 
What distinguishes this study from other studies that have used emotional labour in the construction and management of different 'faces' (see Bolton, 2001; Cain, 2012; Froggatt, 1998; Li, 2002, 2004; Smith 1992) was the motives, types of stress encountered, and strategies employed in managing care situations. For the ICU staff the need for emotional labour was based on the inherent dislike of fatness and what it represented in society. This dislike created an emotional conflict between private and professional values and beliefs. The types of stress encountered were twofold. Firstly, ICU staff had to work on reconciling the conflict between personal and professional beliefs and secondly, work hard on suppressing and concealing private negative feelings when engaged in caring for the fat patient. Emotional labour strategies were therefore specifically focused on hiding private negative emotions of dislike and judgement during social interactions. This type of emotional labour was specific to the management of prejudicial attitudes of staff during interpersonal interactions.

In much of the nursing research, where emotional labour has been used as a theoretical explanation of caring work, the need for emotional labour was based on emotional connections, intimacy, and attachments made with patients (Froggatt, 1998; James, 1989; Smith 1992, Smith \& Gray, 2001, 2001b), not on dislike or personal and professional conflict, as observed in ICU. In the above studies, types of stresses encountered related to suppressing or concealing feelings of despair, failure, and anguish during intensely emotional situations that often involved death and dying. In these situations, emotional labour strategies were focused on protecting the nurse from the emotional harm and burnout of over-involvement and closeness by employing various distancing techniques (Froggatt, 1998; Smith 1992). This type of emotional labour was considered universal to professional caring work.

How ICU staff modified and presented different emotions and behaviours has provided new insights into how emotional labour was used to construct the private and professional 'face' as a mechanism to conceal prejudice and conduct care in a non-discriminating manner. Emotional labour therefore, assisted staff in providing care that was observably indistinguishable from the care provided to other patients. 


\subsection{2 'Face' and behavioural regions}

ICU staff used behavioural regions to inform which 'face' they presented in the presence of others. When in close proximity to fat patients or engaged in patient care staff presented a professional 'face', whereas in the staff room away from fat patients staff presented their private 'face' feelings. The frontstage was synonymous with the professional 'caring face' presentations that portrayed health professionals expected behaviours as outlined in the professional codes of conduct by the nursing and medical professions (Medical Council of New Zealand, 2013; Nursing Council of New Zealand, 2012). Whereas the backstage was associated with the private 'contemptous face' presentations of staff that were more in keeping with mainstream societal perspectives of fatness (Puhl \& Brownell, 2001; Teachman et al., 2003).

Behavioural regions in ICU were determined, not by the demarcation of the physical location of performances as seen in the works of Cain (2012), Coombs (2004), Lawton (1998, 2000), and Tanner and Timmons (2000), but instead by the existence of a 'co-presence' between different groups of people within the same physical setting. Staff managed their different 'face' presentations within the physical environment of the ICU in accordance with Goffman's (1959) condition of 'copresence' which was necessary for all interaction and has provided new insights into when different 'faces' were presented.

The frontstage, where professional 'face' performances were expected, occurred in situations where there was 'co-presence' of staff and fat patients or normal weight staff and fat staff. Professional 'face', or frontstage performances occurred whenever a staff member sensed that they were in close enough proximity to be percieved in their activities by fat patients and fat staff, and likewise whenever they could sense their actions or presence. In contrast, the private 'face' or backstage behaviours occurred whenever a staff member percieved that their activities could not be sensed by fat patients or fat staff or that they could not sense their activities. Backstage private 'face' performances commonly occurred in the 'co-presence' of like-minded normal weight staff. These backstage behaviours, or private 'face' performances, were therefore observed in the clinical zones of the unit in what had been traditionally considered frontstage regions of the hospital (Coombs, 2004; 
Tanner \& Timmons, 2000). Likewise, when a fat staff member was 'co-present' in typically considered backstage regions such as the staff room, seminar room and office spaces, the behaviour observed was that of the frontstage where staff presented their professional 'face'.

What differentiated ICU from other clinical areas of the hospital, which made 'co-presence' more influential than physical setting, was the consideration that ICU patients were usually sedated and ventilated during periods of their admission. Access to the unit was highly restricted both in terms of times to visit and numbers of visitors, and the physical structure and layout of each bed space isolated social interactions to those in the immediate locality. Each of these factors affected the social conditions necessary for 'co-presence'. Therefore, within the clinical zones of the ICU there was very often an absence of 'co-presence' between staff and fat patients or fat staff.

The term 'co-presence' has received little attention in the nursing literature, however it was implicitly considered by Fleischer, Berg, Zimmermann, Wüste, and Behrens (2009) to be an important component of nurse-patient interactions. Similiarly, Oliver and Redfern (1991) referred to interaction as an observable behaviour which supported Goffman's idea that interaction occurred in situations where individuals could sense the behaviours of others. Most commonly, the term 'co-presence' has received much critical consideration in understanding virtual relationships in the era of the internet where the physical presence of social interaction was absent (Subramaniam, Nandhakumar, \& Baptista, 2013).

The observation of staff caring for fat patients in ICU shares some similarities with the work of Tanner and Timmons (2000), where the operating theatre was considered a backstage region when the patients were anaesthetised. In this sense, the theatre staff used 'co-presence' as the determining factor of when the operating theatre switched 'stages'. However, Tanner and Timmons' (2000) conceptualisation of the operating theatre as a backstage remained premised on the physical demarcation of different 'stages', as demonstrated by their articulation of behaviour changes of staff when working in other areas of the hospital. 
Behavioural regions co-existed in the same physical spaces of the ICU. This concept of multiple behavioural regions existing in the same place at the same time has been explored by Bolton (2001) when describing the circumstances in which nurses presented their different 'faces'. Bolton (2001) referred to this ability to simultaneously present two 'faces' during care situations as a 'double stance', a term used by Goffman (1961b) to describe how the "simultaneous multiplicity of selves" (1961b, p. 132) was presented during official performances. Goffman (1961b) suggested that when a particular definition was in charge of a situation, in this case the professional 'face', other counter-activity (private 'face' activity) could be possible as long as it did not overtly threaten or blatantly reject the official situation. Thus, actors could "introduce a margin of freedom and maneuverability, of pointed disidentification" (Goffman, 1961b, p. 133), as long as the prevailing definition of the situation dominated the performance. Individuals' therefore conformed to the official definition of the situation whilst their gestural activity simultaneously and nonintrusively indicated otherwise. In this way, as long as the official situation dominated and was not challenged, other role identities could be concurrently sustained.

The idea of a double stance has provided a further way of understanding behavioural regions and presentations of different 'faces' in the same physical setting where the 'co-presence' of staff and fat patients existed. For example, staff could maintain the professional 'face' to the patient whilst at the same time incorporating innuendos, euphemisms and non-verbal gestures that signaled to other staff their private 'face' feelings. In this way, staff presented the official professional performances whilst indicating that they were not solely defined by this official professional stance.

While Bolton (2001) described the many 'faces' of nurses in terms of motives, my observation of staff caring for fat patients in ICU has differentiated 'faces' in terms of professional (behaviours seen by the public) and private (behaviours seen only by like-minded colleagues) presentations. Thus, in ICU the professional 'face' reflected both Bolton's (2001) professional and smiley 'faces', and Hochchild's (1983) public (commercial /customer service) displays of emotions to produce a performance fit for Goffman's (1959) frontstage. Whereas the private 'face', reflected Bolton's 
(2001) 'humourous face', and Hochchild's (1983) private displays of emotion were ordinarily contained to the restricted areas of Goffman's (1959) backstage. This use of 'co-presence', as a social cue for determining which 'face' could be presented, has both challenged and extended Goffman's (1959) notion of behaviours being located in specific physical spaces, as determined by the restricted access of certain groups.

\subsection{3 'Face' tensions and social awkwardness}

There was a social awkwardness that existed between ICU staff and fat patients during care situations. For staff, awkwardness presented itself on immediate contact with fat patients, due to the social sensitivities surrounding fatness whilst attempting to determine the boundaries of acceptable acknowledgement of the patient's fatness, or in response to a colleague's indiscretion in the patient's presence. The social tension and awkwardness was most noticeable when the patient's physical differences of fatness could not be ignored by staff, which led to an uncertainty of how to interact with the fat patient, or when staff deliberately drew attention to the persons' fatness in a face-threatening manner.

The sensitivities surrounding fatness during interactions shared similarities with the work of Brown and Thompson (2007) who identifed that the awareness of fat stigma and the psychological and physical impacts of fatness caused social awkwardness during nurse-patient consultations. However, during interactions how awkwardness presented itself and the factors that affected the degree of awkwardness differed between those of Brown and Thompson's (2007) and my findings. Brown and Thompson's (2007) study related to social awkwardness that presented itself during consultations where weight management advise was given. The degree of awkwardness was affected by the primary purpose of the consultation, the level of educational preparedness, the presence of weight management protocols, the perception that it was difficult to achieve change or help the patient lose weight, and how the trusting relationship was negotiated when patient's weren't always truthful (Brown \& Thompson, 2007). Equally, the body size of the nurse affected the degree of social awkwardness, with an amplification of awkwardness felt by slim nurses who were concerned about not appearing empathetic or authentic. In contrast, the degree of social awkwardness in ICU was based primarily on the tension created by avoidance 
of the fat discussions and the need to manage an indiscretion if it occurred and not on how staff engaged in weight management discussions.

In Clegg's (2012a, 2012b) development of a self-regulatory model of social awkwardness he identified that 'social novelty' (i.e fatness) and drawing explicit social attention to a situation (i.e the fat patient being unable to fit onto the commode) increased feelings of social awkwardness. Fatness, as a 'social novelty', and the subsequent feelings of awkwardness within social interactions, shared similarities with other physically observable conditions, such as patients with chemotherapyinduced hair loss (Power \& Condon, 2008), and facial disfigurement (Rumsey \& Harcourt, 2004). In both these conditions there was a percieved threat to body image and altered self-perceptions which led to social anxiety, social avoidance and feelings of shame and embarrassment when interacting with others (Power \& Condon, 2008; Rumsey \& Harcourt, 2004).

Awkwardness was defined by Clegg (2012a) as a sense of moral or social indiscretion that amplifies social experiences by focusing attention on the social behaviours of those involved. During these moments feelings such as tension, discomfort, anxiety, and embarrassment was experienced by individuals (Clegg, 2012a). These feelings were socially expressed and observed as anxious, hesitant, or disjointed performances. These types of feelings and social expressions of awkwardness were evident during care situations amongst staff and fat patients in ICU when a social indiscretion had occurred or when staff attempted to minimise situations that would knowingly cause awkwardness.

Social anxiety has been identified as a precursor or a concomitant to every awkward situation between non-stigmatised and stigmatised individuals (Hebl, Tickle, \& Heatherton, 2000) . From personal accounts of awkward moments, Hebl et al. (2000) suggested that the non-stigmatised person held preconcieved ideas that the interaction would be extremely difficult or negative because it involved interacting with a stigmatised person. This expectancy of awkwardness only served to increase the anxiety which often led to confirmation of this expected awkwardness. The causes for the anxieties felt by ICU staff when engaging with fat patients shared similarities 
with those described by Hebl et al. (2000) between non-stigmatised and stigmatised individuals. These were: violation of norms and expectations of interaction, thought suppression, misrepresentation, and approach-avoidance mechanisms.

In situations where a violation of norms and expectations of social interaction had occurred, Hebl et al. (2000) identified that anxiety existed because the nonstigmatised person was unsure how to act and became self-conscious of the restriction imposed on their normal range of verbal and non-verbal responses. This restriction required them to monitor their word choices and non-verbal behaviours. This anxiety, or hesitant disjointed behaviour, was observed in the way ICU staff restricted their bedside language, used euphemisms and engaged in secret non-verbal gestures. As well as not knowing how to act, non-stigmatised individuals tried not to say the wrong thing, although, often this meant that they did not know what the right or wrong thing was. Therefore, like the ICU staff, the non-stigmatised person attempted to suppress all thoughts concerning the stigma by avoiding all topics of conversation that vaguely related to it (Hebl et al., 2000). In addition to thought suppression, many nonstigmatised individuals were found to avoid certain topics or interactions altogether because of the belief that the stigmatised person would be overly sensitive about their stigma and offended if topics related to the stigma were discussed (Hebl et al., 2000). These assumptions and behaviours were evident in the way that ICU staff talked about and interacted with fat patients during care situations.

A further cause of anxiety for ICU staff was the concern about revealing private 'face' feelings during interactions with fat patients. This heightened social anxiety has been identified in other situations where the non-stigmatised person holds neither solely negative or positive feelings when engaging with a stigmatised person (Hebl et al., 2000). In these circumstances, an approach-avoidance mechanism is said to occur as the non-stigmatised person is attuned to societal norms to be caring towards the stigmatised person during interactions, however, at the same time they attempt to avoid interacting with them for fear of personal exposure of their real feelings (Hebl et al., 2000). 
The principle that underpinned the concept of social awkwardness in Clegg's (2012b) model was 'acceptance' within the social functioning of the group. Any threat to this acceptance within the social interaction created awkwardness. During interactions with fat patients there was always a potential threat to 'acceptance' due to the social difference, disapproval and moral judgement expressed in private by staff, which could be revealed during interactions. Circumstances that increased awkwardness included non- and counter-normative situations and behaviours (Clegg, 2012b; Hebl et al., 2000), negative social judgements (Clegg, 2012b; Hebl et al., 2000), and making explicit the social processes of interaction (Clegg, 2012b). These types of situations were evident in ICU and have provided new understandings of why social awkwardness presented itself in specific ways between normal weight staff, and fat patients or fat staff.

Managing socially awkward moments during care situations (through prevention or resolution) was identified as a fundamental component of maintaining or re-establishing positive social interactions between staff and fat patients in ICU. The strategies adopted to prevent or resolve awkwardness shared similarities with those decribed by Clegg (2012a) of avoidant or direct behaviours. Avoidant behaviours blocked direct communication about the indiscretion that occured or involved distancing behaviours that avoided future encounters. Whilst Clegg's (2012a) strategies of avoidant behaviours, such as avoiding all conversations about fatness or pretending not to notice the patient was fat, were employed by ICU staff to resolve social awkwardness, they were further used as a means to prevent awkward situations from occurring in the first instance. These avoidance behaviours can be likened to what Goffman (1967) referred to as face-saving acts. Behaviour changes of this nature, Clegg (2012a) proposed, only served to "entrench the very awkward feelings and interactions being avoided" (p.272) and prolonged the social tensions to future encounters.

Direct acknowledgement of the awkwardness of the situation, primarily through humour, was considered the most effective and immediate strategy of interpersonal resolution (Clegg, 2012a). Humour was employed by fat staff and patients as a strategy to ease and diffuse the social tensions in such situations. These 
direct responses re-established "a sense of social harmony" (Clegg, 2012a, p. 273). Whilst humour has been used to explain the diffusing of awkward situations, it has also been identified as an important precursor signal to awkwardness (Sparks, Travis, \& Thompson, 2005; Sparks-Bethea, Travis, \& Pecchioni, 2000). In other words, humour was employed by an individual to signal to others that they were approaching topics of conversation that involved socially awkward experiences. In the research by Sparks-Bethea et al. (2000) and Sparks et al. (2005) social awkwardness related to describing the intimate details of caring for a dependent older family member. In their studies, the disguise of humour was considered to be a safety-valve that relieved stress, tension and embarrassment and served as a face-saving act in communication of percieved sensitivity.

The findings of this study extend understandings of social awkwardness in the context of fatness and caring for fat patients in two ways. Firstly, social awkwardness occurred due to the presence of a pre-existing "latent social tension" (Clegg, 2012a, p.272), and secondly, because moral and social boundaries had been disrupted (Clegg, 2012a). Latent social tensions were the awareness of fat stigma and the knowledge that fat patients were 'misfits' and did not fit into the space of the ICU. This preexisting knowledge affected social interactions, where staff engaged in avoidance strategies which at times accentuated the awkwardness experienced. Secondly, social awkwardness occurred because the physical fat body represented a latent moral disruption (or weakness) of the person's character which was visible throughout the social interaction in the form of fat (Hebl et al., 2000). Thus, their physical presence and 'social novelty' routinely made interactions awkward.

Another area where social awkwardness occurred during care situations originated from within the intensive care team. Social tensions and awkwardness arose when ICU staff used language that other staff might find potentially offensive, spoke directly with fat patients about their weight, or did not adhere to the secret codes and behaviours of the official frontstage. These inconsistent team performances created social awkwardness between staff in the presence of fat patients. In terms of Goffman's (1959) impression management, this awkwardness was present because individual staff were viewed to have directly violated important dramaturgical 
principles of loyalty and discipline which disrupted the whole performance and line maintained. Dramaturgical loyalty, Goffman (1959) proposed, was about maintaining a strong cohesive team and required team-mates to behave in certain ways to maintain the team's agreed performance. Whereas, dramaturgical discipline required teams to have "presence of mind" (Goffman, 1959, p. 210) and "self-control" (Goffman, 1959, p. 211) to be able to suppress their negative reactions to situations. Staff indiscretion and revealing backstage private 'face' secrets of the team challenged the dramaturgical loyalty of behaving in certain ways to maintain the agreed professional 'face' performance. Similarly, not suppressing one's own negative reactions and engaging in face-threatening interactions questioned the individual's dramaturgical discipline of self-control. When this happened the situation had the potential to become an awkward incident where back region thoughts and emotions were exposed.

\subsubsection{Face-work}

Social awkwardness, as a result of 'not fitting' into the space of the ICU, was managed by staff through specific types of face-work to address potential issues of embarrassment, humiliation or shame. Staff engaged in avoidance behaviours by intentionally not discussing fatness in the presence of fat patients, pretending the patient was not fat during care situations or used indirect communication when it was necessary to discuss specific 'fat' management issues with colleagues. Likewise, when a person's face was threatened, corrective actions were taken where staff pretended not to notice the indiscretion which allowed the fat patient or fat staff member to maintain poise (Goffman, 1967). The purpose of all of these actions and behaviours by staff was to maintain the face of self, and patient; as to maintain one's 'face' was to 'fit in' (Lerner, 1996).

Avoidant face-work action assisted in preventing a threat to 'face' occurring and included avoidance of conversations related to the patient's fatness either during staff handovers in the patient's presence or directly with the fat patient. These avoidance face-work acts align themselves with the principles of Goffman's (1967) avoidance processes. However, unlike Goffman's (1967) avoidance strategies, which were instigated by the individual with the potential threat to 'face' (the fat patient), 
these strategies were employed by the other person (staff member) to prevent the threat to the 'face' of the patient.

These types of avoidance behaviours shared similarities with those exhibited during interactions between healthcare professionals and dying patients (Butow et al., 2008; Tay, Hegney, \& Ang, 2011; Wilkinson, 1991). In the above studies, healthcare professionals used blocking or inhibiting behaviours, such as making normalising or stereotyped comments, changing the topic, ignoring or being selective about addressing patient cues and jollying along the patient. These actions allowed staff to be able to distance themselves from uncomfortable and emotionally loaded areas of conversation by preventing patients from discussing their problems, worries and emotional concerns. The reasons for these behaviours were often related to personal fears of dying (Wilkinson, 1991), being overly task-orientated (Tay et al., 2011; Wilkinson, 1991), having negative attitudes (Tay et al., 2011), and providing care that was perfunctionary (Tay et al., 2011). In contrast, staff caring for fat patients in ICU used avoidance or blocking behaviours due to the fear of upsetting or offending the patient, or unintentionally revealing personal prejudicial attitudes.

The use of secrets codes, such as euphemisms, indirect speech, and non-verbal gestures were used as face-saving acts during staff-to-staff conversations about a patient's fatness when in the 'co-presence' of fat patients. Additionally, they were used as a way of expressing humour between ICU staff in backstage regions. These types of communication tactics have been identified in other health studies where sensitive topics of conversation were broached (Brown \& Thompson, 2007; Costello, 2001; Main, 2002; Zuzelo \& Seminara, 2007).

Many of the communication tactics used by ICU staff resembled behaviours exhibited by primary care nurses when specifically discussing weight management with clients (Brown \& Thompson, 2007). These strategies included softening the terms used to describe fatness, generally avoiding the term 'obesity' due to its negative connotation, avoiding directness by talking around the related issues, avoiding sterotypes and/or overly simplistic explanations (Brown \& Thompson, 2007). Additionally, fat nurses used self-disclosure as a way of lessening the awkwardness of 
conversations. This technique was orientated to demonstrating personal understanding and rapport, however, this equally raised issues of good role-modeling during consultations (Brown \& Thompson, 2007), as each communication tactic was aimed at maintaining a rapport which was patient focused. Likewise, Zuzelo and Seminara (2006) reported that nurses carefully monitored their facial expressions and body language during care situations so as to present a professional demeanour of being respectful, cordial and non-prejudice. Despite the different contexts of whether fatness was central or peripheral to the conversation, communication tactics were similar across studies, where the main emphasis was on preventing unnecessary loss of 'face' for the patient which would result in embarrassment or hurt.

Euphemisms within nursing and medicine have been used routinely as polite ways of referring to taboo subjects (Costello, 2001; Main, 2002), stigmatising conditions (Collier, 2010), and discussing altered bodily functions (Wald, 2007). In most cases, euphemisms have been used to conceal uncomfortable feelings felt by staff (Main, 2002), to disguise non-disclosed patient information (Costello, 2001), and prevent patient and staff feelings of discomfort or embarassment (Wald, 2007). In both ICU and Costello's (2001) work, euphemisms were used to communicate between healthcare teams in the presence of patients however, their use within conversations served different purposes. Costello (2001) identified that euphemisms allowed staff to continue having conversations regarding non-disclosed information whereas, ICU staff used euphemisms as a way to save 'face' by lessening the harshness of the communication to which the patient could hear.

Likewise, indirect speech, an example of a secret code, was used during conversations between ICU staff in the presence of fat patients as a way to share information that was only intended for the other staff member to understand. Indirect speech has been defined as a type of communication which allowed a speaker to say something that he does not literally mean but the hearer interprets it as it was intended (Pinker, 2009). The purpose of indirect speech was to prevent embarrassment, avoid awkwardness, save 'face' and reduce tension (Pinker, 2009). Although the indirect speech used by ICU staff adhered to the definition described by Pinker (2009), it differed from much of the literature on its use as a form of face-saving. More 
commonly indirect speech has occurred as a face-saving act in situations where a request was made of another (Lee \& Pinker, 2010; Pinker, 2009). The indirectness of the request allowed for the 'face' of both individuals (the speaker and the hearer) to be maintained should the request be refused.

Politeness theory has been used to explain the social motives that underpinned indirect speech and face-saving acts during social interaction (Brown \& Levinson, 1987). In Brown and Levinson's (1987) politeness theory any requests made of another was considered a threat to the 'negative face' of the hearer due to the potential to limit their automony. The use of politeness, which was considered the most commonest form of indirect speech, assisted speakers to soften the request and potential threat to the 'negative face' of the hearer (Brown \& Levinson, 1987; Pinker, 2009).

Even though making requests of another was not the intention of the indirect speech act by ICU staff, there were some similarities shared with the work of Lee and Pinker (2010) and Pinker (2009) which can be used to understand why this type of communication was used in the 'co-presence' of fat patients. Firstly, in many cases the indirect speech act delivered a negative message but the literal content was positive or neutral (Lee \& Pinker, 2010). This was observed regularly during staff handovers, where staff phrased the patient's excess body fat in positive endearing ways as a means to disguise from the patient the negative message delivered during the sharing of information between staff.

Secondly, when uncertain of whether the hearer, or other ICU staff member, was co-operative or antagonistic, the speaker could plausibly deny the intended message (Lee \& Pinker, 2010). A co-operative hearer was considered someone who was agreeable with the intended meaning, whereas an antagonistic hearer was someone who was offended by the intended message and acted against the speaker (Lee \& Pinker, 2010; Pinker, 2009). In the latter situation, the speaker could plausibly deny any knowledge of the intended message, claiming that the direct literal meaning was all that was meant. For example, a staff member might not be sure if their colleague shared the same private 'face' perceptions of fatness as them, and if not, the 
negative intended message could be plausibly denied if offense was taken. This strategy of communicating with each other allowed for the 'face' of themselves and others present to be saved.

Thirdly, indirect speech specifically allowed for the private and professional 'face' to be simultaneously presented during social interactions in the 'co-presence' of fat patients. While the literal content of the words spoken presented the professional 'face' of the empathic and kind professional; the intended message embedded in the spoken words presented private 'face' feelings of judgement, humour, and disapproval. In using indirect speech, staff were able to present two patient stories simultanously: the official 'frontstage' and the unofficial 'backstage' version of patient events (Goffman, 1959, 1961b).

The official version was the literal meaning meant for the patient while the unofficial version was the intended message meant for the other staff member (Goffman, 1959, 1961b; Pinker, 2009). During bedside handovers, the two staff members had no doubt about the intentions of the indirect speech because they knew the "back-story" (Pinker, 2009, p.80). In this case, the back story was the known private 'face' feelings and secret codes observed in each others' behaviours. However, the fat patient observing the interaction from a distance, lacked this secret information, and had only the actual words to go on. Nevertheless, some fat patients were capable of deciphering the implicature ${ }^{48}$, although their level of certainty was considerably less than that of the ICU staff (Pinker, 2009). This meant that ICU staff had the ability to plausibly deny the implied message if challenged or offense was taken thus, saving the 'face' of all involved in the encounter.

Face-saving acts exhibited characteristics of the phenomenon defined as mutual pretense by Glaser and Strauss (1965) in their work on awareness contexts of dying in hospital. An awareness context referred to what each person in the situation knew about the identity of the other person and the perceptions of what that person knew about him (Glaser \& Strauss, 1964, 1965). Glaser and Strauss (1965)

\footnotetext{
${ }^{48}$ Implicature is speech that involves two meanings: the literal content known as the sentence meaning, and the intended message known as the speaker meaning (Pinker 2009).
} 
constructed four awareness contexts which explained the management of knowledge, in their case dying, between two people or groups of people. The different awareness contexts were: closed, suspicion, mutual pretense, and open (Glaser \& Strauss, 1965).

Closed awareness context referred to situations when staff knew the patient was dying but this information was withheld from the patient who was unaware of the situation. Suspicion awareness context occurred when the dying patient began to suspect they were going to die. The concept of mutual pretense was used to explain situations where both hospital staff and dying patients knew the patient was dying but neither acknowledged this fact during interactions with each other. Open awareness referred to the open acknowledgement that the patient was dying by both staff and dying patient (Glaser \& Strauss, 1965).

Mutual pretense awareness was observed to be present in many of the interactions where the awareness of fat stigma influenced and affected the interactions between staff and fat patients. In ICU, mutual pretense awareness was a modification of open awareness, because both staff and fat patient were fully aware of the patient's fatness but pretended not to be. This awareness context occurred in situations where the fat patient concurred with the pretense of ICU staff, who were pretending the patient was not fat. Neither persons' acknowledged or mentioned anything to do with fatness. This way of interacting with each other was mutually beneficial for saving the 'face' of both staff and patient by helping to alleviate embarrassment or shame in certain care situations. For example, when a patient did not fit easily into care equipment neither staff or patient acknowledged this fact and both continued on as if nothing was amiss. This act of not noticing the threat to the fat patient's 'face', Goffman (1967) referred to as "tactful blindness" (p.18). However, on occasion, the staff pretense that the patient was not fat was challenged by the fat patient through direct conversations about their fatness in an attempt to achieve an open awareness context. In this situation, ICU staff encountered a threat to 'face', as their 'line' of pretense was challenged.

Unlike Glaser and Strauss's (1965) awareness contexts where the physical deterioration of dying patients moved knowledge and conversations into the final 
construct of open awareness, it was found that open awareness was not an inevitable endpoint of awareness contexts as ICU staff and fat patients could maintain the status quo of mutual pretense indefinitely. In ICU, staff preferred to maintain the pretense, despite attempts made by fat patients to move to open awareness. The reluctance by staff to move to a context of open awareness, if not initiated by the patient, was related to the uncertainty of how the fat patient would respond to direct acknowledgement in terms of 'percieved' or 'felt' stigma. Thus, there was a real tension between the association of fat stigma and open awareness which produced a social awkwardness within care situations.

What differentiates this study from the original understandings of knowledge management in the awareness context of dying (Glaser \& Strauss, 1965) was visibility and the type of knowledge that required management. The visibility of fatness meant that there was no closed or suspicion awareness context as everyone could see the patient was fat and the patient knew he/she was fat. Secondly, the management of knowledge was not about whether someone was aware they were fat but instead about the management of fat stigma during interactions, specifically 'enacted' stigma by ICU staff, or 'percieved' stigma felt by fat patients.

This study shared some similarities with Hyde's (1998) work where the stigma of sexual immorality of non-marital childbearing influenced the awareness context and management of knowledge during interactions. Equally, neither closed or suspicion awareness context were considered to be related to the encounters of pregnant women in the Republic of Ireland. The women in Hyde's (1998) study believed that even before the pregnancy was visible the other person knew about it due to conversations with close friends and family, therefore closed and suspicion awareness contexts did not feature in the narratives of the women in the study. However, it could be possible for these constructs of closed and suspicious awareness contexts to be applicable to knowledge management of pregnancy if the pregnant women has not told anyone. In this instance, the management of knowledge was very much more in fitting with Glaser and Strauss's (1965) trajectory of transitioning from a mutual pretense to open awareness context. 
Although, mutual pretense awareness was used by ICU staff and fat patients as a means to minimise embarrassment or shame, by maintaining 'face', it equally sustained the sense of apprehension and tension during interactions. In the studies by Hyde (1998) and Wittenberg-Lyles, Goldsmith, and Ragan (2011), a move to open awareness allowed for the easing of social tensions, and moderated stress levels, particularly in relation to the apprehension associated with the pretense. Open awareness in the dying context was considered to be beneficial as the the patient had the opportunity to plan for end-of-life. Similarly, for pregnant women the acknowlegement of the potentially stigmatising aspect of their identity through open awareness allowed for the normalising of the event of being pregnant.

Open awareness, Glaser and Strauss (1965) suggested, did not completely remove the complexity of the encounter as the details around the event, such as death still needed to be carefully managed and negotiated between all involved. Thus, in the case of stigma related awareness contexts, some topics of conversation were intentionally avoided which could socially discredit the person (Hyde, 1998). This fear of causing offense to fat patients appeared to impede the move to open awareness in encounters between staff and fat patients.

\subsection{Chapter summary}

Drawing on the literature, this chapter discussed the key findings of fat patients as 'misfits' and how staff managed their private perceptions of fatness during care situations through the use of emotional labour, behavioural regions, and facework. The study highlighted the care challenges related to the 'fit' between the patient and the environment in terms of size and design, and the social tension that was present that related to the 'fit' between the fat person's 'percieved' and 'expected' social behaviours in terms of healthy lifestyle decisions. It was shown how staff concealed, modified and regulated their feelings and emotions regarding fatness through the presentation of two different 'faces', the professional and private 'face'. The professional caring 'face' was presented when staff were in the 'co-presence' of fat patients and fat staff, whereas the private comtemptous 'face' was presented during interactions with other normal weight staff. In the concluding chapter I explore the new contributions to knowledge and methodology this study has made. I further 
critique the study limitations, discuss the implications that this new knowledge has for healthcare practice, and make recommendations for future research. 


\section{CHAPTER SEVEN: CONCLUSION}

\subsection{Introduction}

This primary research into how fat patients were cared for during a period of critical illness has demonstrated that interactions taking place during care were physically and socially challenging for staff. The research brought to the fore the previously unrecognised social awkwardness surrounding interactions and the emotional labour experienced by staff when caring for the fat patient population. The research demonstrated important new ways of understanding how social interactions and aspects of care, involving this socially stigmatised population of patients, were managed by private and professional 'face' activity of doctors and nurses. Private and professional 'face' activity is an important concept to understanding why direct discrimination, a component of fat stigma, was not present during the care of fat patients within the intensive care setting. The expression of prejudicial attitudes were confined to private 'face' activity and therefore concealed from the patient. Thus, direct acts of discrimination were not observed as staff adhered to their professional 'feeling' rules of their codes of professional conduct. The findings from the observations of doctors and nurses challenge previous assumptions in the literature by establishing that prejudicial attitudes of staff did not transpire into discriminating behaviours or care practices within the ICU setting.

\subsection{Contribution to knowledge}

The observations of ICU staff make an important contribution to nursing and healthcare knowledge by providing new perspectives in understanding the specific care challenges and needs of the critically ill fat patient, and how staff navigated, both personally and professionally, the social terrain of stigma when engaging in aspects of patient care. This study advances important theoretical knowledge of how emotional labour, behavioural regions, and face-work supported social interactions within the context of ICU practice, and identified social awkwardness as an emergent dimension of care that specifically related to the 'social novelty' of fatness.

Fat patients were misfits in the space of the ICU and posed significant clinical challenges for the ICU team caring for them. Although, the literature has previously 
described the physical and medical challenges of ICU practices, ICU staff in this study, used specific knowledge of shape, size, and type of body to inform the management of physical and medical care needs. ICU practices were primarily not informed by the patient's weight or BMI and consequently had very little informative meaning for staff when conducting care. More importantly, shape and size were the key determining factors of direct patient care issues and the focus of individual patient management strategies. Staff used this knowledge to adapt care practices to those which were more in fitting with the needs of specific fat body morphologies. This original contribution that fat patients were misfits in the ICU has implications, not only for how ICU services are developed, but also for other healthcare settings.

The social challenges to caring for the fat patient related to the negative personal feelings and emotions held by ICU staff regarding fatness. The negative feelings and emotions of staff needed to be managed during social interactions with fat patients and therefore separated into different spaces within the ICU through the construction of private and professional 'faces'. Although, the nursing literature has previously described the place of emotional labour in the construction of different 'faces', its use in this study served a different and very specific purpose. Emotional labour was used in the management of prejudicial attitudes during interpersonal social interactions.

In alignment with Hochschild's conceptualisation of emotional labour, ICU staff engaged in emotional labour as a way to separate out conflicting values and beliefs of the personal and professional 'self' in regards to fat patients. This conflict was primarily caused by the inherent dislike of fatness and what that represented in society. The emotional burden encountered by staff occurred from the efforts to reconcile their conflicting beliefs and values, and from working hard on suppressing and concealing private emotions when engaged in physically exhausting work of caring for the fat body. Emotional labour strategies were specifically focused on managing private emotions of dislike and judgement during social interactions so that care provision was observably indistinguishable from that of other patients. This use of emotional labour makes a substantial contribution to advancing Hochchild's original conceptualisation by expanding its understanding of the interpersonal 
management of emotions during interactions with socially stigmatised patient populations, and within the ICU setting.

Social awkwardness for ICU staff was a significant issue in the management of fat patients. Managing socially awkward moments has been identified as an emergent dimension of caring for fat patients. Social awkwardness added to the burden of care, in terms of the emotional labour and specific face-work required to maintain positive social interactions. Social awkwardness existed prior to any interaction, upon initiating social interactions, and during ongoing encounters with fat patients. The 'social novelty' of fatness in conjunction with the pre-existing social tensions of fat stigma were identified as precursors to many socially awkward interactions. Within the ICU, awkward situations occurred from the knowledge that fat patients did not fit into the space of the ICU, uncertainty of how to acknowledge the person's fatness or engage in direct conversations, or when other staff drew attention to the person's differences. This awkwardness was significantly amplified when engaging in physical aspects of care or discussing the patient's fatness in their presence. The research has brought to the fore the previously unrecognised anxiety and social awkwardness experienced by staff that surrounds interactions when caring for fat patients. It extends the understandings of the developing models of social awkwardness through its application to healthcare settings, and stigmatised health conditions by describing the situations in which awkwardness occurred and how it was managed in the context of fatness and care practices.

ICU staff placed considerable importance on the use of 'co-presence' rather than the physical location within ICU, to inform their behaviours. The unique setting of the ICU meant that there were specific physical and awareness barriers that frequently disrupted the 'co-presence' between different groups of people such a staff, patients, and visitors. These barriers included visiting times, the physical layout of the unit, and the often unconscious state of the patient. Each of these barriers impaired the ability to sense the presence of other people thus, disrupting the necessary conditions for 'co-presence'. Therefore, within the clinical zones of the ICU traditionally considered frontstage behavioural regions, there was very often an absence of 'copresence' between different groups of people. This meant that behavioural regions 
could co-exist in the same physical spaces of the ICU. This important new and original finding that 'co-presence' determined the behavioural regions and 'face' presented, has implications for understanding the social context of interactions and expressions of emotions in other healthcare settings.

How healthcare professionals engaged in face-work to maintain positive social interactions has been previously described in the healthcare literature. However, this study established that the specific face-saving acts of indirect speech and euphemisms used frequently during staff conversations, allowed for the private 'face' to lie behind the professional 'face' presented in the 'co-presence' of fat patients. In these encounters, the literal content of the spoken words presented the professional 'face'; whilst the back story to the intended message embedded in the words revealed the private 'face' world. Staff were therefore able to present two stories simultaneously to two audiences: the official frontstage for the patient and unofficial backstage version of events for other staff. The presentation of two simultaneous stories within the same physical space challenges the understanding of Goffman's 'stages' as being two separate regions. This work demonstrates how the front and back regions were not separate identifiable geographical spaces per se, but one space where the conditions within that space shifted the front and back stage.

\subsection{Contribution to methodology and method}

This study contributes to ethnographic methodology and methods of data collection by reconceptualising the insider-outsider position. Previous conceptualisations of the insider-outsider position have described this from a static perspective; the researcher either adopts an insider or an outsider position within the field. From this 'static' position, the researcher reflexively describes the reasons for adopting this position and the events within the field that challenged the field position chosen.

The insider-outsider position adopted in this study was not static, but dynamic and constantly changing as I responded to professional, ethical and research situations of the field. In making such responses, I found myself moving in and out of the central functioning of my social group, and across nurse and researcher roles. The 
competing tension between my duty of care and adhering to the principles of research constantly placed me to different spaces within the field. Therefore, the space that I occupied became situational to the needs of the field and were determined by both the actions instigated by myself and by the perceptions and demands of the study participants.

This emergent dynamic-situational model requires the researcher to consciously consider what the impact of his/her involvement in a situation would be on the study participants and the research process. In doing so, the nurse researcher consciously moves across situations occupying different spaces within the field. This work demonstrated that the insider-outsider position was not a static position by offering a dynamic and emergent model where the insider-outsider position becomes situational to the requirements of the field. This methodological contribution not only has implications for how nurse researchers engage in observational studies of clinical practice but also for other disciplines in which the researcher has a dual identity.

\subsection{Methodological critique of the study}

The strength of this study was inherent in the choice of focused ethnography as the appropriate research approach to elicit specific knowledge of experiences of ICU staff in caring for fat patients. Additionally, the use of observation as the primary mode of cognition allowed for the identified knowledge gap between self-reported attitudes and actual behaviours to be addressed. This approach of combining observations of staff-patient interactions with self-reported attitudes and beliefs offers new insights into the emerging evidence on how attitudes and actual behaviours are related during the care of critically ill fat patients.

Both a strength and a limitation of this study was the intention to only understand the 'situated' experiences of the ICU staff as they engaging in the care of critically ill fat patients. The strength of this approach meant that issues pertinent to the staff in the delivery of ICU services to fat patients became the primary focus of the research. The limitation was that only one perspective of the social interaction during care was recorded. If I had conducted interviews with patients about their care 
and ICU admission it may have developed a greater understanding of the clinical issues and how patient's managed their emotions during care situations.

A further limitation of this study was the use of one study site. Other tertiary ICU's within New Zealand may have different resources, and ways of delivering care to fat patients which would not permit the generalisability of findings to other ICU services. This issue has been addressed by providing enough contextual information regarding the study site and methodology to allow others to determine the transferability of findings to their own situations. By using one study site, this ethnographic research offers depth rather than breadth of understanding in caring for critically ill fat patients.

\subsection{Implications for healthcare practice}

Implications for healthcare practice arising from this study that attend to the specific needs of fat patients during hospital admissions, and support staff in managing their emotions, needs to be acknowledged and further developed. ICU practices have been developed that clinically assess the fat body, beyond BMI and weight, to support specific individual patient intervention and management needs, as highlighted in this study. Whilst the use of BMI remains an important measure of fatness to inform national level policies that address fatness in terms of predicting population based health risks, health promotion strategies, and specific resourcing and funding, it has very limited use in developing services fit for individual patient care needs. To support the development and implementation of bariatric care services locally, care frameworks need to be underpinned by more suitable and meaningful body measurements which more appropriately assess the fit between the patient and supporting environment. It is suggested, from this study, that developing bariatric care pathways that meet the individual needs of fat patients require:

- Greater collaboration and development of partnerships between the healthcare industry and commercial agencies to address the misfit in size and design of available resources such as specialised equipment, gowns, and medical intervention equipment; 
- Development of assessment tools and admission to discharge pathways that are based on shape, size and types of bodies;

- Development of local policies and procedures to support tender, and acquisition of resources;

- Provision of education and training programmes that specifically address care issues and increase knowledge of population specific anatomy and physiology, care challenges and management, clinical assessment, and best practice, such as positioning, equipment selection and manual handling, that support local service initiatives.

The management of personal emotions for staff during care was a significant issue raised in this study. Healthcare organisations need to acknowledge and be responsive to the burden of emotional labour that exists in providing care to fat patients and recognise that the impact of emotional stressors are not limited to situations of death and dying. By acknowledging the emotions involved in care, and addressing the emotional component of managing this patient population, any outcome of long-term exposure to the burden of emotional labour can be reduced. Developing appropriate support structures to better equip staff to manage the emotional labour of caring for fat patients require:

- Senior staff to take lead roles in being proactive in monitoring, assessing and managing the emotional labour and social tensions that are present when caring for fat patients within the clinical environment;

- Provision of private safe spaces for staff to be able to unload their emotions and feelings, between care situations that require them to maintain a professional caring 'face';

- Provision of support systems for staff such as forums, professional development, and debriefing sessions that allow for opportunities to talk openly about emotions and reactions to caring for fat patients, seek 
strategies for managing the personal effects of the emotional burden, and develop skills in addressing the social awkwardness of caring for fat patients.

Whilst there is an immediate need to provide safe spaces for staff to attend to the emotional burden of caring for fat patients, there is still a requirement to address the existing stigma attached to fat patients within the healthcare setting. Health care professionals need to consciously challenge current perspectives and practices, both personally and professionally, and become advocates for fat patients so that they can influence decisions within political, economic and professional spheres that impact on health care services for fat patients. Developing professional advocacy and leadership roles to address care and stigma issues requires:

- Health care professionals to acknowledge personal responsibility to engage in professional development activities, such as reflexivity, as a means to confront/question their personal perspectives on fatness and caring for fat patients

- The development and provision of education and training programmes that specifically address issues of fat stigma and focus on stigma reduction interventions

- The provision of professional development opportunities for staff to develop key leadership and advocacy skills that can support and promotes social justice for fat patient's in terms of health care rights and enhance policy initiatives that focus on the availability, safety and quality of care for fat patients.

\subsection{Recommendations for future research}

The above implications for healthcare practice provide potential areas for research in the field of fatness and disease related stigma. There are a number of recommendations specific to potential research that stem from this study. Further qualitative research is required on the experiences of healthcare professionals caring for fat patients within other in-patient hospital settings. This would provide additional 
insights and comparisons beyond the ICU setting and allow for a broader understanding of the issues, challenges and management strategies used during care.

Like healthcare staff, fat patients have their own 'situated' knowledge and lived experiences of engaging with and being cared for by healthcare staff. Further research is needed to explore these experiences and perspectives of care within the intensive care, and wider hospital setting. Additionally, a more focused research approach exploring how fat patients manage their relationships, and social interactions with healthcare staff is warranted. This area of research is already under development through a qualitative descriptive study, which explores the fat patients' experiences of their hospital stay from admission to discharge, in which I am the lead investigator.

Further research is also needed to examine the knowledge gap between attitudes and actual behaviours of healthcare staff caring for fat patients. For example, a mixed methods approach using psychometric measurements of attitudes, beliefs and intended behaviours in conjunction with observations of actual behaviours during care situations would offer insights into the congruence of staff attitudes and behaviours. More work is needed to develop the psychometric measurements currently used to assess attitudes, beliefs, and intended behaviours of staff caring for fat patients. In particular, consideration should be given to incorporating the components of emotional labour into the intended behaviour measurements, which would be more reflective of care processes.

A potential area of research is to explore whether the types of emotion management used by healthcare staff in the interactions with fat patients were disease specific or applicable to other socially stigmatised health conditions. An ethnographic approach, similar to one taken in this study, would allow for the examination of the social interactions and management of care within in the natural environment, and enable comparisons across studies to be made. In light of the study findings, more research is needed on developing robust physical and medical assessments of fat patients that inform care pathways, organisational policies, and resource needs from hospital admission to discharge. Research in this area needs to adopt a multi- 
disciplinary team approach and include clinical trials, feasibility studies, and implementation studies. Furthermore, the New Zealand cultural value and belief systems regarding fatness and the cultural influence on the context of social interactions may not reflect other cultures. Therefore, international research on this topic is warranted.

\subsection{Conclusion}

Fatness has been cited as the last socially accepted form of discrimination in society; the pervasiveness of fat stigma is so strong that no one is immune; and logically therefore weight bias leads to discrimination. These frequently cited statements within the literature has lead to assumptions that all individuals, including healthcare professionals, hold strong fat stigma and by doing so discriminate against fat patients during care situations. The findings of this study challenge this position by establishing how the prejudicial attitudes and beliefs of healthcare staff were modified and regulated through the use of professional and private 'face' activity. Prejudice was confined to the private 'face' and therefore, hidden from the fat patient. During social interactions and when providing care, direct acts of discrimination were not observed, as staff adhered to their 'feeling' rules of their codes of professional conduct. Staff were pleasant, empathetic, and kind in their interactions with fat patients. Conscious of the social awkwardness that existed, as a result of not 'fitting' into the space of the ICU and the social stigma of being fat, staff engaged in specific types of face-work to address potential issues of embarrassment, humiliation and shame. This research has demonstrated new ways of understanding how social interaction and aspects of care, that involved socially stigmatised fat patients, were managed to deliver care that was observably indistinguishable from other patients. 


\section{REFERENCES}

Ääri, R. L., Tarja, S., \& Helena, L. K. (2008). Competence in intensive and critical care nursing: A literature review. Intensive and critical care nursing, 24(2), 78-89. doi: http://dx.doi.org/10.1016/j.iccn.2007.11.006

Abbey, S., Charbonneau, M., Tranulis, C., Dabby, L., Gautam, M., \& Pare, M. (2011). Stigma and discrimination. Position paper. The Canadian Journal of Psychiatry, 56(10), 1-9.

Aberson, C. L., Healy, M., \& Romero, V. (2000). Ingroup bias and self-esteem: A metaanalysis. Personality and Social Psychology Review, 4(2), 157-173. doi: 10.1207/s15327957pspr0402_04

Adams, C. H., Smith, N. J., Wilbur, D. C., \& Grady, K. E. (1993). The relationship of obesity to the frequency of pelvic examinations. Women and Health, 20(2), 45-57. doi: 10.1300/J013v20n02_04

Adams, J. P., \& Murphy, P. G. (2000). Obesity in anaesthesia and intensive care. British Journal of Anaesthesia, 85(1), 91-108. doi: 10.1093/bja/85.1.91

Allen, D. (2004). Ethnomethodological insights into insider-outsider relationships in nursing ethnographies of healthcare settings. Nursing Inquiry, 11(1), 14-24.

Allison, D. B., Downey, M., Atkinson, R. L., Billington, C. J., Bray, G. A., Eckel, R. H., . . . Tremblay, A. (2008). Obesity as a disease: A white paper on evidence and arguments commissioned by the council of the obesity society. Obesity, 16(6), 1161-1177. doi: http://dx.doi.org/10.1038/oby.2008.231

Allport, G. W. (1954). The nature of prejudice. California: Addison-Wesley.

Alpert, M. A., Fraley, M. A., Birchem, J. A., \& Senkottaiyan, N. (2005). Management of obesity cardiomyopathy. Expert Review of Cardiovascular Therapy, 3(2), 225-230. doi: http://dx.doi.org/10.1586/14779072.3.2.225

Amy, N. K., Aalborg, A., Lyons, P., \& Keranen, L. (2006). Barriers to routine gynecological cancer screening for white and African-American obese women. International Journal of Obesity, 30(1), 147-155. doi: http://dx.doi.org/10.1038/sj.ijo.0803105

Anderson, E. (2002). The ideologically driven critique. American Journal of Sociology, 107(6), 1533-1550. doi: 10.1086/342772

Angaramo, G. G. (2010). Chest tube placement and care. In R. S. Irwin \& J. M. Rippe (Eds.), Manual of intensive care medicine (5th ed., pp. 47-53). Baltimore: Lippincott Williams and Wilkins.

Araghi, A., Bander, J., \& Guzman, J. (2006). Arterial blood pressure monitoring in overweight critically ill patients: Invasive or noninvasive? Critical Care, 10(2), R64.

Arber, A. (2006). Reflexivity: A challenge for the research practitioner? Journal of Nursing in Research, 11(2), 147-157.

Aronne, L. J., Nelinson, D. S., \& Lillo, J. L. (2009). Obesity as a disease state: A new paradigm for diagnosis and treatment. Clinical Cornerstone, 9(4), 9-29. doi: http://dx.doi.org/10.1016/S1098-3597(09)80002-1

Ashforth, B. E., \& Humphrey, R. H. (1993). Emotional labor in service roles: The influence of identity. The Academy of Management Review, 18(1), 88-115. doi: 10.2307/258824

Ashwell, M., \& Gibson, S. (2009). Waist to height ratio is a simple and effective obesity screening tool for cardiovascular risk factors: Analysis of data from the British national diet and nutrition survey of adults aged 19-64 years. Obesity Facts, 2(2), 97103. 
Asselin, M. E. (2003). Insider research: Issues to consider when doing qualitative research in your own setting. Journal for Nurses in Staff Development, 19(2), 99-103.

Atkinson, P., \& Silverman, D. (1997). Kundera's immortality: The interview society and the invention of self. Qualitative Enquiry, 3(3), 304-325.

Australian and New Zealand Intensive Care Society. (2013). Centre for Outcome and Resource Evaluation (CORE) Annual Report 2011-2012. Melbourne: ANZICS.

Bagley, C., Conklin, D., Isherwood, R., Pechiulis, D., \& Watson, L. (1989). Attitudes of nurses towards obesity and obese patients. Perceptual and Motor Skills, 68, 954.

Bahammam, A. S., \& Al-Jawder, S. E. (2012). Managing acute respiratory decompensation in the morbidly obese. Respirology, 17(5), 759-771. doi: 10.1111/j.14401843.2011.02099.x

Bajwa, S. J., Sehgal, V., \& Bajwa, S. K. (2012). Clinical and critical care concerns in severely ill obese patient. Indian Journal of Endocrinology and Metabolism, 16(5), 740-748.

Bargiela-Chiappini, F. (2003). Face and politeness: New (insights) for old (concepts). Journal of Pragmatics, 35(10-11), 1453-1469. doi: http://dx.doi.org/10.1016/S03782166(02)00173-X

Barrett, R. A. (1991). Culture and conduct: An excursion in anthropology (2nd ed.). California: Wadsworth Publishing Company.

Bersten, A., \& Soni, R. (2009). Oh's intensive care manual (6 ed.). London: Elsevier.

Bolton. (2000). Who cares? Offering emotion work as a 'gift' in the nursing labour process. Journal of Advanced Nursing, 32(3), 580-586.

Bolton, S. C. (2001). Changing faces: Nurses as emotional jugglers. Sociology of Health and Illness, 23(1), 85-100. doi: 10.1111/1467-9566.00242

Bone, D. (2002). Dilemmas of emotion work in nursing under market-driven health care. The International Journal of Public Sector Management, 15(2), 140-150.

Bonner, A., \& Tolhurst, G. (2002). Insider-outsider perspectives of participant observation. Nurse Researcher, 9(4), 7-19.

Bowling, A. (1997). Research methods in health. Buckingham, UK: Open University Press.

Bradbury-Jones, C. (2007). Enhancing rigour in qualitative health research: Exploring subjectivity through Peshkin's I's. Journal of Advanced Nursing, 59, 290-298. doi: 10.1111/j.1365-2648.2007.04306.x

Branaman, A. (1997). Goffman's social theory. In C. Lemert \& A. Branaman (Eds.), The Goffman reader (pp. xlv-Ixxxii). Oxford: Blackwell.

Bromley, C., \& Given, L. (2011). Scottish health survey 2010 (Vol. 1). Scotland: Scottish Centre for Social Research.

Brown, I. (2006). Nurses' attitudes towards adult patients who are obese: Literature review. Journal of Advanced Nursing, 53(2), 221-232. doi: 10.1111/j.13652648.2006.03718.x

Brown, I., Stride, C., Psarou, A., Brewins, L., \& Thompson, J. (2007). Management of obesity in primary care: Nurses' practices, beliefs and attitudes. Journal of Advanced Nursing, 59(4), 329-341. doi: 10.1111/j.1365-2648.2007.04297.x

Brown, I., Thompson, J., Tod, A., \& Jones, G. (2006). Primary care support for tackling obesity: A qualitative study of the perceptions of obese patients. British Journal of General Practice, 56(530), 666-672.

Brown, I., \& Thompson, J. M. (2007). Primary care nurses' attitudes, beliefs and own body size in relation to obesity management. Journal of Advanced Nursing, 60(5), 535-543. doi: 10.1111/j.1365-2648.2007.04450.x

Brown, P., \& Levinson, S. C. (1987). Politeness: Some universals in language usage. Cambridge: Cambridge University Press. 
Brownell, K. D., \& Puhl, R. M. (2003). Stigma and discrimination in weight management and obesity. The Permanente, 7(3), 21-23.

Brownell, K. D., Puhl, R. M., Schwartz, M. B., \& Rudd, L. (2005). Weight bias: Nature, consequences and remedies. New York: Guildford Press.

Burgess, R. G. (1984). In the field: An introduction to fieldwork. London: Routledge.

Butow, P., Cockburn, J., Girgis, A., Bowman, D., Schofield, P., D'Este, C., . . Tattersall, M. (2008). Increasing oncologists' skills in eliciting and responding to emotional cues: Evaluation of a communication skills training programme. Psycho-Oncology, 17, 209218.

Buxton, B. K., \& Snethen, J. (2013). Obese women's perceptions and experiences of healthcare and primary care providers. Nursing Research, 62(4), 252-259. doi: 10.1097/NNR.0b013e318299a6ba

Cain, C. L. (2012). Integrating dark humor and compassion: Identities and presentations of self in the front and back regions of hospice. Journal of Contemporary Ethnography, 41(6), 668-694. doi: 10.1177/0891241612458122

Callahan, J. L., \& McCollum, E. E. (2002). Obscured variability: The distinction between emotion work and emotional labour. In N. M. Ashkansay, W. J. Zerbe \& C. E. J. Hartel (Eds.), Managing emotions in the workplace (pp. 219-231). New York: Armonk.

Carr, D., \& Friedman, M. A. (2005). Is obesity stigmatizing? Body weight, perceived discrimination, and psychological well-being in the United States. Journal of Health and Social Behavior, 46(3), 244-259. doi: 10.2307/4147670

Casey, D. (2004). Challenges of collecting data in the clinical setting. Nursing Times Research, 9(2), 131-141. doi: 10.1177/136140960400900208

Cerinus, M. (2001). The ethics of research. Nurse Researcher, 8(3), 72.

Clegg, J. W. (2012a). The importance of feeling awkward: A dialogical narrative phenomenology of socially awkward situations. Qualitative Research in Psychology, 9(3), 262-278. doi: 10.1080/14780887.2010.500357

Clegg, J. W. (2012b). Stranger situations: Examining a self-regulatory model of socially awkward encounters. Group Processes and Intergroup Relations, 15(6), 693-712. doi: $10.1177 / 1368430212441637$

Clifford, J. (1983). On ethnographic authority. Representations, 2 (Spring), 118-146.

Coffey, A. (1999). The ethnographic self: Fieldwork and the representation of identity. London: Sage Publications.

College of Intensive Care Medicine of Australia and New Zealand. (2011). Minimum standards for intensive care units. (pp. 1-18): College of Intensive Care Medicine of Australia and New Zealand.

Collier, R. (2010). Who you calling obese, Doc? Canadian Medical Association Journal, 182(11), 1161-1162. doi: 10.1503/cmaj.109-3308

Colwell, J. (2004). Principles of stoma management. In J. Colwell, M. T. Goldberg \& J. E. Carmel (Eds.), Fecal and urinary diversions: Management principles. St Louis: Elsevier Health Sciences.

Condell, S. L. (2008). Writing fieldnotes in an ethnographic study of peers - Collaborative experiences from the field. Journal of Research in Nursing, 13(4), 325-335. doi: $10.1177 / 1744987107087555$

Coombs, M. A. (2004). Power and conflict between doctors and nurses: Breaking through the inner circle in clinical care. London: Routledge.

Cooper, C. (2010). Fat studies: Mapping the field. Sociology Compass, 4(12), 1020-1034. doi: 10.1111/j.1751-9020.2010.00336.x 
Costello, J. (2001). Nursing older dying patients: Findings from an ethnographic study of death and dying in elderly care wards. Journal of Advanced Nursing, 35(1), 59-68. doi: 10.1046/j.1365-2648.2001.01822.x

Couchman, W., \& Dawson, J. (1995). Nursing and healthcare research. London, UK: Scutari.

Crandall, C. S. (1994). Prejudice against fat people: Ideology and self-interest. Journal of Personality and Social Psychology, 66(5), 882-894. doi: http://dx.doi.org/10.1037/0022-3514.66.5.882

Crandall, C. S., \& Biernat, M. (1990). The ideology of anti-fat attitudes. Journal of Applied Social Psychology, 20, 227-243.

Crandall, C. S., \& Martinez, R. (1996). Culture, ideology, and antifat attitudes. Personality and Social Psychology Bulletin, 22(11), 1165-1176.

Creel, E., \& Tillman, K. (2011). Stigmatization of overweight patients by nurses. The Qualitative Report, 16, 1330-1351.

Crocker, J., \& Major, B. (1989). Social stigma and self-esteem: The self-protective properties of stigma. Psychological Review, 96, 608-630.

Cross, H. (2006). Interventions to address the stigma associated with leprosy: A perspective on the issues. Psychology, Health and Medicine, 11(3), 367-373. doi: $10.1080 / 13548500600595384$

Cruz, E. V., \& Higginbotton, G. (2013). The use of focused ethnography in nursing research. Nurse Researcher, 20(4), 36-43.

Cudmore, H., \& Sondermeyer, J. (2007). Through the looking glass: Being a critical ethnographic researcher in a familiar nursing context. Nurse Researcher, 14(3), 25-35.

Culbertson, M., \& Smolen, D. (1999). Attitudes of RN students toward obese adult patients. Journal of Nursing Education, 38(2), 84-87.

Cupach, W. R., Metts, S., \& Hazleton, V. (1986). Coping with embarrassing predicaments: Remedial strategies and their perceived utility. Journal of Language and Social Psychology, 5(3), 181-200. doi: 10.1177/0261927x8600500302

Davidson, J. E., Kruse, M. W., Cox, D. H., \& Duncan, R. (2003). Critical care of the morbidly obese. Critical Care Nursing Quarterly, 26(2), 105-118.

Davies, S., Ellis, L., \& Laker, S. (2000). Promoting autonomy and independence for older people within nursing practice: An observational study. Journal of Clinical Nursing, $9(1), 127-136$.

Davis, J. M. (2000). Disability studies as ethnographic research and text: Research strategies and roles for promoting social change? Disability and Society, 15(2), 191-205.

Dawson, D. (2006). The art of nursing: A hidden science? Intensive and critical care nursing, 22, 313-314.

De Laine, M. (1997). Ethnography: Theory and applications in health research. Sydney: MacLennan and Petty.

Department of Health. (2011). Healthy lives, healthy people: A call to action on obesity in England. London, UK: HM Government.

Department of Health and Ageing. (2007). Healthy active Australia- Promoting healthy living. Canberra: Australian Goverment.

Deutscher, I. (1973). What we say/ what we do. Glenview: Scott Foresman.

Diefendorff, J. M., \& Gosserand, R. H. (2003). Understanding the emotional labor process: A control theory perspective. Journal of Organizational Behavior, 24(8), 945-959. doi: $10.2307 / 4093748$

Dion, K. L., \& Dion, K. K. (1987). Belief in a just world and physical attractiveness stereotyping. Journal of Personality and Social Psychology, 52, 775-780. 
Dovidio, J. F., Major, J. E., \& Crocker, J. (2000). Stigma: Introduction and overview. In T. F. Heatherton, R. E. Kleck, M. R. Hebl \& J. G. Hull (Eds.), The Social Psychology of Stigma (pp. 1-29). New York: Guildford Press.

Drake, D., McAuliffe, M., \& Edge, M. J. (2012). Post operative nursing care of patients after bariatric surgery. Perspectives: Recovery strategies from OR to home, 6(4), 1, 5-8.

Drury, C., \& Louis, M. (2002). Exploring the association between body weight, stigma of obesity, and health care avoidance. Journal of the American Academy of Nurse Practitioners, 14(12), 554.

Duncan, E., Schofield, G., Duncan, S., Kolt, G., \& Rush, E. (2004). Ethnicity and body fatness in New Zealanders. Journal of the New Zealand Medical Association, Vol 117 (1195), 1-9.

Duneier, M. (2002). What kind of combat sport is sociology? American Journal of Sociology, 107(6), 1551-1576. doi: 10.1086/342925

Eagley, A. H., \& Chaiken, S. (1993). The psychology of attitudes. Florida: Harcourt Brace.

Eipper, C. (1998). Anthropology and cultural studies: Difference, ethnography and theory. The Australian Journal of Anthropology, 9(3), 310-326.

Eiser, J. R. (1997). Attitudes and beliefs. In A. Baum, S. Newman, J. Weinman \& C. McManus (Eds.), Cambridge handbook of psychology, health and medicine (pp. 1-6). Cambridge: Cambridge University Press.

El-Solh, A. (2004). Clinical approach to the critically ill morbidly obese patient. American Journal Respiratory Critical Care Medicine, 169, 557 - 561.

El-Solh, A., \& Jaafar, W. (2007). A comparative study of the complications of surgical tracheostomy in morbidly obese critically ill patients. Critical Care, 11(1), R3.

Elliott, R., \& Wright, L. (1999). Verbal communication: What do critical care nurses say to their unconscious or sedated patients? Journal of Advanced Nursing, 29(6), 14121420. doi: 10.1046/j.1365-2648.1999.01028.x

Emerson, R. M., Fretz, R. I., \& Shaw, L. L. (1995). Writing ethnographic fieldnotes. Chicago: The University of Chicago Press.

Evans, A., Pereira, D., \& Parker, J. (2013). Categorising the nurse-researcher during fieldwork: 'One of us' or 'one of them'? Journal of Research in Nursing, 18(8), 707717. doi: $10.1177 / 1744987112451577$

Fabricatore, A. N., Wadden, T. A., \& Foster, G. D. (2005). Bias in health care settings. In K. D. Brownell, R. M. Puhl, M. B. Schwartz \& L. Rudd (Eds.), Weight bias: Nature, consequences, and remedies. (pp. 29-53). London: The Guildford Press.

Fetterman, D. M. (2010). Ethnography: Step by step (3rd ed. Vol. 17). London: Sage Publications.

Fine, G. A., \& Manning, P. (2000). Erving Goffman. In G. Ritzer (Ed.), The Blackwell companion to major social theorists (pp. 457-485). Oxford: Blackwell.

Firestone, W. A. (1993). Alternative arguments for generalising from the data as applied to qualitative research. Educational Researcher, 22, 16-23.

Flegal, K. M., Kit, B. K., Orpana, H., \& Graubard, B. I. (2013). Association of all-cause mortality with overweight and obesity using standard body mass index categories: A systematic review and meta-analysis. Journal of the American Medical Association, 309(1), 71-82. doi: 10.1001/jama.2012.113905

Fleischer, S., Berg, A., Zimmermann, M., Wüste, K., \& Behrens, J. (2009). Nurse-patient interaction and communication: A systematic literature review. Journal of Public Health, 17(5), 339-353.

Fong, R. L., Bertakis, K. D., \& Franks, P. (2006). Association between obesity and patient satisfaction. Obesity, 14(8), 1402-1411. doi: http://dx.doi.org/10.1038/oby.2006.159 
Fontaine, K. R., Faith, M. S., Allison, D. B., \& Cheskin, L. J. (1998). Body weight and health care among women in the general population. Archives of Family Medicine, 7(4), 381-384.

Fontaine, K. R., Redden, D. T., Wang, C., Westfall, A. O., \& Allison, D. B. (2003). Years of life lost due to obesity. Journal of the American Medical Association, 289(2), 187-193. doi: 10.1001/jama.289.2.187

Forhan, M., \& Salas, X. R. (2013). Inequities in healthcare: A review of bias and discrimination in obesity treatment. Canadian Journal of Diabetes, 37(3), 205-209. doi: http://dx.doi.org/10.1016/j.jcjd.2013.03.362

Forhan, M. A., Law, M. C., Vrkljan, B. H., \& Taylor, V. H. (2010). The experience of participation in everyday occupations for adults with obesity. Canadian Journal of Occupational Therapy, 77(4), 210-218. doi: 10.2182/cjot.2010.77.4.3

Forhan, M. A., Vrkljan, B., \& MacDermid, J. (2010). A systematic review of the quality of psychometric evidence supporting the use of an obesity-specific quality of life measure for use with persons who have class III obesity. Obesity Reviews, 11(3), 222228. doi: 10.1111/j.1467-789X.2009.00612.x

Forsey, M. G. (2010). Ethnography as participant listening. Ethnography, 11(4), 558-572. doi: $10.1177 / 1466138110372587$

Foster, G. D., Wadden, T. A., Makris, A. P., Davidson, D., Sanderson, R. S., Allison, D. B., \& Kessler, A. (2003). Primary care physicians' attitudes about obesity and its treatment. Obesity Research, 11(10), 1168-1177. doi: 10.1038/oby.2003.161

Friedman, K. E., Reichmann, S. K., Costanzo, P. R., Zelli, A., Ashmore, J. A., \& Musante, G. J. (2005). Weight stigmatization and ideological beliefs: Relation to psychological functioning in obese adults. Obesity Research, 13(5), 907-916. doi: 10.1038/oby.2005.105

Froggatt, K. (1995). Keeping the balance: Hospice work, death and emotions. (Doctor of Philosophy), South Bank University, London.

Froggatt, K. (1998). The place of metaphor and language in exploring nurses' emotional work. Journal of Advanced Nursing, 28(2), 332-338. doi: 10.1046/j.13652648.1998.00688.x

Froman, R. D., \& Owen, S. V. (2001). Measuring attitudes towards persons with AIDS: The AAS-G as an alternative form of ASS. Scholarly Inquiry for Nursing Practice, 15, 161-174.

Gallagher-Camden, S. (2006). Nursing care of the bariatric patient. Bariatric Nursing and Surgical Patient Care, 1(1), 21-30. doi: http://dx.doi.org/10.1089/bar.2006.1.21

Gallagher-Camden, S. (2009). Obesity: An emerging concern for patients and nurses. The online journal of nursing issues, 14.

Galuska, D. A., Will, J. C., Serdula, M. K., \& Ford, E. S. (1999). Are health care professionals advising obese patients to lose weight? Journal of the American Medical Association, 282(16), 1576-1578. doi: 10.1001/jama.282.16.1576

Gard, M., \& Wright, J. (2005). The obesity epidemic: Science, morality and ideology. Abingdon: Routlege.

Garland-Thomson, R. (2005). Feminist disability studies. Signs, 30(2), 1557-1587. doi: $10.1086 / 423352$

Garland-Thomson, R. (2011). Misfits: A feminist materialist disability concept. Hypatia, 26(3), 591-609. doi: 10.1111/j.1527-2001.2011.01206.x

Garner, C., \& Nicol, G. (1998). Comparison of male and female nurses' attitudes towards obesity. Perceptual and Motor Skills, 86, 1442. 
Geertz, C. (1973). The interpretation of culture: Selected essays by Clifford Geertz Retrieved from http://hdl.handle.net/2027/heb.01005.0001.001 $<$ http://hdl.handle.net.helicon.vuw.ac.nz/2027/heb.01005.0001.001>

Gerrish, K. (1997). Being a 'marginal native': Dilemmas of the participant observer. Nurse Researcher, 5(1), 25-34.

Gilbert, N., \& Mulkay, M. (1983). In search of the action. In N. Gilbert \& P. Abell (Eds.), Accounts and action. Aldershot: Gower.

Gillis, A., \& Jackson, W. (2002). Research for nurses: Methods and interpretation. Philadelphia: F.A Davis Company.

Glaser, B. G., \& Strauss, A. L. (1964). Awareness contexts and social interaction. American Sociological Review, 29(5), 669-679. doi: 10.2307/2091417

Glaser, B. G., \& Strauss, A. L. (1965). Awareness of dying. Chicago: Aldine.

Glaser, B. G., \& Strauss, A. L. (1967). The discovery of grounded theory: Strategies for qualitative research. Chicago: Aldine.

Gobo, G. (2008). Doing ethnography. London, England: SAGE Publications.

Goffman, E. (1952). On cooling the mark out: Some aspects of adaptation to failure. Psychiatry, 15(4), 451-463.

Goffman, E. (1959). Presentation of self in everyday life. New York: Doubleday.

Goffman, E. (1961a). Fun in games. In E. Goffman (Ed.), Encounters: Two studies in the sociology of interaction (pp. 15-81). Indianapolis: Bobbs-Merrill.

Goffman, E. (1961b). Role distance. In E. Goffman (Ed.), Encounters: Two studies in the sociology of interaction (pp. 83-152). Indianapolis: Bobbs-Merrill.

Goffman, E. (1963a). Behaviour in public places: Notes on the social organisation of gatherings. New York: Free Press.

Goffman, E. (1963b). Stigma: Notes on the management of spoiled identity. New York: Simon and Schuster.

Goffman, E. (1967). Interaction ritual: Essays on face-to-face behaviour. New York: Anchor Books.

Goffman, E. (1969). Strategic interaction. Philadelphia: University of Pennsylvania Press.

Gold, R. (1958). Roles in sociological field observation. Social Forces, 36(3), 217-223.

Goldberg, A. E., \& Smith, J. Z. (2011). Stigma, social context, and mental health: Lesbian and gay couples across the transition to adoptive parenthood. Journal of Counseling Psychology, 58(1), 139-150.

Gray, B. (2009). The emotional labour of nursing- Defining and managing emotions in nursing work. Nurse Education Today, 29(2), 168-175. doi: http://dx.doi.org/10.1016/j.nedt.2008.08.003

Gray, B., \& Smith, P. (2009). Emotional labour and the clinical settings of nursing care: The perspectives of nurses in East London. Nurse Education in Practice, 9(4), 253-261. doi: http://dx.doi.org/10.1016/j.nepr.2008.08.009

Greenwald, A. G., McGhee, D. E., \& Schwartz, J. K. L. (1998). Measuring individual differences in implicit cognition: The implicit association test. Journal of Personality and Social Psychology, 74, 1464-1480.

Grieve, E., Fenwick, E., Yang, H. C., \& Lean, M. (2013). The disproportionate economic burden associated with severe and complicated obesity: A systematic review. Obesity Reviews, 14(11), 883-894. doi: 10.1111/obr.12059

Griffith, A. I. (1998). Insider / outsider: Epistemological privilege and mothering work. Human Studies, 21(4), 361-376. doi: 10.2307/20011213

Griffiths, P. (2008). Ethical conduct and nurse ethnographer: Consideration of an ethic of care. Journal of Research in Nursing, 13(4), 350-361. 
Grindel, M. E., \& Grindel, C. G. (2006). Nursing care of the person having bariatric surgery. Medsurg Nursing, 15(3), 129-145.

Gross, N. D., Cohen, J. I., Andersen, P. E., \& Wax, M. K. (2002). 'Defatting' tracheotomy in morbidly obese patients. Laryngoscope, 112(11), 1940 - 1944.

Guba, E. G. (1981). Criteria for assessing the trustworthiness of naturalistic inquiries. ECRJ, 29(2), 75-91.

Gunderson, C. G. (2011). Cellulitis: Definition, etiology, and clinical features. The American Journal of Medicine, 124(12), 1113-1122. doi: http://dx.doi.org/10.1016/j.amjmed.2011.06.028

Hales, C. (2012). The shifting boundaries of engagement when researching colleagues within one's own workplace. Paper presented at the Comtemporary Ethnography Across the Disciplines, Waikato University, New Zealand

Hammersley, M. (1998). Reading ethnographic research: A critical guide. (2 ed.). London: Longman.

Hammersley, M., \& Atkinson, P. (2007). Ethnography: Principles in practice (3rd ed.). London: Routledge.

Health and Disability Ethics Committees. (2005). Guidelines for completion of the national application form for ethical approval of a research project (NAF-2005 v1). Wellington: Ministry of Health.

Hebl, M., \& Xu, J. (2001). Weighing the care: Physician's reactions to the size of a patient. International Journal of Obesity, 25(8), 1246-1252.

Hebl, M., Xu, J., \& Mason, M. F. (2003). Weighing the care: Patients' perceptions of physician care as a function of gender and weight. International Journal of Obesity and Related Disorders, 27(2), 269-275. doi: http://dx.doi.org/10.1038/sj.ijo.802231

Hebl, M. R., Tickle, J., \& Heatherton, T. F. (2000). Awkward moments in interactions between non-stigmatised and stigmatised individuals. In T. F. Heatherton (Ed.), The social psychology of stigma (pp. 275-306). New York: Guildford Press.

Heijnders, M., \& Van Der Meij, S. (2006). The fight against stigma: An overview of stigmareduction strategies and interventions. Psychology, Health and Medicine, 11(3), 353363. doi: 10.1080/13548500600595327

Hennink, M., Hutter, I., \& Bailey, A. (2011). Qualitative research methods. London: SAGE Publications.

Heritage, J. (1984). Garfinkel and ethnomethodology. Cambridge: Polity.

Hertz, R. (Ed.). (1997). Reflexivity and voice. California: Thousand Oaks, Sage.

Heshka, S., \& Allison, D. B. (2001). Is obesity a disease? International Journal of Obesity and Related Disorders, 25(10), 1401-1404. doi: http://dx.doi.org/10.1038/sj.ijo.0801790

Higginbottom, G. M., Pillay, J. J., \& Boadu, N. Y. (2013). Guidance on performing focused ethnographies with an emphasis on healthcare research. The Qualitative Report, 18(Art 17), 1-16.

Higginbottom, G. M. A. (2011). The transitioning experiences of internationally-educated nurses into a Canadian health care system: A focused ethnography. BioMed Central Nursing, 10(4), 1-13. http://www.biomedcentral.com/1472-6955/10/14

Hirani, V., Zaninotto, P., \& Primatesta, P. (2008). Generalised and abdominal obesity and risk of diabetes, hypertension and hypertension-diabetes co-morbidity in England. Public Health Nutrition, 11(5), 521-527. doi: http://dx.doi.org/10.1017/S1368980007000845

Hoare, K. J., Buetow, S., Mills, J., \& Francis, K. (2013). Using an emic and etic ethnographic technique in a grounded theory study of information use by practice nurses in New 
Zealand. Journal of Research in Nursing, 18(8), 720-731. doi: 10.1177/1744987111434190

Hochschild, A. R. (1979). Emotion work, feeling rules, and social structure. American Journal of Sociology, 85(3), 551-575. doi: 10.2307/2778583

Hochschild, A. R. (1983). The managed heart. Commercialisation of human feeling. Berkley: University of California Press.

Holloway, I., \& Wheeler, S. (1995). Ethical issues in qualitative nursing research. Nursing Ethics, 2(3), 223-232. doi: 10.1177/096973309500200305

Hoppe, R., \& Ogden, J. (1997). Practice nurses' beliefs about obesity and weight related interventions in primary care. International Journal of Obesity, 21, 141-146.

Houghton, C., Casey, D., Shaw, D., \& Murphy, K. (2010). Ethical challenges in qualitative research: Examples from practice. Nurse Researcher, 18(1), 15-25.

Hubert, H. B., Feinleib, M., McNamara, P. M., \& Castelli, W. P. (1983). Obesity as an independent risk factor for cardiovascular disease: A 26-year follow-up of participants in the framingham heart study. Circulation, 67(5), 968-977. doi: 10.1161/01.cir.67.5.968

Human Rights Commission. (2011). Structural discrimination. The need for systematic change to achieve racial equality. Draft discussion paper for community consultation. Wellington: Human Rights Commission.

Hurst, S., Blanco, K., Boyle, D., Douglass, L., \& Wikas, A. (2004). Bariatric implications of critical care nursing. Dimensions of Critical Care Nursing, 23(2), 76-83.

Huschak, G., Busch, T., \& Kaisers, U. X. (2013). Obesity in anesthesia and intensive care. Best Practice and Research Clinical Endocrinology and Metabolism, 27(2), 247-260. doi: http://dx.doi.org/10.1016/j.beem.2013.02.001

Huttunen, R., \& Syrjänen, J. (2013). Obesity and the risk and outcome of infection. International Journal of Obesity, 37(3), 333-340. doi: http://dx.doi.org/10.1038/ijo.2012.62

Hyde, A. (1998). From mutual pretense awareness to open awareness: Single pregnant women's public encounters in an Irish context. Qualitative Health Research, 8(5), 634-643. doi: 10.1177/104973239800800505

Ide, P., Farber, E. S., \& Lautz, D. (2008). Perioperative nursing care of the bariatric surgical patient. Association of Operating Room Nurses. AORN Journal, 88(1), 30-54; quiz 55-38. doi: http://dx.doi.org/10.1016/j.aorn.2008.02.015

Jamadarkhana, S., Mallick, A., \& Bodenham, A. R. (2014). Intensive care management of morbidly obese patients. Continuing Education in Anaesthesia, Critical Care and Pain, 14(2), 73-78. doi: 10.1093/bjaceaccp/mkt040

James, N. (1989). Emotional labour: Skill and work in the social regulation of feelings. The Sociological Review, 37(1), 15-42. doi: 10.1111/j.1467-954X.1989.tb00019.x

James, N. (1992). Care = organisation + physical labour + emotional labour. Sociology of Health and Illness, 14(4), 488-509. doi: 10.1111/1467-9566.ep10493127

Jia, H., \& Lubetkin, E. I. (2005). The impact of obesity on health-related quality-of-life in the general adult US population. Journal of Public Health, 27(2), 156-164. doi: 10.1093/pubmed/fdi025

Jones, C., \& Jack, B. (1999). Dilemmas in the ethics of health care research. Professional Nurse, 14(7), 425-455.

Kanuha, V. K. (2000). 'Being native' verses 'going native': Conducting social work research as an insider. Social Work, 45(5), 439-447.

Karlsson, J., Taft, C., Sjöström, L., Torgerson, J. S., \& Sullivan, M. (2003). Psychosocial functioning in the obese before and after weight reduction: Construct validity and 
responsiveness of the obesity-related problems scale. International Journal of Obesity and Related Disorders, 27(5), 617-630. doi: http://dx.doi.org/10.1038/sj.ijo.0802272

Karnieli-Miller, O., Strier, R., \& Pessach, L. (2009). Power relations in qualitative research. Qualitative Health Research, 19(2), 279-289. doi: 10.1177/1049732308329306

Karppelin, M., Siljander, T., Vuopio-Varkila, J., Kere, J., Huhtala, H., Vuento, R., . . . Syrjänen, J. (2010). Factors predisposing to acute and recurrent bacterial nonnecrotizing cellulitis in hospitalized patients: A prospective case-control study. Clinical Microbiology and Infection, 16(6), 729-734. doi: 10.1111/j.14690691.2009.02906.x

Kells, K. (2005). Lessons learned: Morbid obesity patient care needs. The Kansas Nurse, 80(10), 9-14.

Kelly, M. P., \& May, D. (1982). Good and bad patients: A review of the literature and a theoretical critique. Journal of Advanced Nursing, 7(2), 147-156. doi: 10.1111/j.13652648.1982.tb00222.x

Klein, D., Najman, J., Kohrman, A. F., \& Munro, C. (1982). Patient characteristics that elicit negative responses from family physicians. Journal of Family Practice, 14, 881-888.

Knoblauch, H. (2005). Focused ethnography [30 paragraphs]. Forum: Qualitative Social Research, 6(3). http://www.qualitative-research.net.fqs/

Kodama, S., Horikawa, C., Fujihara, K., Yoshizawa, S., Yachi, Y., Tanaka, S., . . Sone, H. (2014). Quantitative relationship between body weight gain in adulthood and incident type 2 diabetes: A meta-analysis. Obesity Reviews, 15(3), 202-214. doi: $10.1111 /$ obr.12129

Kopelman, P. G., \& Finer, N. (2001). Reply: Is obesity a disease? International Journal of Obesity and Related Disorders, 25(10), 1405-1406. doi: http://dx.doi.org/10.1038/sj.ijo.0801791

Koro-Ljungberg, M., \& Greckhamer, T. (2005). Strategic turns labeled 'ethnography': From description to openly idealological productions of culture. Qualitative research, 5(3), 285-306.

Kotchen, T. A. (2010). Obesity-related hypertension: Epidemiology, pathophysiology, and clinical management. American Journal of Hypertension, 23(11), 1170-1178. doi: http://dx.doi.org/10.1038/ajh.2010.172

Kvale, S., \& Brinkman, S. (2008). Interviews: Learning the craft of qualitative research interviewing (2nd ed.). London: SAGE Publications.

La Piere, R. T. (1934). Attitudes vs. action. Social Forces, 12, 230-237.

Labaree, R. V. (2002). The risks of going 'observationalist': Negotiating the hidden dilemmas of being an insider participant observer. Qualitative research, 2(2), 97-122.

Laberge, M. A., Baum, T., Virayavanich, W., Nardo, L., Nevitt, M. C., Lynch, J., . . Link, T. M. (2012). Obesity increases the prevalence and severity of focal knee abnormalities diagnosed using 3T MRI in middle-aged subjects--data from the Osteoarthritis Initiative. Skeletal Radiology, 41(6), 633-641. doi: http://dx.doi.org/10.1007/s00256011-1259-3

Lathlean, J. (1996). Ethical issues for nursing research: A methodological focus. Nursing Times Research, 1(3), 175-183. doi: 10.1177/174498719600100304

Lawton, J. (1998). The disintegration of self: A study of patients in a day care service and a hospice in England. (PhD), University of Cambridge.

Lawton, J. (2000). The dying process: Patients' experiences of palliative care. London: Routledge.

Lee, J. J., \& Pinker, S. (2010). Rationales for indirect speech: The theory of the strategic speaker. Psychological Review, 117(3), 785-807. doi: http://dx.doi.org/10.1037/a0019688 
Lerner, G. H. (1996). Finding "face" in the preference structures of talk-in-interaction. Social Psychology Quarterly, 59(4), 303-321.

Lerner, M. (1980). The belief in a just world: Delusions. New York: Plenum Press.

Lewandowski, K., \& Lewandowski, M. (2011). Intensive care in the obese. Best Practice and Research Clinical Anaesthesiology, 25(1), 95-108. doi: http://dx.doi.org/10.1016/j.bpa.2010.12.003

Lewis, R. J., Cash, T. F., Jacobi, L., \& Bubb-Lewis, C. (1997). Prejudice toward fat people: The development and validation of the antifat attitudes test. Obesity Research, 5, 297 $-307$.

Li, S. (2002). Symbiotic niceness: A study of psychosocial care in palliative care settings. (Doctor of Philosophy), Goldsmith's College University of London, London.

Li, S. (2004). 'Symbiotic niceness': Constructing a therapeutic relationship in psychosocial palliative care. Social Science and Medicine, 58, 2571-2583.

Li, S. (2005). Doing criticism in'symbiotic niceness': A study of palliative care nurses' talk. Social Science and Medicine, 60, 1949-1959.

Lim, T. S., \& Bowers, J. W. (1991). Facework solidarity, approbation, and tact. Human Communication Research, 17(3), 415-450. doi: 10.1111/j.1468-2958.1991.tb00239.x

Lincoln, S. Y., Lynham, S. A., \& Guba, E. G. (2011). Paradigmatic controversies, contradictions, and emerging confluences revisited. In K. N. Denzin \& S. Y. Lincoln (Eds.), The SAGE handbook of qualitative research (4th ed., pp. 97-128). London: SAGE Publications.

Lincoln, Y. S., \& Guba, E. G. (1985). Naturalistic inquiriy. Beverly Hills: Sage.

Lincoln, Y. S., \& Guba, E. G. (2000). Paradigmatic controversies: Contradictions and emerging confluences. In K. N. Denzin \& S. Y. Lincoln (Eds.), Handbook of qualitative research (2nd ed.). Thousand Oaks: SAGE Publications.

Link, B. G., Castille, D. M., \& Stuber, J. (2008). Stigma and coercion in the context of outpatient treatment for people with mental illnesses. Social Science and Medicine, 67(3), 409-419. doi: 10.1016/j.socscimed.2008.03.015

Link, B. G., \& Phelan, J. C. (2001). Conceptualizing stigma. Annual Review of Sociology, 27, 363-385. doi: $10.2307 / 2678626$

Link, B. G., \& Phelan, J. C. (2006). Stigma and its public health implications. The Lancet, 367(9509), 528-529.

Link, B. G., Yang, L. H., Phelan, J. C., \& Collins, P. Y. (2004). Measuring mental illness stigma. Schizophrenia Bulletin, 30(3), 511-541.

Little, M., Jordens, C. F., Paul, K., Sayers, E., \& Sriskandarajah, D. (2000). Face, honor and dignity in the context of colon cancer. Journal of Medical Humanities, 21(4), 229-243. doi: 10.1023/A:1009077209274

Longhurst, R. (2005). Fat bodies: Developing geographical research agendas. Progress in human geography, 29(3), 247-259.

MacLean, L., Edwards, N., Garrard, M., Sims-Jones, N., Clinton, K., \& Ashley, L. (2009). Obesity, stigma and public health planning. Health Promotion International, 24(1), 88-93. doi: 10.1093/heapro/dan041

Maddox, G. L., \& Liederman, V. (1969). Overweight as a social disability with medical implications. Journal of Medical Education, 44, 214-220.

Madison, D. S. (2005). Critical ethnography: Method, ethics, and performance. California: Thousand Oaks.

Main, J. (2002). Management of relatives of patients who are dying. Journal of Clinical Nursing, 11(6), 794-801. doi: 10.1046/j.1365-2702.2002.00672.x

Marcus, G., \& Fischer, M. (1986). Antropology as cultural critique: An experiental moment in the human sciences. Chicago: The University of Chicago Press. 
Maroney, D., \& Golub, S. (1992). Nurses' attitudes towards obese persons and certain ethnic groups. Perceptual and Motor Skills, 75, 387-391.

Martino, J. L., Stapleton, R. D., Wang, M., Day, A. G., Cahill, N. E., Dixon, A. E., . . . Heyland, D. K. (2011). Extreme obesity and outcomes in critically ill patients. CHEST Journal, 140(5), 1198-1206. doi: 10.1378/chest.10-3023

McClure, R., \& Murphy, C. (2007). Contesting the dominance of emotional labour in professional nursing. Journal of Health organisation and management, 21(2), 101120.

McCrea, B. H., Wright, M. E., \& Murphy-Black, T. (1998). Differences in midwives' approaches to pain relief in labour. Midwifery, 14, 174-180.

McGinley, L. D., \& Bunke, J. (2008). Best practices for safe handling of the morbidly obese patient. Bariatric Nursing and Surgical Patient Care, 3(4), 255-260. doi: http://dx.doi.org/10.1089/bar.2008.9951

Medical Council of New Zealand. (2013). Good medical practice. Medical Council of New Zealand, Wellington.

Meerabeau, L. (1999). The management of embarrassment and sexuality in health care. Journal of Advanced Nursing, 29(6), 1507-1513. doi: 10.1046/j.13652648.1999.01039.x

Mental Health Commission. (1997). Discrimination against mental health consumers. Wellington, New Zealand.

Mercer, S., \& Tessier, S. (2001). A qualitative study of general practitioners' and practice nurses' attitudes to obesity management in primary care. Health Bulletin, 59, 248-253.

Merriam, S. B. (1998). Qualitative research and case study application in education. San Francisco: Jossey-Bass.

Merriam, S. B., Johnson-Bailey, J., Lee, M. Y., Kee, Y., Ntseane, G., \& Muhamad, M. (2001). Power and positionality: Negotiating insider/outsider status within and across cultures. International Journal of Lifelong Education, 20(5), 405-416. doi: $10.1080 / 02601370120490$

Merrill, E., \& Grassley, J. (2008). Women's stories of their experiences as overweight patients. Journal of Advanced Nursing, 64(2), 139-146.

Ministry of Health. (2003). Healthy eating- Healthy action: Oranga kai- Oranga pumau: A strategic framework. Ministry of Health, Wellington, New Zealand.

Ministry of Health. (2004). Tracking the obesity epidemic: New Zealand 1977-2003. Ministry of Health, Wellington, New Zealand.

Ministry of Health. (2008a). Body size technical report: Measurements and classifications in 2006/07 New Zealand health survey. Ministry of Health, Wellington, New Zealand.

Ministry of Health. (2008b). Health Targets: Moving towards healthier futures 2008/09- The results. Ministry of Health, Wellington, New Zealand.

Ministry of Health. (2009). Clinical guidelines for weight management in New Zealand adults. Ministry of Health, Wellington, New Zealand.

Ministry of Health. (2010). Implementing the clinical guidelines for weight management in New Zealand 2010/11: Implementation plan. Ministry of Health, Wellington, New Zealand.

Ministry of Health. (2013). New Zealand health survey: Annual update of key findings 2012/13. Ministry of Health, Wellington, New Zealand.

Ministry of Health. (2014). Health Targets. Retrieved 23/06/2014, 2014, from http://www.health.govt.nz/new-zealand-health-system/health-targets

Ministry of Health, \& University of Auckland. (2003). Nutrition and the burden of disease: New Zealand 1997-2001. Wellington, New Zealand Ministry of Health. 
Ministry of Justice. (1993). Human Rights Act. Wellington, New Zealand: New Zealand Government.

Mold, F., \& Forbes, A. (2013). Patients' and professionals' experiences and perspectives of obesity in health-care settings: A synthesis of current research. Health Expectations, 16(2), 119-142. doi: 10.1111/j.1369-7625.2011.00699.x

Morley, A. (2005). Minimum guidelines for intensive care nurse staffing in New Zealand. New Zealand Nurses' Organisation, Wellington.

Muir, M., Heese, G. A., McLean, D., Bodnar, S., \& Rock, B. L. (2007). Handling of the bariatric patient in critical care: A case study of lessons learned. Critical Care Nursing Clinics of North America, 19(2), 223-240. doi: http://dx.doi.org/10.1016/j.ccell.2007.02.010

Myers, A., \& Rosen, J. C. (1999). Obesity stigmatisation and coping: Relation to mental health symptoms, body image, and self-esteem. International Journal of Obesity, 23, 221-230.

Nelson, T. D. (Ed.). (2009). Handbook of prejudice, stereotyping, and discrimination. New York: Psychology Press.

Nordholm, L. A. (1980). Beautiful patients are good patients: Evidence for the physical attractiveness stereotype in first impression of patients. Social Science and Medicine, $14 A, 81-83$.

Nursing Council of New Zealand. (2012). Codes of conduct for nurses. Nursing Council of New Zealand, Wellington, New Zealand.

O'Reilly, C., \& Sixsmith, J. (2012). From theory to policy: Reducing harms associated with the weight-centered health paradigm. Fat Studies, 1(1), 97-113. doi: $10.1080 / 21604851.2012 .627792$

O'Reilly, K. (2009). Key concepts in ethnography. London: SAGE Publications.

Oliver, S., \& Redfern, S. J. (1991). Interpersonal communication between nurses and elderly patients: Refinement of an observation schedule. Journal of Advanced Nursing, 16(1), 30-38. doi: 10.1111/j.1365-2648.1991.tb01494.x

Oliveros, H., \& Villamor, E. (2008). Obesity and mortality in critically ill adults: A systematic review and meta-analysis. Obesity, 16(3), 515-521. doi: http://dx.doi.org/10.1038/oby.2007.102

Olson, C. L., Schumaker, H. D., \& Yawn, B. P. (1994). Overweight women delay medical care. Archives of Family Medicine, 3(10), 888-892.

Packer, J. (1990). Barriers to health care utilization: The effect of medical stigma of "obesity" on women: City University of New York.

Page, S. (2006). Never say die: A study of cardiopulmonary resuscitation and the National Health Service-comments from an ethnographic enquiry. Journal of Research in Nursing, 11(5), 423-431. doi: 10.1177/1744987106067461

Parahi, C. (2014). Pharmac won't fund prostate cancer drug One News TVNZ.

Parker, R., \& Aggleton, P. (2003). HIV and AIDS-related stigma and discrimination: A conceptual framework and implications for action. Social Science and Medicine, 57(1), 13-24. doi: http://dx.doi.org/10.1016/S0277-9536(02)00304-0

Patton, M. Q. (1990). Qualitative evaluation and research methods (2nd ed.). California: Sage Publications.

Pearson-Ceol, J. (2007). Literature review on the effects of obesity on knee osteoarthritis. Orthopaedic Nursing, 26(5), 289-292.

Pepper, A. C., \& Ruiz, S. Y. (2007). Acculturation's influence on antifat attitudes, body image and eating behaviors. Eating Disorders, 15(5), 427-447.

Peternelj-Taylor, C. (1989). The effects of patient weight on sex on nurses' perceptions: A proposed model of nurse withdrawal. Journal of Advanced Nursing, 14, 744-754. 
Petrich, B. (2000). Medical and nursing students' perceptions of obesity. Journal of Addictions Nursing, 12, 3-16.

Pharmaceutical Management Agency (PHARMAC). (2013). Introduction to PHARMAC. New Zealand Government, Wellington.

Phelan, J. C., Link, B. G., \& Dovidio, J. F. (2008). Stigma and prejudice: One animal or two? Social Science and Medicine, 67(3), 358-367. doi: 10.1016/j.socscimed.2008.03.022

Pickering, T. G., Hall, J. E., Appel, L. J., Falkner, B. E., Graves, J., Hill, M. N., . . Roccella, E. J. (2005). Recommendations for blood pressure measurement in humans and experimental animals: Part 1: Blood pressure measurement in humans: A statement for professionals from the subcommittee of professional and public education of the American Heart Association Council on high blood pressure research. Hypertension, 45(1), 142-161. doi: 10.1161/01.HYP.0000150859.47929.8e

Pinker, S. (2009). The evolutionary social psychology of off-record indirect speech acts International Journal on Humanistic Ideology, 2(1), 59-89.

Poon, M., \& Tarrant, M. (2009). Obesity: Attitudes of undergraduate student nurses and registered nurses. Journal of Clinical Nursing, 18(16), 2355-2365. doi: 10.1111/j.1365-2702.2008.02709.x

Power, S., \& Condon, C. (2008). Chemotherapy-induced alopecia: A phenomenological study. Cancer Nursing Practice, 7(7), 44-47.

Prentice, A. M., \& Jebb, S. A. (2001). Beyond body mass index. Obesity Reviews, 2(3), 141147. doi: 10.1046/j.1467-789x.2001.00031.x

Prior, L. (1987). Policing the dead: A sociology of the mortuary. Sociology, 21(3), 355-376.

Prospective Studies Collaboration. (2009). Body mass index and cause-specific mortality in 900000 adults: Collaborative analysis of 57 prospective studies. The Lancet, 373(9669), 1083-1096.

Puhl, R. M., \& Brownell, K. D. (2001). Bias, discrimination and obesity. Obesity Research, 9, 788-805.

Puhl, R. M., \& Brownell, K. D. (2003). Psychosocial origins of obesity stigma: Towards changing a powerful and pervasive bias. Obesity Reviews, 4, 212-227.

Puhl, R. M., \& Heuer, C. A. (2009). The stigma of obesity: A review and update. Obesity (Silver Spring, Md), 17, 941 - 964.

Puhl, R. M., Heuer, C. A., \& Brownell, K. D. (2010). Stigma and social consequences of obesity. In P. G. Kopelman, I. D. Caterson \& W. H. Dietz (Eds.), Clinical obesity in adults and children (3rd ed., pp. 25-40). Oxford: Wiley-Blackwell.

Puhl, R. M., Moss-Racusin, C. A., Schwartz, M. B., \& Brownell, K. D. (2008). Weight stigmatization and bias reduction: Perspectives of overweight and obese adults. Health Education Research, 23(2), 347-358. doi: 10.1093/her/cym052

Reed-Danahay, D. (2002). Turning points and textual strategies in ethnographic writing. Qualitative Studies in Education, 15(4), 421-425.

Regner, J. L., Kobayashi, L., \& Coimbra, R. (2012). Surgical strategies for management of the open abdomen. World Journal of Surgery, 36(3), 497-510. doi: 10.1007/s00268011-1203-7

Reinharz, S. (1997). 'Who am I? The need for a variety of selves in the field. In R. Hertz (Ed.), Reflexivity and voice. California: Thousand Oaks, SAGE Publications.

Richards, L. (2009). Handling qualitative data: A practical guide (2nd ed.). Thousand oaks. California: SAGE Publications.

Robinson, B. E., Bacon, L. C., \& O'Reilly, J. (1993). Fat phobia: Measuring, understanding, and changing anti-fat attitudes. International Journal of Eating Disorders, 14(4), 467480. doi: 10.1002/1098-108X(199312)14:4<467::AID-EAT2260140410>3.0.CO;2-J 
Robitaille, C., \& Saint-Jacques, M. (2009). Social stigma and the situation of young people in lesbian and gay stepfamilies. Journal of Homosexuality, 56(4), 421-442. doi: $10.1080 / 00918360902821429$

Rogge, M. M., Greenwald, M., \& Golden, A. (2004). Obesity, stigma, and civilized oppression. Advances in Nursing Science, 27(4), 301-315.

Roper, J. M., \& Shapira, J. (2000). Ethnography in nursing research. Thousand Oaks: SAGE Publications.

Rose, M. A., Drake, D., Baker, G., Watkins, F., Waters, W., \& Pokorny, M. (2008). Caring for morbidly obese patients: Safety considerations for nurse administrators. Nursing Management, 39(11), 47-50.

Roth, J., Qiang, X., Marban, S. L., Redelt, H., \& Lowell, B. C. (2004). The obesity pandemic: Where have we been and where are we going? Obesity Research, 12(Supplement), 88S-1101S.

Rothblum, E. D. (2012). Why a journal on fat studies? Fat Studies, 1(1), 3-5. doi: $10.1080 / 21604851.2012 .633469$

Rumsey, N., \& Harcourt, D. (2004). Body image and disfigurement: Issues and interventions. Body Image, 1(1), 83-97. doi: http://dx.doi.org/10.1016/S1740-1445(03)00005-6

Russell, N., \& Carryer, J. (2013). Living large: The experiences of large-bodied women when accessing general practice services. Journal of Primary Health Care, 5(3), 199-205.

Sakr, Y., Elia, C., Mascia, L., Barberis, B., Cardellino, S., Livigni, S., . . . Ranieri, V. M. (2012). Being overweight or obese is associated with decreased mortality in critically ill patients: A retrospective analysis of a large regional Italian multicenter cohort. Journal of Critical Care, 27(6), 714-721. doi: http://dx.doi.org/10.1016/j.jcrc.2012.08.013

Sánchez-de-la-torre, M., Mediano, O., Barceló, A., Piérola, J., de la Peña, M., Esquinas, C., . . . Barbé, F. (2012). The influence of obesity and obstructive sleep apnea on metabolic hormones. Sleep and Breathing, 16(3), 649-656. doi: http://dx.doi.org/10.1007/s11325-011-0552-7

Sanday, P. R. (1979). The ethnographic paradigms. Administrative Science Quarterly, 24, 527-538.

Sarantakos, S. (1998). Social research (2nd ed.). Basingstoke, UK: MacMillan.

Sassi, F. (2010). Obesity and the economics of prevention: Fit not fat: Organisation of Economic Co-operation and Development.

Savage, J. (2006). Ethnographic evidence: The value of applied ethnography in healthcare. Journal of Research in Nursing, 11(5), 383-393. doi: 10.1177/1744987106068297

Sayce, L. (1998). Stigma, discrimination and social exclusion: What's in a word? Journal of Mental Health, 7(4), 331-343.

Scambler, G. (2009). Health-related stigma. Sociology of Health and Illness, 31(3), 441-455.

Schwartz, M. B., Chambliss, H. O., Brownell, K. D., Blair, S. N., \& Billington, C. (2003). Weight bias among health professionals specialising on obesity. Obesity Research, 11(9), 1033-1039.

Schwartz, M. B., Vartanian, L. R., Nosek, B. A., \& Brownell, K. D. (2006). The influence of one's own body weight on implicit and explicit anti-fat bias. Obesity, 14(3), 440-447. doi: http://dx.doi.org/10.1038/oby.2006.58

Scott, S. D., \& Pollock, C. (2008). The role of nursing culture in shaping research utilisation behaviours. Journal in Nursing and Health, 31(4), 298-309.

Seale, C. (1999). The quality of qualitative research. London: SAGE Publications.

Seale, C., \& Silverman, D. (1997). Ensuring rigour in qualitative research. European Journal of Public Health, 7, 379-384.

Seymour-Smith, C. (1986). Macmillan dictionary of anthropology. London: Macmillan. 
Shattell, M. (2002). Eventually it will be over: The dialectic between confinement and freedom in the phenomenal world of the hospitalised patient. In S. Thomas \& H. Pollio (Eds.), Listening to patients: A phenomenologiccal approach to nursing research and practice (pp. 214-236). New York: Springer.

Shattell, M. (2004). Nurse-patient interaction: A review of the literature. Journal of Clinical Nursing, 13, 714-722.

Shenton, A. K. (2004). Strategies for ensuring trustworthiness in qualitative research projects. Education for Information, 22, 63-75.

Shields, M., Carroll, M., \& Ogden, C. (2011). Adult obesity prevalence in Canada and the United States. NCHS Data Briefs, 56, 1-8.

Siavash, M., Sadeghi, M., Salarifar, F., Amini, M., \& Shojaee-moradie, F. (2009). Comparison of body mass index and waist/height ratio in predicting definite coronary artery disease. Annals of Nutrition and Metabolism, 53(3-4), 162-166.

Silverman, D. (2013). Doing qualitative reseach: A practical handbook (4th ed.). London: SAGE Publications.

Simmons, M. (2007). Insider ethnography: Tinker, tailor, researcher or spy? Nurse Researcher, 14(4), 7-17.

Siyam'kela. (2003). HIV/AIDS stigma indicators. A tool for measuring the progress of HIV/AIDS stigma mitigation. Cape Town, Policy Project, South Africa.

Smallwood, A. (2009). Cardiac assessment teams: A focussed ethnography of nurses' roles. British Journal of Cardiac Nursing, 4(3), 132-138.

Smith, D. E. (2005). Institutional ethnography: A sociology for people. Oxford: AltaMira Press.

Smith, P. (1991). The nursing process: Raising the profile of emotional care in nurse training. Journal of Advanced Nursing, 16(1), 74-81. doi: 10.1111/j.1365-2648.1991.tb01500.x

Smith, P. (1992). The emotional labour of nursing: How nurses care. London: MacMillian.

Smith, P., \& Gray, B. (2001a). Emotional labour of nursing revisited: Caring and learning 2000. Nurse Education in Practice, 1(1), 42-49. doi: http://dx.doi.org/10.1054/nepr.2001.0004

Smith, P., \& Gray, B. (2001b). Reassessing the concept of emotional labour in student nurse education: Role of link lecturers and mentors in a time of change. Nurse Education Today, 21(3), 230-237. doi: http://dx.doi.org/10.1054/nedt.2001.0541

Sorensen, R., \& Iedema, R. (2009). Emotional labour: Clinicians' attitudes to death and dying. Journal of Health Organization and Management, 23(1), 5-22. doi: http://dx.doi.org/10.1108/14777260910942524

Soylu, A. C., Levent, E., Sariman, N., Yurtlu, S., Alparslan, S., \& Saygi, A. (2012). Obstructive sleep apnea syndrome and anthropometric obesity indexes. Sleep and Breathing, 16(4), 1151-1158. doi: http://dx.doi.org/10.1007/s11325-011-0623-9

Sparks, L., Travis, S. S., \& Thompson, S. R. (2005). Listening for the communicative signals of humor, narratives, and self-disclosure in the family caregiver interview. Health and Social Work, 30(4), 340-343.

Sparks-Bethea, L., Travis, S. S., \& Pecchioni, L. (2000). Family caregivers' use of humor in conveying information about caring for dependent older adults. Health Communication, 12(4), 361-376. doi: 10.1207/S15327027HC1204_3

Spier, J. A., \& Wood, A. (2010). Building a therapeutic alliance in brief therapy: The experience of community mental health nurses. Archives of Psychiatric Nursing, 24(6), 373-386.

Spiers, J. A. (1998). The use of face work and politeness theory. Qualitative Health Research, 8(1), 25-47. doi: 10.1177/104973239800800103

Spradley, J. P. (1979). The ethnographic interview. New York: Holt, Rinehart and Winston. 
Staden, H. (1998). Alertness to the needs of others: A study of the emotional labour of caring. Journal of Advanced Nursing, 27(1), 147-156. doi: 10.1046/j.13652648.1998.00498.x

Stoneman, S. (2012). Ending fat stigma: Precious, visual culture, and anti-obesity in the "fat moment'. The Review of Education, Pedagogy, and Cultural Studies, 34, 197-207. doi: 10.1080/10714413.2012.687297

Strauss, A. L., \& Corbin, J. (1990). Basics of qualitative research. Thousand Oaks: SAGE Publications.

Street, A. F. (1992). Inside nursing: A critical ethnography of clinical nursing practice

Stuber, J., Galea, S., \& Link, B. G. (2008). Smoking and the emergence of a stigmatized social status. Social Science and Medicine, 67(3), 420-430. doi: 10.1016/j.socscimed.2008.03.010

Stuber, J., \& Meyer, I. (2008). Stigma, prejudice, discrimination and health. Social Science and Medicine, 67, 351-357. doi: 10.1016/j.socimed.2008.03.023

Stuber, J., \& Schlesinger, M. (2006). Sources of stigma for means-tested government programs. Social Science \& Medicine, 63(4), 933-945. doi: http://dx.doi.org/10.1016/j.socscimed.2006.01.012

Stunkard, A. J., \& Sorensen, R. (1993). Obesity and socio-economic status- A complex relation. New England Journal of Medicine, 329, 1036-1037.

Subramaniam, N., Nandhakumar, J., \& Baptista, J. (2013). Exploring social network interactions in enterprise systems: The role of virtual co-presence. Information Systems Journal, 23(6), 475-499. doi: 10.1111/isj.12019

Tanner, J., \& Timmons, S. (2000). Backstage in the theatre. Journal of Advanced Nursing, 32(4), 975-980. doi: 10.1046/j.1365-2648.2000.t01-1-01564.x

Tay, L. H., Hegney, D., \& Ang, E. (2011). Factors affecting effective communication between registered nurses and adult cancer patients in an inpatient setting: A systematic review. International Journal of Evidence-based Healthcare, 9, 151-164.

Teachman, \& Brownell, K. D. (2001). Implicit anti-fat bias among health professionals: Is anyone immune? International Journal of Obesity, 25, 1525 - 1531.

Teachman, B. A., Gapinski, K. D., \& Brownell, K. D. (2001). Stigma of obesity: Implicit attitudes and stereotypes. Paper presented at the Society for Personality and Social Psychology, San Antonio, Texas.

Teachman, B. A., Gapinski, K. D., Brownell, K. D., Rawlins, M., \& Jeyaram, S. (2003). Demonstrations of implicit anti-fat bias: The impact of providing causal information and evoking empathy. Health Psychology, 22(1), 68-78. doi: http://dx.doi.org/10.1037/0278-6133.22.1.68

Tedlock, B. (2000). Ethnography and ethnography representation. In N. K. Denzin \& Y. S. Lincoln (Eds.), Handbook of qualitative research (2nd ed., pp. 455-486). Thousand Oaks, California: SAGE Publications.

Theodosius, C. (2008). Emotional labour in health care: The unmanaged heart of nursing. London: Routledge.

Thomas, J. (1993). Doing critical ethnography. California: Sage Publications.

Thomas, S. L., Hyde, J., Karunaratne, A., Herbert, D., \& Komesaroff, P. A. (2008). Being 'fat' in today's world: A qualitative study of the lived experiences of people with obesity in Australia. Health Expectations, 11(4), 321-330. doi: 10.1111/j.13697625.2008.00490.x

Thomson, D. (2011). Ethnography: A suitable approach for providing an inside perspective on the everyday lives of health professionals. International Journal of Therapy and Rehabilitation, 18(1), 10-17. 
Thupayagale-Tshweneagae, G. (2008). Psychosocial effects experienced by grandmothers as primary caregivers in rural Botswana. Journal of Psychiatric and Mental Health Nursing, 15, 351-356.

Tjepkema, M. (2008). Measured obesity. Adult obesity in Canada: Measured height and weight. Nutrition: Findings from the Canadian Community Health Survey 2004. Issue I. Canada.

Trimble, T. (2007). Outsize patients- A big nursing challenge. Retrieved February 102014 , 2014, from http://enw.org/Obese.htm

Tsai, A. G., Williamson, D. F., \& Glick, H. A. (2011). Direct medical cost of overweight and obesity in the USA: A quantitative systematic review. Obesity Reviews, 12(1), 50-61. doi: 10.1111/j.1467-789X.2009.00708.x

Tuhiwai Smith, L. (2001). Decolonising methodologies: Research and indigenous people (1st ed.). Dunedin, New Zealand: University of Otago Press.

Tuhiwai Smith, L. (2010). Social justice, transformation and indigenous methodologies. Paper presented at the Contemporary Ethnography Across the Disciplines (CEAD), Waikato University, Waikato.

Turnock, C., \& Gibson, V. (2001). Validity in action research: A discussion on theoretical and practice issues encountered whilst using observation to collect data. Journal of Advanced Nursing, 36(3), 471-477. doi: 10.1046/j.1365-2648.2001.01995.x

Tzeng, W. C., Yang, C. I., \& Tzeng, N. S. (2010). The inner door: Towards an understanding of suicidal patients. Journal of Clinical Nursing, 19(9-10), 1396-1404.

UNAIDS. (2000). Protocol for the identification of discrimination against people living with HIV. Geneva, UNAIDS.

Van Brakel, W. H. (2006). Measuring health-related stigma- A literature review. Psychology, Health and Medicine, 11(3), 307-334. doi: 10.1080/13548500600595160

Van Brakel, W. H., Anderson, A. M., Mutatkar, R. K., Bakirtzief, Z., Nicholls, P. G., Raju, M. S., \& Das-Pattanayak, R. K. (2006). The participation scale: Measuring a key concept in public health. Disability and Rehabilitation, 28(4), 193-203. doi: 10.1080/09638280500192785

Van Maanen, J. (1979). Qualitative methodology. London: SAGE Publications.

Van Maanen, J. (1995). Representation in ethnography. Thousand Oaks, California: Sage Publications.

Van Maanen, J. (2011). Ethnography as work: Some rules of engagement. Journal of Management Studies, 48(1), 218-234. doi: 10.1111/j.1467-6486.2010.00980.x

Van Manen, M. (2014). Phenomenology of practice: Meaning-giving methods in phenomenological research and writing. California: Left Coast Press.

Varon, J., \& Marik, P. (2001). Management of the obese critically ill patient. Critical Care Clinics, 17(1), 1-14.

Villareal, D. T., Apovian, C. A., Kushner, R. F., \& Klien, S. (2005). Obesity in older adults: Technical review and position statement of the American Society for Nutrition and NAASO. The Obesity Society. Obesity Research, 13(11), 1849-1863.

Villavicencio, M. A., Sundt, T. A., Daly, R. C., Dearani, J. A., McGregor, C. G., Mullany, C. J., . . Schaff, H. V. (2007). Cardiac surgery in patients with a body mass index of 50 or greater. Annals of Thoracic Surgery, 83, 1403-1411.

Wacquant, L. (2002). Scrutinizing the street: Poverty, morality, and the pitfalls of urban ethnography. American Journal of Sociology, 107(6), 1468-1532. doi: $10.1086 / 340461$

Wadden, T. A., Butryn, M. L., Sarwer, D. B., Fabricatore, A. N., Crerand, C. E., Lipschutz, P. E., . . . Williams, N. N. (2006). Comparison of psychosocial status in treatment- 
seeking women with class III vs. class I-II obesity. Obesity, 14(3S), 90S-98S. doi: http://dx.doi.org/10.1038/oby.2006.288

Wadden, T. A., \& Didie, E. (2003). What's in a name? Patients' preferred terms for describing obesity. Obesity Research, 11(9), 1140-1146. doi: 10.1038/oby.2003.155

Wald, A. (2007). Fecal incontinence in elderly and institutionalized patients. In C. Ratto, G. B. Doglietto, A. Lowry, L. Pahlman \& G. Romano (Eds.), Fecal incontinence (pp. 317-323). Milan: Springer.

Walker, K. (1997). Cutting edges: Deconstructive inquiry and the mission of the border ethnographer. Nursing Inquiry, 4(1), 3-13. doi: 10.1111/j.1440-1800.1997.tb00131.x

Walsh, D. (1998). Doing ethnography. In C. Seale (Ed.), Research society and culture (pp. 217-232). Thousand Oaks: SAGE Publications.

Wann, M. (2009). Forewords. In E. Rothblum \& S. Solovay (Eds.), The fat studies reader. New York: New York University Press.

Watson, L., Oberle, K., \& Deutscher, D. (2008). Development and psychometric testing of the nurses' attitudes toward obesity and obese patients (NATOOPS) scale. Research in nursing and health, 31(6), 586-593.

Westbrook, M. T., \& Mitchel, R. A. (1979). Changes in sex-role stereotypes from health to illness. Social Science and Medicine, 13A, 279-302.

Westerly, B. D., \& Dabbagh, O. (2011). Morbidity and mortality characteristics of morbidly obese patients admitted to hospital and intensive care units. Journal of Critical Care, 26(2), 180-185. doi: http://dx.doi.org/10.1016/j.jcrc.2010.09.005

Wilkinson, S. (1991). Factors which influence how nurses communicate with cancer patients. Journal of Advanced Nursing, 16, 677-688.

Willis, K., \& Elmer, S. (2007). Society, culture, and health: An introduction to sociology for nurses. Melbourne: Oxford University Press.

Wilson, W. J., \& Chaddha, A. (2009). The role of theory in ethnographic research. Ethnography, 10(4), 549-564. doi: 10.1177/1466138109347009

Wing-Chung, H. (2008). Writing experience: Does ethnography convey a crisis of representation, or an ontological break with the everyday world? Canadian Sociology Association, 45(4), 343-365.

Winkelman, C., \& Maloney, B. (2005). Obese ICU patients: Resource utilization and outcomes. Clinical Nursing Research, 14(4), 303-323. doi: $10.1177 / 1054773805275288$

Withrow, D., \& Alter, D. A. (2011). The economic burden of obesity worldwide: A systematic review of the direct costs of obesity. Obesity Reviews, 12(2), 131-141. doi: 10.1111/j.1467-789X.2009.00712.x

Wittenberg-Lyles, E., Goldsmith, J., \& Ragan, S. (2011). The shift to early palliative care: A typology of illness journeys and the role of nursing. Clinical Journal of Oncology Nursing, 15(3), 304-310. doi: 10.1002/cncr.21011

Wolcott, H. F. (1973). The man in the principal's office. New York: Holt, Rinehart and Winston, Inc.

Wolcott, H. F. (1995). The art of fieldwork. Walnut Creek, California: AltaMira Press.

Wood, L. A., \& Kroger, R. O. (1991). Politeness and forms of address. Journal of Language and Social Psychology, 10(3), 145-168. doi: 10.1177/0261927x91103001

World Health Organisation. (2000). Obesity: Preventing and managing the global epidemic. Geneva: WHO.

World Health Organisation Expert Consultation. (2004). Appropriate body-mass index for Asian populations and its implications for policy and intervention strategies. The Lancet, 363(9403), 157-163. 
Wright, J. (1998). Female nurses' perceptions of acceptable female body size: An exploratory study. Journal of Clinical Nursing, 7(4), 307-315. doi: 10.1046/j.13652702.1998.00150.x

Yaegashi, M., Jean, R., Zuriqat, M., Noack, S., \& Homel, P. (2005). Outcome of morbid obesity in the intensive care unit. Journal of Intensive Care Medicine, 20(3), 147-154. doi: $10.1177 / 0885066605275314$

Young, L., \& Powell, B. (1985). The effects of obesity on the clinical judgements of mental health professionals. Journal of Clinical Nursing, 7, 307-315.

Zapf, D. (2002). Emotion work and psychological well-being: A review of the literature and some conceptual considerations. Human Resource Management Review, 12(2), 237268. doi: http://dx.doi.org/10.1016/S1053-4822(02)00048-7

Zuzelo, P. R., \& Seminara, P. (2006). Influence of registered nurses' attitudes toward bariatric patients on educational programming effectiveness. The Journal of Continuing Education in Nursing, 37(2), 65-73. 


\section{APPENDICES}

\section{Appendix 1: Research Advisory Group for Māori consent}

\section{MAORI PARTNERSHIP BOARD}

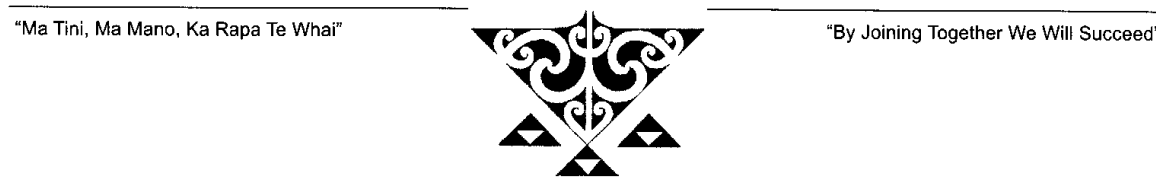

RESEARCH ADVISORY GROUP MAORI (RAG-M)

$15^{\text {th }}$ April 2009

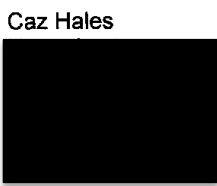

RAGM 2009/39 - Letter of Endorsement

Dear Caz

Tēna koe

On behalf of the Research Advisory Group (Māori) I write in relation to your study entitled "What is the culture of care for morbidly obese patients in intensive care settings?"

You have supplied a RAGM coversheet and copy of the application form for Ethical Approval which we understand you have submitted but have not yet received approval.

Our reading of your proposal characterises the research as:

- A small qualitative study with perhaps 8 patients as participants, who will be observed

- A study involving interviews with ICU staff, up to 90 nurses some of whom will be Māori

- A study in which, given the entrance criteria, Māori participants are probable, albeit in small numbers

We note that:

- you will seek approval from the relevant Health and Disability Ethics Committee; 
- you will advise the Research Committee of the CCDHB of the conduct of this research

- you have advised Victoria University.

Our assessment of this research is that it poses a relatively low risk in Māori terms. However, given the possibility of Māori obese ICU patients as participants, and likelihood of Māori ICU nurses, we would expect that:

- you would ensure that any Mãori who participates is well informed and supported, including their whānau as appropriate;

- you would recognise any cultural expectations and seek to meet these expectations responsively.

Whānau Care Services: You will be aware that WCS operate within the Wellington Regional Hospital. WCS have a responsibility to both patients of the hospital and staff. Given that this research may operate within the hospital campus we would expect that you:

- advise WCS of the operation of this research project

- negotiate with WCS the nature of their support

- Notify us of the arrangements which are in place.

On confirmation that the expectations specified above are understood and accepted by you we will be happy to endorse your research proposal. Please confirm these details with the RAG-M secretary, Ms Vanessa Mill.

We recognise that this small study may have implications for Măori who are cared for in ICU in the future and thank you for consulting with RAGM. We wish you well in your study.

Naku noa na,

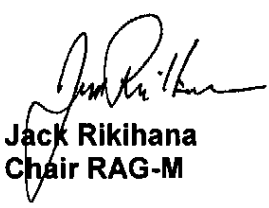




\section{Appendix 2: National Ethics approval letter}

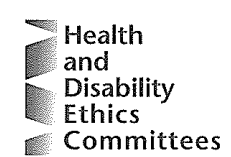

14 October 2009

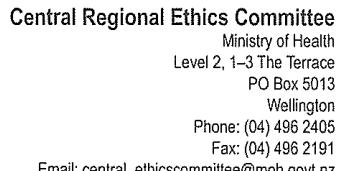

[Amendment to letter dated 23 September 2009]

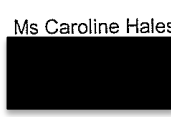

Dear Ms Caroline Hales

CEN/09/06/033 - Critical Ethnography: What is the Culture of Care of Bariatric Patients in the Intensive Care Setting?

The above study has been given ethical approval by the Central Regional Ethics Committee. Approved Documents

- Participant Information Sheet, Version 2, Dated 12/08/2009

- Nurse Participation - Observation Information Sheet, Version 2, Dated 12/08/2009

- Relative/Friend/Whanau Information Sheet, Version 2, Dated 12/08/2009

- Assent Form, Version 2, dated 12/08/2009

- Patient Consent Form, Version 2, Dated12/08/2009

- Nurse Participation-Observation Consent Form, Verson 2, dated 12/08/2009

Accreditation

The Committee involved in the approval of this study is accredited by the Health Research Council and is constituted and operates in accordance with the Operational Standard for Ethics Committees, April 2006.

Progress Reports
The study is approved until 01/11/2011. The Committee will review the approved application annually and notify the Principal Investigator if it withdraws approval. It is the Principal Investigator's responsibility to forward a progress report covering all sites prior to ethical review of the project in23/09/2010. The report form is available on http://www ethicscommittees.health.govt.nz. Please note that fallure to provide a progress report may result in the withdrawal of ethical approval. A final report is also required at the conclusion of the study.

\section{Amendments}

It is also a condition of approval that the Committee is advised if the study does not commence, or is altered in any way, including all documentation eg advertisements, letters to prospective participants.

Please quote the above ethics committee reference number in all correspondence.

The Principal Investigator is responsible for advising any other study sites of approvals and all other correspondence with the Ethics Committee.

It should be noted that Ethics Committee approval does not imply any resource commitment or administrative facilitation by any healthcare provider within whose facility the research is to be carried out Where applicable, authority for this must be obtained separately from the appropriate manager within the organisation.

Yours sincerely

sscott

Sonia Scott

Central Regional Ethics Committee Administrator

Email: sonia_scott@moh.govt.nz

Administered by the Ministry of Health $\quad$ Approved by the Heath Research Council $\quad$ hitp://iwww.ethicscommittes.health.govt.nz




\section{Appendix 3: Amended National Ethics approval letter}

$$
\begin{aligned}
& \text { Health } \\
& \text { and } \\
& \text { Disability } \\
& \text { Ethics } \\
& \text { Committees }
\end{aligned}
$$

24 February 2010

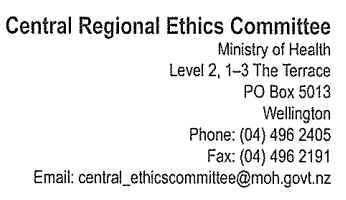

Caroline Hales

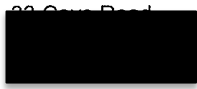

Dear Caroline Hales

CEN/09/06/033 - Critical Ethnography: What is the Culture of Care of Bariatric Patients in the Intensive Care Setting?

- Request to interview other health professionals working in the domain of intensive care alongside the nursing team

Thank you for submitting the above amendment, which was considered by the Acting Chairperson of the Central Regional Ethics Committee under delegated authority and approved.

Please quote the above ethics committee reference number in all correspondence.

Yours sincerely

Sonia Scott

Administrator

Central Regional Ethics Committee

Email: sonia_scott@moh.govt.nz 


\section{Appendix 4: Nurse study information sheet}

\section{Nurse Participation- Observation Information Sheet}

\begin{tabular}{|l|l|}
\hline Principal Investigator: & Contact Details: \\
\hline $\begin{array}{l}\text { Caz Hales } \\
\text { PhD student / researcher } \\
\text { Registered Nurse }\end{array}$ & \\
& \\
\hline
\end{tabular}

TITLE:

How do nurses care for very overweight patients in the intensive care setting?

\section{INTRODUCTION:}

You are invited to take part in a study which looks at how the nurses within Intensive Care Unit care for very overweight patients. If you would like to take part in the study, I will discuss the details with you prior to commencement of the proposed study period. You are under no obligation to take part and as such your employment or future employment opportunities will not be affected.

\section{ABOUT THE STUDY}

The aim of the study is to examine the everyday activities, social interactions and influences within the intensive care setting in which nurses' care for very overweight patients.

All patients who are admitted to

Intensive Care Unit who have a BMI $\geq 40$ $\mathrm{kg} / \mathrm{m} 2$ (obesity score based on height and weight), receiving non-weight loss care and is expected to remain in the unit for more than twelve hours have been considered for the study. Nurses who provide the care to that patient will be observed whilst providing bedside care. All nurses within Wellington's Intensive Care Unit will be involved in the study unless they choose not to participate.

The study will take place within the Intensive Care Unit at ospital over a three to four month period between November 2009 and February 2010. During the study, I will be observing the nursing team as they provide care for the very overweight patient throughout their intensive care admission and writing notes on what I see. The focus of the observation will be on how the nursing team interact with the patient, other nurses and health professionals to provide appropriate levels of care to support the patient's recovery. Additionally, I will be focussing on what resources are used and what resources are needed, if not available, to provide care that is supportive of the patient's individual needs. At no point during the study will conversations overheard during periods of observation be recorded and used as part of the data collection.

To provide an understanding of the individual needs of the patient and how the nurses have interpreted and planned that care I will be requesting to read the patient's medical notes and daily charts. The information required from the medical notes will be demographics (age, 
ethnicity, gender etc), past medical history and current health issues, reasons for admission to the unit, initial treatment plan and any limitations of treatment regimes/ modalities.

All information taken from the patient's notes will be written in note form and coded so that they remain unidentifiable. Any identifying information will not be included in the notes taken. All observation notes and medical information will be coded and stored in a secure and locked environment.

As part of the study, you will be invited to be interviewed so that you can provide further insight and understanding into how you care for very overweight patients on a daily basis. Being interviewed as part of the study is optional and does not effect your participation in the study as a whole. These interviews will be audio-taped, transcribed and coded so that any information specific to you or the patient will be unidentifiable. Should an identifiable detail be discussed during the interview then this will be removed from the data collection. Again, the data collected from the interviews will be coded and all audiotapes and written notes will be stored in a secure and locked environment.

After the study has been completed and the results have been published the data collected will be stored for ten years in a locked and secured environment and then destroyed.

\section{BENEFITS RISKS AND SAFETY}

There are no direct or immediate benefits of taking part in the study as the study is based on the observation of the care that you provide to the very overweight patient. However, your participation and the knowledge gained from observing your care of very overweight patients will hopefully benefit future patients admitted to the intensive care setting. Additionally, the study has the potential to highlight what resources are needed to ensure that nurses can safely provide care to this patient group.

The potential risk of the study is that it may make you feel self conscious about your nursing practices and subsequently become more reflective about caring for very overweight patients. Should you decide to take part in the interview process then time of approximately one hour during your work hours may pose some inconveniences. However, every effort will be made to select an appropriate time which minimises this inconvenience. During the interview any information given regarding how you care for or feel about caring for very overweight patients will be strictly confidential and not used in any capacity to report to management or for performance review processes.

The inclusion and exclusion criteria for the study are:

Patient Inclusion

1. A BMI $\geq 40 \mathrm{~kg} / \mathrm{m} 2$

2. Admission to ICU for reasons other than bariatric (weight loss) surgery

3. Is expected to remain in ICU for more than twelve hours

4. Consents to study participation

Patient Exclusion

1. Admitted to ICU following elective bariatric (weight loss) surgery

2. Is expected to be discharged from ICU within twelve hours

3. Does not consent to study participation

Nurse Inclusion

1. All intensive care nurses who care for the patient enrolled in the study who consents to be a study participant 
Nurse Exclusion

1. Any intensive care nurse who cares for the patient enrolled in the study who does not consent to be a study participant

Taking part in the study will not cost you anything. Additionally, the researcher is not in a position to provide payment or incentives to participants for their participation in the study.

\section{PARTICIPATION}

Your participation is entirely voluntary (your choice). You do not have to take part in this study, and if you choose not to take part this will not affect your employment or future employment opportunities.

If you do agree to take part you are free to withdraw from the study at any time, without having to give a reason and this will in no way affect your future employment opportunities.

\section{GENERAL}

Should you require further information about the study this can be provided by myself or Professor Jo Ann Walton (Research Supervisor). Contact details: jo.walton@,vuw.ac.nz Ph 04 4636135 .

Should you wish to have a friend, family or whanau support to help you understand the risks and/or benefits of this study and any other explanation you may require then please let me know so that I can ensure that they are present during all of our conversations about the study.

If you have any questions or concerns about your rights as a participant in this research study you can contact an independent health and disability advocate. This is a free service provided under the Health and Disability Commissioner Act.

Telephone: (NZ wide) 0800555050

Free Fax (NZ wide): 080027877678 (0800 2 SUPPORT)

Email (NZ wide): advocacy@hdc.org.nz"

Alternatively, you can contact The Central Regional Ethics Committee who have approved the research study:

Telephone: (04) 4962405

Email: central_ethicscommittee@moh.govt.nz

\section{CONFIDENTIALITY}

No material which could personally identify you will be used in any reports on this study. Following the examination of the study audiotapes will either be returned to the participant or destroyed as requested by the participant.

Persons identified as having access to confidential information are:

1. Caz Hales- Principal investigator

2. Professor Jo Ann Walton- Researcher supervisor (Head of Graduate School, Nursing, Midwifery and Health)

3. Confidential typist if needed to transcribe data

$$
\text { How do nurses care for very overweight patients within the intensive care setting? }
$$




\section{RESULTS}

A significant delay may occur between data collection and publication of the results due to the nature of the research. However, participants can receive information regarding the outcomes of the study should they wish by contacting myself. The results of the study will be published within an appropriate medical/ nursing journal and submitted as part of a $\mathrm{PhD}$ thesis to Victoria University of Wellington.

\section{STATEMENT OF APPROVAL}

This study has received ethical approval from the Central Regional Ethics Committee.

The Charge Nurse Manager and Medical Director of given permission for this study to be carried out.

Please feel free to contact the researcher if you have any questions about this study.

How do nurses care for very overweight patients within the intensive care setting? Version 2 


\section{Appendix 5: Nurse consent form}

\section{Nurse Participation-observation Consent Form}

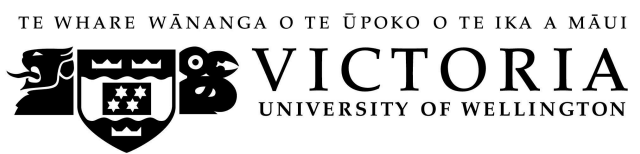

Title: What is the culture of care for very overweight patients in the intensive care setting?

Principal Investigator: Caroline Hales

Participant's Name

I have read and I understand the information sheet dated for volunteers taking part in the study designed to observe and interview nurses caring for very overweight patients within the intensive care unit. I have had the opportunity to discuss this study. I am satisfied with the answers I have been given.

I have had the opportunity to use whanau support or a friend to help me ask questions and understand the study.

I understand that taking part in this study is voluntary (my choice) and that I may withdraw from the study at any time and this will in no way affect my future employment.

I understand that my participation in this study is confidential and that no material which could identify me will be used in any reports on this study.

I have had time to consider whether to take part.

I consent to my interview being audio-taped.

YES/NO

I would like to read the interview transcript to verify what was said in the interview YES/NO

Following the study examination I would like my audiotape to be:

RETURNED TO ME/ DESTROYED BY THE RESEARCHER

I wish to receive a copy of the results.

YES/NO

I have been advised that a significant delay may occur between data collection and publication of the results.

Version 2

Page 1

$27 / 06 / 2014$ 
I (full name) hereby consent to take part in this study.

Date:

Signature:

Participant

Researcher: Caroline Hales

Contact Phone Number: 043855999 ext .......

Project explained by:

Principal Investigator

Signature:

Principal Investigator

Date:

Version 2

Page 2

$27 / 06 / 2014$ 


\section{Appendix 6: Patient study information sheet}

\section{Patient Information Sheet}

\begin{tabular}{|l|l|}
\hline Principal Investigator: & Contact Details: \\
\hline Caz Hales & \\
PhD student / researcher & \\
Registered Nurse & \\
& \\
\hline
\end{tabular}

TITLE:

How do nurses care for very overweight patients in the intensive care setting?

\section{INTRODUCTION:}

You are invited to take part in a study which looks at how the nurses within Intensive Care Unit care for you during your hospital admission in the unit. If you would like to take part in the study, I will discuss the details with you within the next day or two. You are under no obligation to take part and as such your care will not differ from that of other participants.

\section{ABOUT THE STUDY}

The aim of the study is to examine the everyday activities, social interactions and influences within the intensive care setting in which nurses' care for larger patients.

All patients who are admitted to Intensive Care Unit who have a BMI $\geq 40$ $\mathrm{kg} / \mathrm{m} 2$ (obesity score based on height and weight), receiving non-weight loss care and is expected to remain in the unit for more than twelve hours have been considered for the study. It is expected that there will be about four to eight patients and approximately all the nursing staff involved in the study.

The study will take place within the Intensive Care Unit a

hospital over a three to four month period. However, your participation will be for the duration of your intensive care admission. Once you have been discharged from Intensive Care Unit, either to the ward or an alternative hospital your participation in the study will be complete. Should you be readmitted to Intensive Care Unit within the study period then consent will be requested from you to continue with study participation.

During the study, I will be observing the nurses as they care for you throughout your intensive care admission and writing notes on what I see. The focus of the observation will be on how the nursing team interact with you, other nurses and health professionals to provide appropriate levels of care to support your recovery. Additionally, I will be focussing on what resources are used and what resources are needed, if not available, to provide care that is 
supportive of your individual needs. At no point during the study will conversations overheard during periods of observation be recorded and used as part of the data collection.

To provide an understanding of your needs and how the nurses have interpreted and planned your care I will be requesting to read your medical notes and daily charts. The information required from your medical notes will be your demographics (age, ethnicity, gender etc), past medical history and current health issues, reasons for admission to the unit, initial treatment plan and any limitations of treatment regimes/modalities.

All information taken from your notes will be written in note form and coded so that you remain unidentifiable. Any identifying information will not be included in the notes taken. All observation notes and medical information will be coded and stored in a secure and locked environment.

As part of the study, the nurses who care for you will be invited to be interviewed so that they can provide further insight and understanding into how they care for you on a daily basis. These interviews will be audio-taped, transcribed and coded so that any information specific to you will be unidentifiable. Should an identifiable detail be discussed during the interview then this will be removed from the data collection. Again, the data collected from the interviews will coded and all audiotapes and written notes will be stored in a secure and locked environment.

After the study has been completed and the results have been published the data collected will be stored for ten years in a secure and locked environment and then destroyed.

\section{BENEFITS RISKS AND SAFETY}

There are no direct or immediate benefits of taking part in the study as the study is based on the observation of your care and as such is non-therapeutic. However, your participation and the knowledge gained from observing your care will hopefully benefit future patients with morbid obesity who are admitted to the intensive care setting. There are no perceived risks to taking part in the study as your care does not differ from that of non-participants.

The inclusion and exclusion criteria for the study are: Patient Inclusion

1. $\mathrm{A}$ BMI $\geq 40 \mathrm{~kg} / \mathrm{m} 2$

2. Admission to ICU for reasons other than bariatric (weight loss) surgery

3. Is expected to remain in ICU for more than twelve hours

4. Consents to study participation

Patient Exclusion

1. Admitted to ICU following elective bariatric (weight loss) surgery

2. Is expected to be discharged from ICU within twelve hours

3. Does not consent to study participation

Taking part in the study will not cost you or your family / whanau anything. Additionally, the researcher is not in a position to provide payment or incentives to participants for their participation in the study.

\section{PARTICIPATION}

Your participation is entirely voluntary (your choice). You do not have to take part in this study, and if you choose not to take part this will not affect any future care or treatment. 
If you do agree to take part you are free to withdraw from the study at any time, without having to give a reason and this will in no way affect your continuing health care.

\section{GENERAL}

Should you require further information about the study this can be provided by myself or Professor Jo Ann Walton (Research supervisor). Contact details: jo.walton@vuw.ac.nz Ph: 04 4636135.

If you need an interpreter, one can be provided for you by the hospital throughout the duration of your hospital stay. Please let me know if you would like me to organise an interpreter for you.

Should you wish to have a friend, family or whanau support to help you understand the risks and/or benefits of this study and any other explanation you may require then please let me know so that I can ensure that they are present during all of our conversations about the study.

If you have any questions or concerns about your rights as a participant in this research study you can contact an independent health and disability advocate. This is a free service provided under the Health and Disability Commissioner Act.

Telephone: (NZ wide) 0800555050

Free Fax (NZ wide): 080027877678 (0800 2 SUPPORT)

Email (NZ wide): advocacy@hdc.org.nz"

Alternatively, you can contact the Central Regional Ethics committee who have approved the study on:

Telephone: (04) 4962405

Email: central ethicscommittee@moh.govt.nz

\section{CONFIDENTIALITY}

No material which could personally identify you will be used in any reports on this study.

Persons identified as having access to confidential information are:

1. Caz Hales- Principal researcher

2. Professor Jo Ann Walton- Researcher supervisor (Head of Graduate School, Nursing, Midwifery and Health)

3. Confidential typist if needed to transcribe data

\section{RESULTS}

A significant delay may occur between data collection and publication of the results due to the nature of the research. However, participants can receive information regarding the outcomes of the study should they wish by contacting myself. The results of the study will be published within an appropriate medical/ nursing journal and submitted as part of a $\mathrm{PhD}$ thesis to Victoria University of Wellington.

\section{STATEMENT OF APPROVAL}

This study has received ethical approval from the Central Regional Ethics Committee.

Please feel free to contact the researcher if you have any questions about this study. 


\section{Patient Consent Form}

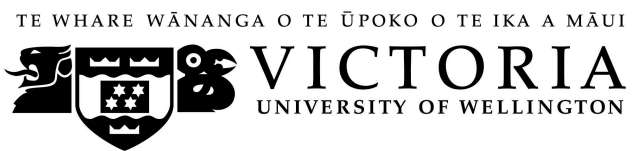

Title: What is the culture of care for very overweight patients in the intensive care setting?

Principal Investigator: Caroline Hales

Participant's Name

\section{REQUEST FOR INTERPRETER}

\begin{tabular}{|l|l|c|c|}
\hline English & I wish to have an interpreter. & Yes & No \\
\hline Maori & $\begin{array}{l}\text { E hiahia ana ahau ki tetahi kaiwhakamaori/kaiwhaka pakeha } \\
\text { korero. }\end{array}$ & Ae & Kao \\
\hline $\begin{array}{l}\text { Cook } \\
\text { Island }\end{array}$ & Ka inangaro au i tetai tangata uri reo. & Ae & Kare \\
\hline Fijian & Au gadreva me dua e vakadewa vosa vei au & Io & Sega \\
\hline Niuean & Fia manako au ke fakaaoga e taha tagata fakahokohoko kupu. & E & Nakai \\
\hline Samoan & Ou te mana'o ia i ai se fa'amatala upu. & Ioe & Leai \\
\hline Tokelaun & $\begin{array}{l}\text { Ko au e fofou ki he tino ke fakaliliu te gagana Peletania ki na } \\
\text { gagana o na motu o te Pahefika }\end{array}$ & Ioe & Leai \\
\hline Tongan & Oku ou fiema'u ha fakatonulea. & Io & Ikai \\
\hline
\end{tabular}

I have read and I understand the information sheet dated for volunteers taking part in the study designed to observe and interview nurses caring for very overweight patients within the intensive care unit. I have had the opportunity to discuss this study. I am satisfied with the answers I have been given.

I have had the opportunity to use whanau support or a friend to help me ask questions and understand the study.

I understand that taking part in this study is voluntary (my choice) and that I may withdraw from the study at any time and this will in no way affect my continuing health care.

I have had this project explained to me by

I understand that my participation in this study is confidential and that no material which could identify me will be used in any reports on this study. 
I have had time to consider whether to take part.

I wish to receive a copy of the results.

I have been advised that a significant delay may occur between data collection and publication of the results.

I would like the researcher to discuss the outcomes of the study with me.

YES/NO

I (full name) hereby consent to take part in this study.

Date:

Signature:

Participant

Researcher: Caroline Hales

Contact Phone Number: 043855999 ext .......

Project explained by: Principal Investigator

Signature: Principal Investigator

Date: 


\section{Appendix 8: Interview lengths}

\begin{tabular}{|c|c|c|c|c|c|}
\hline Participant & $\begin{array}{l}\text { Interview } \\
\text { length }\end{array}$ & Participant & $\begin{array}{l}\text { Interview } \\
\text { length }\end{array}$ & Participant & $\begin{array}{l}\text { Interview } \\
\text { length }\end{array}$ \\
\hline \multicolumn{2}{|c|}{ Staff nurses } & \multicolumn{2}{|c|}{ Staff nurses } & \multicolumn{2}{|c|}{ Doctors } \\
\hline Caroline & $38 \mathrm{mins} 22 \mathrm{sec}$ & Rachel & $22 \mathrm{mins} 18 \mathrm{sec}$ & Alexis & $25 \mathrm{mins} 10 \mathrm{sec}$ \\
\hline Cathyrn & $29 \mathrm{mins} 51 \mathrm{sec}$ & Rita & $28 \mathrm{mins} 49 \mathrm{sec}$ & David & $40 \mathrm{mins} 22 \mathrm{sec}$ \\
\hline Ella & $43 \mathrm{mins} 33 \mathrm{sec}$ & Robyn & $36 \mathrm{mins} 36 \mathrm{sec}$ & James & $55 \mathrm{mins} 32 \mathrm{sec}$ \\
\hline Helen & 29mins 49sec & Roma & $26 \mathrm{mins} 30 \mathrm{sec}$ & John & 39mins 19sec \\
\hline Jackie & $32 \mathrm{mins} 08 \mathrm{sec}$ & Rose & $26 \mathrm{mins} 03 \mathrm{sec}$ & Julian & $37 \mathrm{mins} 43 \mathrm{sec}$ \\
\hline Jenny & $21 \mathrm{mins} 12 \mathrm{sec}$ & Ruth & $25 \mathrm{mins} 26 \mathrm{sec}$ & \multicolumn{2}{|c|}{ Senior nurses } \\
\hline Joanne & $51 \mathrm{mins} 28 \mathrm{sec}$ & Sally & $22 \mathrm{mins} 13 \mathrm{sec}$ & Beryl & $30 \mathrm{mins} 22 \mathrm{sec}$ \\
\hline Kate & $34 \mathrm{mins} 37 \mathrm{sec}$ & Sandy & $36 \mathrm{mins} 11 \mathrm{sec}$ & Bob & $42 \mathrm{mins} 28 \mathrm{sec}$ \\
\hline Laura & $23 \mathrm{mins} 24 \mathrm{sec}$ & Shirley & $41 \mathrm{mins} 30 \mathrm{sec}$ & Florence & $43 \mathrm{mins} 35 \mathrm{sec}$ \\
\hline Lee & $51 \mathrm{mins} 31 \mathrm{sec}$ & Sophie & $35 \mathrm{mins} 29 \mathrm{sec}$ & George & $57 \mathrm{mins} 03 \mathrm{sec}$ \\
\hline Lucy & 34mins $44 \mathrm{sec}$ & Stella & 37 mins $06 \mathrm{sec}$ & Glenda & $49 \mathrm{mins} 20 \mathrm{sec}$ \\
\hline Maggie & $26 \mathrm{mins} 11 \mathrm{sec}$ & Sue & $17 \mathrm{mins} 47 \mathrm{sec}$ & Jane & $35 \mathrm{mins} 42 \mathrm{sec}$ \\
\hline Mary Anne & $26 \mathrm{mins} 34 \mathrm{sec}$ & Trudy & $24 \mathrm{mins} 07 \mathrm{sec}$ & Milly & $15 \mathrm{mins} 43 \mathrm{sec}$ \\
\hline Max & $35 \mathrm{mins} 46 \mathrm{sec}$ & Vicki & $34 \mathrm{mins} 10 \mathrm{sec}$ & Phillippe & $38 \mathrm{mins} 46 \mathrm{sec}$ \\
\hline Molly & $25 \mathrm{mins} 31 \mathrm{sec}$ & Yvonne & $40 \mathrm{mins} 10 \mathrm{sec}$ & Rebecca & $56 \mathrm{mins} 28 \mathrm{sec}$ \\
\hline
\end{tabular}


Appendix 9: Semi-structured interview guide

\begin{tabular}{|l|l|}
\hline Interview No. Name: & Date: \\
\hline Demographics & \\
1. How many years in nursing do you have? & \\
2. How many years have you worked in ICU? & \\
3. What ethnicity do you identify yourself with? &
\end{tabular}

\section{Experience}

1. Can you describe what it is like to care for an obese patient?

2. Can you recount an example of caring for an obese patient that you will never forget?

3. How does caring for an obese patient differ from non-obese patients? What's different?

4. Can you describe the nursing challenges of caring for an obese patient?

5. How does caring for an obese patient here differ with other places that you have worked?

6. How do you personally feel about caring for obese patients?

- does it differ?

- what would be the reasons for this?

7. Do you see or touch obese patients differently?

8. A potential $300 \mathrm{~kg}$ patient was going to be admitted before Christmas from another hospital and I'm interested to know what your initial thoughts would be around hearing that there was a patient of $300 \mathrm{kgs}$ going to be admitted to the unit?

- What planning would you be thinking about?

9. I'm really interested in the language that people use when they're describing obese patients. So what words would you use to describe an obese patient and in what context? Clinical bedside/ handover/non-clinical/socially. What words have you heard your colleagues use and in what context?

\section{Observation clarity}

1. I've noticed that nurses feel really uncomfortable acknowledging or mentioning in front of the patient during handover that they are obese? Why do you think this is? How do you feel about discussing obesity in front of the patient?

2. During shift handover, the ACMN's rarely mention the patient is obese- why do you think this might be?

- What importance do you put on information when deciding what information to handover over?

3. For my study I'm using a BMI 40 which clinically classifies someone as being morbidly obese or bariatric. Some of these patients are weighing around 115 to $120 \mathrm{kgs}$,-some of the nursing staff have asked why are they in the study. When do nurses/doctors perceive somebody to be obese, when would you decide that a patient's obese what sort of things would you take into consideration?

4. I've noticed that obese patients appear physically very different. Are you able to describe the appearances of obese patients and how this might affect care?

\section{Attitudes}

1. What is your personal opinion about why people are obese?

2. Have you ever considered yourself to be obese?

- Do you think this affects the way you think and care for obese patients?

3. Is anyone in your family obese and has their experiences influenced your opinions?

- Do you think your family upbringing has influenced your perceptions of obesity? Why?

4. A statement: 'Obesity has been described as the last socially accepted form of prejudice in society.' What do you think about this statement and why?

\section{Resources}

1. What resources do you have available to care for obese patients?

2. When would you make the decision to use the bariatric room?

3. Have you ever received any specialist bariatric training or education? 


\section{Appendix 10: Data management spreadsheet}

\section{Data management audit trail}

\begin{tabular}{|c|c|c|c|c|c|c|}
\hline & Transcript accuracy & Add annotations & Upload NVivo & Ist code & Profile memo & 2nd code \\
\hline \multicolumn{7}{|l|}{ Doctors } \\
\hline Alexis & 6/09/11 & 6/09/11 & 6/09/11 & 6/09/11 & 6/09/11 & $8 / 11 / 11$ \\
\hline David & $14 / 06 / 11$ & $14 / 06 / 11$ & $23 / 06 / 11$ & $28 / 06 / 11$ & $28 / 06 / 11$ & $8 / 11 / 11$ \\
\hline James & $14 / 06 / 11$ & $14 / 06 / 11$ & $23 / 06 / 11$ & $28 / 06 / 11$ & $28 / 06 / 11$ & $8 / 11 / 11$ \\
\hline John & $13 / 06 / 11$ & $13 / 06 / 11$ & $23 / 06 / 11$ & 7/07/11 & 7/07/11 & $9 / 11 / 11$ \\
\hline Julian & $13 / 06 / 11$ & $13 / 06 / 11$ & $23 / 06 / 11$ & 7/07/11 & 7/07/11 & $9 / 11 / 11$ \\
\hline \multicolumn{7}{|c|}{ Senior nurses } \\
\hline Beryl & $6 / 09 / 11$ & $6 / 09 / 11$ & $6 / 09 / 11$ & $27 / 06 / 11$ & $27 / 06 / 11$ & $9 / 11 / 11$ \\
\hline Bob & $14 / 06 / 11$ & $14 / 06 / 11$ & $23 / 06 / 11$ & $27 / 06 / 11$ & $27 / 06 / 11$ & $9 / 11 / 11$ \\
\hline Florence & $15 / 06 / 11$ & $15 / 06 / 11$ & $23 / 06 / 11$ & $29 / 06 / 11$ & $29 / 06 / 11$ & $10 / 11 / 11$ \\
\hline George & $14 / 06 / 11$ & $14 / 06 / 11$ & $23 / 06 / 11$ & $30 / 06 / 11$ & $30 / 06 / 11$ & $10 / 11 / 11$ \\
\hline Glenda & $15 / 06 / 11$ & $15 / 06 / 11$ & $23 / 06 / 11$ & $30 / 06 / 11$ & $30 / 06 / 11$ & $10 / 11 / 11$ \\
\hline Jane & $16 / 06 / 11$ & $16 / 06 / 11$ & $23 / 06 / 11$ & 6/07/11 & 6/07/11 & $11 / 11 / 11$ \\
\hline Milly & $22 / 06 / 11$ & $22 / 06 / 11$ & $23 / 06 / 11$ & $3 / 08 / 11$ & $3 / 08 / 11$ & $11 / 11 / 11$ \\
\hline Phillippe & $17 / 06 / 11$ & $17 / 06 / 11$ & $23 / 06 / 11$ & $3 / 08 / 11$ & $3 / 08 / 11$ & $11 / 11 / 11$ \\
\hline Rebecca & $20 / 06 / 11$ & $20 / 06 / 11$ & $23 / 06 / 11$ & 5/08/11 & 5/08/11 & $11 / 11 / 11$ \\
\hline \multicolumn{7}{|l|}{ Staff nurses } \\
\hline Caroline & $15 / 06 / 11$ & $15 / 06 / 11$ & $23 / 06 / 11$ & $27 / 06 / 11$ & $27 / 06 / 11$ & $9 / 11 / 11$ \\
\hline Cathyrn & $15 / 06 / 11$ & $15 / 06 / 11$ & $23 / 06 / 11$ & $27 / 06 / 11$ & $27 / 06 / 11$ & $9 / 11 / 11$ \\
\hline Ella & $15 / 06 / 11$ & $15 / 06 / 11$ & $23 / 06 / 11$ & $28 / 06 / 11$ & $28 / 06 / 11$ & $9 / 11 / 11$ \\
\hline Helen & $16 / 06 / 11$ & $16 / 06 / 11$ & $23 / 06 / 11$ & $1 / 07 / 11$ & $1 / 07 / 11$ & $14 / 11 / 11$ \\
\hline Jackie & $16 / 06 / 11$ & $16 / 06 / 11$ & $23 / 06 / 11$ & $4 / 07 / 11$ & $4 / 07 / 11$ & $14 / 11 / 11$ \\
\hline Jenny & $6 / 09 / 11$ & $6 / 09 / 11$ & $6 / 09 / 11$ & $5 / 07 / 11$ & $5 / 07 / 11$ & $15 / 01 / 11$ \\
\hline Joanne & $16 / 06 / 11$ & $16 / 06 / 11$ & $23 / 06 / 11$ & 7/07/11 & $7 / 07 / 11$ & $15 / 11 / 11$ \\
\hline Kate & $17 / 06 / 11$ & $17 / 06 / 11$ & $23 / 06 / 11$ & 8/07/11 & 8/07/11 & $16 / 11 / 11$ \\
\hline Laura & $17 / 06 / 11$ & $17 / 06 / 11$ & $23 / 06 / 11$ & 8/07/11 & 8/07/11 & $16 / 11 / 11$ \\
\hline Lee & $15 / 06 / 11$ & $15 / 06 / 11$ & $23 / 06 / 11$ & $15 / 07 / 11$ & $15 / 07 / 11$ & $17 / 11 / 11$ \\
\hline Lucy & $17 / 06 / 11$ & $17 / 06 / 11$ & $23 / 06 / 11$ & $15 / 07 / 11$ & $15 / 07 / 11$ & $18 / 11 / 11$ \\
\hline Maggie & $17 / 06 / 11$ & $17 / 06 / 11$ & $23 / 06 / 11$ & $18 / 07 / 11$ & $18 / 07 / 11$ & $18 / 11 / 11$ \\
\hline Mary Anne & $22 / 06 / 11$ & $22 / 06 / 11$ & $23 / 06 / 11$ & $25 / 07 / 11$ & $25 / 07 / 11$ & $19 / 11 / 11$ \\
\hline Max & $20 / 06 / 11$ & $20 / 06 / 11$ & $23 / 06 / 11$ & $1 / 08 / 11$ & $1 / 08 / 11$ & $21 / 11 / 11$ \\
\hline Molly & $20 / 06 / 11$ & $20 / 06 / 11$ & $23 / 06 / 11$ & $3 / 08 / 11$ & $3 / 08 / 11$ & $21 / 11 / 11$ \\
\hline Rachel & $20 / 06 / 11$ & 20/06/11 & $23 / 06 / 11$ & $5 / 08 / 11$ & $5 / 08 / 11$ & $21 / 11 / 11$ \\
\hline Rita & $21 / 06 / 11$ & $21 / 06 / 11$ & $23 / 06 / 11$ & $8 / 08 / 11$ & $8 / 08 / 11$ & $21 / 11 / 11$ \\
\hline Robyn & $21 / 06 / 11$ & $21 / 06 / 11$ & $23 / 06 / 11$ & $8 / 08 / 11$ & $8 / 08 / 11$ & $22 / 11 / 11$ \\
\hline Roma & $21 / 06 / 11$ & $21 / 06 / 11$ & $23 / 06 / 11$ & 9/08/11 & 9/08/11 & $22 / 11 / 11$ \\
\hline Rose & $21 / 06 / 11$ & $21 / 06 / 11$ & $23 / 06 / 11$ & $22 / 08 / 11$ & $22 / 08 / 11$ & $23 / 11 / 11$ \\
\hline Ruth & $21 / 06 / 11$ & $21 / 06 / 11$ & $23 / 06 / 11$ & $22 / 08 / 11$ & $22 / 08 / 11$ & $24 / 11 / 11$ \\
\hline Sally & $17 / 06 / 11$ & $17 / 06 / 11$ & $23 / 06 / 11$ & $23 / 08 / 11$ & $23 / 08 / 11$ & $25 / 11 / 11$ \\
\hline Sandy & $21 / 06 / 11$ & $21 / 06 / 11$ & $23 / 06 / 11$ & $23 / 08 / 11$ & $23 / 08 / 11$ & $25 / 11 / 11$ \\
\hline Shirley & $21 / 06 / 11$ & $21 / 06 / 11$ & $23 / 06 / 11$ & $23 / 08 / 11$ & $23 / 08 / 11$ & $25 / 11 / 11$ \\
\hline Sophie & $22 / 06 / 11$ & $22 / 06 / 11$ & $23 / 06 / 11$ & $26 / 08 / 11$ & $26 / 08 / 11$ & $25 / 11 / 11$ \\
\hline Stella & $22 / 06 / 11$ & $22 / 06 / 11$ & $23 / 06 / 11$ & $26 / 08 / 11$ & $26 / 08 / 11$ & $28 / 11 / 11$ \\
\hline Sue & $22 / 06 / 11$ & $22 / 06 / 11$ & $23 / 06 / 11$ & $26 / 08 / 11$ & $26 / 08 / 11$ & $29 / 11 / 11$ \\
\hline Trudy & $21 / 06 / 11$ & $21 / 06 / 11$ & $23 / 06 / 11$ & $29 / 08 / 11$ & $29 / 08 / 11$ & $29 / 11 / 11$ \\
\hline Vicki & $22 / 06 / 11$ & $22 / 06 / 11$ & $23 / 06 / 11$ & $31 / 08 / 11$ & $31 / 08 / 11$ & $30 / 11 / 11$ \\
\hline Yvonne & $22 / 06 / 11$ & $22 / 06 / 11$ & $23 / 06 / 11$ & 6/09/11 & 6/09/11 & $30 / 11 / 11$ \\
\hline
\end{tabular}


Data management audit trail

\begin{tabular}{|l|l|l|l|l|l|}
\hline Patients f/notes & Transcribed E/C & Upload NVivo & Ist code & Profile memo & 2nd code \\
\hline Patient A & $6 / 06 / 11$ & $25 / 06 / 11$ & $2 / 10 / 11$ & $7 / 11 / 11$ & $2 / 12 / 11$ \\
\hline Patient B & $7 / 06 / 11$ & $25 / 06 / 11$ & $2 / 10 / 11$ & $7 / 11 / 11$ & $2 / 12 / 11$ \\
\hline Patient C & $7 / 06 / 11$ & $25 / 06 / 11$ & $2 / 10 / 11$ & $7 / 11 / 11$ & $2 / 12 / 11$ \\
\hline Patient D & $9 / 06 / 11$ & $25 / 06 / 11$ & $4 / 10 / 11$ & $7 / 11 / 11$ & $2 / 12 / 11$ \\
\hline Patient E & $9 / 06 / 11$ & $25 / 06 / 11$ & $4 / 10 / 11$ & $7 / 11 / 11$ & $2 / 12 / 11$ \\
\hline Patient F & $10 / 06 / 11$ & $25 / 06 / 11$ & $5 / 10 / 11$ & $7 / 11 / 11$ & $5 / 12 / 11$ \\
\hline Patient G & $15 / 06 / 11$ & $25 / 06 / 11$ & $5 / 10 / 11$ & $7 / 11 / 11$ & $5 / 12 / 11$ \\
\hline
\end{tabular}

\begin{tabular}{|l|l|l|l|}
\hline Patients med notes & Upload Nvivo & 1st Code & 2nd Code \\
\hline Patient A & $10 / 10 / 11$ & $10 / 10 / 11$ & $9 / 12 / 11$ \\
\hline Patient B & $10 / 10 / 11$ & $10 / 10 / 11$ & $9 / 12 / 11$ \\
\hline Patient C & $10 / 10 / 11$ & $10 / 10 / 11$ & $9 / 12 / 11$ \\
\hline Patient D & $10 / 10 / 11$ & $10 / 10 / 11$ & $9 / 12 / 11$ \\
\hline Patient E & $10 / 10 / 11$ & $12 / 10 / 11$ & $9 / 12 / 11$ \\
\hline Patient F & $10 / 10 / 11$ & $12 / 10 / 11$ & $9 / 12 / 11$ \\
\hline Patient G & $10 / 10 / 11$ & $12 / 10 / 11$ & $9 / 12 / 11$ \\
\hline
\end{tabular}

\begin{tabular}{|l|l|l|l|}
\hline Policies & Upload Nvivo & 1st code & 2nd code \\
\hline Manual handling & $10 / 10 / 11$ & $13 / 10 / 11$ & $16 / 12 / 11$ \\
\hline Bariatric transport & $10 / 10 / 11$ & $13 / 10 / 11$ & $16 / 12 / 11$ \\
\hline ICU orientation & $10 / 10 / 11$ & $13 / 10 / 11$ & $16 / 12 / 11$ \\
\hline Care of Bariatric pts & $10 / 10 / 11$ & $13 / 10 / 11$ & $16 / 12 / 11$ \\
\hline
\end{tabular}

\begin{tabular}{|l|l|l|l|l|l|}
\hline \multicolumn{5}{|c|}{ Thematic analysis } & \\
\hline Raw data coded & \multicolumn{1}{|c|}{ As above } & & & & \\
\hline Topic codes-recoding & $19 / 12 / 11$ & $20 / 12 / 11$ & $21 / 12 / 11$ & & \\
\hline Coded data collapsed (1) & $11 / 09 / 12$ & $12 / 09 / 12$ & $26 / 09 / 12$ & & \\
\hline Coded data collapsed (2) & $3 / 12 / 12$ & $4 / 12 / 12$ & $5 / 12 / 12$ & $6 / 12 / 12$ & $7 / 12 / 12$ \\
\hline
\end{tabular}


Appendix 11: Data analysis: Example of operational definitions for raw data codes

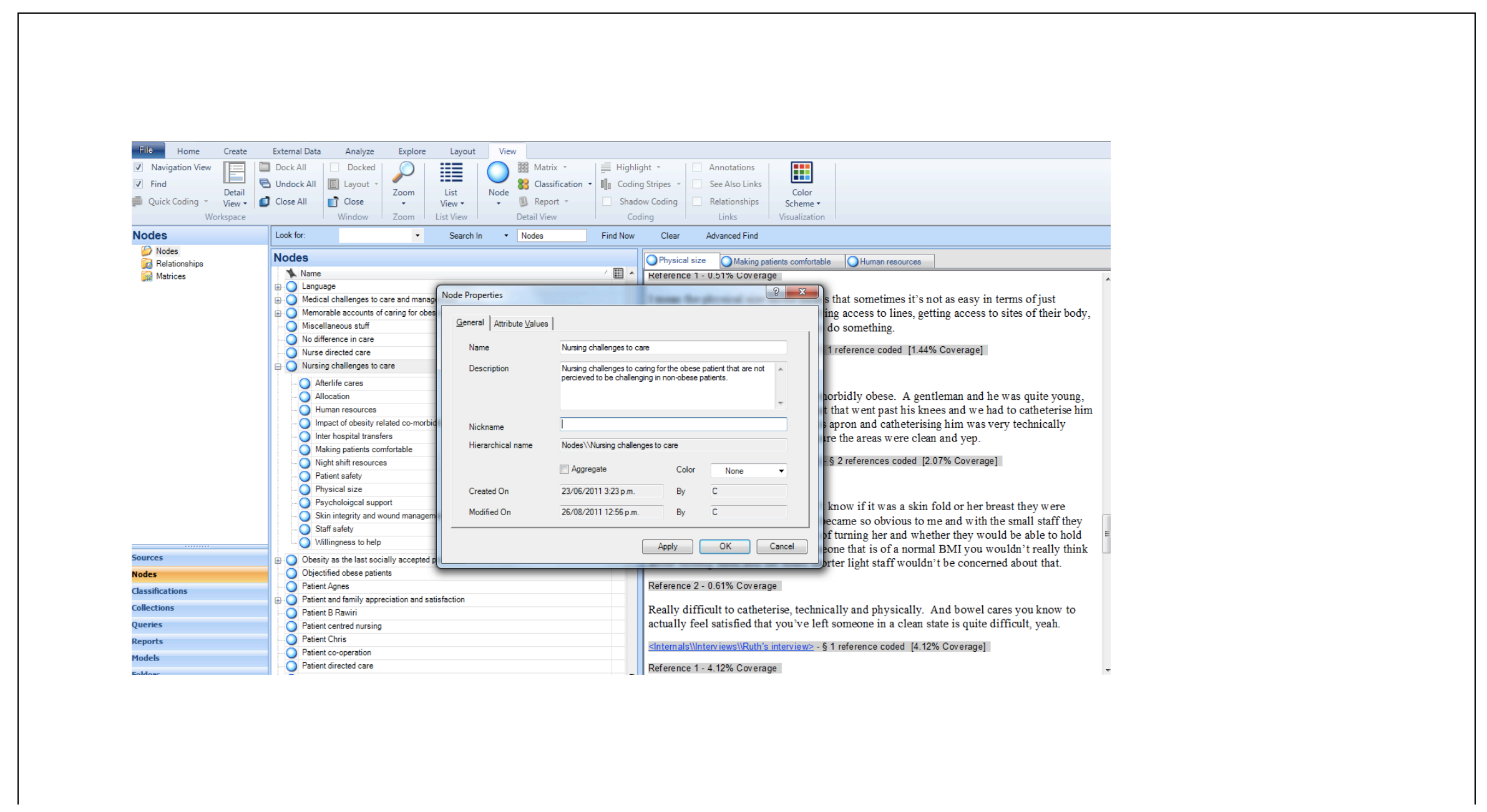


Appendix 12: Data analysis: Example of raw data coding

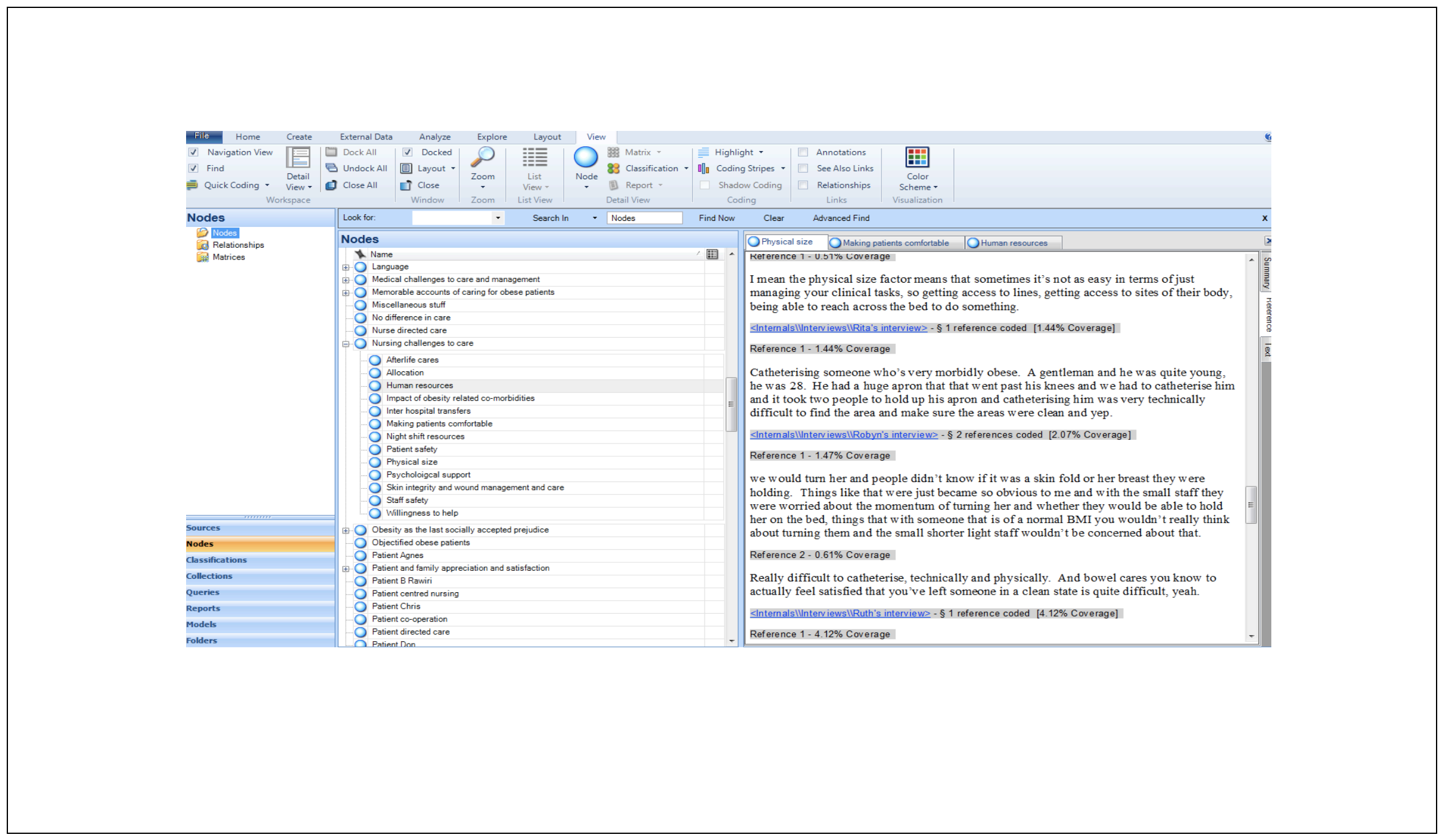




\section{Appendix 13: Data analysis/ conceptualisation}

PhD Analysis diagram: Misfits

\begin{tabular}{|c|c|c|c|}
\hline First order or open coding (Raw data codes/ categories) & $\begin{array}{l}\text { Second order or axial cod } \\
\text { /Concepts) }\end{array}$ & gg (Themes & $\begin{array}{l}\text { Confirmation or selective } \\
\text { coding }\end{array}$ \\
\hline 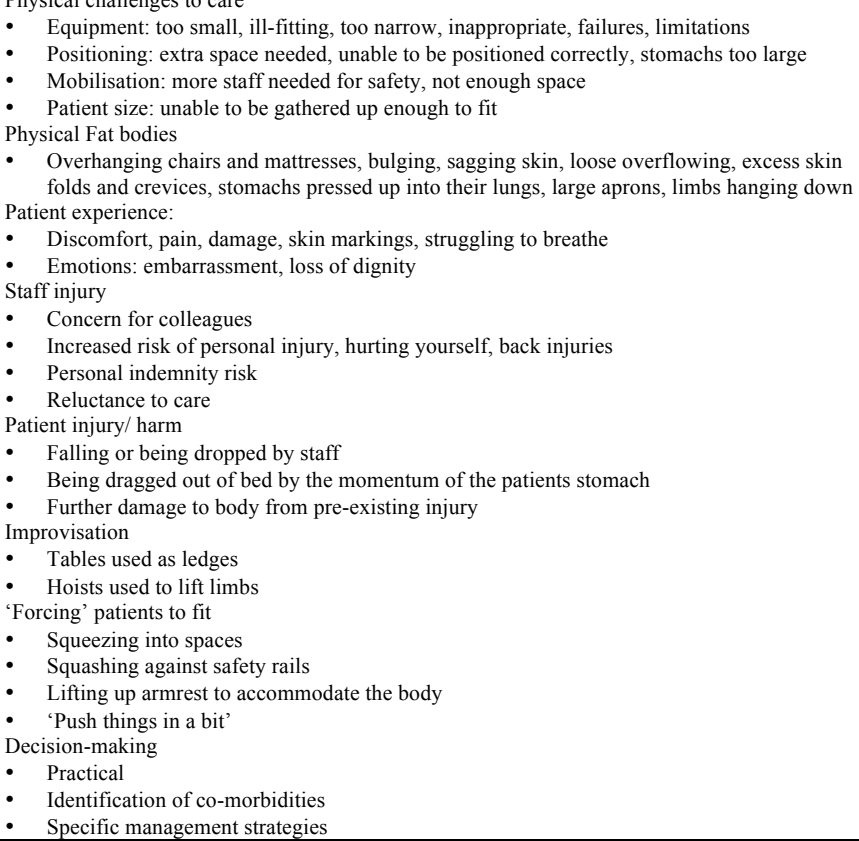 & $\begin{array}{l}\text { Do not fit physically into } \\
\text { the space of the ICU } \\
\text { Not fitting in physically }\end{array}$ & $\begin{array}{l}\text { Misfits in ICU } \\
\text { Front stage story of } \\
\text { care } \\
\text { What was observed } \\
\text { by others }\end{array}$ & $\begin{array}{l}\text { Managing the mis-fitted fat } \\
\text { body through professional and } \\
\text { private face activities } \\
\text { Seeping/merging of backstage } \\
\text { and front stage } \\
\text { (Behavioural regions) } \\
\text { (Face-work) } \\
\text { (Emotional labour) }\end{array}$ \\
\hline
\end{tabular}


PhD Analysis diagram: Misfits

\begin{tabular}{|c|c|c|c|}
\hline $\begin{array}{l}\text { Medical challenges: } \\
\text { Altered physiology, } \\
\text { Increased technicality, } \\
\text { Increased co-morbidities, } \\
\text { Difficult to assessment, missed diagnose, } \\
\text { Increased of complications, } \\
\text { Nursing chace manaenges: } \\
\text { - } \text { Inable to perform effective CPR, } \\
\quad \text { Increased intervention technicalities ie catheterisation }\end{array}$ & $\begin{array}{l}\text { Medically do not fit } \\
\text { normal ways to practice } \\
\text { Issues of anatomy, } \\
\text { physiology, and } \\
\text { intervention techniques } \\
\text { 'Fat as risk' }\end{array}$ & & \\
\hline $\begin{array}{l}\text { Staff attitudes } \\
\text { Strive for normal weight } \\
\text { - } \\
\text { Bat patient attributes } \\
\text {. Feelings about why patients are fat/ ability to lose weight/ cause of obesity epidemic } \\
\text { Financial burden } \\
\text { Taking responsibility for own health (normative expectations) } \\
\text { Moral dialogue } \\
\text { - Personal failings } \\
\text { Derogative physical descriptions }\end{array}$ & $\begin{array}{l}\text { Socially do not fit } \\
\text { Prejudice } \\
\text { 'Them and us' } \\
\text { Moral judging } \\
\text { Contempt }\end{array}$ & $\begin{array}{l}\text { Non-professional } \\
\text { behaviour } \\
\text { Private feelings } \\
\text { Private expressions } \\
\text { amongst other staff } \\
\begin{array}{l}\text { Back stage story of } \\
\text { care }\end{array}\end{array}$ & \\
\hline $\begin{array}{l}\text { Social vulnerability } \\
\text { Awkwardness } \\
\text { - Social awkwardness (behaviour) } \\
\circ \quad \text { Avoidance: } 0 \text { ot acknowledging or mentioning a patients obesity/ pretense } \\
\circ \quad \text { Secret codes } \\
\text { - } \\
\text { Emotional awkwardness (feelings) } \\
\circ \quad \text { Concealing emotions } \\
\circ \quad \text { Fencing emotions } \\
\text { Language usage: } \\
\text { Bedside language } \\
-\quad \text { Staff room language }\end{array}$ & $\begin{array}{l}\text { Responding to the } \\
\text { stigma } \\
\text { Conscious masking of } \\
\text { private feelings }\end{array}$ & $\begin{array}{l}\text { Managing feelings } \\
\text { Conscious behavior } \\
\text { modifications during } \\
\text { patient care } \\
\text { Act or performance } \\
\text { Professional } \\
\text { behaviour }\end{array}$ & \\
\hline
\end{tabular}


